RICE UNIVERSITY

\title{
Modeling of Intense Laser Driven High Energy Density Plasmas
}

by

\author{
Matthew Chase Levy
}

A Thesis Submitted

in Partial Fulfillment of the

Requirements For the Degree

Doctor of Philosophy

Approved, Thesis Committee:

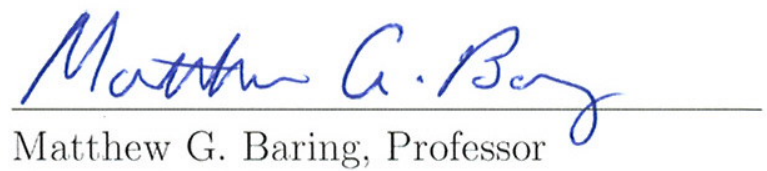

Physics \& Astronomy

Thesis Co-advisor

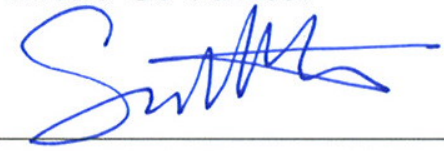

Scott C. Wilks, Staff Physicist

Lawrence Livermore National Laboratory

Thesis Co-advisor

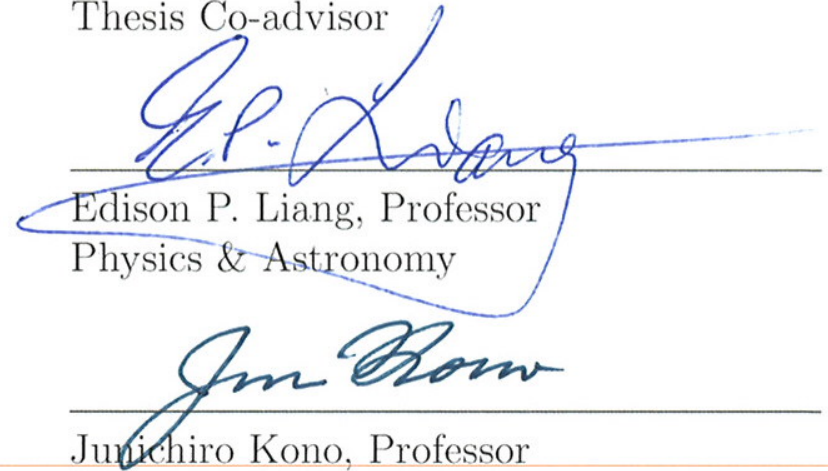

Electrical \& Computer Engineering

Houston, Texas

October, 2014 


\section{Abstract}

Illuminating matter with petawatt (one quadrillion watts) laser light creates extreme states of plasmas with temperatures exceeding ten million degrees Celsius and pressures exceeding one billion earth atmospheres. These high energy density conditions are driven at the microscopic scale by dense currents of relativistic electrons, oscillating violently in the intense laser fields, as well as the plasma processes arising when these particles lose phase coherence and are injected into bulk target material. Suitably harnessed, this setup opens the way to compact relativistic particle accelerators, laser fusion energy sources, laboratory astrophysics, ultrafast imaging systems, proton cancer therapies, anti-matter creation, high-energy radiation sources and intense high harmonic generation. In this thesis, theoretical models of the absorption of high power laser light by matter are derived, applications of these models are investigated, and simulation tools supporting the diagnosis and implementation of these applications are developed.

In particular, an advanced, relativistically-correct theoretical model of petawatt laser absorption by optically-thick targets is developed, accounting for both ion and electron beam aspects of the interaction. Predictions of the model for the energetic properties of these beams, as well as the dynamical motion of the laser-matter interface, are elucidated. Results from high resolution, relativistic, kinetic particle-in-cell simulations using the LSP code are shown to be in good agreement with the model. The theoretical maximum and minimum absorption values in laser-solid interactions are derived from the model in a general fashion, constraining nonlinear absorption

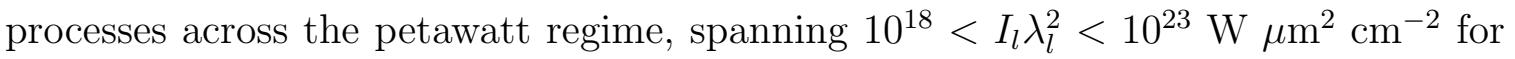
intensity $I_{l}$ and wavelength $\lambda_{l}$. These results are shown to bound several dozens of published experimental and simulation data points, underlining the usefulness of the 
model. These results are extended to include effects related to heterogeneous plasmas, including relativistically-underdense plasmas relevant to 'pre-plasma' situations, and realistic laser spatio-temporal profiles. Our dynamic considerations of absorption processes are then extended to the 10-petawatt scale. In a manner that could support reaching the QED-plasma regime, a mechanism of focusing high power laser light to higher intensities is elucidated. Supporting the measurement and validation of these models, the development of a new simulation tool for understanding high energy density plasmas, based on the proton radiography technique, is detailed. 


\section{Acknowledgements}

Over the past $5^{1 / 2}$ years it has often seemed that pursuit of graduate studies has formed an exercise in the stewardship of resources entrusted to me by advisors, mentors, collaborators and others. In endeavors bearing fruit, my stewardship of these resources has seemed adequate. In the totality of outcomes, to these people I wish to express my warmest and deepest gratitude.

I first thank my advisors Scott Wilks and Matthew Baring, and close collaborators Max Tabak and Steve Libby. Working with this group has entailed diligent work, but has been so pleasurable, thrilling and intellectually exhilarating. I am sincerely grateful to Steve for sharing much of his wisdom and personality with me, and for his early encouragement of my research into basic relativistic laser-plasma systems. To Max, for sharing your tremendous creativity and intellectual rigor, and for teaching me the arts of vigorous scientific debate, thank you. I further wish to thank Matthew for his continuous support, perspective and insight over the years. Finally, I will be forever grateful to Scott, from whom I have learned more than I am able to express, and who is a man as humble and as brilliant as any I have ever met.

I am deeply grateful to Edward Moses for his mentorship over the years, and to him, Bill Goldstein and Don Correll for recruiting me to Livermore. I thank Andreas Kemp for acting as my mentor for two summers in my first years of graduate school, and for teaching me the essentials of kinetic simulations and relativistic laser theory. I am grateful also to Laurent Divol for his encouragement and for stimulating discussions. For their most essential support of endeavors at Stanford and Oxford, to

Siegfried Glenzer, Andrea Schmidt, Chris Barty, Chris Keane, Peter Norreys, Gianluca Gregori and Don I offer my warmest gratitude.

It has been a great pleasure to work together with Dmitri Ryutov, Hye-Sook Park 
and my colleagues in the ACSEL collaboration. Meeting every week for the past three years has been both challenging and exciting as we have uncovered and deciphered new aspects in the emerging field of laboratory astrophysics. I wish to especially thank our core group of Steve Ross, Channing Huntington, Nathan Kugland, Frederico Fiuza, Steve Weber and Bruce Remington. Thanks are also due to Anatoly Spitkovsky, Youichi Sakawa, Rich Petrasso and Chikang Li for many stimulating conversations.

At Livermore I am particularly grateful to Tony Link for interesting and useful discussions on intense laser-plasma interactions and on the LSP code. I also wish to thank my colleagues Elijah Kemp, Fred Perez, Prav Patel, Brad Pollock, Dave Strozzi, Alex Friedman, Art Park, Eddie Dewald, Tammy Ma, Joe Ralph, Drew Higginson, Bill Meyer, Bruce Cohen, Andy Mackinnon, John Moody and Erik Storm. Thanks are also in order to Wayne Meir and Harry McLean for parsing countless review and release documents.

I am grateful to Troy Carter for giving me my start in research plasma physics in his lab at STRB. For stimulating conversations and support over the years I thank also Edison Liang, Tom Killian, Malcolm Haines, Douglass Schumacher, Wojciech Rozmus, Alex Arefiev, Boris Breizman, Manuel Hegelich, John Wheater and Bob Bingham.

For their support and companionship through my graduate studies I would like to thank my friends from Los Angeles, Tokyo, San Francisco and elsewhere around the world. To Martin, Misha, Colleen, James, Paul, Trent, Masaki, Nick, Claudio, Eric, Anton, Andrey, Colleen, Mario, Emil, Jeff, and many others, thank you. Finally, this work would have been impossible without the enduring support of my family: my parents Lawrence and Joanie, my brother Andrew, my step mother Lori, as well as my aunts and uncles John Rubin, Emily Rubin and Julie Nameth.

My research has been supported by the Lawrence Scholarship at the Lawrence 
Livermore National Laboratory, for which I offer my sincere gratitude to John Knezovich, Christine Zachow, Anna Wright, Susan Lowder, Shelley Rose, Elaine Johnson and Lynnette Gomez. This work was performed under the auspices of the U.S. Department of Energy by Lawrence Livermore National Laboratory under Contract DE-AC52-07NA27344. 
Dedicated to my father, Lawrence J. Levy. 


\section{Contents}

List of Figures vi vi v v v

1 Introduction 1

1.1 Applications of intense laser driven high energy density plasmas . . . 1

1.1.1 Inertial confinement fusion . . . . . . . . . . . . . . 1

1.1.2 Fast ignition using high power lasers . . . . . . . . . . 5

1.1.3 Compact relativistic particle accelerators . . . . . . . . . . . 11

1.1.4 Ultrafast imaging systems . . . . . . . . . . . . . 15

1.1.5 Laboratory astrophysics . . . . . . . . . . . . . . . 17

1.1.6 Probing the quantum vacuum structure . . . . . . . . . . 23

1.2 Theory of intense laser-plasma interactions . . . . . . . . . . 26

1.2.1 Particle dynamics in ultraintense light fields . . . . . . . . . 26

1.2.2 Acceleration of high density plasma due to the steady-state ponderomotive force . . . . . . . . . . . . . . 32

1.2.3 Relativistic electron beam generation . . . . . . . . . . . . 35

1.2.4 Quantum electrodynamical effects at ultra-relativistic intensity 38

1.3 Thesis structure overview . . . . . . . . . . . . . . . . 42

\section{Description of particle-in-cell simulation methods 43}

2.1 Description of the particle-in-cell scheme . . . . . . . . . . . . . 44

2.2 Details of selected numerical schemes implemented in the LSP code . 52 


\section{Absorption of ultraintense laser light by solid matter 56}

3.1 Conservation laws and conversion efficiency in ultraintense laser-overdense plasma interactions . . . . . . . . . . . . 56

3.1.1 Coupling of ultraintense laser light to an overdense plasma . . 57

3.1.2 Relation between the piston and hole punching velocities . . . 61

3.1.3 Poynting theorem for ultraintense light . . . . . . . . . . . . . 64

3.1.4 Fully-relativistic model of ultraintense laser light absorption . 67

3.1.5 Conversion efficiency into hot electrons and hole punching ions 74

3.1.6 Particle-in-cell simulations and possible astrophysical

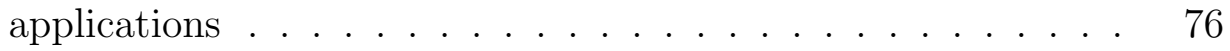

3.2 Hot electron divergence in the kinematic analysis of relativistic light on solids . . . . . . . . . . . . . . . . . . . . 84

3.3 Simple causal model of electron angular divergence in ultraintense laser-plasma interactions . . . . . . . . . . . . . . . . . 90

3.4 Relativistic plane wave model of electron beam generation . . . . . . 95

3.5 Petawatt laser absorption bounded . . . . . . . . . . . . . . 100

3.5.1 Relativistic interaction model . . . . . . . . . . . . . . . 103

3.5.2 Absorption bounds .................. 105

3.5.3 Comparison between absorption bounds and published data . 107

3.5.4 Absorption bounds in terms of laser and plasma parameters . 109

3.6 Phase space accessible to the petawatt laser-plasma system . . . . . . 110

\section{Relativistic laser interactions with realistic heterogeneous} plasmas

4.1 Accelerating piston action and plasma heating in high energy density laser-plasma interactions . . . . . . . . . . . . . . . 115 
4.1.1 Development of a self-consistent framework for equations of motion ........................ 117

4.1.2 Analytical derivation of the front velocity for a realistic pulse envelope in the ponderomotive regime . . . . . . . . . . . . 120

4.1.3 Consideration of simple closure models for the effective electron temperature . . . . . . . . . . . . . . . . . 123

4.1.4 Comparisons of the analytical model to results from PIC simulations . . . . . . . . . . . . . . . . . . 128

4.1.5 Qualitative modifications to ion phase space . . . . . . . . 130

4.2 Predictive model for ultraintense laser preplasma using ponderomotive-driven ions . . . . . . . . . . . . . . . . . . 133

4.2.1 Diagnosing preplasma conditions using the reflected laser light 136

4.2.2 Diagnosing preplasma conditions using the time-integrated ion energy spectrum . . . . . . . . . . . . . . . . . . . 142

4.2.3 Application to measuring laser parameters in high-contrast situations ........................ 144

\section{Focusing of intense subpicosecond laser pulses in wedge} targets

5.0.4 Numerical modeling of laser focusing in wedge targets . . . . . 150

5.0.5 Characterizations of dynamical pulse-target evolution . . . . . 153

\section{Development of interpretative proton radiography simu-} lation tool for HED plasmas $\quad 166$

6.1 Features of PRIME f . . . . . . . . . . . . . . . . . . . . 168

6.1.1 Tools for constructing electromagnetic fields . . . . . . . . . . 170 
6.1.2 Specifying source and detector properties . . . . . . . . . . . . 172

6.2 Benchmarking against analytic theory . . . . . . . . . . . . 173

6.3 Application to the filamentation instability in millimeter-scale HED plasmas . . . . . . . . . . . . . . . . . . . . 180

7 Conclusions \& future research 195

$\begin{array}{ll}\text { References } & 198\end{array}$ 


\section{List of Figures}

1.1 Schematic showing the steps involved in the successful implementation of the indirect drive approach to inertial confinement fusion $(\mathrm{ICF})[2] \ldots \ldots \ldots \ldots \ldots \ldots \ldots$

1.2 The upper image shows a schematic of the hohlraum and fusion capsule suspended at its center. The bottom image shows this same setup, realized in cryogenic ICF experiments at the National Ignition Facility $(\mathrm{NIF})[148,2] \ldots \ldots \ldots \ldots \ldots$

1.3 Schematic of a fast ignition ICF hohlraum[2]. In this design a gold cone is used to guide the ultrafast ignitor pulse and shield its path from coronal plasma generated during the fuel compression stage of the process. . . . . . . . . . . . . . . .

1.4 Comparison between fast ignition and the conventional indirect drive ICF approaches. The left plot illustration the differences in density and temperature space[2]. The right plot indicates the ideal fusion gain curves $G$ for both the fast ignition (isochoric) and conventional (isobaric) approaches, with the lower axis corresponding to the laser energy driving the fuel compression.[204]. . . . . . . . . . . . . . . 
1.5 Overview of the physics involved in the coupling of the ignitor pulse energy to the imploding DT fuel. The upper image shows a schematic which is realized in the lower experimental image.[57] In the latter image the transparent shell attached to the cone tip acts as a surrogate for the ignition DT fuel. . . . . . . . . . . . . . . . . . 10

1.6 Phase space (a) and energy distribution (b) of ions accelerated by the target normal sheath acceleration mechanism (TNSA), from ref. [216]. The peak ion energy in (b) reaches $4 \mathrm{MeV} . \ldots . . . . .$.

1.7 Experimental spectra of ions accelerated by the TSNA and BOA mechanisms. The curves showing exponential-like spectrum are associated with the former process and quasi-monoenergetic spectra with the latter process, from ref. [74] . . . . . . . . . . . . . . 14

1.8 Experimental proton radiography results showing megagauss-scale electromagnetic fields in inertial confinement fusion experiments, from ref. [115]. The spatial scale of each image is $2.9 \mathrm{~mm} \times 2.9 \mathrm{~mm}$. Panels show a time series of images captured at 0.85, 1.6, 2.17 and $2.8 \mathrm{~ns}$, from left to right. . . . . . . . . . . . . . . 15

1.9 Experimental proton radiography image of plasma filmamentation resulting from growth of the electromagnetic Weibel instability[209] in laboratory astrophysics experiments, from ref. [77] (details and experimental conditions found therein). . . . . . . . . . . . 18 
1.10 Large-scale self-organization of electromagnetic fields in laboratory astrophysics experiments, from ref. [97]. The upper panel shows a schematic of the experiments in which high energy lasers are used to ablate two collinear plastic targets. The ablated plasma flows expand at supersonically, colliding with one another. The lower panel shows a series of experimental proton radiography image of self-organizing electromagnetic field structures emerging from this situation. . . . . .

1.11 Progression of intense laser technology over time, adapted from ref. [212]. The chirped-pulse amplification (CPA) technique[203] is seen to be responsible for enabling the rapid growth of high intensity laser systems, extending into the petawatt regime. . . . . . . . . .

1.12 Depictions of key features of petawatt laser-plasma interactions from two dimensional particle-in-cell simulations. $z_{1}$ corresponds to the current axial position of the laser-matter interface and $z_{2}$ corresponds to the target rear; scales have been omitted to emphasize the generality of the features across typical conditions. (a) shows a configuration space map of the plasma electron density during the period of laser irradiation. The electric field magnitude in the region containing the laser is shown in (b). The color scale in this plot progresses from blue to green to red as the field strength increases. (c) and (d) depict the electron and ion phase space in the simulation

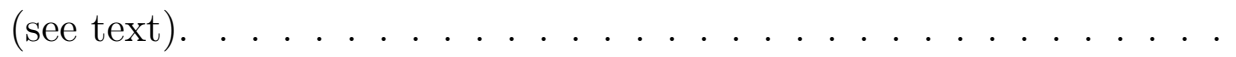

1.13 Contours of $u_{p} / c$, the rate at which a petawatt laser punches into a high density target, as a function of the normalized laser intensity and electron plasma density. . . . . . . . . . . . . . . 33

1.14 Threshold for the onset of relativistic ion acceleration effects (see text). 35 
1.15 Comparison between models of the effective temperature of the relativistic electron beam (see text). . . . . . . . . . .

1.16 Effects of radiative friction on relativistic electron motion in the plasma skin depth as a function of laser dimensionless field strength $a_{0}$. That the cooling length $\ell_{c}$ exceeds the skin depth $\ell_{s}$ while $a_{0}<300$ confirms that QED effects must be taken into account for 10-petawatt scale laser-plasma interactions. . . . . . . . . . . . . . . . 40

1.17 Threshold for the onset of QED effects in petawatt laser-solid interactions as a function of laser dimensionless field strength $a_{0}$ for a variety of plasma densities, using equations (1.23) and (1.24). . . . . 41

2.1 Schematic showing the use of macroparticles in the PIC scheme to represent groups of identical particles, from ref. [166]. Collectively the macroparticles fully sample the phase space distribution of the system.

2.2 Schematic illustrating the 'leapfrog' time stepping sequence associated with the explicit solution method, from ref. [210]. This figure shows that the Vlasov equation governing the particle phase space evolution $(x, p)$ and Maxwell's equations governing the electromagnetic field evolution $E, B$ are advanced in an alternating fashion. . . . . . . . . 50

2.3 Schematic illustrating the predictive time stepping sequence associated with the direct implicit solution method. This figure shows that due to the predictive nature of the algorithm particles are effectively advanced twice. . . . . . . . . . . . . . . . . 53 
3.1 Schematic depicting the laser plasma coupling in the interaction region with $n_{0}=n_{e}=Z n_{i}$ where $\mathrm{Z}$ is the ion charge state. The laser piston boundary is represented by the dashed line. . . . . . . . . . 58

3.2 Contours of the $O(1)$ term comprising $\gamma_{h}$ as given by equation (3.38). $\quad 74$

3.3 Analytic solutions satisfying energy and momentum conservation with the laser. (a) depicts the energetic electron Lorentz factor $\gamma_{h}$ and (b) shows the piston velocity $\beta_{p}$. (c-d) show contours of the laser conversion efficiencies into ions and into energetic electrons, respectively. 75

3.4 Comparison to particle-in-cell simulations. (a-d) Light coupling into the steady-state and oscillatory ponderomotive absorption modes. (e) Piston velocity $\beta_{p}$ from the simulations and analytic model. Solid lines correspond to $d x_{p} / d t$ and diamond markers correspond to the velocity inferred from $1 \omega$ doppler shift measurements from the simulations (see text). Dashed lines represent the analytic $\beta_{p}$ from equation (3.37). . . . . . . . . . . . . . . .

3.5 Simulation and analytics for the $\beta_{0}=0.3$ laser-plasma interaction. (a-b) Ion and (d-e) electron phase space from simulations. (c) Hot electron spectra from simulations (relative $n(\mathcal{E})$ not to scale). (f) Comparison of laser absorption analytic predictions and simulation

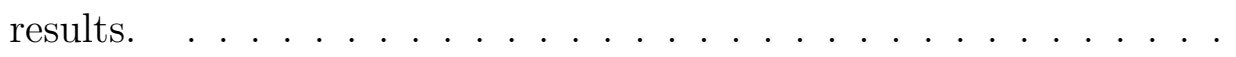


3.6 Coupling to the oscillatory component of the laser ponderomotive force for the $\beta_{0}=0.3, \Delta \phi=0.92$ simulation. (a) Number density of exchange-mediating electrons (red) and of ions (black). Arrows indicate the position of the laser-plasma interface. (b) Hot electron density $n_{h}$ and $\bar{\rho}_{h}$. (c) Comparison of hot electron absorption from the simulation to the analytic model. (d) Ensemble average Lorentz factors (see text). . . . . . . . . . . . . . .

3.7 Relative changes to solutions as a function of the hot electron beam divergence angle $\theta$. Quantities are normalized to their $\theta=0$ values. . 88

3.8 Absolute changes in energy partitioning as a function of $\theta$. . . . . . 88

3.9 Schematic depicting the evolution of the plasma critical surface over time for an ultraintense laser pulse having Gaussian transverse spatial profile. Electron generation is also depicted. In (C), we see the unit vector normal to the critical surface as a function of radial position and time, $\hat{x_{f}}(\theta, t)$. The directionality $\hat{\phi}$ and magnitude $P_{e} / P_{L}$ of the hot electrons relative to $\hat{x_{f}}$ at each spatial point is a function of laser

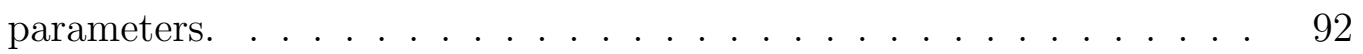

3.10 Models of hot electron beam temperature including relativistic plane wave calculations (see text). . . . . . . . . . . . 
3.11 Schematic showing key features of the petawatt laser-solid interaction. A high-power laser with strength parameter $a_{0}>1$ is shown striking an overdense target, interacting over the Lorentz-transformed collisionless skin depth $\ell_{\mathrm{s}}$ (dark green region), and exciting a highly-relativistic electron flux (red spheres) and moderately-relativistic ion flux (blue spheres). Laser and excited particle properties are connected across $\ell_{\mathrm{S}}$ by applying relativistic Rankine-Hugoniot-like relations at the laser-matter interface, allowing abstraction of downstream effects, e.g., scattering in the $x>\ell_{\mathrm{s}}$ target (light green region). Depiction uses a frame of reference co-moving with the interface. . . . . . . . . . . . . . . . . . 102

3.12 Bounds on absorption and sub-partitioning of absorbed light. The lower-right axis corresponds to $\beta_{0}$ describing the initial laser and target conditions, and the total absorption $f=1-\mathcal{R}$. Two surfaces corresponding to the absorption into electrons $f_{\mathrm{e}}$ and into ions $f_{\mathrm{i}}$ are shown (the former having slight transparency for visualization purposes). Contours of the optimization function $\gamma_{\mathrm{e}}$ are superimposed on these surfaces using dark gray. The lower limit $f_{*}$ (blue) and upper limit $f^{*}$ (red) on absorption are shown bounding $f_{\mathrm{e}}$ and $f_{\mathrm{i}}$. . 106 
3.13 Comparison between absorption bounds and published data. The complete dataset compiled in Davies[37] is reproduced here, spanning experimental and simulation data published over the past two decades, across a variety of laser and plasma conditions. Dashed lines corresponding to fits of selected experimental data are shown to guide the eye. Additional high-intensity simulation data is reproduced from Levy et al.[106] The upper limit on absorption $f^{*}$ is depicted in red and the lower limit $f_{*}$ in blue, with forbidden regions indicated using shading. The two outlying data points correspond to simulations of (†) a very thin $0.2 \mu \mathrm{m}$ pre-deformed target, and ${ }^{(\dagger)}$ an essentially underdense $n_{\mathrm{c}}>n_{0}$ interaction, both violating assumptions underpinning the laser-solid model. . . . . . . . . . . . . . . . . . 108

3.14 Fraction of phase space accessible to the petawatt laser-plasma system, $\Lambda$ (see text). . . . . . . . . . . . . . . 112

3.15 Rate of change of the $\Lambda$ with $\beta_{0}$ indicating that the number of states accessible to the laser-plasma system is maximized for $\beta_{0} \sim 0.1$ (see text). Note different scales relative to Fig. 3.14. . . . . . . . . . . . . 113

4.1 Schematic depicting the temporal evolution of the front velocity $u_{f}$ (red) for Gaussian temporal laser intensity $I(t, x)$ (dashed orange) incident on an exponential plasma density profile. Shading below the $u_{f}$ curve represents the doppler shifted light reflected from the critical interface. Initially the plasma expands with $u_{f}<0$ at a rate determined by bulk heating associated with laser prepulse. $u_{f}$ is then seen to evolve through three distinct dynamical regimes over time, denoted by I, II and III (see text). . . . . . . . . . . . . . . . . . 121 
4.2 Front velocity and hole boring depth dependence on laser intensity and pulse temporal profiles in the $\Gamma(t) \gg 1$ regime. Intensities depicted are $I_{19}$ (blue), $I_{20}$ (purple) and $I_{21}$ (green), with dashed and solid lines representing semi-infinite and Gaussian pulse envelopes respectively. (A) Front velocity $u_{f} / c$; the dashed orange line depicts the temporal pulse profile. (B) Front location $x_{f}$. System parameters are $\ell=15 \mu m, x_{s}=30 \mu m, t_{1 / 2}=1.4 p s . \ldots \ldots$

4.3 Dependence of the characteristic expansion velocity scale $u_{t h}$ on laser intensity and $t_{s}$. (A) $u_{t h}$ for a single laser intensity $I_{20}$ for different values of $t_{s}$. (B) $u_{t h}\left(t_{s}=3 p s\right)$ for three different laser intensities, $I_{19}$ (blue), $I_{20}$ (purple), $I_{21}$ (green). The dashed orange line depicts the temporal laser pulse profile, with system parameters $\ell=10 \mu m, x_{s}=30 \mu m, t_{1 / 2}=1.4 p s . \ldots \ldots \ldots$

4.4 (A) Comparison of Gaussian temporal pulse PIC simulation results with the analytical model for $\Delta \lambda / \lambda_{0}$, with regimes (I-III) labeled below. (B-C) Ion phase space density from the simulations at 2,3ps in units of $\log n / n_{c r}$, with results from the Liouville code overlaid in red.127

4.5 (A) Comparison of semi-infinite pulse PIC simulation results with the analytical model for $\Delta \lambda / \lambda_{0}$, with regimes (I-III) labeled below. (B-C) Ion phase space density from the simulations at 2,3ps in units of $\log n / n_{c r}$, with results from the Liouville code overlaid in red (note different transverse scales). . . . . . . . . . . . . . . . . . 129 
4.6 Schematic depicting the analytic front velocity $u_{p}$ curves for the semi-infinite (purple) and Gaussian temporal (blue) pulse shapes, through equation (4.13) and (4.14) respectively. For both shapes, the peak laser intensity is $4 \times 10^{20} \mathrm{~W} / \mathrm{cm}^{2}$ and the plasma conditions are $\ell_{g}=30 \mu \mathrm{m}, x_{s}=100 \mu \mathrm{m}, n_{0} / n_{c r}=100$ and $M_{i} / m_{p}=2$. The Gaussian pulse peaks at $1.5 \mathrm{ps}$ with a full-width half-maximum of $0.5 \mathrm{ps} . \quad$. . . 138

4.7 Front velocity dependence on pre-plasma scalelength for a Gaussian temporal laser pulse. Plasma and laser conditions are the same as in Fig. 4.6, with the scalelength varying from $\ell_{g}=30 \mu \mathrm{m}$ (blue), $15 \mu \mathrm{m}$ (purple) and $3 \mu \mathrm{m}$ (green). The curve is seen to peak sharply at early time for steep density profiles. . . . . . . . . . . . . . . . . . 139

4.8 Application of the analytical model for $u_{p}$ to existing experimental data[162]. The thin dashed-red line represents the normalized laser intensity profile. The solid colored curves represent pre-plasma scalelength, varying in $1 \mu \mathrm{m}$ intervals from $\ell_{g}=1 \mu \mathrm{m}$ (innermost blue) to $6 \mu \mathrm{m}$ (outermost purple), with the outlying dark yellow curve representing $30 \mu \mathrm{m}$ for comparison. The black data points and error bars of \pm 0.012 in $\Delta \lambda / \lambda_{0}$ correspond to the experimental data (see text). . . . . . . . . . . . . . . . . . 141

4.9 Time-integrated target-rear ion spectra for the parameters used in Naumova et al.[150]. (A) shows the simulation results along with their analytic model (red curve) for a semi-infinite pulse envelope. (B) shows the distribution from our Liouville numerical code for the same conditions. (C) shows the Liouville results for the same parameters, using a Gaussian temporal pulse (see text). . . . . . . . . 143 
4.10 Analytic $u_{p}$ curves from equation (4.16) in the high-contrast pulse limit. Starting from the inner-most blue curve of $I_{0}=1.3 \times 10^{20}$ $\mathrm{W} / \mathrm{cm}^{2}$ and moving outward, each colored curve represents an increase in laser intensity by a factor of two, such that the outer-most purple curve has $I / I_{0}=10$. . . . . . . . . . . . . . . . . . . 145

5.1 Schematic for the $\theta_{17}$ target configuration with the low intensity $a=0.1$ reference beam (see text); laser longitudinal Poynting flux $P_{z}$ shows $I_{\text {peak }} / I_{0}=10.8$ peak focusing with superimposed white dashed lines indicating initial target position. . . . . . . . . . . . . . . 152

5.2 Laser peak intensity dependence on target angle for the $a_{3}$ pulse (see

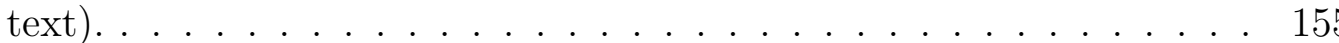

5.3 (A) Spatial location of peak laser intensity for the $\theta_{17}$ target; front velocity $u_{f}$ and time evolution of $z_{\text {peak }}$ (see text). (B) Scaling of $u_{f}$ with dimensionless laser parameter $a$ in $\theta_{17}$. The analytic model from equation (5.8) and its asymptotic form are represented by the solid and dashed blue curves respectively. . . . . . . . . . . . . . . . 156

5.4 Temporal evolution of the $a_{3} \theta_{17}$ pulse-target configuration in $Y-Z$ space. (A-C) Time-averaged electron density $\log n_{e} / n_{c r}$ with superimposed white contour lines corresponding to densities of $0.1 n_{c r}$ and $n_{c r} ;(\mathrm{D}-\mathrm{F})$ time-averaged laser Poynting flux $\log P_{L}$ $\left[W / \mathrm{cm}^{2}\right] \ldots \ldots \ldots \ldots \ldots \ldots$

5.5 Laser energy absorption into target walls at 300 fs for the $a_{3}$ laser pulse (note different transverse scales). . . . . . . . . . . . . . 162 
5.6 Hot electron characteristics at 1.4 ps across target geometries for the $a_{3}$ laser pulse; energy flux densities of forward-going electrons $\log P_{e}$ $\left[W / \mathrm{cm}^{2}\right]$ (note different transverse scales). . . . . . . . . . . . 163

6.1 Schematic of key aspects of the proton radiography simulation tool, following the propagation of protons along the $z$ axis from left to right. Parameters controlling the proton source are described in section 6.1.2. As an example, specifying the source control vector $\vec{S}=\left(2,14.7 \mathrm{MeV}, 10^{9}, 1 \mathrm{~cm}\right)$ creates a $14.7 \mathrm{MeV}$ monoenergetic proton source isotropically emitting 1 billion protons, imitating a realistic D ${ }^{3}$ He source, situated $\left|z_{s}\right|=1 \mathrm{~cm}$ from the object plane containing the plasma electromagnetic fields. Depicted in the object plane at $z=0$ are four tilted ellipsoidal magnetic filaments, each having form given by equation (6.3). These fields are created in PRIME by specifying the single field control vector $\vec{L}=(4,2,2,100 \mu \mathrm{m}, 100 \mu \mathrm{m}, 50 \mu \mathrm{m}, 500 \mu \mathrm{m}, 0,0,1 \mathrm{MG})$, as described in section 6.1.1. A simulated proton radiograph created by the tool is shown in the image plane, situated at $z=z_{i}$. Details on the field structure underpinning this radiograph are covered in section 6.3. . . 167 
6.2 Comparison between theory and simulation results in the linear regime. The situations shown here interact a monoenergetic $\mathcal{E}_{p}=14.7$ $\mathrm{MeV}$ proton source of $\left|z_{s}\right|=1 \mathrm{~cm}, z_{i}=10 \mathrm{~cm}$ with a single magnetic filament of the form given by equation (6.3) having $a=100 \mu \mathrm{m}$ and $b=300 \mu \mathrm{m}$. (a-b) show simulated radiograph results using $B_{0}=0.2 \times B_{0 c r i t}$ for the focusing and defocusing cases, respectively, while (d-e) use $B_{0}=0.9 \times B_{0 \text { crit }}$. The color scale is fixed between images with darker (lighter) regions indicating a surplus (deficit) of protons. (c) and (f) depict normalized lineouts of the proton fluence along $y_{0}=0$ for the $0.2 \times B_{0 c r i t}$ and $0.9 \times B_{0 c r i t}$ simulations, respectively. The blue curves correspond to the focusing cases and the yellow curves to the defocusing cases. The simulations agree with the theory predictions of equations (6.13-6.14), indicated using dashed black curves, to better than $5 \%$ in all cases. . . . . . . . . . 174 
6.3 Comparison between theory and simulation results in the caustic regime. In these simulations a monoenergetic $\mathcal{E}_{p}=14.7 \mathrm{MeV}$ proton source of $\left|z_{s}\right|=1 \mathrm{~cm}, z_{i}=10 \mathrm{~cm}$ interacts with a single magnetic filament of the form given by equation (6.3) having $a=100 \mu \mathrm{m}$ and $b=300 \mu \mathrm{m}$. (a-b) show simulated radiograph results using $B_{0}=2 \times B_{0 \text { crit }}$ for the focusing and defocusing cases, respectively. The color scale is fixed between images with darker (lighter) regions indicating a surplus (deficit) of protons. (c) shows the multi-branched caustic structures predicted by the parametric equations (6.14) and (6.15) using $\epsilon=0.5$. (d) shows normalized lineouts of the simulated proton fluence along $y_{0}=0$ with the blue curve corresponding to the focusing case and the yellow curve corresponding to the defocusing case. The complete analytic results formed by summing over all three branches of each curve in (c) are indicated by the dashed black lines in (d), exhibiting close agreement with the simulation results. . . . . 178

\subsection{Comparison of simulation fields to the relevant transverse Weibel} instability modes. The black curves correspond to the normalized instability growth rates $\Gamma(k)$ for collisionless Carbon (dotted), collisional Carbon (dashed) and collisional $\mathrm{CH}_{2}$ flows (solid) from equations (6.16-6.17). The colored curves correspond to normalized Fourier transformations $\log B_{x}(k)$ across $\hat{x}$ at the simulation midplane for three simulations of 'forests' of magnetic filaments (see text). . . 181 
6.5 Effects of field strength $B_{0}$ and probing proton energy $\mathcal{E}_{p}$ on the simulated proton radiograph results. All panels here correspond to the sim. 99 configuration indicated by the green curve in Fig. 6.4. (a-b) correspond to probing proton energies of $\mathcal{E}_{p}=14.7 \mathrm{MeV}$ and (c-d) to $\mathcal{E}_{p}=3 \mathrm{MeV}$. (a) and (c) correspond to $B_{0}=1 M G$ (meaning a peak simulation field of $0.4 M G$ ), and (b) and (d) correspond to $B_{0}=0.3 M G \ldots \ldots \ldots \ldots \ldots$

6.6 Synthetic proton radiographs for (a) sim. 18 and (b) sim. 45. Across simulations $B_{0}=1 M G$ and $\mathcal{E}_{p}=14.7 \mathrm{MeV}$. The radiograph corresponding to these conditions for sim. 99 is shown in Fig. 6.5 (a).

6.7 Comparison between field periodicity and proton radiograph image periodicity. Solid lines correspond to Fourier-transformed lineouts at $y_{0}=0$ of the synthetic radiographs shown in Fig. 6.6. The dashed curves in (b-d) correspond to $2 \pi / k$ for the simulation $k$-vectors shown in Fig. 6.4. . . . . . . . . . . . . . . . . . . . . . . 186

6.8 Role of filament tilting and of the simulation field $k$-vector function on the resulting proton radiograph signal. (a-b) show synthetic radiographs generated by $P R I M E$ using the field control vector $\vec{L}=(4,16,16,200 \mu \mathrm{m}, 200 \mu \mathrm{m}, 50 \mu \mathrm{m}, 500 \mu \mathrm{m}, 0,0,0.3 \mathrm{MG})$, and the same proton source and detector as in Figs. 6.4 - 6.7. (a) corresponds to the application of individualized tilt parameters $\delta \psi, \delta \theta=15^{\circ}$ while (b) uses $\delta \psi, \delta \theta=0 \ldots \ldots \ldots$. . . . . . . . . . 187

6.9 Fourier transformations of the normalized lineouts of proton fluence along $y_{0}=0$ for the radiographs depicted in Fig. 6.8. The purple curve corresponds to the $\delta \psi, \delta \theta=15^{\circ}$ case while enhanced signal in the midplane is absent for the $0^{\circ}$ case. . . . . . . . . . . . . . 189 
6.10 Proton radiographs of the the sim. 99 field geometry imaged along the axis of the plasma flows. The $14.7 \mathrm{MeV}$ proton source is otherwise identical to that used for Figs. 6.5 - 6.8. (a) corresponds to $B_{0}=0.3 \mathrm{MG}$ and (b) corresponds to $B_{0}=1 \mathrm{MG}$. . . . . . . . . . 190

6.11 Proton radiography imaging along the plasma flow axis. Apart from the proton axis of propagation the simulation field and proton source properties are identical to those covered in Fig. 6.8. (a) corresponds to $\delta \psi, \delta \theta=0$ while (b) uses $\delta \psi, \delta \theta=15^{\circ}$. . . . . . . . . 192 


\section{Chapter 1}

\section{Introduction}

Intense laser interactions with matter can heat and compress millimeter-scale volumes to extreme temperatures, densities and pressures[34, 169, 43]. In these high energy density (HED) interactions the quiver momentum of free electrons oscillating in the light fields becomes relativistic: $p_{e} /\left(m_{e} c\right)>1$ for electron momentum $p_{e}$, mass $m_{e}$ and speed of light $c$ when $I_{1} \lambda_{1}^{2}>10^{18} \mathrm{~W} \mu \mathrm{m}^{2} \mathrm{~cm}^{-2}$ for laser intensity $I_{1}$ and wavelength $\lambda_{\mathrm{l}}$. Above the relativistic threshold laser light is primarily absorbed collisionlessly and the particle dynamics are strongly kinetic. This thesis documents a five year theoretical

exploration of aspects of the rich physical processes operating in this regime. It begins with a discussion of the potential high-impact societal, scientific and medical applications which are enabled by detailed understanding of these processes.

\subsection{Applications of intense laser driven high energy density plasmas}

\subsubsection{Inertial confinement fusion}

Inertial confinement fusion (ICF) is a high energy laser-based approach to creating self-sustaining thermonuclear fusion plasmas in the laboratory $[154,179,124]$. ICF seeks to produce an 'igniting' or 'burning' plasma by using laser energy to compress and heat a millimeter-scale Deuterium-Tritium (DT) capsule to high densities and temperatures such that the two nuclei overcome the Coulomb barrier and fuse. Each 


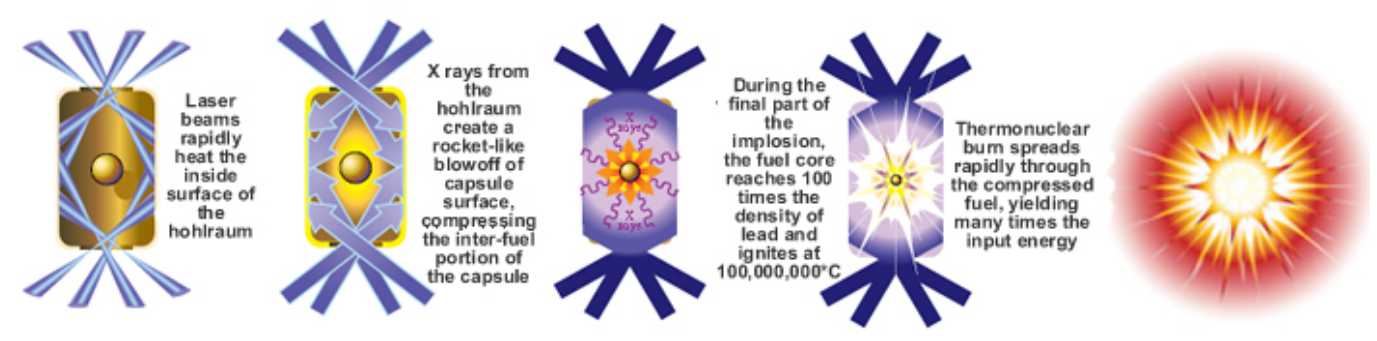

Figure 1.1 : Schematic showing the steps involved in the successful implementation of the indirect drive approach to inertial confinement fusion (ICF) [2].

of these fusion events produces an $\alpha$-particle $\left({ }^{4} \mathrm{He}\right)$, a neutron, and $17.6 \mathrm{MeV}$ of energy.[124, 148, 123] If the DT fuel surrounding the initial 'hot spot' is sufficiently dense, the $\alpha$-particles will slow down due to collisions, converting their kinetic energy into thermal energy. 'Self-heating' occurs as this thermalization extends to regions surrounding the hot spot, increasing their temperatures to the point where fusion events begin to take place.

In this manner the region in which fusion events occur expands radially outward from the initial hot spot. If this propagation wave is self-sustaining, the plasma has 'ignited' and 'burns' as these fusion events trigger avalanches of fusion events in the surrounding regions of DT fuel, resulting in a net gain of energy such that $E_{\text {fusion }}>$ $E_{\text {laser }}$. Successfully achieving these conditions in the laboratory would represent a profound milestone in human history, opening the possibility of an unlimited source of energy on earth due to the abundance of hydrogen isotope 'fuel' present in the ocean. Given these consequences the pursuit of fusion energy by ICF processes is the primary driver of the research reported in this document.

The indirect drive approach to ICF was conceived by John Nuckolls in 1972 at the Lawrence Livermore National Laboratory (LLNL)[154]. A schematic of this process 
is shown in Fig. 1.1. Here, phenomenologically, energetic laser beams are focused onto the inside walls of a hohlraum, a centimeter-scale cylinder typically made of gold that houses a spherical capsule made of DT fuel and an outer 'shell.' The absorption of laser light by the hohlraum walls produces a quasi-Maxwellian 'bath' of x-rays that ablate the outer layers of the capsule, which are typically made from plastic or beryllium. Detailed images of the hohlraum and capsule design are shown in Fig. 1.2, highlighting the complex structure of the capsule. By analogy to rocket propulsion, the ablation of the capsule shell drives a shockwave into the DT fuel which heats and compresses it. The action of this shock heats the DT fuel to temperatures exceeding $>10^{8} \mathrm{~K}$ and compresses it to densities of $>100 \mathrm{~g} \mathrm{~cm}^{-3}$. These conditions cause the fuel to 'ignite,' creating a self-sustaining burn of nuclear fusion. For these typical density and temperature parameters, with 2 megajoules (MJ) of laser energy, the fusion energy yield is calculated to exceed $10 \mathrm{MJ}$ and $10^{19}$ neutrons over 10 100 ps, accounting for realistic losses of efficiency, e.g., less than unitary conversion from the laser energy to x-ray drive energy and radiation losses from the hohlraum through the laser entrance holes pictured in Fig. 1.2.[179] The gain factor is thus $G=E_{\text {fusion }} / E_{\text {laser }}=5 \cdot[148]$

LLNL has pioneered ICF research over the four decades since its inception. These efforts culminated with the construction of the National Ignition Facility (NIF) at LLNL in 2009[148, 122]. The scale of NIF is extraordinary: it is the most energetic laser system in the world by a factor of 100, and the facility itself covers the area of a stadium arena and contains more than 7,500 meter-sized optics and 30,000 smaller optics.[155,2] It is a 2 MJ, 500 terawatt (TW) laser system comprised of 192 laser beamlines. The laser beams originate from a 1051 nanometer wavelength (infrared) ytterbium-doped fiber laser having nanojoules of energy. These seed pulses are pre- 

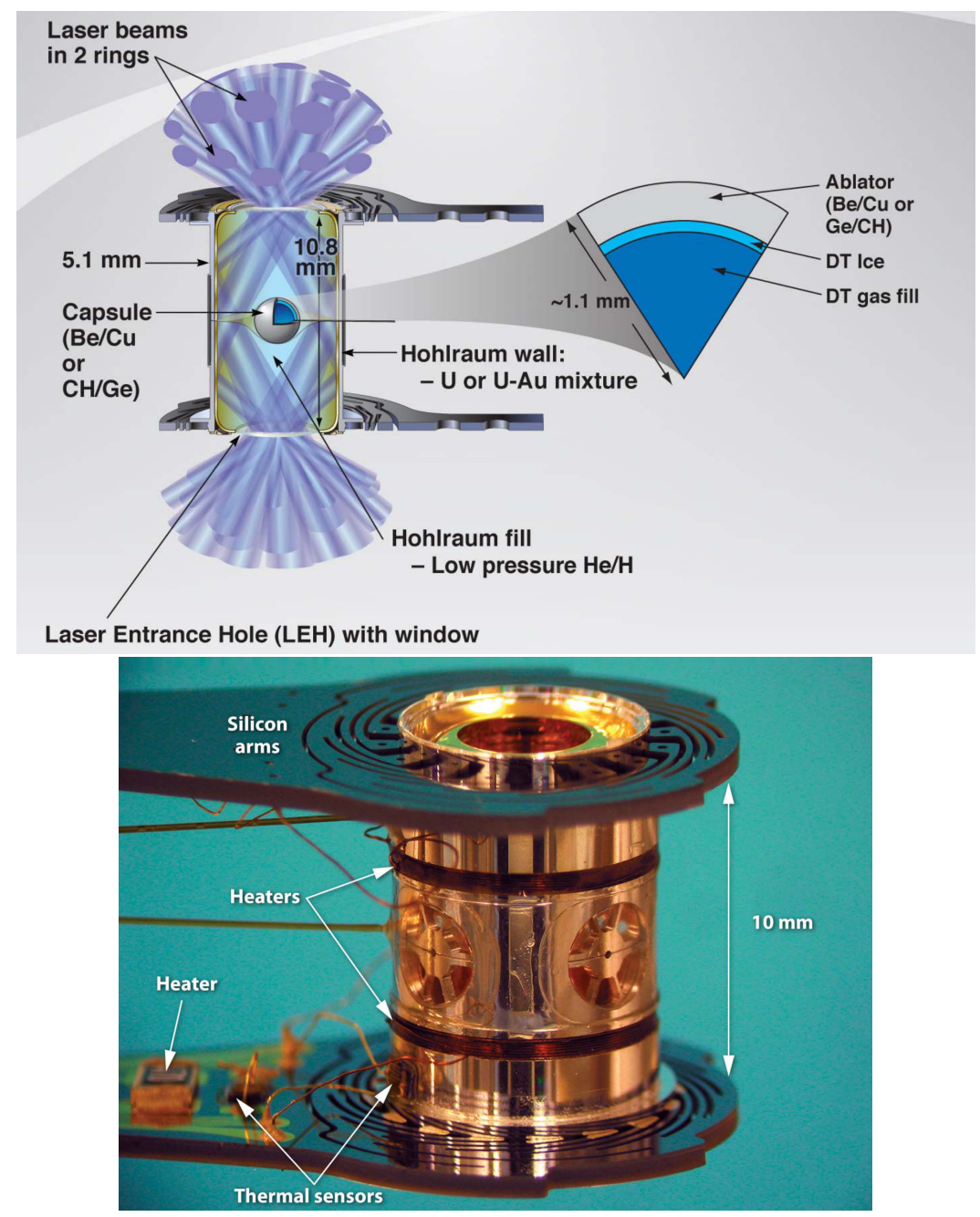

Figure 1.2 : The upper image shows a schematic of the hohlraum and fusion capsule suspended at its center. The bottom image shows this same setup, realized in cryogenic ICF experiments at the National Ignition Facility (NIF)[148, 2]. 
amplified, then passed six times through neodymium-doped glass amplifiers, resulting in an energy gain of a factor of $10^{15}$. Ultimately the facility is capable of producing 4 MJ of laser light at 1051 nanometer wavelength and $2 \mathrm{MJ}$ of light at 351 nanometer wavelength. [148, 122, 45] In ignition experiments the latter color is most often used since campaigns on the Nova laser in the 1990s showed that longer wavelength light coupled efficiently into supra-thermal electrons at the expense of the hohlraum x-ray radiation drive.[179]

For the virtues of the laser system, the National Ignition Campaign (NIC) on NIF between 2009-2012 seeking to achieve ignition was not successful[45]. While the NIC achieved several important milestones along this path[66, 65], a number of detrimental physics issues were also identified. These include the enhanced growth of hydrodynamic instabilities, among the most deleterious of which is the RayleighTaylor instability[141] occurring at the interface of the thin shell of the capsule, such as that depicted in the top panel of Fig. 1.2. Relating to these issues, significant progress has been made in the two year period since the the end of NIC in 2012[78, $158,172,99,41,42,45,44]$, and the promise of the indirect drive ICF approach to achieving fusion energy gain remains substantial.

\subsubsection{Fast ignition using high power lasers}

Alternative approaches to ICF, building on the indirect drive techniques described in section 1.1.1, have also been proposed. A key alternative approach invented by Max Tabak and colleagues at LLNL in 1994, termed the 'fast ignitor,' combines the indirect drive compression of DT fuel with an ultrafast petawatt laser 'ignitor' pulse.[204] Fast ignition (FI) supports the mitigation of hydrodynamic instabilities taking place during capsule compression and potentially offers a path to higher fusion gains. 


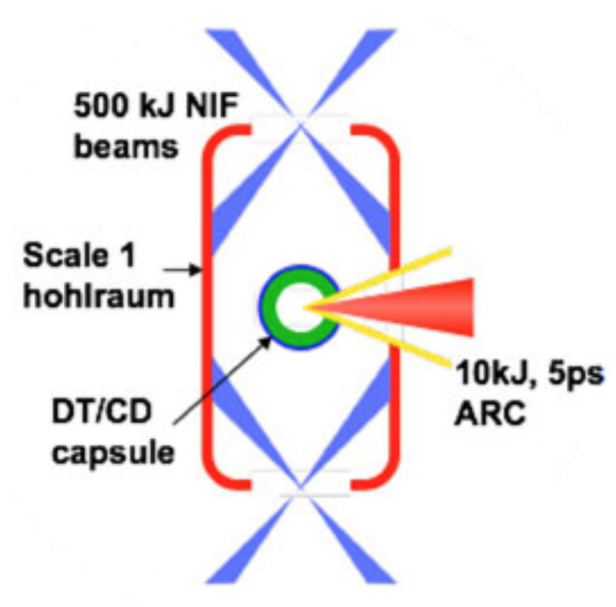

Figure 1.3 : Schematic of a fast ignition ICF hohlraum[2]. In this design a gold cone is used to guide the ultrafast ignitor pulse and shield its path from coronal plasma generated during the fuel compression stage of the process.

FI promises to achieve these benefits by decoupling the processes of fuel compression and fuel ignition.[204] In this approach first high energy lasers are shined on the walls of a holhraum, creating a bath of x-rays that ablate the outer layers of a DT fuel capsule, as described in section 1.1.1. The resulting ablation-driven shockwave compresses the DT fuel to a density $\rho \sim 300 \mathrm{~g} / \mathrm{cm}^{3}$ and temperature $5 \mathrm{keV}$ over several nanoseconds (ns). Then, at the time of peak compression, an ultrafast few - 10 picosecond (ps), high intensity $I_{l} \sim 10^{20} \mathrm{~W} / \mathrm{cm}^{2}, \sim 10 \mathrm{~kJ}$ laser is shined directly onto the imploded fuel, as shown in Fig. 1.3. This 'ignitor' laser pulse can be guided into the hohlraum and shielded from the coronal plasma generated during the fuel implosion by the use of a gold cone, as proposed by Peter Norreys and colleagues[153], and as illustrated in Fig. 1.3. If the short pulse laser can couple its energy to a region of radius $\sim 10 \mu \mathrm{m}$ of the DT fuel and heat it to $10 \mathrm{keV}$ before the fuel disassembles over $\sim 10 \mathrm{ps}$, the fuel will ignite.

Fusion energy gain by FI is strongly enhanced relative to conventional ICF, due 
to the fact that the latter scheme is isobaric: that is, the high-temperature lowdensity hot spot must be in pressure equilibrium with the low-temperature highdensity surrounding fuel. In FI the ignitor pulse is sufficiently fast that the hot spot and surrounding DT fuel need not equilibrate. The fusion gain $G$ associated with FI can therefore be calculated using an isochoric model in which both the hot spot and surrounding fuel have an equal, and significantly lower, density. By conservation of mass the FI scheme thus ignites having a larger volume of fuel to burn. It is for this reason that fusion gain in FI is enhanced; a comparison of $G$ between schemes is shown in Fig. 1.4. That the average density is lower in the FI approach also means that the compression is less, indicating a significantly reduced susceptibility to hydrodynamic instabilities, e.g. Rayleigh-Taylor, that occur at the capsule interface during the implosion of the fuel.[204]

Having established the contours and the advantages of the FI scheme it is instructive to review the physics challenges which must be overcome in order to demonstrate its viability. These relate to the coupling between the (optical) ignitor laser energy and the (optically-thick) fuel. A schematic of the relevant processes is shown in Fig. 1.5 .

Most often the coupling takes place using energetic, suprathermal electrons excited by the laser as a conduit for the electromagnetic energy. It is well known that as the ignitor laser strikes the tip of the gold cone, it interacts with the resulting fieldionized optically-thick plasma in a nonlinear fashion, generating a beam of relativistic 'hot' electrons with $>50 \%$ efficiency[215]. These electrons must propagate over an axial distance of $\sim 50-100 \mu \mathrm{m}$ from the cone tip, through the solid density gold of $\rho \sim 300 \mathrm{~g} / \mathrm{cm}^{3}$, into the imploded DT fuel. If the electrons do so in a collinear fashion, FI will succeed having large $G$ using only $\sim 10 \mathrm{~kJ}$ of ignitor pulse energy. 

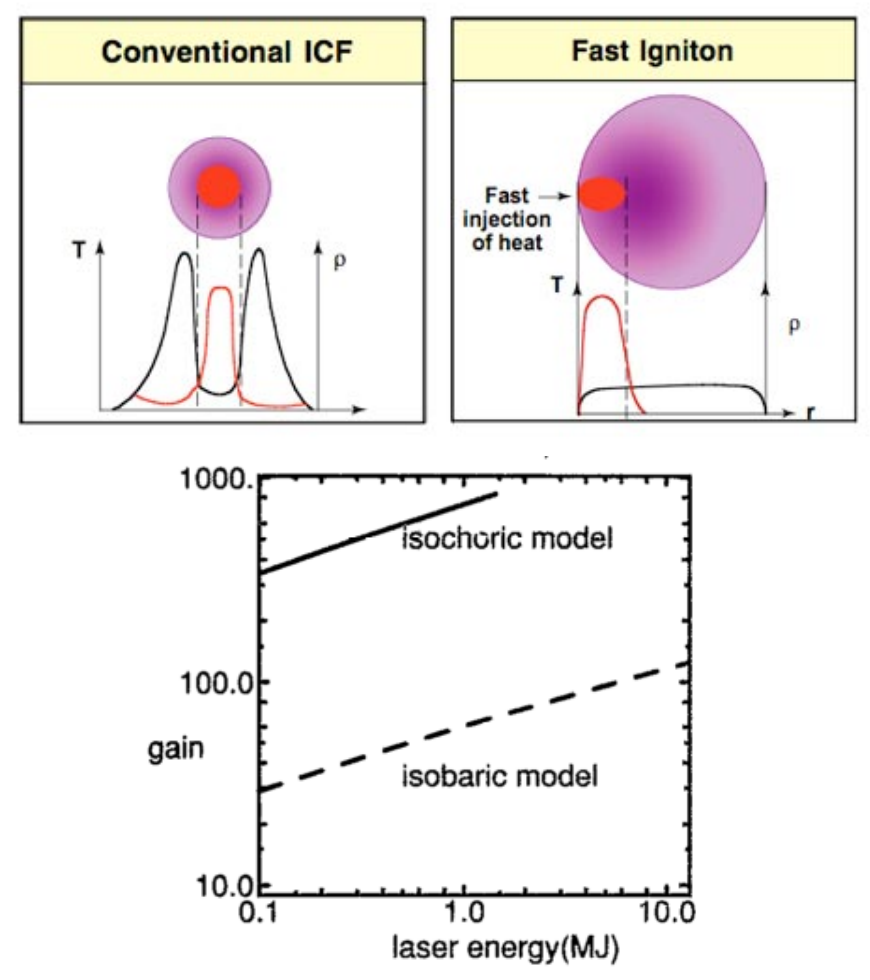

Figure 1.4 : Comparison between fast ignition and the conventional indirect drive ICF approaches. The left plot illustration the differences in density and temperature space[2]. The right plot indicates the ideal fusion gain curves $G$ for both the fast ignition (isochoric) and conventional (isobaric) approaches, with the lower axis corresponding to the laser energy driving the fuel compression.[204]. 
Hence, the main physics challenge is simply to induce electrons that are generated in a relativistic laser-plasma interaction to go straight over a distance of $\sim 50 \mu \mathrm{m}$ in dense matter.

The promise and conceptual simplicity of this situation have driven considerable theoretical and experimental efforts over the past two decades.[204, 93, 207, 81, 5, 9, $177,152,88]$ Yet, the bulk of research to date has indicated that electrons generated by a petawatt laser at an optically-thick interface do not naturally exhibit collinear trajectories with respect to the laser propagation axis, but rather substantial divergences. This has been shown with some clarity in experiment and through kinetic numerical simulations, but the primary underlying cause has eluded a clear description. That uncertainty is due to the complexity associated with the strongly nonlinear physics processes relevant to FI conditions. The core FI physics is also multi-scale in several important aspects: the relevant lengthscale for the physics of the interaction is the skin depth of the dense plasma $\sim 10^{-3}-10^{-2} \mu \mathrm{m}$; the laser wavelength $\sim 1 \mu \mathrm{m}$; the laser spot size $\sim 10 \mu m$; the electron transport distance from cone tip to the dense thermonuclear fuel is $\sim 100 \mu \mathrm{m}$; the laser frequency $\sim 1$ femtosecond $\left(\mathrm{fs}=10^{-15} \mathrm{~s}\right)$; and the laser pulse length $\sim 10 \mathrm{ps}$.

Spiting these disparate scales research performed over the past few years has shown promise in reducing the divergence of the hot electron beam produced by the laser. Schemes involving the tailoring of materials, such as focusing ellipsoids[9], magnetic 'switchyards' [176] and material resistivity matching[8], have been proposed to help collimate the electron beam, and have exhibited some successes. Other promising tracks of research have focused on understanding the initial absorption in detail; that is, the processes controlling the conversion of the ignitor laser energy into electron energy $[86,152,88,72,163,175,62,83,106,109]$. Germane research of this latter 

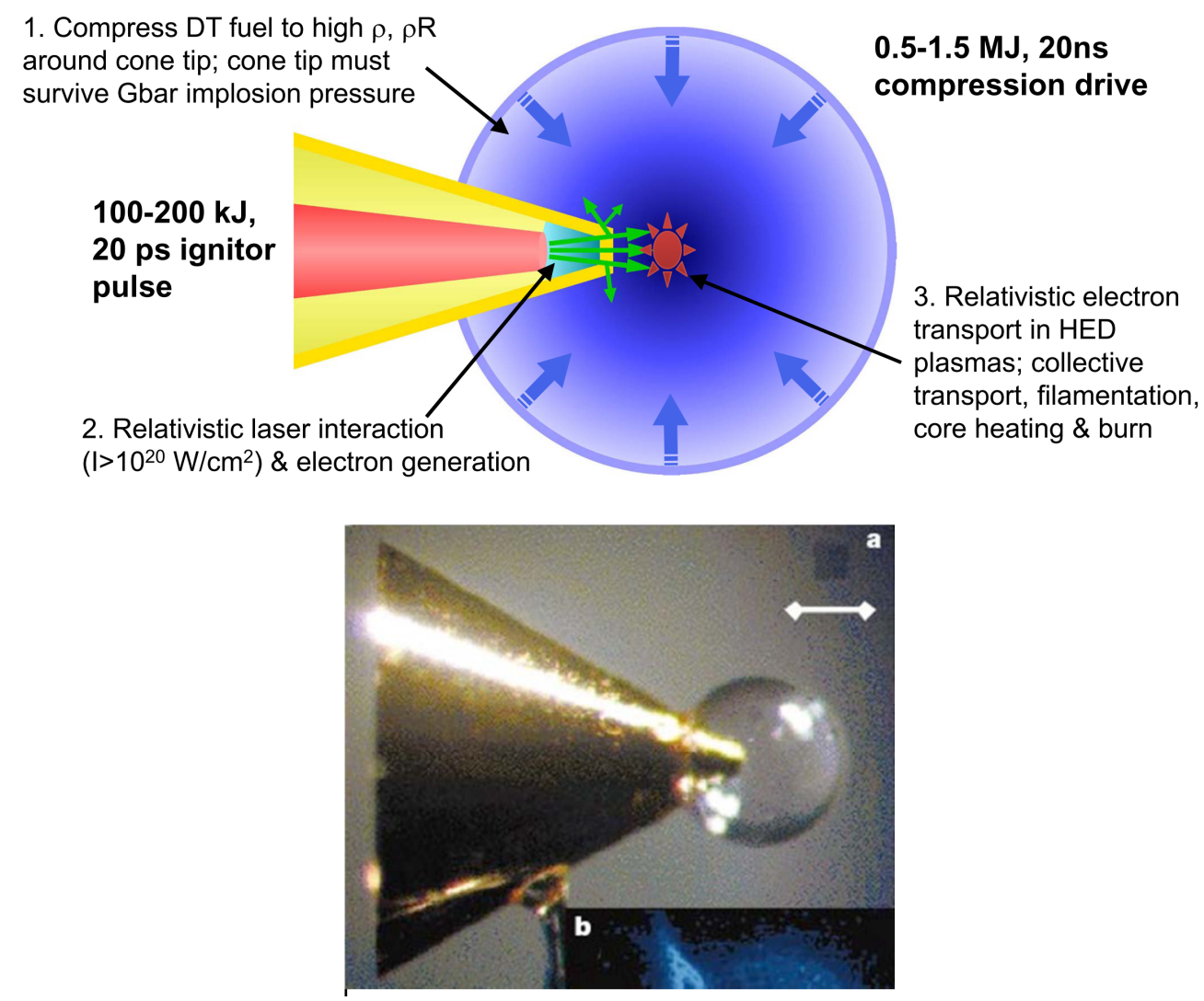

Figure 1.5 : Overview of the physics involved in the coupling of the ignitor pulse energy to the imploding DT fuel. The upper image shows a schematic which is realized in the lower experimental image.[57] In the latter image the transparent shell attached to the cone tip acts as a surrogate for the ignition DT fuel. 
sort is covered in chapters 3-4 of this thesis.

\subsubsection{Compact relativistic particle accelerators}

High energy ion accelerators have applications across many disciplines of science and medicine such as proton radiotherapy[25], isotopes for positron emission tomography[201] and inertial confinement fusion[204]. In the field of HED physics[34, 169, 43] the extreme photon power $(\mathrm{W})$ and fluence $\left(\mathrm{J} / \mathrm{cm}^{2}\right)$ from a laser irradiating a target can efficiently generate highly energetic ions. Through nanosecond-scale laser plasma interactions, the strong fields of the laser ablate the surface of the target, hydrodynamically driving plasma expansion with $\sim \mathrm{keV}$ ion kinetic energies[180]. With higher power, picosecond-scale ultraintense $\left(I \lambda^{2}>10^{18} \mathrm{~W} \mu \mathrm{m}^{2} \mathrm{~cm}^{-2}\right)$ laser pulses, research over the past few decades has identified nonlinear mechanisms[215] that can accelerate ions to $\mathrm{MeV}$ energies and beyond, including by means of the laser light pressure itself, as in a light sail[48]. In addition to the fundamental interest in eventually achieving $\mathrm{GeV}$-scale ions in compact accelerators, additional applications have opened in recent years to the emerging field of laboratory astrophysics, in which intense optical drivers enable the study of astrophysically-relevant phenomena such as collisionless shocks[53] and self-organization processes[98] in the laboratory.

Methods of energetic ion generation using ultraintense lasers interacting with high density targets carry advantages in terms of particle energy spectrum and scaling with laser driver energy. To date, the majority of research in this field has focused on two essential mechanisms[59]: 'hole punching' and 'target normal sheath acceleration' (TNSA)[216]. For the former, the pioneering work of Wilks et al.[215] identified the nonlinear ponderomotive force of the ultraintense laser, related to gradients in the laser field energy density and to relativistic electron current effects[95], as an efficient driver of ions. The essential physics described the laser fields coupling energy 
into electrons, accelerated forward under the excursion of time-averaged field energy gradients. A consequence of this nonadiabatic process is the generation of a strong space-charge field that acts on the ions, carrying them along and injecting them into the bulk target. A variety of applications for ions accelerated in this fashion have been studied, with the most common relating to FI ICF.[150] Over the last several years, there has been a resurgence of interest in a closely-related ion acceleration process called Radiation Pressure Acceleration (RPA)[48]. In effect, ions involved in the hole punching process may become accelerated to fractions of $c$ in a manner analogous to particles swept up and reflected from a moving wall: the ions are pushed into the bulk at approximately twice the hole punching velocity and propagate through the target. These ions may have a monoenergetic distribution depending on the plasma and laser intensity parameters, and the ion energy scales linearly with the laser intensity. An example phase space diagram for ions accelerated by hole punching is shown by Fig. $1.12(\mathrm{~d})$ in section 1.2.1 below.

The most widely utilized laser-based method of ion acceleration is referred to as target-normal sheath acceleration (TNSA)[216]. This method makes use of the ponderomotive 'hot' electrons, which are accelerated by the laser to multi-MeV energies, preferentially in the laser-normal direction. Because the hot electron collisional mean free path is long compared with the target spatial extent, these particles propagate largely unimpeded through the bulk. As they leave through the target-rear, a strong effective space-charge field that may exceed $\sim \mathrm{MeV} / \mu \mathrm{m}$ is set up. Ions along the target-rear become ionized as the laser-driven electrons exit, experience the sheath field, strip from the rear surface and subsequently accelerate. As the protons accelerated through the sheath effectively damp the field in a time-dependent manner, the resulting ion energies spread into a quasi-Maxwellian distribution, as shown in Fig. 

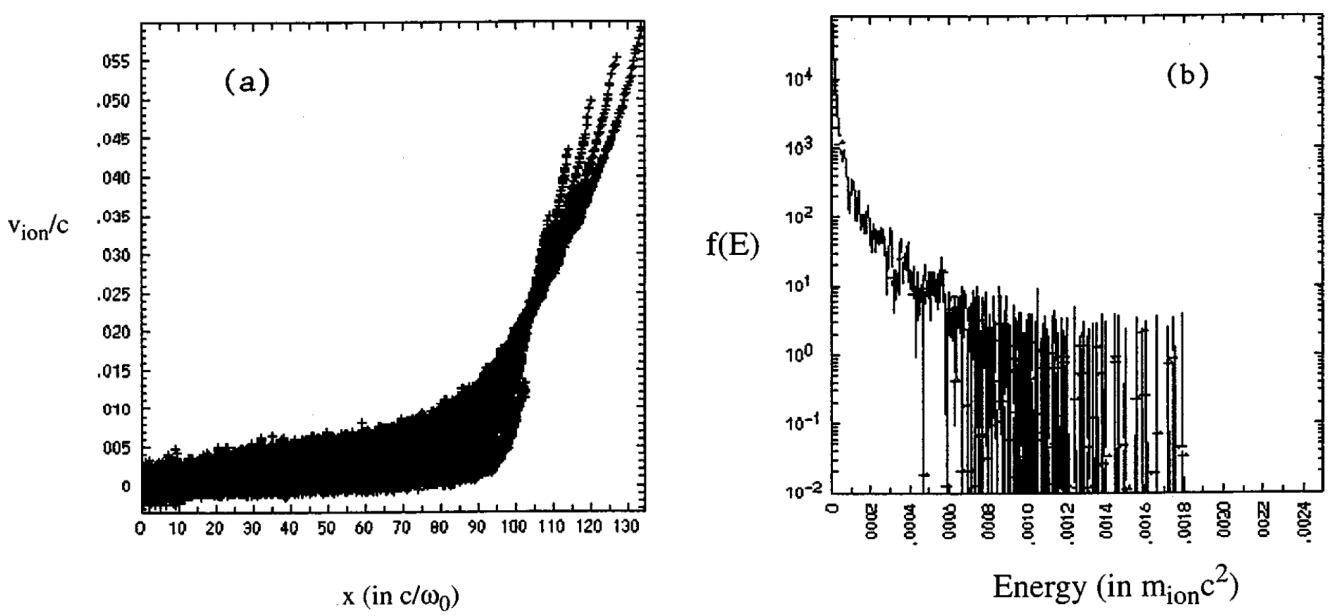

Figure 1.6 : Phase space (a) and energy distribution (b) of ions accelerated by the target normal sheath acceleration mechanism (TNSA), from ref. [216]. The peak ion energy in (b) reaches $4 \mathrm{MeV}$.

\section{6 .}

The multi-stage laser 'break-out afterburner' (BOA) mechanism has also been employed to accelerate ions with success. In this process a relativistic laser pulse having a time-varying amplitude over $\sim 1$ ps interacts with a classically-overdense target. That is, the target is opaque to the laser light having electron density $n_{0}$ satisfying $n_{0}>n_{c}$ where $n_{c}=m_{e} \omega_{l}^{2} /\left(4 \pi e^{2}\right)$ is the critical density for electron mass $m_{e}$, laser angular frequency $\omega_{l}$ and fundamental charge $e$. Initially the laser cannot propagate through the material, but as the laser strength increases, the target becomes relativistically transparent and the laser punches completely through the target, allowing the laser to co-propagate with ions and transferring its energy efficiently[74, 219]. Ions accelerated in this manner have been shown experimentally to exhibit a quasimonoenergetic spectrum as depicted in Fig. 1.7. Related to ion acceleration processes, the hole punching process is covered in more detail in chapter 3. 


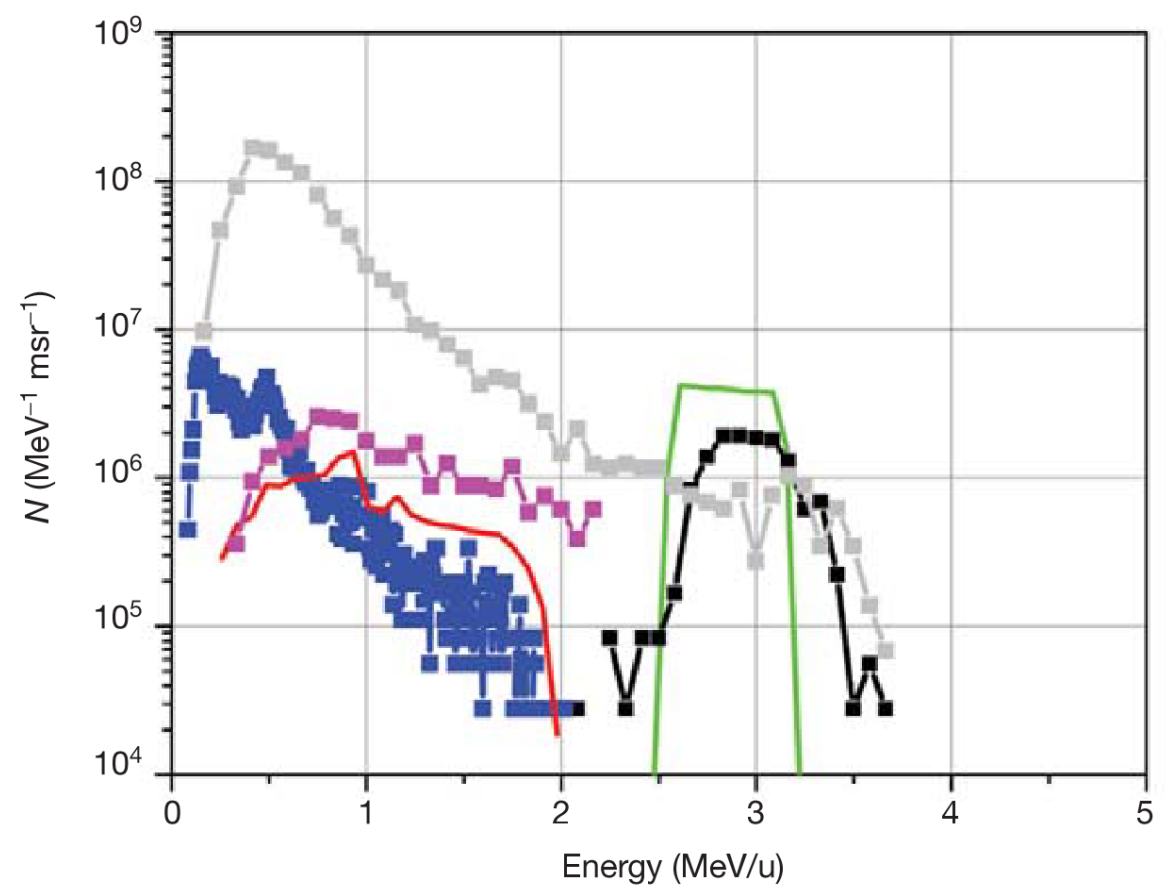

Figure 1.7 : Experimental spectra of ions accelerated by the TSNA and BOA mechanisms. The curves showing exponential-like spectrum are associated with the former process and quasi-monoenergetic spectra with the latter process, from ref. [74]. 

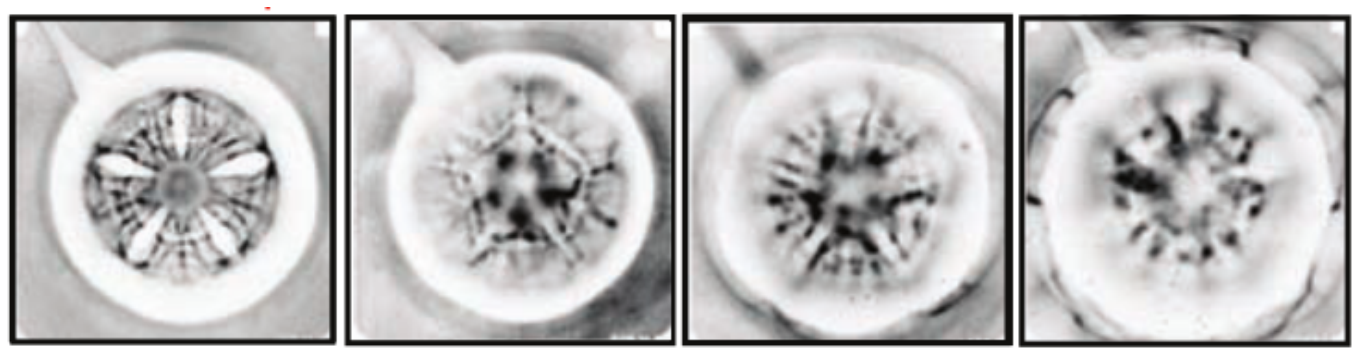

Figure 1.8 : Experimental proton radiography results showing megagauss-scale electromagnetic fields in inertial confinement fusion experiments, from ref. [115]. The spatial scale of each image is $2.9 \mathrm{~mm} \times 2.9 \mathrm{~mm}$. Panels show a time series of images captured at $0.85,1.6,2.17$ and $2.8 \mathrm{~ns}$, from left to right.

\subsubsection{Ultrafast imaging systems}

Understanding the electromagnetic field generation driven by intense laser-matter interactions is of fundamental importance to HED plasma physics $[34,169,43]$. In this pursuit the proton radiography diagnostic technique[76, 22, 15, 156, 23] has enjoyed considerable success, providing insight into megagauss-scale electromagnetic fields in inertial confinement fusion (ICF) implosions $[132,119,111,82,186,114,118,68$, 21, 190, 115, 137, 139, 138, 117, 224] , large-scale self-organizing electromagnetic field structures in high velocity counter-streaming plasma flows [97], magnetic reconnection processes[151, 113, 217], HED plasma instabilities[112, 77, 56, 61, 60] and more. Fig. 1.8 shows an instance of proton radiography of applied to these situations.

As implemented over the past decade, the proton radiography technique works by passing a low-density point-source-like proton beam through a HED plasma[182, $20,133,19,178,27,200,208,168]$. The proton beam is typically generated using the target normal sheath acceleration (TNSA) process in which an ultraintense short pulse laser $\left(>10^{18} \mathrm{~W} \mathrm{~cm}^{-2}\right.$ ) irradiates a solid target, producing a polychromatic proton source with useful energies ranging from $\sim 5-60 \mathrm{MeV}[216]$. Long pulse 
laser-driven implosions of $\mathrm{D}^{3} \mathrm{He}$ fusion capsules have also been employed to produce monoenergetic 3 and $14.7 \mathrm{MeV}$ proton sources [119, 111]. The protons generated using either process propagate ballistically from the source to the interaction region containing the HED plasma, deflect from the electromagnetic fields according to the Lorentz force, then travel ballistically to a distant detector where the radiograph, a two dimensional fluence map, is recorded. Radiography generated in this way is a uniquely high performance diagnostic, imaging HED plasmas with extraordinary spatial resolution of a few $-10 \mu \mathrm{m}$ and temporal resolution of $1-10$ ps.

Yet for the technique's virtues, the general question of how to interpret a radiograph in connection to its underlying electromagnetic fields has remained open. A key challenge stems from the fact that the radiographic image is not a one-to-one electromagnetic field map, but rather forms a convolution of the three dimensional fields with the sampling proton properties. Useful aspects of the field geometry have been deduced from qualitative inspection $[135,16,17,18,156,23,125,217,21,70,32]$, and by means of quantitative estimates based on scalings of the Lorentz force[79] when features of the plasma are known.[151, 113, 120, 186, 161, 189, 115, 116, 217, 218, 197, 224] Recently analytic theory describing the deconvolution has been developed[98], but its application is constrained to simple field geometries and low field strengths, since the general mapping is nonlinear and degenerate.

Related to this key outstanding issue in the application of petawatt laser technology to ultrafast imaging systems, a new simulation tool that interacts realistic laser-driven point-like proton sources with three dimensional electromagnetic fields of arbitrary strength and structure, using the discretized method, is described in chapter 6. Integrating elements of this section and of section 1.1.3, Fig. 1.10 in section 1.1.5 shows experimental images produced by the applying TNSA ion source to image astrophysically-relevant self-organizing electromagnetic field structures. 


\subsubsection{Laboratory astrophysics}

In recent years due to technological advances in laser technology and diagnostics it has become possible to create conditions relevant to astrophysical phenomena such as gamma-ray bursts (GRB) and their afterglows, supernova remnants[77, 56], and energetic antimatter-dominated plasma flows[28, 30, 191] in laboratory experiments for the first time.[34, 169, 43] Using shockwaves driven by nanosecond-scale lasers, results were recently reported that provide insight into possible mechanisms for the generation of the protogalactic magnetic field[69, 143]. Using these drivers to create high Mach number plasma flows further opens the door to studying collisionless shocks in the laboratory. Such shocks are believed to be ubiquitous in high-energy astrophysics, occurring in protostellar jets, supernova remnant shells, relativistic jets proximate to supermassive black holes in distant galaxies (i.e., in blazars and quasars), and gamma-ray bursts and their afterglows [14]. Recently the ACSEL (Astrophysical Collisionless Shock Experiments with Lasers) scientific collaboration has reported experimental observations of Weibel-generated plasma filamentation[209], a signature of the instability that is believed to underpin the formation of astrophysical collisionless shocks.[77] One representative image of this filamentation is reproduced in Fig. 1.9. Another recent success of laboratory astrophysics experiments has been the discovery of large-scale self-organization of electromagnetic fields in supersonic, laser-ablated counterstreaming plasma flows[97]. In ref. [97], self-organization is defined as the apparent inverse cascade of spatial scales, and accordingly energy scales, associated with the observed electromagnetic field distributions over time, from $\mu m$-scale at $2.2 \mathrm{~ns}$ to $\mathrm{mm}$-scale at $5.2 \mathrm{~ns}$. These results are reproduced in Fig. 1.10 and exhibit similarities with self-organization of magnetic fields observed in astrophysical contexts.[101] 


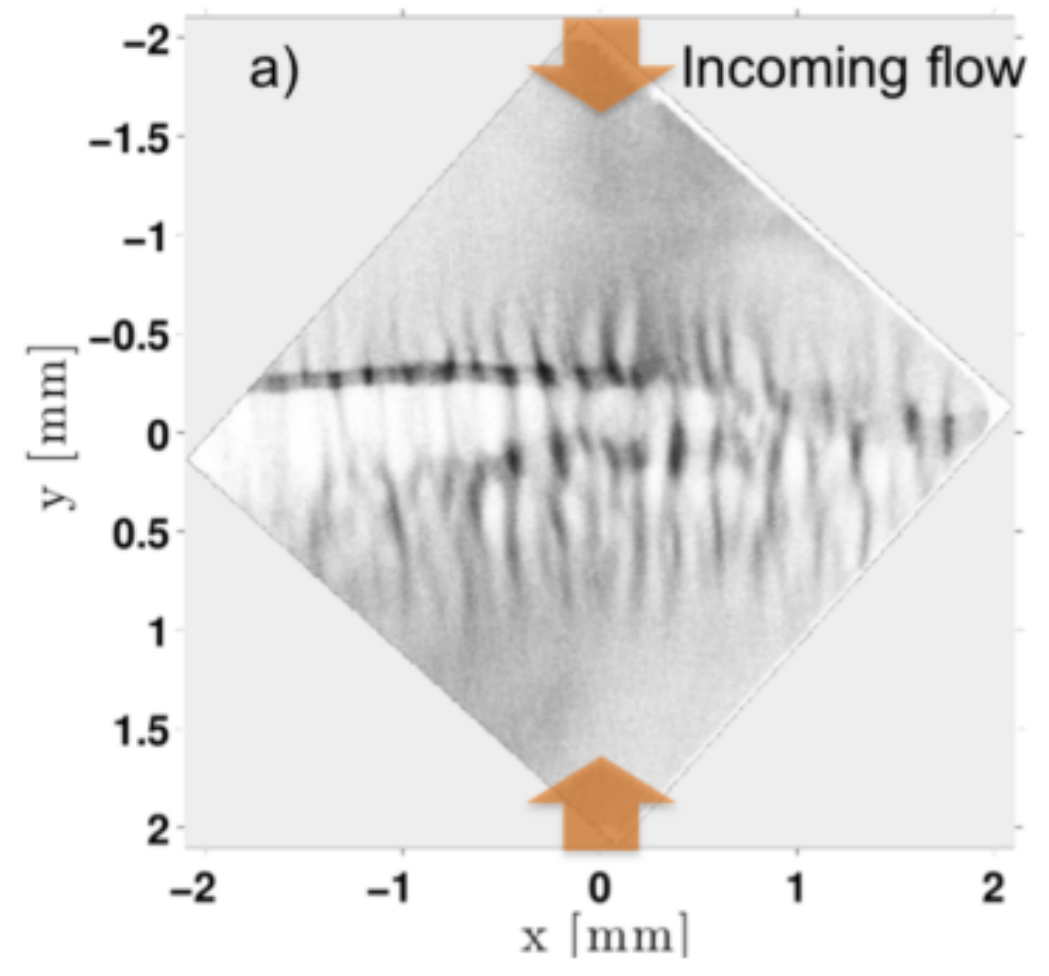

Figure 1.9 : Experimental proton radiography image of plasma filmamentation resulting from growth of the electromagnetic Weibel instability[209] in laboratory astrophysics experiments, from ref. [77] (details and experimental conditions found therein). 


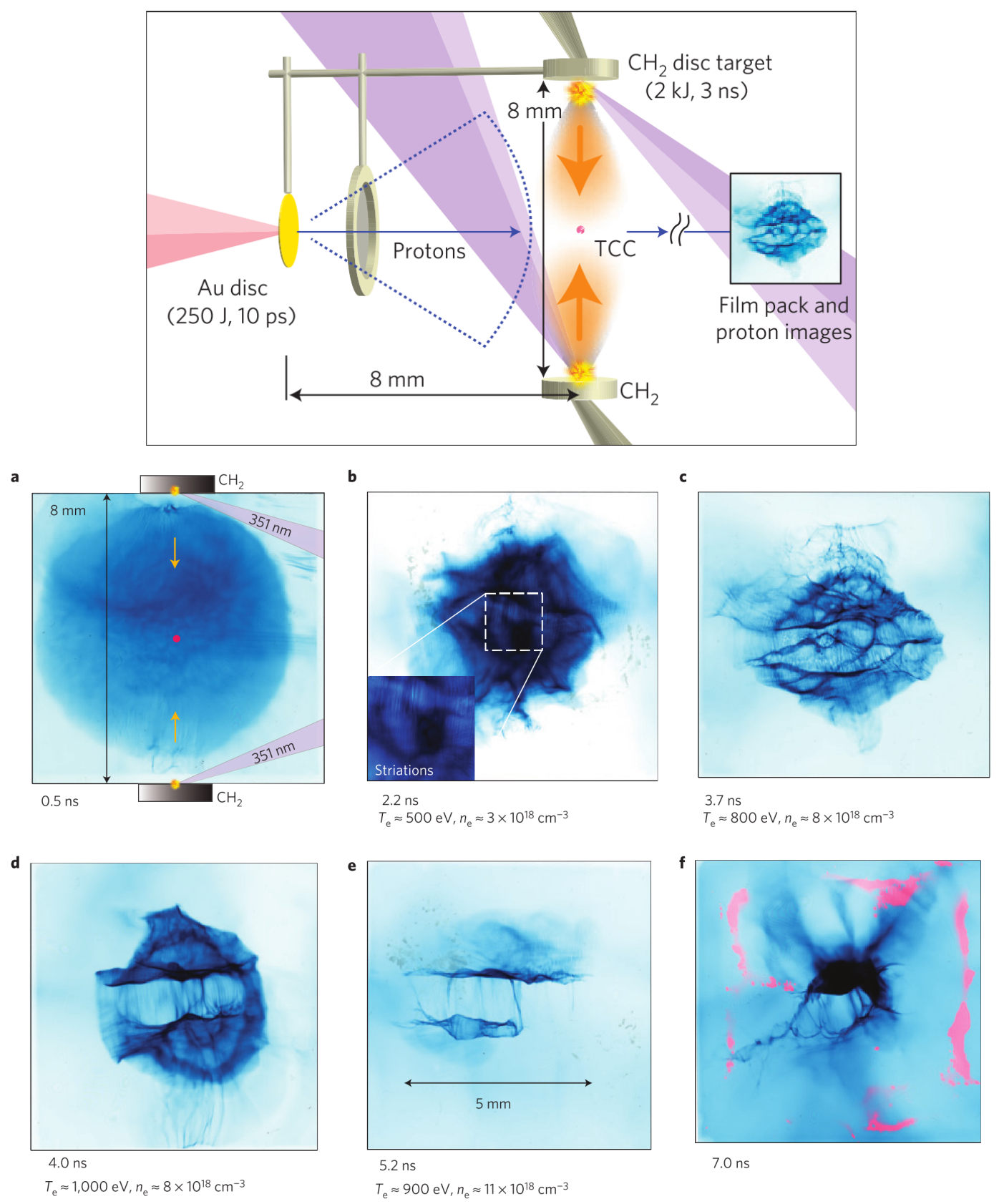

Figure 1.10 : Large-scale self-organization of electromagnetic fields in laboratory astrophysics experiments, from ref. [97]. The upper panel shows a schematic of the experiments in which high energy lasers are used to ablate two collinear plastic targets. The ablated plasma flows expand at supersonically, colliding with one another. The lower panel shows a series of experimental proton radiography image of self-organizing electromagnetic field structures emerging from this situation. 
More generally in ultraintense laser-plasma interactions, the structure of shocks driven into the target is intrinsically related to the laser driver and the mechanisms through which it couples energy into the plasma. One such important process is the laser pressure force, which enables hole boring[215] through classically overdense materials. The absorption of the laser light and its conversion efficiency into electrons and ions comprising the high density target is a key issue in studies of this topic. Relevant theoretical treatments are covered in section (1.2.1) and in chapters 3 and 4, and laboratory astrophysics experiments can play an important role in investigating these issues.

Notably, such conversion efficiencies are core unknowns in the study of astrophysical jets. There is a growing enthusiasm among astrophysicists for the paradigm that Poynting flux-dominated outflows in gamma-ray bursts (e.g. [126, 159]) and blazars (e.g. [221, 127] and references therein) drive their energization and dissipation at large distances from their central 'engines'. Most gamma-ray bursts (GRBs) are thought to emanate from powerful explosions of hypermassive stars located in distant galaxies in the early universe, at redshifts of around $z=1$ or larger $[165,144]$. A smaller number of so-called 'short' bursts may be the result of neutron star-black hole mergers. The energy release of $10^{51}-10^{54} \mathrm{ergs}$ is comparable to or somewhat higher than conventional supernovae in our Milky Way galaxy, but a key signature is that it drives a collimated, ultra-relativistic outflow (i.e., jet) with bulk Lorentz factors $\Gamma$ in excess of several hundred [4, 49]. Blazars are also extragalactic jet sources, but generally nearer by and less luminous than GRBs, and with inferences of less extreme bulk motions $(\Gamma \sim 3-50)$. They emanate from the environs of persistent supermassive black holes, exhibiting highly variable optical, X-ray [94] and gamma-ray emission, all the way up to a few $\mathrm{TeV}$ in photon energy. The variability in these wavebands can sometimes be as short as a few minutes, thereby indicating a compact physical 
scale of around $10^{13} \mathrm{~cm}$ for the emission/activation zone. The rapid flaring is most easily explained by interpreting the jets in blazars as being pointed almost directly towards an observer; relativistic Doppler effects then drive the large amplitudes of the flux fluctuations. Optical polarization measurements (e.g. see [50] for 3C 279) suggest that synchrotron emission from somewhat coherent magnetic field regions is what is seen in blazars, and this is the prevailing paradigm for non-thermal GRB jet emission also.

A key element of our understanding of both gamma-ray bursts and blazars is that the activation/emission region is not located right near the central engine, but is some distance/time further out. For bursts, the zone near the explosion event is Thomson optically thick to gamma-rays, and most of the emission we see does not resemble a blackbody spectrum. Therefore the radiative dissipation must arise predominantly outside the photosphere that is expected early in the expanding flow, and typically must arise at distance of $10^{15}-10^{17} \mathrm{~cm}$ from the 'hypernova' event. Similarly, blazars may become active only after their jets have been propagating for some time outside the black hole environs, an inference suggested by the optical polarimetry of synchrotron emission associated with gamma-ray flaring activity [50]. Accordingly, a core question for these topical sources is how is the energy transported out from the central region, and what is the most efficient means for doing so. It was realized long ago $[164,165]$ that pushing ions with the explosive force of a GRB progenitor star would lead to unreasonably large requirements for the energy of the explosion. This defined the so-called 'baryon-loading' problem for GRB jets, and led to the preferred paradigm of electron-positron pair jets composed of much lighter particles that are more easily accelerated to bulk speeds with $\Gamma>100$. The same is true for blazar jets. Yet what inhibits them from radiating efficiently until large distances from the central engine? This conundrum has precipitated the class of electromagnetic driver 
models $[126,159]$, where Poynting flux dominates the inner outflow zone in an inert mode, and converts to bulk kinetic energy and dissipates only after a while, perhaps via magnetic reconnection, thereby activating the jet particles so that they radiate the non-thermal gamma-rays and X-rays that we detect. It must be remembered that prevailing ideas concerning jet launching and propagation mostly require the presence of magnetic fields to effect continued collimation [146, 13]. Imbuing the jet with a dominant electromagnetic component is an efficient means for propagating energy out from the central regions and delaying the onset of radiative dissipation.

Understanding the efficiency of conversion of direct electromagnetic energy to plasma kinetic and thermal energies is therefore an extremely desirable advance. Astrophysicists modeling jet sources need to comprehend at greater depth how the electromagnetic energy is reassigned to electrons and ions. Laser-driven plasma interaction and associated kinetics can therefore provide crucial insights into these astrophysical phenomena. The development of laboratory astrophysics as a platform for these studies is an important step in this direction. These experiments, in concert with theoretical advances covered in chapters 3 and 4 of this document describing the ultimate kinematic apportionment of laser energy into efficient radiators, provide a first guide to how efficiently we think gamma-ray bursts and blazars can radiate if their outflows are mediated mostly by Poynting flux at early epochs in their expansion. Moreover, anticipating that down the line this study can address higher laser intensities, we can extend the focus to the relativistic flow speeds germane to bursts and blazars. This will then explore parameter regimes that precipitate rampant pair production, and therefore sample the domain of pair jets, perhaps the preferred picture for the later radiative phases of these highly variable astrophysical sources. An interesting potential future foray could be to explore multiple laser-plasma interaction sites corresponding an array of bulk flows, and these will in turn interact, forming col- 
lisionless shock zones, where charges will be energized and radiate the electromagnetic signals that we detect from GRBs and blazars.

\subsubsection{Probing the quantum vacuum structure}

As discussed in the preceding sections the interaction of high power lasers with matter forms the basis for promising applications in table-top particle accelerators[74, 3], ultrafast imaging systems [216, 98] and inertial confinement fusion.[204] These applications use current generation petawatt lasers with powers in the range of $I_{1} \lambda_{1}^{2} \sim$ $10^{18}-10^{21} \mathrm{~W} \mu \mathrm{m}^{2} \mathrm{~cm}^{-2}$, where $I_{1}$ is the laser intensity and $\lambda_{\mathrm{l}}$ is wavelength. Here, the laser power is sufficiently high that free electrons oscillating in the laser fields become relativistic, enabling efficient collisionless mechanisms of absorption. At fantastically high powers $\sim 10^{29} \mathrm{~W} \mu \mathrm{m}^{2} \mathrm{~cm}^{-2}$, the laser electric field approaches the quantum critical 'Schwinger' field[194],

$$
E_{\mathrm{c}}=\frac{m_{e}^{2} c^{3}}{e \hbar} \simeq 1.3 \times 10^{18} \frac{\mathrm{V}}{\mathrm{m}}
$$

opening the possibility of directly testing nonlinear properties of the quantum vacuum. This section describes the physical processes occurring in this intensity interval and the implications thereof.

At the upper bound of this intensity interval, $\sim 10^{29} \mathrm{~W} \mu \mathrm{m}^{2} \mathrm{~cm}^{-2}$, the electric field of the laser given by equation (1.1) is sufficiently strong to accelerate an electron to the energy $2 m_{e} c^{2}$ over the Compton wavelength. At this strength the field can rip apart the vacuum itself and trigger a cascade of formerly virtual electron-positron pairs. This Schwinger mechanism[194] is believed to form the ultimate upper limit on light which can exist in the universe. However, this threshold is seven orders of magnitude above the highest power laser pulse on record and reaches far beyond the 
limits of modern laser amplification technology. Therefore, if lasers are to access this intriguing domain, sophisticated physics-based techniques are clearly needed.

The first such physics process, proceeding through nonlinear quantum electrodynamical (QED) methods, was developed in ref. [121]. This work predicted that electron-positron pairs would be generated in ultraintense laser interactions with matter at intensities $>10^{19} \mathrm{~W} \mu \mathrm{m}^{2} \mathrm{~cm}^{-2}$. Crucially, pair generation through the processes outlined in [121] require relativistic electrons excited by the laser as 'seed' particles responsible for initiating QED anti-matter creation. Such pairs are believed to be essential constituents in numerous high-energy astrophysical events such as pulsar winds, blazar jets, and gamma-ray burst outflows, they also are also intimately connected to the fundamental nature of light and its conversion into matter.

Recent work has shown the Schwinger mechanism can generate positrons at much lower intensities, perhaps $10^{24} \mathrm{~W} \mu \mathrm{m}^{2} \mathrm{~cm}^{-2}$ corresponding to a $\sim 10 \mathrm{PW}$ class laser system. This is due to the fact that the electric field as experienced by ultrarelativistic electrons can be significantly upshifted in their rest frame[7]. The Trident and Bethe-Heitler QED processes also comprise key avenues by which laser light can be absorbed and converted into positrons. While experiments have demonstrated copious pair production through these latter mechanisms, $\sim 10^{10}$ pairs, the total amount of energy converted into anti-matter is $<1 \%$ due to the limitations on existing laser technology[28, 30]. The physics basis for this research is covered in section 1.2 .4 .

Another technique based on relativistic laser-plasma interactions promising to achieve fields on the order of the quantum critical field at next generation laser facilities is referred to as coherent harmonic focusing (CHF) [67]. High harmonics (light with wavelength $\lambda_{n}=\lambda_{\mathrm{l}} / n$ for the $\mathrm{n}$-th harmonic) are generated as the laser-matter interface undergoes violent oscillations during irradiation by a petawatt laser, exhibit- 
ing a slowly-decaying power law spectrum extending out to very large $n[67]$. These oscillations cause portions of the laser light wave reflected from the interaction medium to undergo very large coherent, Doppler-like upshifts in frequency. In the promising CHF mechanism, these reflected high harmonics are geometrically focused down into a sub-attosecond burst of ultra-intense coherent light. The focused intensity is predicted to scale as $I_{1}^{5 / 2}$, reaching the quantum critical field at $I_{1} \sim 9 \times 10^{22} \mathrm{~W} \mathrm{~cm}^{-2}$ for $1 \mu m$ wavelength light, about an order of magnitude higher than has been achieved at current laser facilities. 


\subsection{Theory of intense laser-plasma interactions}

The extreme states of matter that are created in petawatt laser-plasma interactions reach temperatures exceeding ten million degrees Celsius and pressures exceeding one billion earth atmospheres. These high energy density conditions are driven at the microscopic scale by dense currents of relativistic electrons $\left(\sim 10^{11} \mathrm{~A} \mathrm{~cm}^{-2}\right)$, oscillating violently in the intense laser fields $\left(>10^{10} \mathrm{~V} \mathrm{~cm}^{-1}\right)$, as well as the plasma processes arising when these particles are dephased and injected into the high density target.[129] In studies of this topic, the interaction begins with the transfer of laser energy to particles through complex mechanisms. Yet, because the conditions of illumination are so nonlinear, an outstanding problem for the high power laser community has been to predict the amount of laser light absorbed and deposited in the form of heat in electrons and ions, plasma turbulence, and in energizing non-thermal particles. This theory section is therefore presented with emphasis on topics relating to absorption, which is the focus of a substantial portion of the advances offered in this thesis.

\subsubsection{Particle dynamics in ultraintense light fields}

The interaction of ultraintense laser light having normalized vector potential,

$$
a_{0}=\frac{|e| E_{l}}{m_{e} c \omega_{l}}=\frac{p_{o s c}}{m_{e} c}
$$

with matter is characterized by the nonlinear action of the light[215]. In this expression $e$ is the fundamental charge, $E_{l}$ is the electric field of the laser pulse, $m_{e}$ is the electron mass, $c$ is the speed of light, $\omega_{l}$ is the laser angular frequency and

$p_{\text {osc }}$ is defined as the electron oscillatory momentum. $[215,214,130]$ The nonlinear 
ponderomotive force of the light is described by [95, 202],

$$
\overrightarrow{f_{p}}=\vec{\nabla}(\gamma-1) m_{e} c^{2}
$$

for electron Lorentz factor $\gamma^{2}=1+\left(p /\left(m_{e} c\right)\right)^{2}$, momentum $\vec{p}=\gamma m_{e} \vec{v}$ and velocity $\vec{v}$. The force on an electron given by equation (1.3) is nonlinear due to the $\vec{v} \times \vec{B}$ term of the Lorentz force,

$$
\frac{d \vec{p}}{d t}=-e\left(\vec{E}+\frac{\vec{p}}{\gamma m_{e} c} \times \vec{B}\right)
$$

where $\vec{E}$ and $\vec{B}$ are the electric and magnetic fields. Since the electrons move near the speed of light, typically $\mathcal{O}(e E)=\mathcal{O}(e \vec{v} / c \times \vec{B})$, leading to first-order nonlinearities in the electron equations of motion. This fact is principally responsible for the richness of ultraintense laser science, as we shall see in this and later sections.

By convention theoretical topics in ultraintense (also known as relativistic, petawatt and high power) laser physics are formulated in terms of the laser intensity (irradiance),

$$
I_{l}=\left\langle\left|\vec{S}_{l}\right|\right\rangle=\frac{c}{4 \pi}\left\langle\vec{E}_{l} \times \vec{B}_{l}\right\rangle=\frac{c E_{l}^{2}}{8 \pi}=\frac{a_{0}^{2} m_{e}^{2} c^{3} \omega_{l}^{2}}{8 \pi e^{2}}
$$

where $\vec{S}_{l}$ is the laser Poynting flux, $|\cdot|$ denotes magnitude and $\langle\cdot\rangle$ denotes time-average. These relations yield a useful formulation of the relativistic threshold,

$$
a_{0}=0.85 \sqrt{\frac{I_{l} \lambda_{l}^{2}}{10^{18} \mathrm{~W} \mu \mathrm{m}^{2} \mathrm{~cm}^{-2}}}
$$

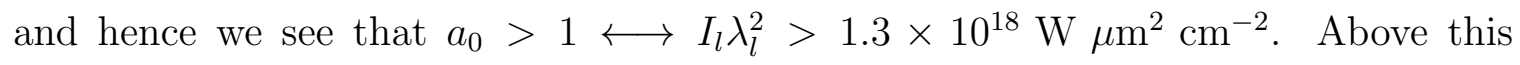
threshold Coulomb collisions are typically negligible, since $\lambda_{m f p} / \ell \ll 1$ where $\ell$ is 
the lengthscale associated with the region containing the laser fields, and the standard ICF-relevant theoretical models assuming Maxwellian particle distributions are undermined.[179, 124] This motivates, and often necessitates, particle-in-cell (PIC) simulation tools that capture the kinetic nature of the dynamics, as will be discussed in chapter 2.

Fig. 1.11 provides an important measure of context to the relativistic condition in equation (1.6). The development of short pulse laser technology and focusing techniques such as chirped-pulse amplification (CPA)[203] have enabled production of petawatt laser systems with intensities exceeding $>10^{21} \mathrm{~W} / \mathrm{cm}^{2}$ to date. The theory developed in this section covers laser interactions in the petawatt regime[129], i.e., in the intensity interval $10^{18} \mathrm{~W} \mu \mathrm{m}^{2} \mathrm{~cm}^{-2}<I_{l} \lambda_{\mathrm{l}}^{2}<10^{23} \mathrm{~W} \mu \mathrm{m}^{2} \mathrm{~cm}^{-2}$. The following section, section 1.2.4, presents a consideration of interactions at the 10petawatt scale, i.e., $>10^{23} \mathrm{~W} \mu \mathrm{m}^{2} \mathrm{~cm}^{-2}$.

At the petawatt scale the laser ponderomotive force $\vec{f}_{p}$ couples the incident photon flux into two primary kinetic modes: (1) 'hole boring' or 'hole punching' ions accelerated by the space-charge force associated with electrons under the excursion of time-averaged field energy gradients; and (2) relativistic 'hot' electrons excited by the oscillatory component of the ponderomotive force. These dynamics are exhibited by the following expression of $\vec{f}_{p}$ related to equation (1.3). Following ref. [95], for simplicity consider an electrostatic field of the form $\vec{E}_{l}=E_{l} \sin \omega_{l} t \hat{x}$, where $\hat{x}$ is the transverse direction, impinging upon a high density plasma of $n_{0}>n_{c}$ at $z=0$. Here $n_{0}=n_{p e}$ is the plasma electron density and $n_{c r}=m_{\mathrm{e}} \omega_{1}^{2} /\left(4 \pi e^{2}\right)$ is the critical density at which the plasma becomes opaque to the laser light. The longitudinal 


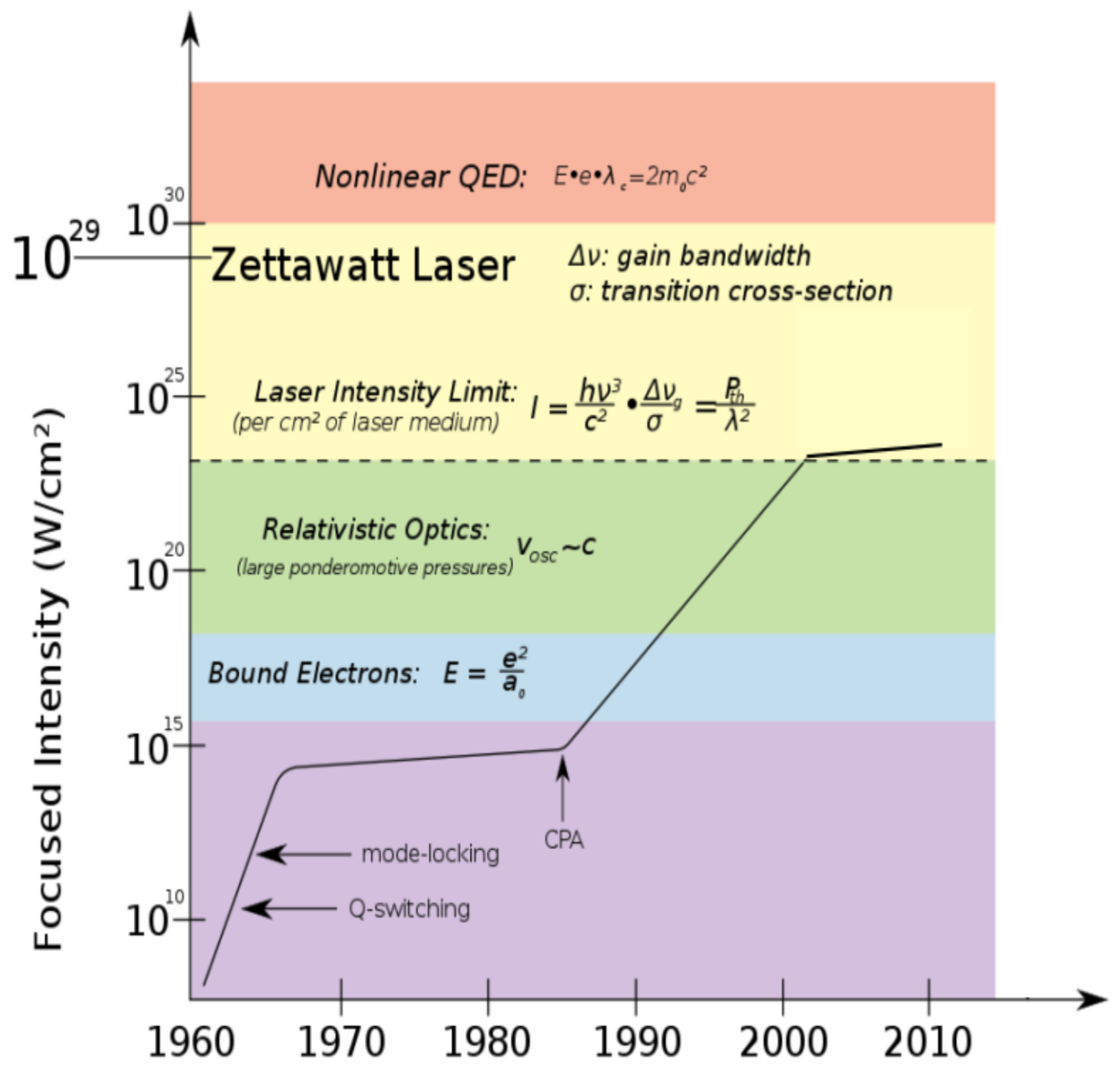

Figure 1.11 : Progression of intense laser technology over time, adapted from ref. [212]. The chirped-pulse amplification (CPA) technique[203] is seen to be responsible for enabling the rapid growth of high intensity laser systems, extending into the petawatt regime. 

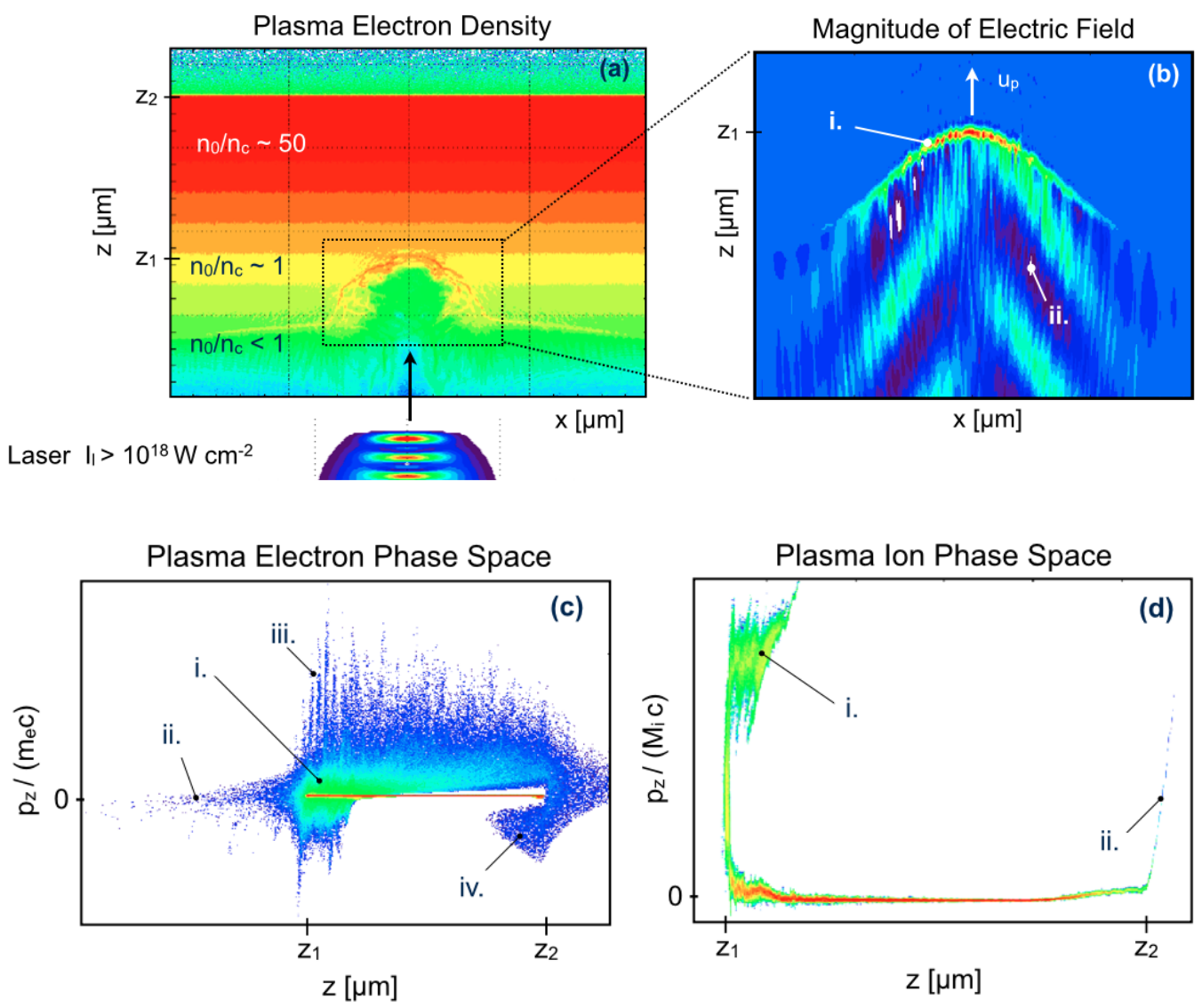

Figure 1.12 : Depictions of key features of petawatt laser-plasma interactions from two dimensional particle-in-cell simulations. $z_{1}$ corresponds to the current axial position of the laser-matter interface and $z_{2}$ corresponds to the target rear; scales have been omitted to emphasize the generality of the features across typical conditions. (a) shows a configuration space map of the plasma electron density during the period of laser irradiation. The electric field magnitude in the region containing the laser is shown in (b). The color scale in this plot progresses from blue to green to red as the field strength increases. (c) and (d) depict the electron and ion phase space in the simulation (see text). 
ponderomotive force is given by,

$$
\overrightarrow{f_{p}}=-\frac{m_{e}}{4} \frac{d}{d z} a_{0}^{2}\left[1-\cos \left(2 \omega_{l} t\right)\right] \hat{z}
$$

describing the $j \times B$ absorption mechanism of Kruer et al.[95] From this expression both the steady-state component $\left\langle\vec{f}_{p}\right\rangle$ and the oscillatory $\vec{f}_{p}-\left\langle\vec{f}_{p}\right\rangle$ component of the ponderomotive force are evident. It is further clear that electrons are accelerated by the laser into the plasma with a characteristic frequency of $2 \omega_{l}$.

Exhibiting these features, the electron and ion dynamics from a petawatt laserplasma interaction are shown in Fig. 1.12. We have generated these plots from a high resolution PIC simulation using the LSP code[211], and the axis values have been removed to highlight the fact that the simulation is representative of a broad range of typical laser-plasma conditions. In this simulation laser light enters the box at the lower $z=0$ boundary and is incident upon an fully-ionized plasma having an exponential density profile, with typical values labeled in (a). This density profile is used to model the effects of 'pre-plasma:' driven by intrinsic amplified spontaneous emission (ASE) and other processes involved in laser pulse compression[203], the bulk heating and hydrodynamic response often results in the formation of a largescale underdense shelf, or pre-plasma, in front of the nominal target surface[134]. Signatures of both modes of the nonlinear force are evident in panels (a-d); in the following sections these signatures in connection to electron and ion dynamics will be discussed in detail. 


\subsubsection{Acceleration of high density plasma due to the steady-state pon- deromotive force}

As shown in Fig. 1.12 (a) the steady-state component $\left\langle\vec{f}_{p}\right\rangle$ of the force in equation (1.7) is sufficiently strong that it punches a hole into the dense plasma target. Hole punching is a ubiquitous feature of petawatt laser-plasma interactions and was first predicted by Wilks et al. in 1992.[215] The kinetic structure set up by $\left\langle\vec{f}_{p}\right\rangle$ at the front of the laser pulse is referred to as the 'laser piston,' and its signature space-charge is evident in label (i) in panel (b). The imprint of the laser is shown by label (ii). The velocity at which the laser piston punches into the target, the hole punching velocity $u_{p}$, can be estimated through a momentum balance between the laser light and the ions accelerated by the piston as,

$$
\begin{aligned}
M_{i} n_{i} u_{p}^{2} & =\frac{I_{l}}{c} \\
\frac{u_{p}}{c} & =\sqrt{\frac{Z m_{e} n_{c}}{2 M_{i} n_{p e}}} a_{0} \equiv \beta_{0}
\end{aligned}
$$

where $M_{i}$ is the plasma ion mass, $n_{i}$ is the plasma ion density and $Z$ is the plasma average charge state. Electronic dynamics are implicit in this relation, as the laser couples its energy and momentum primarily into electrons, sweeping them into the target and creating a space charge electrostatic potential that is responsible for the acceleration of ions $[215,150]$. Below we examine modern ion dynamic models. For comparative purposes, models of ion dynamics presented in this section will be formulated in terms of the Wilks model result for the dimensionless piston speed $\beta_{0}$, in accordance with equation (1.9). Contours of this essential parameter are shown in Fig. 1.13.

As a consequence of the laser propagating into the target, background plasma ions 


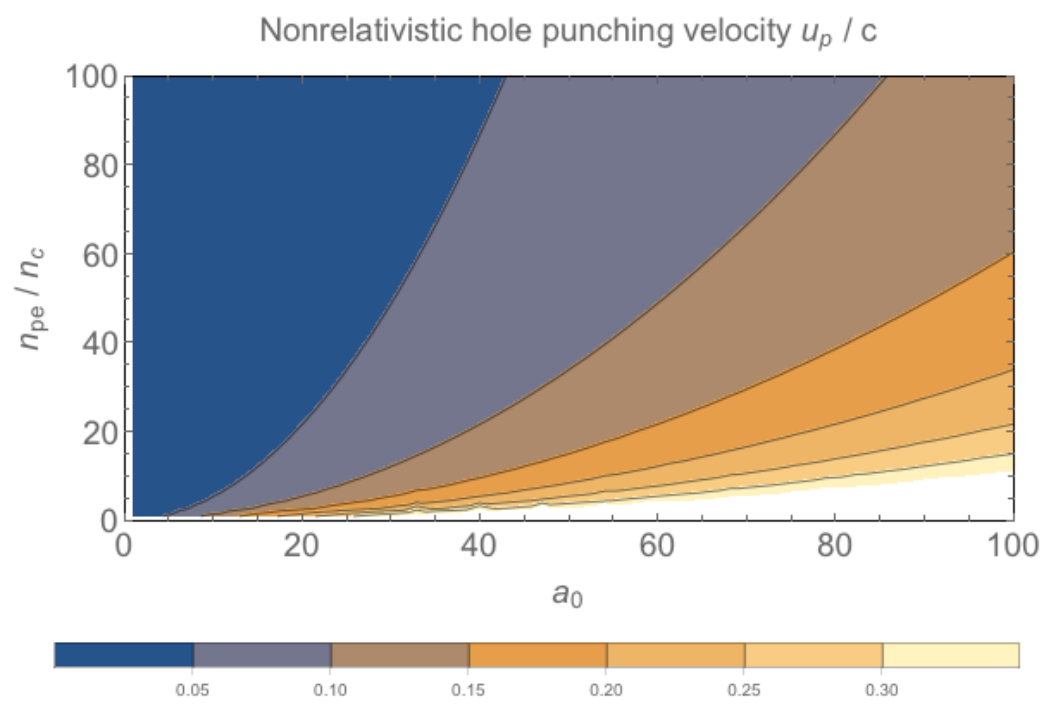

Figure 1.13 : Contours of $u_{p} / c$, the rate at which a petawatt laser punches into a high density target, as a function of the normalized laser intensity and electron plasma density.

are picked up and reflected by the electronic space charge structure comprising the laser piston. These ions can be accelerated to very high energies, $\mathcal{E}_{i}=1 / 2 M_{i}\left(2 u_{p}\right)^{2} \gg$ $\mathrm{MeV}$ even when the piston velocity is nonrelativisic, i.e., $u_{p} / c \ll 1$. Since $u_{p} / c \propto$ $\sqrt{Z m_{e} / M_{i}}$ while $\mathcal{E}_{i} \propto M_{i}$, this effect is enhanced for heavier ions such as Carbon. In the phase space depicted in Fig. 1.12 (d), label (i) shows ions that have been accelerated by the laser piston. The fraction of the laser energy absorbed in this process $f_{i}$ can be estimated as,

$$
\begin{aligned}
f_{i} I_{l} & =2 M_{i} n_{i} u_{p}^{3} \\
f_{i} & \simeq 2 \frac{u_{p}}{c}
\end{aligned}
$$

where $f_{i}$ can also be considered the laser conversion efficiency into ions[214].

The hole punching model was extended to relativistic piston velocities by Naumova 
et al.[150] The calculations in this work proceed in a frame of reference co-moving with this piston which we denote here using primes. In this frame the laser intensity is Doppler shifted as $I_{l}{ }^{6}=\left(1-\beta_{p, N}\right) /\left(1+\beta_{p, N}\right) I_{l}$ for dimensionless relativistically-correct piston velocity $\beta_{p, N}$. Conservation of mass flux can then be written as $2 c^{2} \gamma \beta_{p, N}^{2} M_{i} n_{i}^{\prime}=$ $2 c^{2} \gamma^{2} \beta_{p, N}^{2} M_{i} n_{i}$. Momentum flux conservation then yields,

$$
\begin{aligned}
\frac{1-\beta_{p, N}}{1+\beta_{p, N}} \frac{I_{l}}{M_{i} n_{i} c^{3}} & =\gamma^{2} \beta_{p, N}^{2} \\
\beta_{p, N} & =\frac{\beta_{0}}{1+\beta_{0}}
\end{aligned}
$$

The absorption fraction into ions associated with equation (1.13) can be calculated as,

$$
f_{i}=\frac{2 \beta_{p, N}}{1+\beta_{p, N}}
$$

Comparisons of the piston velocities in equations (1.9) and (1.13) are shown in Fig. 1.14. From this figure it is clear that the results of Naumova et al.[150] can be considered to be the relativistic corrections to those of Wilks et al.[215]. By the field's convention we evaluate this correction in terms of the piston velocity, $\Delta \beta$, and we note that $\Delta \beta$ becomes important only at very high laser intensities. $\Delta \beta=\left(\beta_{0}-\beta_{p, N}\right) / \beta_{0} \simeq \beta_{0}$, hence, the correction reaches $10 \%$ at $\beta_{0}=0.1$; for a laser interacting with a plasma having relativistically-opaque density $n_{0}=a_{0} n_{c r}$, this point corresponds to a laser having intensity $\sim 7 \times 10^{21} \mathrm{~W} / \mathrm{cm}^{2}$ at $1 \mu \mathrm{m}$ wavelength. Piston velocity calculations for a variety of situations, such as oblique laser incidence, have been considered in refs. [72, 163, 192, 175, 83, 106]. Further extensions to these results are presented in this document in chapters 3 and 4. 


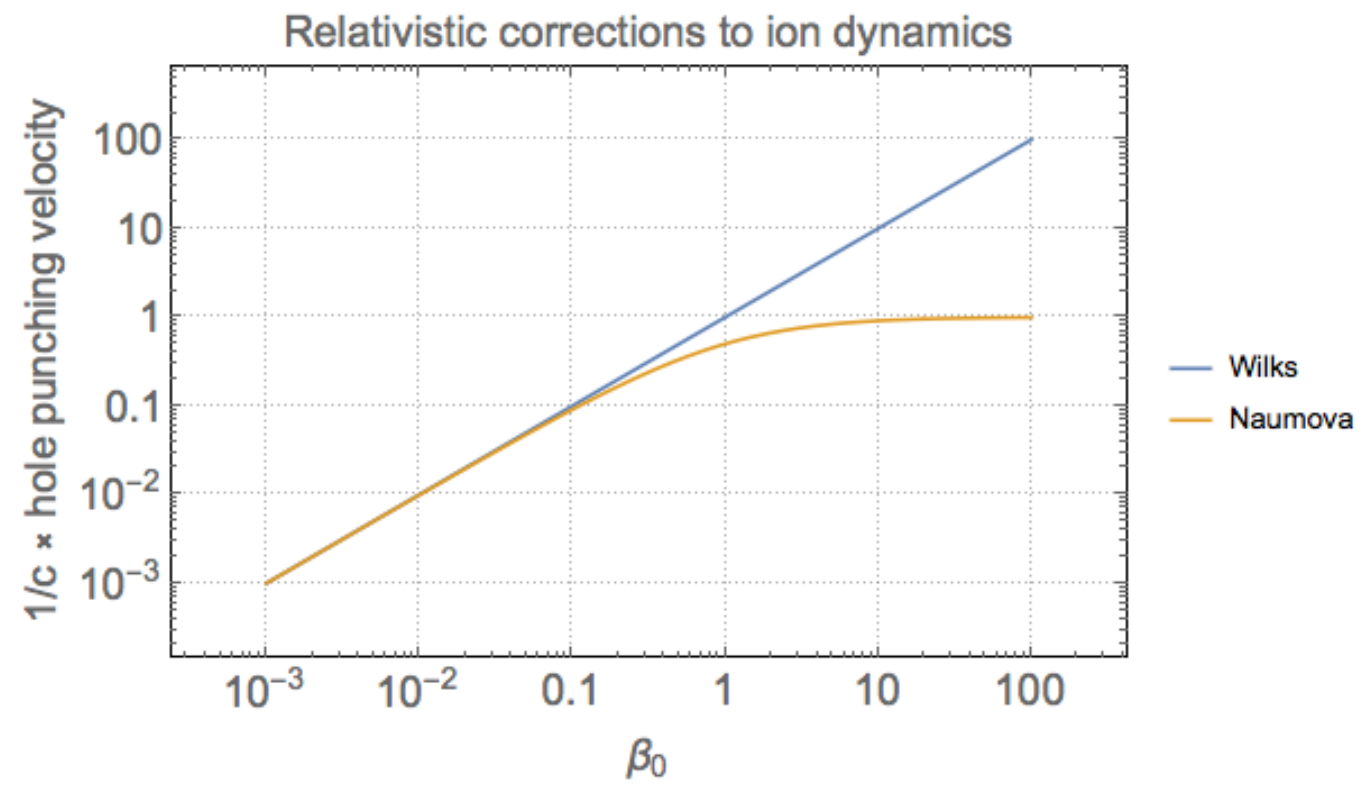

Figure 1.14 : Threshold for the onset of relativistic ion acceleration effects (see text).

\subsubsection{Relativistic electron beam generation}

The dynamics of petawatt laser acceleration of relativistic electrons is shown in the phase space in Fig. 1.12 (c). In this figure label (iii) shows electrons undergoing acceleration into the dense target in bunches, exhibiting a periodicity of twice the laser frequency, consistent with equation (1.7). The $2 \omega_{l}$ signature has been identified as a feature of multiple electron acceleration models $[95,84,85,140]$ and the magnitude of $p_{e} /\left(m_{e} c\right)$ is typically $\sim a_{0}$. As these hot electrons propagate into the target they heat the bulk plasma through Ohmic processes[87], causing the increased spread in the electron velocity shown by label (i). A much smaller fraction of the energetic electrons propagate backwards into the laser field, shown by label (ii). At the rear of the target, we see in label (iv) that electrons that have propagated through the target are trapped by a space-charge potential of the target, causing them to reflux with $p_{z}<0$ towards the laser. This space-charge potential is set up as the most energetic 
electrons escape in $\hat{z}$, accelerating ions from the target-rear with them, carrying out the TNSA ion acceleration process discussed in section 1.1.3. The signature of TNSA ions accelerated in this manner is evident in panel (d) label (ii) in the ion phase space. In this section we focus on the process of acceleration of relativistic electrons by the laser, i.e. the absorption of laser light and its conversion to electron kinetic energy. It is clear from the preceding description and Fig. 1.12 that this process is the driver of much of the system's physical richness.

A crucial question for the success of fast ignition ICF and many other petawatt laser applications relates to the energy of the relativistic electron beam. Since in realistic laser-plasma situations it is anticipated that multiple absorption processes will be acting concurrently, it is desirable to calculate the electron energy in a manner that is insensitive to the underlying mechanism of generation. The most widely used estimate for the electron energy is the ponderomotive scaling developed by Wilks et al. [215] as,

$$
T_{p}=\left(\gamma_{t}-1\right) m_{e} c^{2}
$$

where $\gamma_{t}=\sqrt{1+a_{0}^{2}}$ is the Lorentz factor accounting for transverse electron motion alone. Equation (1.15) was deduced from pioneering laser-plasma kinetic simulations in 1992 and to the present continues successful application. The ponderomotive scaling of the effective hot electron temperature generally reproduces most simulation and experimental data in typical conditions, i.e., $\sim$ few ps laser duration, moderate laser-to-ASE contrast implying the presence of a pre-plasma density profile, across a wide range of laser intensities, $10^{18} \mathrm{~W} \mu \mathrm{m}^{2} \mathrm{~cm}^{-2}<I \lambda_{\mathrm{l}}^{2}<5 \times 10^{21} \mathrm{~W} \mu \mathrm{m}^{2} \mathrm{~cm}^{-2}$ $[163,85,83,29,91]$.

Alternative models for the hot electron temperature have also been put forward[72, 


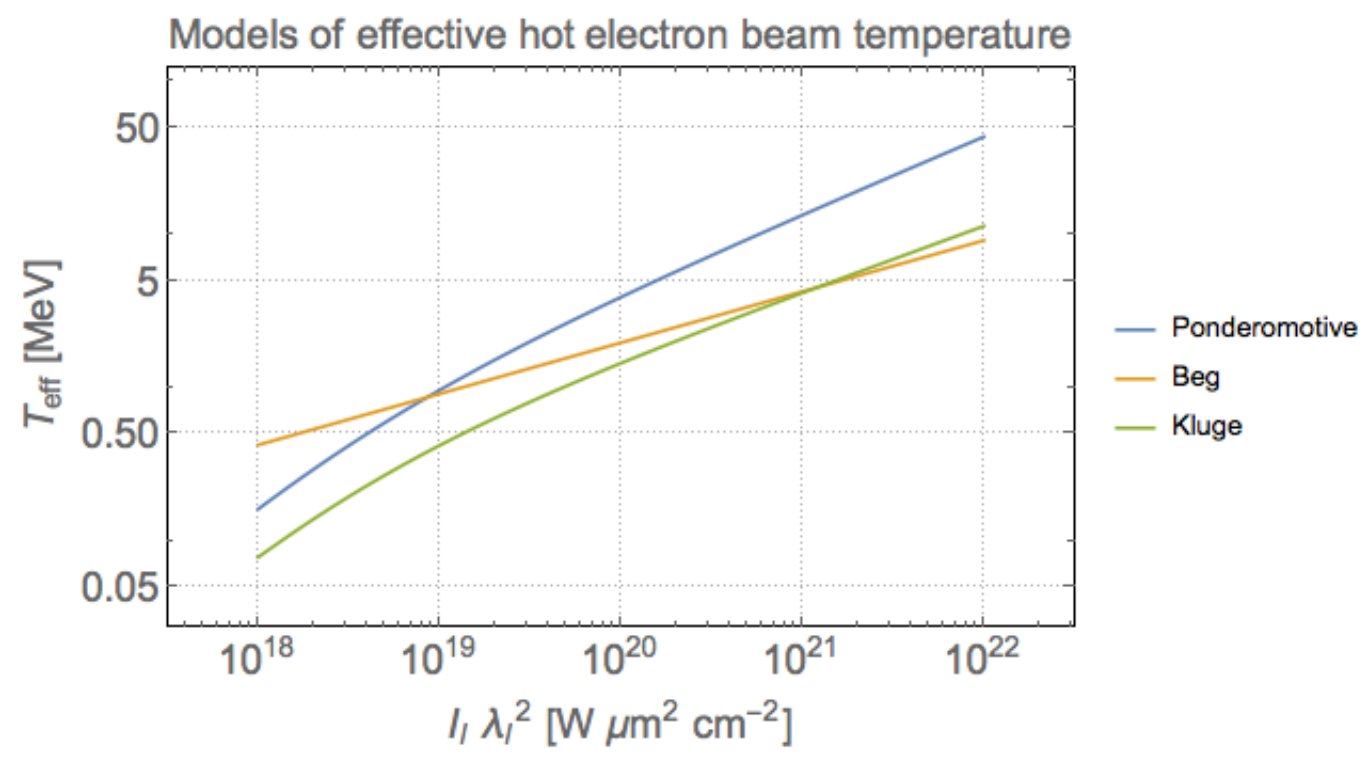

Figure 1.15: Comparison between models of the effective temperature of the relativistic electron beam (see text).

6, 92, 106, 145, 128]. An important empirical scaling found by Beg et al.[6] estimates that,

$$
T_{\text {beg }}=0.47 a_{0}^{2 / 3} \mathrm{MeV}
$$

implying a much cooler electron spectrum. It has been suggested that the discrepancy between the two models relates to the uncertainty in the actual on-target laser intensity, or to the laser-to-ASE contrast level, i.e., the degree of pre-plasma present $[100,134]$.

Another useful model of the hot electron temperature was developed by Kluge et al.[92], representing the electron distribution function with a general Lorentz-invariant 
ansatz. This work calculated the temperature to be,

$$
\langle\gamma\rangle_{\text {Kluge }}=\frac{4 \pi^{2}}{F\left(4 \pi^{2},-a_{0}^{2}\right)}
$$

where $F(a, b)$ denotes an elliptic integral of the second kind. The mathematics involved in its derivation imply that this temperature should be insensitive to the precise mechanism of electron acceleration. The effective electron temperature associated this model is then given by $T_{\text {Kluge }}=\left(\langle\gamma\rangle_{\text {Kluge }}-1\right) m_{e} c^{2}$. This expression is equivalent to equation (9) in ref. [92] for the hot electron temperature. The effective hot electron temperature predictions for the models discussed here are shown in Fig. 1.15. Detailed discussions and extensions of these calculations are presented in section 3.4 .

In this section we have presented an overview of existing theories of relativistic laser-plasma physics envisaged through the prism of the laser nonlinear force. The steady-state analysis in section 1.2.2 is extended in work presented in chapters 3 and 4 of this work. The relativistic electron beam generation analysis in section 1.2.3, related to the oscillatory component of the laser fields, has been developed in a manner that is insensitive to the precise mechanism of coupling. Aspects of this approach are discussed in more detail and extended in chapter 4 of this document.

\subsubsection{Quantum electrodynamical effects at ultra-relativistic intensity}

At the 10-petawatt-scale, laser absorption processes exhibit substantial feedback between electron-positron pair production, gamma-ray production and classical plasma physics.[170] As a consequence, this interaction regime has been termed with some controversy the 'QED-plasma' regime.[171] The basis of the transition from petawattscale interactions to quantum electrodynamical 10-petawatt-scale interactions relates 
to electronic radiation reaction effects. The energetics of an electron oscillating in the light fields imply the laser intensity at which radiative friction becomes important. To wit consider an electron having total relativistic energy $\mathcal{E}_{e}=\gamma_{e} m_{e} c^{2}$. The characteristic timescale $\tau_{c}$ over which the electron 'cools' by emission of radiation is,

$$
\tau_{c}=\frac{\gamma_{e} m_{e} c^{2}}{P_{\mathrm{rad}}} \simeq \frac{\ell_{c}}{c}
$$

where $\ell_{c}$ is the characteristic cooling length for the highly relativistic electrons, and the power radiated $d \mathcal{E}_{e} / d t$ is,

$$
P_{\mathrm{rad}}=\frac{a_{0}^{4} e^{2} \omega_{l}^{2}}{6 \pi c \epsilon_{0}}
$$

as given in ref. [47]. We consider electron motion within the axial length interval $x \in\left[0, \ell_{s}\right]$, with vacuum region $x<0$, corresponding to relativistic skin depth of the plasma,

$$
\ell_{s}=\frac{c \sqrt{\gamma_{e}}}{\omega_{\mathrm{pe}}}
$$

where the $\sqrt{\gamma_{e}}$ factor exhibits the relativistic mass increase of plasma electrons. This condition is relevant to relativistic laser interactions with solids presaging the calculations shown in chapter 3. In this region the (dimensionless) laser electric field is screened such that,

$$
a_{0}(x)=a_{0} e^{-\frac{x}{\ell_{s}}}
$$

Assuming that the average electron energy follows the ponderomotive scaling of 


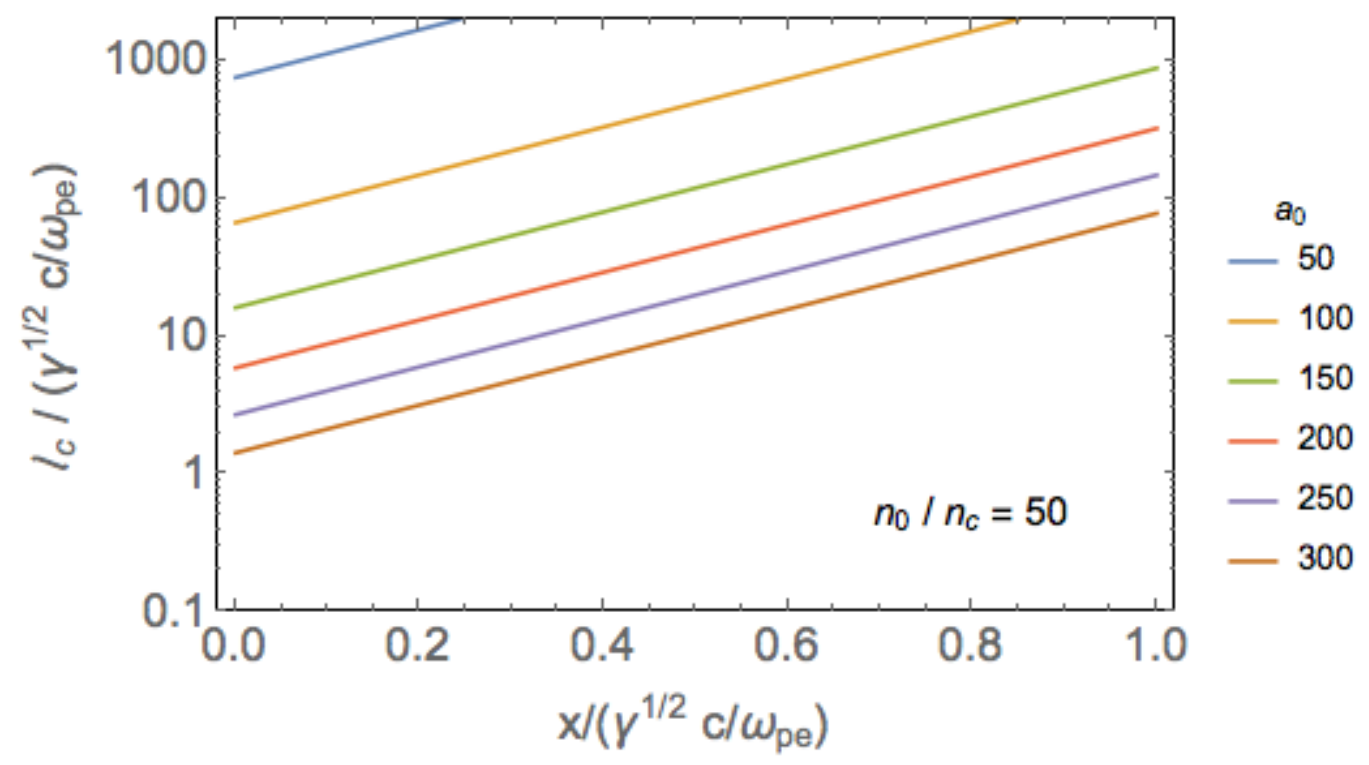

Figure 1.16 : Effects of radiative friction on relativistic electron motion in the plasma skin depth as a function of laser dimensionless field strength $a_{0}$. That the cooling length $\ell_{c}$ exceeds the skin depth $\ell_{s}$ while $a_{0}<300$ confirms that QED effects must be taken into account for 10-petawatt scale laser-plasma interactions.

Wilks[215], i.e., $\gamma_{e} \simeq a_{0}$, the electron cooling length is given by,

$$
\ell_{c}=\frac{6 \pi c^{4} m \epsilon_{0}}{a_{0}^{3} e^{2} \omega_{l}^{2}} e^{\frac{4 x \omega_{\mathrm{pe}}}{\sqrt{a_{0}} c}}=\frac{1.51 \times 10^{7}}{a_{0}^{3}} e^{\frac{4 x \omega_{\mathrm{p}}}{\sqrt{a_{0}} c}} \mu \mathrm{m}
$$

It is thus clear that QED effects will become important when the parameter[109],

$$
\frac{\ell_{c}}{\ell_{s}}=2.85 \times 10^{-3} a_{0}^{-7 / 2} e^{4 x / \ell_{s}} \sqrt{n_{0}\left[\mathrm{~cm}^{3}\right]}
$$

is of order unity. Fig. 1.16 shows curves corresponding to equation (1.23) for a $n_{0} / n_{c}=50$ plasma and a variety of laser dimensionless strength parameters. In this plot $\ell_{c} / \ell_{s} \sim 1$ when $a_{0} \sim 300$, indicating that QED effects should be taken into account for 10-petawatt-scale laser-plasma interactions, due to sheath effects in high 


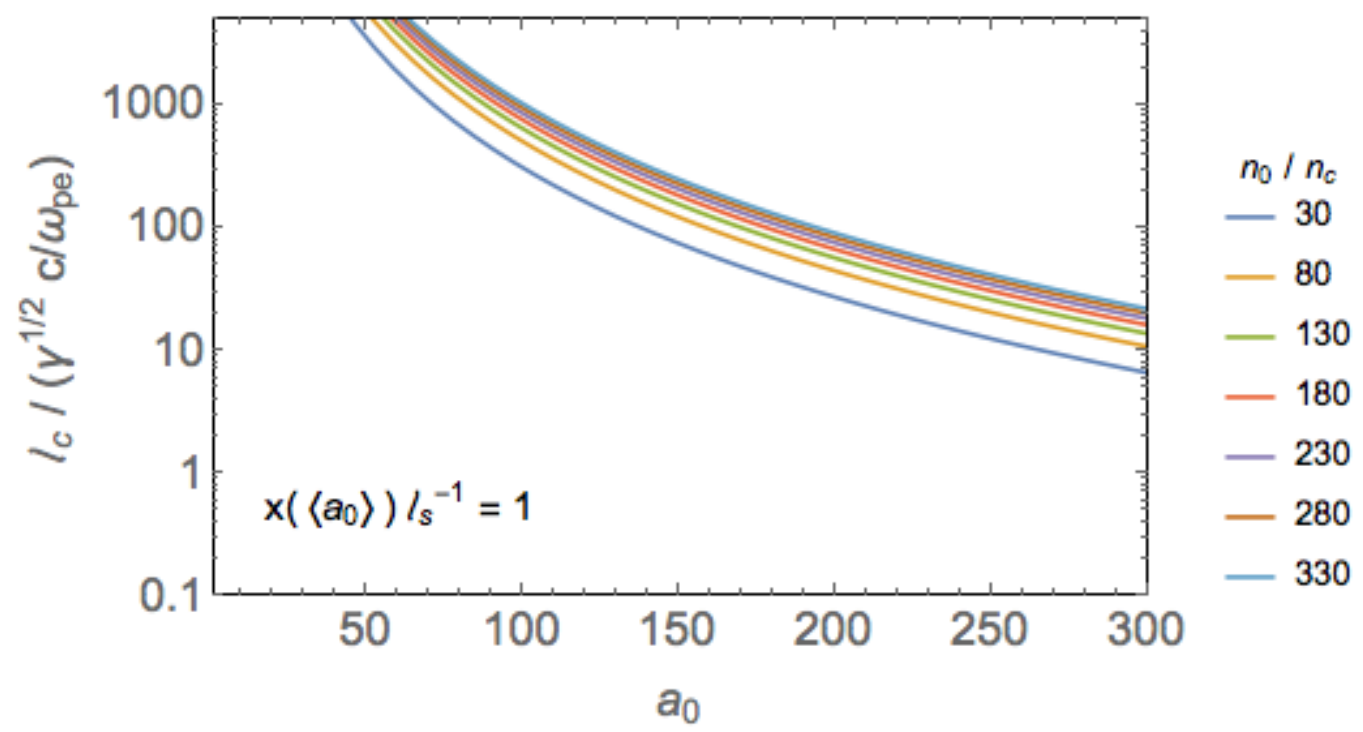

Figure 1.17 : Threshold for the onset of QED effects in petawatt laser-solid interactions as a function of laser dimensionless field strength $a_{0}$ for a variety of plasma densities, using equations (1.23) and (1.24).

density plasmas.

It is further instructive to elucidate the density dependence of radiative friction effects. Since $\ell_{s} / \lambda_{1} \ll 1$ the laser strength parameter in equation (1.21) can be approximated as,

$$
\left\langle a_{0}\right\rangle=\ell_{s}^{-1} \int_{0}^{\ell_{s}} a_{0} e^{-\frac{x}{\ell_{s}}} d x=\frac{(e-1) a_{0}}{e}
$$

Equation (1.24) shows that effective laser field over the skin depth occurs at $x\left(\left\langle a_{0}\right\rangle\right) / \ell_{s}=$ 0.46. Fig. 1.17 illustrates the predictions of equation (1.23) using this result as a function of $a_{0}$ for a variety of plasma densities. These results confirm that radiation reaction effects can be neglected for petawatt-scale laser interactions with solid targets, and illuminate a path towards understanding absorption at the 10-petawatt 
scale.

\subsection{Thesis structure overview}

This thesis unfolds itself in the following manner. In chapter 2 the primary numerical simulation method used to develop results, the particle-in-cell (PIC) approach, is described in detail. Chapter 3 covers the bulk of the research results derived over the course of the past years on the absorption of petawatt laser light by optically-thick plasmas. The relevant first-author publications for this section are refs. [106, 107, 108, 109]. Chapter 4 extends these results to include effects related to heterogeneous plasmas, including relativistically-underdense plasmas relevant to 'pre-plasma' situations, and realistic laser spatio-temporal profiles. This section is based on the first-author work in ref. [110], as well as presently unpublished work under document no. LLNL-JRNL-594672. Chapter 5 covers first-author work describing a mechanism of focusing high power laser light to higher intensities, in a fashion that could support reaching the QED-plasma regime, published in ref. [104]. In chapter 6 the development of a new simulation tool for understanding high energy density plasmas, most importantly those created using high energy and high power lasers through the processes described in preceding sections, is detailed. This section is based on first-author work in [105]. Finally in chapter 7 concluding remarks and a discussion of future research efforts is presented. 


\section{Chapter 2}

\section{Description of particle-in-cell simulation methods}

For the virtues of theoretical treatments, the nonlinear and kinetic character of relativistic laser-plasmas implies that utility should derive from numerical simulations. With this insight John Dawson developed the particle-in-cell (PIC) computational scheme in the 1960s[38], and research efforts over the intervening decades have realized some of this promise, delivering practitioners of the scheme considerable successes. Indeed, the discoveries of plasma-based accelerators of particles[205] and of photons[213] count among the breakthrough research enabled by PIC codes. Continuing to the present PIC codes play a fundamental role across many branches of plasma physics, including inertial confinement fusion research[204], magnetic fusion energy[51], laboratory astrophysics studies of the Weibel filamentation instability[209, 97, 77] and protogalactic seed magnetic field generation[69], large-scale international laser wakefield accelerator collaborations[103, 102], and many others. The application of PIC codes to the research covered in this document also bears fruit, supporting the elucidation and validation of the theoretical processes presented in chapters 3-5. So that the reader may usefully evaluate these efforts this chapter reviews the algorithms comprising the PIC simulation method. To this end the general workings of the PIC scheme are described in section 2.1, followed by descriptions of specific numerical schemes used by the hybrid fluid-PIC code LSP[211] in section 2.2, the code that is widely used to generate results presented in this thesis. 


\subsection{Description of the particle-in-cell scheme}

At their core PIC codes are designed to predict the time history of a system comprised of many charged particles. PIC codes solve this problem very generally, making few assumptions about the properties of the charged particles in the system. This generality implies the value of PIC codes as well as their computational expense.

PIC codes implement the Maxwell-Vlasov system of equations describing this general approach. Maxwell's equations cover the fields, currents, and so on that are associated with the moving charged particles comprising the system. The Vlasov equation describes the evolution of these particles in six dimensional phase space, i.e., three spatial coordinates and three momentum coordinates, and in time.

From this description we can discern the basic computational expense. Consider a petawatt laser-plasma situation relevant to section 1.2.1 having reduced electron density $n_{0} \sim 10 n_{c r}$ and covering a small cubic region of $\sim 10 \mu m$ on each side. Now imagine we are interested in simulating how the charges comprising this system react as a $\lambda_{1}=1 \mu m$ laser illuminates the front of this cube, over $\tau$ units of time (for instance, in $\left.\lambda_{\mathrm{l}} / c\right)$. If we estimate conservatively that 1 byte of computational memory is required to represent each phase space coordinate, for each particle in the system, then the total memory needed in $1 \tau$ is $120 \mathrm{~TB}\left(1 \mathrm{~TB}=10^{12}\right.$ bytes). Supercomputerscale resources would be required simply to represent the system in memory, and further costs accrue in relation to field and particle dynamics, so clearly numerical approximations are necessary.

PIC introduces two such techniques to reduce the memory requirements and enhance performance: it represents groups of identical particles as 'macroparticles' and discretizes space into a 'grid.' The electromagnetic fields in the simulation are computed at the grid vertices, or simply 'on the grid,' and interpolated back onto the 


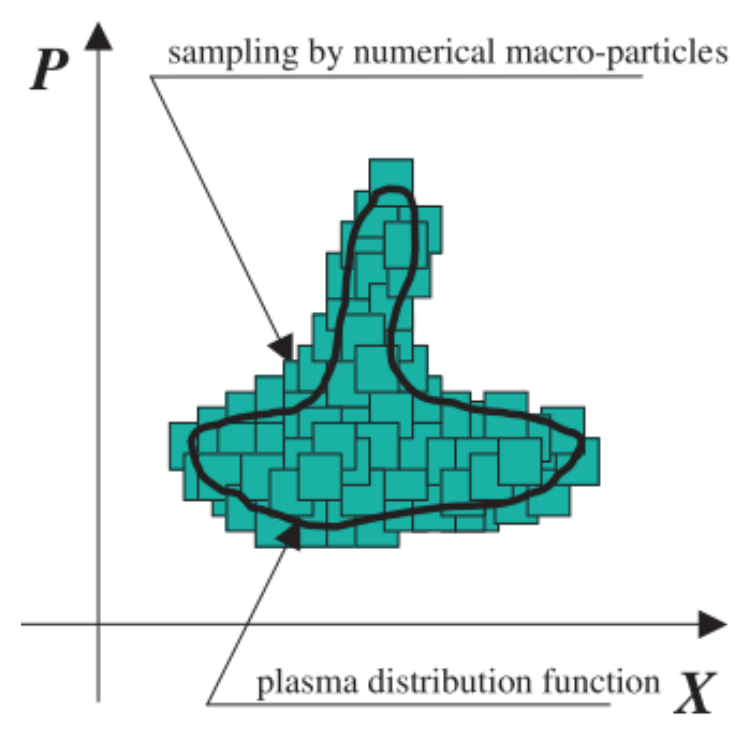

Figure 2.1 : Schematic showing the use of macroparticles in the PIC scheme to represent groups of identical particles, from ref. [166]. Collectively the macroparticles fully sample the phase space distribution of the system.

macroparticles existing within the grid 'cells.' Each macroparticle maintains the same charge-to-mass ratio of the particle it represents and so responds identically to Lorentz forces. Hence, the system's dynamics should be identical to the real situation and little generality is lost in terms of systems that may be represented. At the same time this approximation vastly reduces the number of objects stored in memory, reducing the computational burden. Macroparticles can be thought of as suitably sampling the distribution function of charges comprising the system, typically a plasma, as shown in Fig. 2.1.

The macroparticle approximation is not without drawbacks: since these objects have a finite (larger) size and finite (lower) number than the real particles they represent, their use introduces both artificial self-forces and statistical noise. The former effect is related to the process of adding macroparticles to the simulation grid, or 'casting to the grid.' This process depends on the macroparticle shape and is imple- 
mented in different fashions between PIC codes. If a particle's shape is point-like, since the fields within a given cell are interpolated from the cell onto the grid and vice versa, particles can exert spurious self-electrostatic forces.[11] Hence, the shape of a macroparticle is finite and often spread over more than one cell. In LSP the cloud-in-cell method can be used in order to enlarge the shape such that macroparticles span several cells, reducing the artificial self-forces[211]. The latter effect of statistical noise generally arises when the number of macroparticles used in the simulation is inadequate to resolve the plasma distribution function. These issues must be mitigated through careful understanding and calibration of the simulations, e.g., increasing the number of macroparticles in the simulation through many tests and iterations until the system's dynamics are observed to converge.

Even with a suitable treatment of the macroparticles issues arise due to the discretized nature of the spatial grid. The size of the cells comprising the grid must be chosen in order to resolve the relevant physical scales in the situation of interest. For instance, accurately simulating the laser interaction with the $(10 \mu m)^{3}$ cubic plasma discussed above means setting the grid cell size $\Delta x$ equal to plasma skin depth $\sim \ell_{p} / 2 \simeq 0.027 \mu m$. The number of uniform cells required in this simulation is therefore $(10 \mu m / 0.027 \mu m)^{3} \sim 5 \times 10^{7}$. While computationally expensive such three dimensional PIC simulations are performed with some frequency using massively-parallel supercomputing clusters. Due to this expense two dimensional PIC simulations, which here would require only $\sim 10^{5}$ cells, are of more common usage.

The increment by which PIC simulations are advanced in time, the 'timestep' $\Delta t$, is also typically determined by the grid resolution $\Delta x$. Since we are interested in laser interactions causality requires that $\Delta t \leq \Delta x / c$, related to the Courant condition for numerical stability[11, 75]. For a typical petawatt laser-dense plasma situation 
$\Delta t \sim 0.1$ fs or smaller. Over many hundreds, thousands or millions of $\Delta t$, PIC codes evolve the Maxwell-Vlasov equations on the $\Delta x$ resolution grid. By this process PIC codes obtain the time history of the system of charged particles.

It is instructive to consider the analytic and numeric implementations that allow these equations to be solved in a self-consistent manner over time. The essential PIC methods are represented in the 'explicit' numerical solution algorithm, which is implemented in codes such as ZOHAR[11], PSC[185], PICLS[196] and many others. An illustration of the numerical advancement procedures associated with the particles and fields in the explicit scheme is shown below in Fig. 2.2. In examining its workings we follow ref. [183] and use standard SI units. The Vlasov equation describing the particle phase space evolution is given by,

$$
\begin{aligned}
& q_{k} \frac{\partial f_{k}}{\partial \vec{p}_{k}}\left(\vec{v}_{k} \times \vec{B}+\vec{E}\right)+\vec{v}_{k} \frac{\partial f_{k}}{\partial \vec{x}}+\frac{\partial f_{k}}{\partial t}= \\
& \Sigma_{\mathrm{l}=\mathrm{n}, \mathrm{e}, \mathrm{i}} \int d^{3} p_{l} v_{\mathrm{kl}} \int d \Omega_{\psi} \sigma^{\mathrm{kl}}\left(f_{k}^{\prime} f_{l}^{\prime}-f_{k} f_{l}\right)
\end{aligned}
$$

where $f_{k}(\vec{x}, \vec{p}, t)$ is the distribution function of the $k^{t h}$ particle species describing the probability of existing in a given region of phase space. In other words, $f_{k}(\vec{x}, \vec{p}, t)=$ $\sum_{n}^{N_{k}} \delta\left(\vec{x}-\vec{x}_{n}(t)\right) \delta\left(\vec{p}-\vec{p}_{n}(t)\right)$ summed over the $N_{k}$ macroparticles comprising species $k$. Here $m_{k}$ is the mass, $q_{k}$ is the charge, $\vec{p}_{k}$ is the momentum, $\vec{v}_{k}$ is the velocity and $\vec{x}_{k}$ is the position. The right hand side of equation (2.1) describes particle collisionality and is summed over the electron, ion and neutral particle species. Hence, equation (2.1) concisely represents $\sum_{k} N_{k}$ phase space density conservation equations for each of the macroparticles in the simulation; in practice, it is typically discretized as $\sum_{k} N_{k}$ equations for numerical efficiency. The collision cross section is $\sigma^{\mathrm{kl}}$ and $\mathrm{d} \Omega_{\psi}=$ $\mathrm{d} \nu \mathrm{d} \psi \sin \psi$ is the solid angle element of angles $\psi$ and $\nu$ between $p_{k}$ and $p_{k}^{\prime}$ describing the three dimensional particle scattering, the latter being the variable of integration. 
The relative velocity between particles $k$ and $l$ can be expressed as,

$$
v_{\mathrm{kl}}=\frac{c F_{\mathrm{kl}}}{p_{k}^{0} p_{l}^{0}}=\sqrt{\left(\vec{v}_{k}-\vec{v}_{l}\right)^{2}-\frac{\left(\vec{v}_{k} \times \vec{v}_{l}\right)^{2}}{c^{2}}}
$$

Equation (2.1) together with Maxwell's equations,

$$
\begin{aligned}
& \frac{\partial \vec{E}}{\partial t}=c^{2} \vec{\nabla} \times \vec{B}-\frac{\vec{J}}{\epsilon_{0}} \\
& \frac{\partial \vec{B}}{\partial t}=-\vec{\nabla} \times \vec{E} \\
& \frac{\partial \rho}{\partial t}=-\vec{\nabla} \cdot \vec{J}
\end{aligned}
$$

where the charge and current densities are defined as,

$$
\begin{aligned}
\rho & =q_{e} \int d^{3} p_{e} f_{e}+q_{i} \int d^{3} p_{i} f_{i} \\
\vec{J} & =q_{e} \int d^{3} p_{e} \vec{v}_{e} f_{e}+q_{i} \int d^{3} p_{i} \vec{v}_{i} f_{i}
\end{aligned}
$$

form the complete description of the Maxwell-Vlasov system.

In order to facilitate the discretization of equations (2.1-2.5) we employ the dimensionless scalings,

$$
\begin{array}{r}
t \rightarrow t \omega_{l}, \quad \vec{x} \rightarrow \frac{\vec{x} \omega_{l}}{c}, \quad \vec{p}_{k} \rightarrow \frac{\vec{p}_{k}}{c m_{k}} \\
\vec{E} \rightarrow \frac{\vec{E}}{E_{0}}, \quad \vec{B} \rightarrow \frac{\vec{B}}{B_{0}} \\
\vec{J} \rightarrow \frac{\vec{J}}{J_{0}}, \quad \rho \rightarrow \frac{\rho}{\rho_{0}} \\
B_{0}=\frac{E_{0}}{c}, \quad j_{0}=E_{0} \epsilon_{0} \omega_{l}, \quad \rho_{0}=B_{0} \epsilon_{0} \omega_{l}
\end{array}
$$

where by the field's convention we have introduced parameters associated with the 
laser as references: $\omega_{l}$ is the laser angular frequency and $E_{0}$ is the initial laser electric field given in relation to $a_{0}$ by equation (1.2). Since in section 1.2.1 it was shown that petawatt laser-plasma situations are typically collisionless in the regimes of interest we proceed in the limit that $\sigma^{k l} \rightarrow 0$. In the normalized units of equation (2.8) the electric field equations are given by,

$$
\begin{aligned}
\frac{\partial E_{x}}{\partial t} & =-\frac{\partial B_{y}}{\partial z}+\frac{\partial B_{z}}{\partial y}-J_{x} \\
\frac{\partial E_{y}}{\partial t} & =\frac{\partial B_{x}}{\partial z}-\frac{\partial B_{z}}{\partial x}-J_{y} \\
\frac{\partial E_{z}}{\partial t} & =-\frac{\partial B_{x}}{\partial y}+\frac{\partial B_{y}}{\partial x}-J_{z}
\end{aligned}
$$

The magnetic field equations are,

$$
\begin{aligned}
\frac{\partial B_{x}}{\partial t} & =\frac{\partial E_{y}}{\partial z}-\frac{\partial E_{z}}{\partial y} \\
\frac{\partial B_{y}}{\partial t} & =\frac{\partial E_{z}}{\partial x}-\frac{\partial E_{x}}{\partial z} \\
\frac{\partial B_{z}}{\partial t} & =\frac{\partial E_{x}}{\partial y}-\frac{\partial E_{y}}{\partial x}
\end{aligned}
$$

and the charge density evolves according to,

$$
\frac{\partial \rho}{\partial t}=-\frac{\partial J_{x}}{\partial x}-\frac{\partial J_{y}}{\partial y}-\frac{\partial J_{z}}{\partial z}
$$

These equations are discretized by the finite-difference time domain (FDTD) method using the variable $F_{\mathrm{jkl}}^{n}=F(j \Delta \mathrm{x}, k \Delta \mathrm{y}, l \Delta \mathrm{z}, n \Delta \mathrm{t})$ containing the spatial derivatives of the electric and magnetic fields. Here $j, k, l, n$ are the step sizes along their respective 


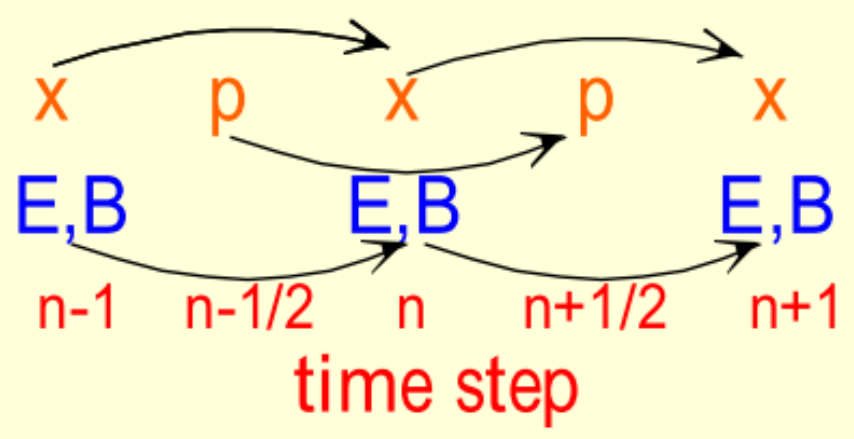

Figure 2.2 : Schematic illustrating the 'leapfrog' time stepping sequence associated with the explicit solution method, from ref. [210]. This figure shows that the Vlasov equation governing the particle phase space evolution $(x, p)$ and Maxwell's equations governing the electromagnetic field evolution $E, B$ are advanced in an alternating fashion.

coordinate directions. The discretized spatial derivatives are then written as,

$$
\begin{aligned}
\vec{\nabla}^{-} F_{\mathrm{jkl}}^{n} & =\left(\frac{F_{\mathrm{jkl}}^{n}-F_{j-1 \mathrm{kl}}^{n}}{\Delta \mathrm{x}}, \frac{F_{\mathrm{jkl}}^{n}-F_{\mathrm{jk}-1 l}^{n}}{\Delta \mathrm{y}}, \frac{F_{\mathrm{jkl}}^{n}-F_{\mathrm{jkl}-1}^{n}}{\Delta \mathrm{z}}\right) \\
\vec{\nabla}^{+} F_{\mathrm{jkl}}^{n} & =\left(\frac{F_{j+1 \mathrm{kl}}^{n}-F_{\mathrm{jkl}}^{n}}{\Delta \mathrm{x}}, \frac{F_{\mathrm{jk}+1 l}^{n}-F_{\mathrm{jkl}}^{n}}{\Delta \mathrm{y}}, \frac{F_{\mathrm{jkl}+1}^{n}-F_{\mathrm{jkl}}^{n}}{\Delta \mathrm{z}}\right)
\end{aligned}
$$

where directionality is indicated by the sign superscript associated with $\vec{\nabla}$. Equations (2.16-2.17) highlight the fact that the electromagnetic fields are advanced in full integer units of $n$, consistent with Fig. 2.2. $F_{\mathrm{jkl}}^{n}$ satisfies the conditions that,

$$
\vec{\nabla}^{-} \cdot \vec{\nabla}^{-} \times F_{\mathrm{jkl}}^{n}=0, \quad \vec{\nabla}^{+} \cdot \vec{\nabla}^{+} \times F_{\mathrm{jkl}}^{n}=0
$$


With this the discretized versions of Maxwell's equations can now be written as,

$$
\begin{aligned}
\frac{\vec{E}_{\mathrm{jkl}}^{\mathrm{n}+1 / 2}-\vec{E}_{\mathrm{jkl}}^{\mathrm{n}-1 / 2}}{\Delta \mathrm{t}} & =\vec{\nabla}^{-} \times \vec{B}_{\mathrm{jkl}}^{\mathrm{n}}-\vec{J}_{\mathrm{jkl}}^{\mathrm{n}} \\
\frac{\vec{B}_{\mathrm{jkl}}^{\mathrm{n}+1}-\vec{B}_{\mathrm{jkl}}^{\mathrm{n}}}{\Delta \mathrm{t}} & =-\vec{\nabla}^{+} \times \vec{E}_{\mathrm{jkl}}^{\mathrm{n}+1 / 2} \\
\frac{\rho_{\mathrm{jkl}}^{\mathrm{n}+3 / 2}-\rho_{\mathrm{jkl}}^{\mathrm{n}+1 / 2}}{\Delta \mathrm{t}} & =-\vec{\nabla}^{-} \cdot \vec{J}_{\mathrm{jkl}}^{\mathrm{n}+1}
\end{aligned}
$$

where

$$
\begin{aligned}
\vec{E}_{\mathrm{jkl}}^{\mathrm{n}+1 / 2} & =\left(E_{x \mathrm{j}+1 / 2 \mathrm{kl}}^{\mathrm{n}+1 / 2}, E_{y \mathrm{jk}+1 / 2 \mathrm{l}}^{\mathrm{n}+1 / 2}, E_{z \mathrm{jkl}+1 / 2}^{\mathrm{n}+1 / 2}\right) \\
\vec{B}_{\mathrm{jkl}}^{\mathrm{n}} & =\left(B_{x \mathrm{jk}+1 / 2 \mathrm{l}+1 / 2}^{\mathrm{n}}, B_{y j \mathrm{j}+1 / 2 \mathrm{kl}+1 / 2}^{\mathrm{n}}, B_{z \mathrm{j}+1 / 2 \mathrm{k}+1 / 2 \mathrm{l}}^{\mathrm{n}}\right) \\
\vec{J}_{\mathrm{jkl}}^{\mathrm{n}+1} & =\left(J_{x \mathrm{j}+1 / 2 \mathrm{kl}}^{\mathrm{n}+1}, J_{y j \mathrm{jk}+1 / 21}^{\mathrm{n}+1}, J_{z \mathrm{jkl}+1 / 2}^{\mathrm{n}+1}\right)
\end{aligned}
$$

The half-integer $n$ values appearing in equations (2.19-2.24) presage the role of the Vlasov solver. Indeed the particle evolution occurs according to the discretized equations,

$$
\begin{aligned}
\vec{E}_{\mathrm{jkl}}^{\mathrm{n}+1 / 2}-\vec{E}_{\mathrm{jkl}}^{\mathrm{n}} & =\frac{1}{2} \Delta \mathrm{t}\left(\vec{\nabla}^{-} \times \vec{B}_{\mathrm{jkl}}^{\mathrm{n}}-\vec{J}_{\mathrm{jkl}}^{\mathrm{n}}\right) \\
\vec{B}_{\mathrm{jkl}}^{\mathrm{n}+1 / 2}-\vec{B}_{\mathrm{jkl}}^{\mathrm{n}} & =-\frac{1}{2} \Delta \mathrm{t} \vec{\nabla}^{+} \times \vec{E}_{\mathrm{jkl}}^{\mathrm{n}+1 / 2} \\
\frac{\rho_{\mathrm{jkl}}^{\mathrm{n}+3 / 2}-\rho_{\mathrm{jkl}}^{\mathrm{n}+1 / 2}}{\Delta \mathrm{t}} & =-\vec{\nabla}^{-} \cdot \vec{J}_{\mathrm{jkl}}^{\mathrm{n}+1} \\
\vec{B}_{\mathrm{jkl}}^{\mathrm{n}+1}-\vec{B}_{\mathrm{jkl}}^{\mathrm{n}+1 / 2} & =-\frac{1}{2} \Delta \mathrm{t} \vec{\nabla}^{+} \times \vec{E}_{\mathrm{jkl}}^{\mathrm{n}+1 / 2} \\
\vec{E}_{\mathrm{jkl}}^{\mathrm{n}+1}-\vec{E}_{\mathrm{jkl}}^{\mathrm{n}+1 / 2} & =\frac{1}{2} \Delta \mathrm{t}\left(\vec{\nabla}^{-} \times \vec{B}_{\mathrm{jkl}}^{\mathrm{n}+1}-\vec{J}_{\mathrm{jkl}}^{\mathrm{n}+1}\right)
\end{aligned}
$$

Equations (2.19-2.29) fully specify the explicit solution algorithm, in concert with relevant initial and boundary conditions. This description outlines the linear inter- 
polation method relevant to the PSC[185] and LSP[211] numerical implementations. More sophisticated numerical integration algorithms for partial differential equations that capture quadratic and cubic curvature, through the finite difference scheme, are implemented in PIC codes such as OSIRIS[55].

The explicit scheme described in this section is the simplest implementation of PIC and therefore illustrates its core numerical workings. This method of solution is relevant to the PSC[185] code used to perform simulations supporting the research covered in chapter 5 of this document.

\subsection{Details of selected numerical schemes implemented in the LSP code}

The 'direct implicit' scheme is a more sophisticated method of solution. Its algorithms seek to predict future values associated with the particles and fields, a feature which relaxes constraints on the simulation timestep and helps improve numerical stability. In LSP's implementation the field solver is based on an unconditionally Courantstable numerical approach, enabling the use of simulation timesteps much larger than $\Delta x / u$, for simulation cell size $\Delta x$ and characteristic velocity scale $u(=c$ for laser interactions).[211] A schematic of the direct implicit algorithm illustrating its methods of solution is shown in Fig. 2.3.

This section summarizes the implementation of the direct implicit scheme of Friedman et al.[58], as outlined by Welch et al.[211] and implemented in the version of LSP used in this dissertation. The momentum $\vec{p}$ of each macroparticle is advanced according to,

$$
\vec{p}_{n+1 / 2}=\Delta \mathrm{t}\left[\left(\vec{p}_{n-1 / 2}+\vec{p}_{n+1 / 2}\right) \times \vec{B}_{n}\left(\vec{x}_{n}\right) \frac{q}{2 c m \gamma_{n}}+\vec{a}_{n}\right]+\vec{p}_{n-1 / 2}
$$




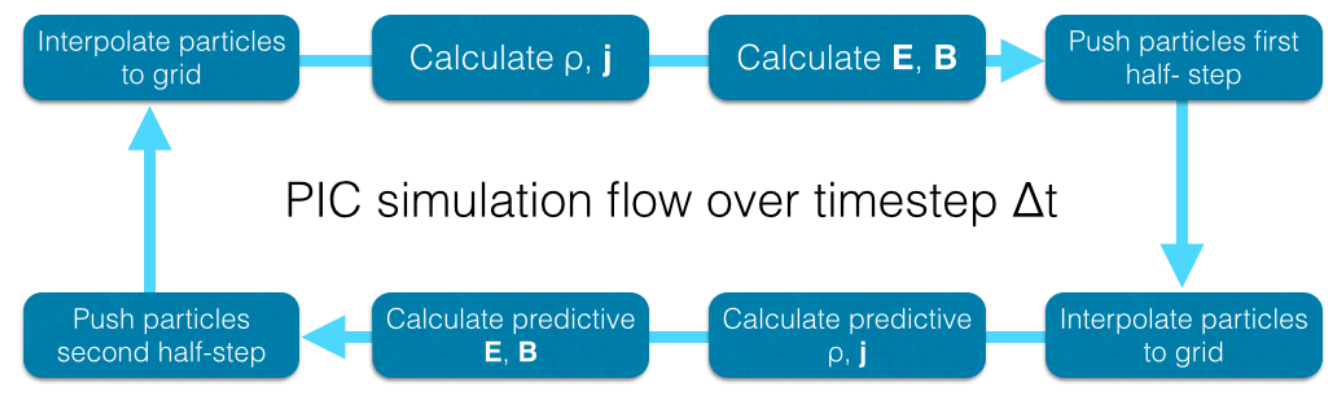

Figure 2.3 : Schematic illustrating the predictive time stepping sequence associated with the direct implicit solution method. This figure shows that due to the predictive nature of the algorithm particles are effectively advanced twice.

where $\gamma$ is the Lorentz factor and full-integer $n$ subscripts correspond to values at full timesteps. The electric field information is described by $\vec{a}_{n}$ as,

$$
\vec{a}_{n}=\frac{1}{2}\left(\vec{a}_{n-1 / 2}-\frac{q}{m} \vec{E}_{n+1 / 2}\left(\vec{x}_{n+1 / 2}\right)\right)
$$

thus containing information about the field at $n-1 / 2$ and $n+1 / 2$. Particles are pushed twice according to equations (2.30-2.31) with the initial $\vec{E}_{n+1 / 2}\left(\vec{x}_{n+1 / 2}\right) \rightarrow 0$. The first particle push is performed using $\vec{E}_{n+1}\left(\vec{x}_{n+1}\right)=0$. The fields are then updated using perturbative analysis, as described below, in order to predict the effect of $\vec{E}_{n+1}\left(\vec{x}_{n+1}\right)$ on the perturbed current. In the second push particle positions and momenta are updated using this correction. In order to facilitate numerical calculations, the particle momenta given by equation (2.30) can be expressed in terms of matrix operations as,

$$
\vec{p}_{n+1 / 2}=\langle T\rangle \cdot \vec{A}
$$


using the magnetic field rotation tensor $\langle\mathrm{T}\rangle$,

$$
\langle\mathrm{T}\rangle=\frac{1}{1+\Omega^{2}}\left(\begin{array}{ccc}
\Omega_{x}^{2}+1 & \Omega_{x} \Omega_{y}+\Omega_{z} & \Omega_{x} \Omega_{z}-\Omega_{y} \\
\Omega_{x} \Omega_{y}-\Omega_{z} & \Omega_{y}^{2}+1 & \Omega_{x}+\Omega_{y} \Omega_{z} \\
\Omega_{y}+\Omega_{x} \Omega_{z} & \Omega_{y} \Omega_{z}-\Omega_{x} & \Omega_{z}^{2}+1
\end{array}\right)
$$

associated with the three dimensional magnetic contribution to the Lorentz force, and,

$$
\vec{\Omega}=\frac{q \vec{B}_{n}}{2 c \gamma m} \Delta \mathrm{t}
$$

with $\Omega^{2}=|\vec{\Omega}|^{2}$. The source vector $\vec{A}$ related to equation (2.32) is given by,

$$
\vec{A}=\vec{p}_{n-1 / 2} \times \vec{\Omega}+\vec{a}_{n} \Delta \mathrm{t}+\vec{p}_{n-1 / 2}
$$

The corrections to the fields following the first push are calculated by perturbative analysis, as indicated above. Similarly corrections to the particle velocities are determined by,

$$
\vec{v}=\vec{v}^{1}+\delta \vec{v}
$$

where $\vec{v}^{1}$ is the velocity calculated from the first push. The correction to the Lorentz factor is given by $\delta \gamma=\vec{v} \cdot \delta \vec{E}\left[\delta \vec{E}=\vec{E}_{n+1 / 2}\left(\vec{x}_{n+1 / 2}\right)\right]$, which implies that,

$$
\delta \vec{v}=\frac{q \Delta \mathrm{t}}{m \gamma_{n+1 / 2}}\left(\langle\mathrm{~T}\rangle \cdot \delta \vec{E}-\vec{v}_{n+1 / 2}\left(\vec{v}_{n+1 / 2} \cdot \delta \vec{E}\right)\right)
$$

Equation (2.37) can now be used to determine corrections to the current density: $\vec{J}=$ $\vec{J}^{1}+\delta \vec{J}$ using $\delta \vec{J}=\rho \partial \vec{x} / \partial t$. This value can be written in terms of the susceptibility 
tensor $\langle\mathrm{S}\rangle$ as $\delta \vec{J}=\langle\mathrm{S}\rangle \cdot \delta \vec{E}$, where,

$$
\langle\mathrm{S}\rangle=\frac{\rho q \Delta \mathrm{t}}{2 m \gamma_{n+1 / 2}}\left(\langle T\rangle-\vec{v}_{n+1 / 2} \wedge \vec{v}_{n+1 / 2}\right)
$$

with $\wedge$ denoting the dyadic product. In the LSP implementation $\langle\mathrm{S}\rangle$ associated with each particle is summed after the first push such that charge is conserved. LSP's implementation also conserves energy in this process. This perturbed current $\delta \vec{J}$ is then used to correct the particle positions and momenta from the first particle push. Using equation (2.38) the electromagnetic field equations can be written as,

$$
\begin{aligned}
\frac{\partial \vec{E}}{\partial t} & =-\langle\mathrm{S}\rangle \cdot \delta \vec{E}-\vec{J}^{1}+\vec{\nabla} \times \vec{B} \\
\frac{\partial \vec{B}}{\partial t} & =-\vec{\nabla} \times \vec{E}
\end{aligned}
$$

where $\vec{J}^{1}$ is the current density from the first push. Finite differencing is then applied to these equations in a manner analogous to equations (2.19-2.29) in the preceding section.

The direct implicit solution method described in this section is implemented in the LSP code[211], which has been used to support the research described in chapters 3-4 of this document. 


\section{Chapter 3}

\section{Absorption of ultraintense laser light by solid matter}

\subsection{Conservation laws and conversion efficiency in ultrain- tense laser-overdense plasma interactions}

In this section we develop a set of mass, momentum and energy conservation laws applying to a broad range of relativistic laser interactions with optically-thick plas-

mas. Based on these conservation laws, we then derive an advanced fully-relativistic absorption model that yields formulae for key dynamical quantities of the interaction: the fraction of laser energy absorbed by the plasma, the precise sub-fractions of laser energy absorbed by ions and by electrons comprising the plasma, the ensemble average energies of these ions and electrons, and the velocity at which the laser advances ('punches') into the plasma.

In section 3.1.1 we describe the general scenario, geometry and electron and ion particle populations involved in the conservation laws. In section 3.1.2 we develop the relevant mass conservation laws and in section 3.1.3 we develop the relevant momentum and energy conservation laws. The absorption model is derived using these conservation laws in section 3.1.4. Here equations for the laser 'hole punching' velocity and the ensemble average ion and electron energies are also calculated. In section 3.1.5 we derive expressions for the absorption of laser energy and its subpartitioning into plasma electrons and ions. Finally, in section 3.1.6 we validate the predictions 
of the absorption model against particle-in-cell simulations: excellent agreement is demonstrated. We close this section with a discussion of possible applications of the model to astrophysically-relevant scenarios.

\subsubsection{Coupling of ultraintense laser light to an overdense plasma}

We consider the steady-state laser-plasma interaction at the coupling stage in the laboratory frame. Consider the small volume at the laser-plasma interface comprising a relativistic collisionless skin depth in a axial extent, depicted schematically in Fig. 3.1. The laser is incident on the volume from the left and excites plasma particles which leave the volume on the right-hand side. All particles in the region are assumed to interact with the laser and may be accelerated relativistically by coupling into either the oscillatory or steady-state absorption mode. The total electron density is $n_{e}$, which may take on any value that is relativistically-opaque to the laser light[26]. Ions in the interface are assumed to have uniform charge state $Z$ given by the quasineutrality condition, $n_{i}=n_{e} / Z$.

Absorption of ultraintense laser light by an overdense plasma amounts, in effect, to the coupling between an incident photon flux and particles comprising the moving plasma interface. It follows that we consider only plasma particles that mediate the energy and momentum exchange. That is, those particles that are directly excited, i.e. accelerated, by the laser on the spatial scale $c / \omega_{p e}$ at the laser-plasma (LP) interface. The set $s$ fully enumerates these populations,

$$
s \in\{\text { hp ions, hp electrons, hot electrons }\}
$$


$\mathrm{n}_{0}$

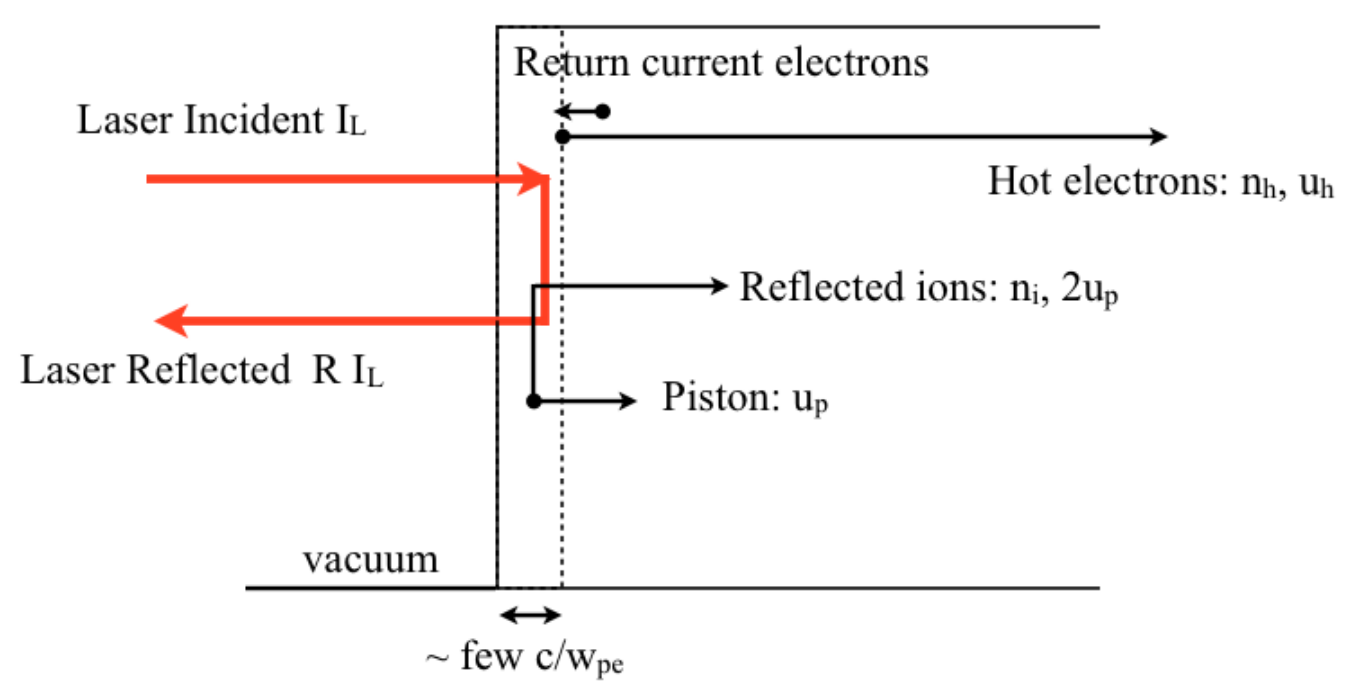

Figure 3.1 : Schematic depicting the laser plasma coupling in the interaction region with $n_{0}=n_{e}=Z n_{i}$ where $\mathrm{Z}$ is the ion charge state. The laser piston boundary is represented by the dashed line.

where in the following sections each species will be referred to by its numeric index. Once the laser has coupled into these populations, energy and momentum may then cascade into other species in the plasma. An important example of this is the collective plasma excitation of a return current in order to neutralize the fast electron current[72].

The evolution of particle distribution functions $f_{s}\left(x^{\mu}, p^{\mu}\right)$ in equation (3.1) is determined by the Boltzmann-Vlasov equation. Here $x^{\mu}=(c t, \mathbf{x})$ represents the four position coordinates and $p^{\mu}=\gamma m c(1, \mathbf{u} / c)$ represents the four momentum coordinates. The collisionless, manifestly covariant form of equation (2.1), convenient for our purposes here, can be written as,

$$
\left(\frac{d x^{\mu}}{d \tau} \frac{\partial}{\partial x^{\mu}}+\frac{d p^{k}}{d \tau} \frac{\partial}{\partial p^{k}}\right) f_{s}=0
$$


where $\tau$ is the proper time[185, 73], i.e., the temporal coordinate evaluated in the frame of reference in which particles are instantaneously at rest. Throughout this work we establish the conventions that the Minkowski tensor $\eta^{\mu \nu}$ has signature $(-,+,+,+)$; the greek sub- and super-scripts represent tensor indices $\mu \in\{0,1,2,3\}$; the latin indices $k \in\{1,2,3\}$ run over the spatial subset; the $s$ subscript is reserved for particle species and for clarity will be distinguished from tensor indices where necessary. Collisional coupling is assumed to be negligible and the characteristics of equation (3.2) are given by,

$$
\frac{d x^{\mu}}{d \tau}=\frac{p^{\mu}}{m_{s}}, \quad \frac{d p^{k}}{d \tau}=-\frac{q_{s}}{m_{s}} F^{k \mu} p_{\mu}
$$

where the momentum characteristic includes spatial components due to the massshell restriction[73]. In equations (3.2) and (3.3), $q_{s}$ is the electric charge, $m_{s}$ is the rest mass and $F^{\mu \nu}$ represents the field strength tensor,

$$
F^{\mu \nu}=\left(\begin{array}{cccc}
0 & E_{x} & E_{y} & E_{z} \\
-E_{x} & 0 & -B_{z} & B_{y} \\
-E_{y} & B_{z} & 0 & -B_{x} \\
-E_{z} & -B_{y} & B_{x} & 0
\end{array}\right)
$$

The approach undertaken in this section considers the parameters of $f_{s}$ that satisfy conservation of energy and momentum between the ultraintense photon flux and relativistic particle fluxes excited in the plasma, i.e., the moments of equation (3.2). At each transverse point along the laser-matter interface, $f_{s}=f_{s}\left(p^{\mu}\right)$. Thusly formulated the germane task is to determine the moments of $f_{s}$, i.e., $\int p^{\mu} f_{s} d p^{k}$ corresponding to the ensemble average Lorentz factor and velocities, for the hp particles and hot elec- 
trons such that energy and momentum are conserved between the plasma and the laser driver.

Before performing calcuations related to equation (3.2), a brief asside on the particle distribution functions describing the populations of set $s$ in equation (3.1) is warranted. The 'laser piston'[150] responsible for the acceleration of hole punching particles is characterized by the strong electrostatic potential generated by the steadystate component of the laser ponderomotive force[95],

$$
\mathbf{f}_{\mathbf{l}}=\nabla\left(\frac{n_{c r}}{n_{e}} a_{0}^{2}\right) m_{e} c^{2}
$$

where $n_{e}$ is the electron density in the laser-plasma interface. We have used the three vector form since the correct four vector form of equation (3.5) remains a controversial topic of research.[202,63] $a_{0}=|e| E_{l} /\left(m_{e} c \omega_{l}\right)$ is the normalized laser vector potential, $e$ is the fundamental charge, $E_{l}$ is the laser electric field, $m_{e}$ is the electron mass, $c$ is the speed of light and $\omega_{l}$ is the laser angular frequency. $n_{c r}=m_{\mathrm{e}} \omega_{1}^{2} /\left(4 \pi e^{2}\right)$ is the critical density at which the plasma becomes opaque to the laser light. As the piston propagates into the target, we assume that background particles are swept up and fully reflected $[215,150,192,173]$. Electrons reflect from the piston head while ions are pulled in by the potential and reflect from ions bounding the piston on the downstream side. Electrons and ions are both reflected at the same velocity, $u_{i}$, due to Coulomb forces between the populations. As such, we assume they are adequately represented in momentum space as distribution functions of the same form,

$$
f_{s_{n}}(\beta)=\frac{C_{n} n_{n}}{\sqrt{2 \pi \Theta_{n}} \gamma_{i}^{3 / 2}} e^{-\frac{\left(\gamma \beta-\gamma_{i} \beta_{i}\right)^{2}}{2 \gamma_{i}^{3} \Theta_{n}}}, \quad n \in\{1,2\}
$$

representing the 'saddle point' approximation of the Jüttner distribution for $\Theta_{n} \ll 1$ 
[35]. Here, related to equation (3.1), $f_{s_{1}}$ represents the hole punching ion distribution and $f_{s_{2}}$ represents the hole punching electron distribution. $m_{n}$ is the mass, $n_{n}$ is the reflected particle density, $C_{n}$ is a normalization constant and $\Theta_{n}=T_{n} /\left(m_{n} c^{2}\right)$ where $T_{n}$ is the temperature of population $s_{n}$. $\beta=u / c$ for the particle velocity $u$ and $\beta_{i}=u_{i} / c$ for the reflected particle velocity $u_{i}$, with the Lorentz factors defined in the usual manner, $\gamma=\left(1-\beta^{2}\right)^{-1 / 2}$. This assumption is consistent with the particlein-cell simulation results, and, for many common scenarios, equation (3.6) effectively reduces to a cold Maxwellian distribution drifting at $u_{i}$.

If the phase offset $\Delta \phi$ between the laser electric field components is not precisely $\pi / 2, \mathbf{f}_{\mathbf{L}}$ also contains an oscillatory term that nonadiabatically accelerates 'strongly' relativistic electrons[95]. These 'hot' electrons are well-characterized by a MaxwellJüttner distribution[35],

$$
f_{s_{3}}(\gamma)=\frac{n_{h}}{\Theta} \frac{\gamma^{2} \sqrt{1-\gamma^{-2}}}{K_{2}(1 / \Theta)} e^{-\gamma / \Theta}
$$

where $n_{h}$ is the hot electron density, $\gamma$ is the Lorentz factor, $\Theta=T_{h} /\left(m_{e} c^{2}\right), T_{h}$ is the temperature and $K_{2}$ is the modified Bessel function of the second kind.

\subsubsection{Relation between the piston and hole punching velocities}

In a time-averaged sense, ultraintense LPI at the critical density $n_{c r}=m_{e} \omega_{L}^{2} /\left(4 \pi e^{2}\right)$ interface are characterized by the generation of a radiation pressure separation layer, comprised of electrons swept out by the laser fields, resulting in regions of charge compression and depletion. Ions are pulled along through the generation of a strong ambipolar force field, with the entire 'laser piston' structure propagating into the bulk target at the piston velocity $u_{p}$. Particle mass density is conserved as the piston sweeps up and reflects background electrons and ions. From this, the relation between 
the piston velocity $u_{p}$ and reflected ion velocity $u_{i}$ can be obtained.

By integrating over equation (3.2) in the standard fashion[79] the mass continuity equation is obtained as,

$$
\frac{\partial \Gamma_{s}^{\mu}}{\partial x^{\mu}}=0, \quad \Gamma_{s}^{\mu}=\frac{n_{s}^{\prime}}{m_{s}} P_{s}^{\mu}
$$

where $\Gamma_{s}^{\mu}=n_{s}^{\prime} \gamma_{s} c\left(1, \mathbf{V}_{\mathbf{s}} / c\right)$ is the density four vector and $n_{s}^{\prime}=n_{s} / \gamma_{s}$ is the Lorentzinvariant proper density of species $s[73]$. The ensemble average four momentum of all particles comprising species $s P_{s}^{\mu}=\gamma_{s} m_{s} c\left(1, \mathbf{V}_{\mathbf{s}} / c\right)$ is defined as,

$$
P_{s}^{\mu}=\frac{1}{n_{s}} \int p^{\mu} f_{s} \mathrm{~d} p^{k}
$$

where the Lorentz factor $\gamma_{s}=\left(1-\mathbf{V}_{\mathbf{s}} \cdot \mathbf{V}_{\mathbf{s}} / c^{2}\right)^{-1 / 2}$. The particle density $n_{s}$ is given by,

$$
n_{s}=\int f_{s} \mathrm{~d} p^{k}
$$

We evaluate equation (3.8) by changing coordinates to the rest frame of the piston. Quantities in this frame are denoted using a superscript (' $p)$. For the laboratory frame velocity $u$, the appropriate Lorentz transformation is given by[150], $u^{\left({ }^{\prime} p\right)}=(u-$ $\left.u_{p}\right) /\left(1-u \cdot u_{p} / c^{2}\right)$. Equation (3.8) can be rewritten in three vector form highlighting this choice of reference frame as,

$$
\frac{\partial}{\partial t}\left(m_{s} n_{s}^{\left({ }^{\prime} p\right)}\right)+\nabla \cdot\left(m_{s} n_{s}^{\left({ }^{\prime} p\right)} \mathbf{V}_{\mathbf{s}}{ }^{\left({ }^{\prime} p\right)}\right)=0
$$


In this frame, the interaction is steady-state and longitudinal conservation of plasma ion mass may be expressed,

$$
-M n_{i}^{\left({ }^{\prime} p\right)} u_{p}+M n_{i}^{\left({ }^{\prime} p\right)} u_{i}^{\left({ }^{\prime} p\right)}=0
$$

assuming ions are fully-reflected by the laser piston. Here $u_{p}$ is the piston velocity, $n_{i}^{\left({ }^{\prime} p\right)}$ is the ion density in the laser-plasma interface and $u_{i}^{\left({ }^{\prime} p\right)}$ is the axial velocity of the reflected ions in the piston frame. Note that equation (3.12) represents particle conservation for both the hole punching electrons and ions, as $M=M_{i}+Z m_{e}$.

From equation (3.12) it follows that $u_{i}^{\left({ }^{\prime} p\right)}=u_{p}$. Transforming back to the laboratory frame gives the hole punching ion axial velocity,

$$
u_{i}=\frac{2 u_{p}}{1+\left(u_{p} / c\right)^{2}}
$$

consistent with the Wilks et al.[215] result of $u_{i}=2 u_{p}$ in the nonrelativistic limit, i.e., ions are accelerated to twice the piston velocity, and $u_{i} \rightarrow c$ in the ultra-relativistic limit.

We now introduce the relativistic 'hot' electron beam into this framework. This is possible due to the separation of velocity scales associated with the oscillatory and steady-state components of the nonlinear force. As was suggested by Ping et al.[162], the plasma return current neutralizes the electron beam at a rate much faster than the piston velocity, i.e. $u_{h} / u_{p} \gg 1$ where $u_{h} \simeq c$ is the hot electron axial velocity. The interaction is thus steady-state on the piston timescale, such that the electron density as 'seen' by the piston is unmodified, i.e., equal to $n_{e}$. Particle number conservation 
for the hot electrons may then be written,

$$
m_{e} n_{e} u_{r}+m_{e} n_{h} u_{h}=0
$$

where $u_{r}=-\left(n_{h} / n_{e}\right) u_{h}$ is the return current velocity, i.e. a statement of the plasma neutralization of the hot electron beam current. The interplay between the laser light and the plasma near the critical interface is illustrated schematically in Fig. 3.1.

In the framework of the usual two-temperature fit to the electron $d N / d E$ energy spectrum in experimental data[214], the steady-state nonlinear force may be considered as corresponding to the low-energy component of the electron spectrum, and the oscillatory component as corresponding to the high-energy component. While the hot electrons may play a significant role energetically due to their relativistic $\gamma$-factor, in general it is expected that $n_{h} / n_{e}$ is small, as inspection of the electron energy spectrum from a typical experiment or simulation shows that the majority of the number of electrons in the system fall into the lower-energy component.

\subsubsection{Poynting theorem for ultraintense light}

Once the laser has accelerated populations $s$, their evolution is determined through conservation of phase volume given by equation (3.2). The moments of $f_{s}$ are determined by the four divergence of the electromagnetic stress-energy tensor $T^{\mu \nu}$,

$$
\frac{\partial T^{\mu \nu}}{\partial x^{\nu}}+\eta^{\mu \rho} H_{\rho}=0
$$

where the characteristic is given in equation (3.3) and $H_{\rho}=n^{\prime} d p_{\rho} / d \tau$ corresponds to the force and power densities of accelerated particles. Hence the moments of $f_{s}$,

through the ensemble average four momentum $P_{s}^{\mu}$ given by equation (3.9), enter into 
equation (3.15) through $H_{\rho}$. The stress-energy tensor is,

$$
T^{\mu \nu}=\frac{1}{4 \pi}\left(F^{\mu \alpha} F_{\alpha}^{\nu}-\frac{1}{4} \eta^{\mu \nu} F_{\alpha \sigma} F^{\alpha \sigma}\right)
$$

where $\eta^{\mu \nu}$ is the metric tensor and $F^{\mu \nu}$ is the field strength tensor as above.

In this section, we will evaluate the four conservation laws implied by equation (3.15) in Euclidean space. This approach allows us to highlight effects related to the relativistic particle fluxes central to ultraintense laser-plasma interactions, while maintaining covariant notation in the following sections as appropriate.

Consider the electromagnetic energy density $U_{e}$ in the box shown in Fig. 3.1. The Poynting theorem stipulates that,

$$
-\frac{\partial U_{e}}{\partial t}=\nabla \cdot \mathbf{S}_{\mathbf{e}}+\sum_{s} \mathbf{J}_{\mathbf{s}} \cdot \mathbf{E}
$$

where $\mathbf{S}_{\mathbf{e}}$ is the electromagnetic Poynting flux and $\mathbf{J}_{\mathbf{s}}$ is the electric current of species $s$.

As the light becomes ultraintense, the kinetic energy associated with the relativistic particle flux becomes significant. To elucidate this effect, the term in equation (3.17) corresponding to the work done against the Lorentz force can be written as,

$$
\sum_{s} \mathbf{J}_{\mathbf{s}} \cdot \mathbf{E}=\frac{\partial U_{m}}{\partial t}+\nabla \cdot \mathbf{S}_{\mathbf{m}}
$$

where $U_{m}$ is the 'mechanical' energy density in the box. $\mathbf{S}_{\mathbf{m}}$ is the mechanical Poynting vector corresponding to a sum over the particle energy flux density,

$$
\mathbf{S}_{\mathbf{m}}=\sum_{s}\left(\gamma_{s}-1\right) \mathbf{V}_{\mathbf{s}} m_{s} n_{s} c^{2}
$$


A form of Poynting's theorem useful to ultraintense laser interactions is obtained by substituting equations (3.18-3.19) into (3.17),

$$
\frac{\partial U}{\partial t}+\nabla \cdot\left(\mathbf{S}_{\mathbf{e}}+\mathbf{S}_{\mathbf{m}}\right)=0
$$

where $U=U_{e}+U_{m}$ is the total energy density in the region. Equation (3.20) states that in a steady-state interaction for the region in Fig. 3.1, the sink in the electromagnetic Poynting flux must be balanced by a source of kinetic particle flux.

It is straight forward to derive an analogous conservation law for the vector momentum flux density,

$$
\frac{\partial \mathfrak{p}}{\partial t}+\nabla \cdot\left(\mathfrak{P}_{\mathbf{e}}+\mathfrak{P}_{\mathbf{m}}\right)=0
$$

where $\mathfrak{p}$ is the total momentum density in the box in Fig. 3.1 and $\mathfrak{P}_{\mathrm{e}}$ and $\mathfrak{P}_{\mathrm{m}}$ are second rank tensors describing the flow of electromagnetic and mechanical momentum flux, i.e., corresponding to the spatial components of equation (3.15). In other words, $\nabla \cdot \mathfrak{P}_{\mathbf{e}}=\partial T^{i j} / \partial x^{j}$ and $\nabla \cdot \mathfrak{P}_{\mathbf{m}}=n^{\prime} \eta^{i j} d p_{j} / d \tau$ where $T^{\mu \nu}$ is given by equation (3.16).

We conclude this section by commenting on the dynamical nature of the laserplasma interface. The fact the interface is in motion in the laboratory frame plays an important role in satisfying equations (3.20-3.21). In general, an electromagnetic flux through a surface moving at velocity $u_{p}$ becomes,

$$
\mathbf{S}_{\mathbf{e}} \rightarrow\left(1-\frac{u_{p}}{c}\right) \mathbf{S}_{\mathbf{e}} \quad, \quad \mathfrak{P}_{\mathrm{e}} \rightarrow\left(1-\frac{u_{p}}{c}\right) \mathfrak{P}_{\mathbf{e}}
$$

In the next section, these conservation laws will be applied to determine the properties of $f_{s}$, i.e., $P_{s}^{\mu}$, for the exchange-mediating particle populations in equation (3.1). 


\subsubsection{Fully-relativistic model of ultraintense laser light absorption}

Using the tools in the preceding sections, we now develop a model of ultraintense laser light absorption by an overdense plasma. For the first time, both hot electrons and hole punching particles are described relativistically and exchange both energy and momentum with the laser.

We consider the steady-state laser-plasma interaction at the coupling stage in the laboratory frame. The ensemble average momentum and kinetic energy for each population excited by the laser are given by,

$$
\begin{aligned}
& \left\langle\mathbf{p}_{\mathbf{s}}\right\rangle=P_{s}^{k} \\
& \left\langle\mathcal{E}_{s}\right\rangle=P_{s}^{0} c-m_{s} c^{2}
\end{aligned}
$$

The bracket notation is employed to emphasize that equations (3.23-3.24) represent ensemble average quantities, i.e., no additional calculations are involved in making these definitions.

The differential energy and momentum coupled by the laser into the particles in equation (3.1) is simply,

$$
\begin{aligned}
\mathrm{d} \mathcal{E}_{s} & =N_{s} \mathrm{~d}\left\langle\mathcal{E}_{s}\right\rangle+\left\langle\mathcal{E}_{s}\right\rangle \mathrm{d} N_{s} \\
\mathrm{~d} \mathbf{p}_{\mathbf{s}} & =N_{s} \mathrm{~d}\left\langle\mathbf{p}_{\mathbf{s}}\right\rangle+\left\langle\mathbf{p}_{\mathbf{s}}\right\rangle \mathrm{d} N_{s}
\end{aligned}
$$

where $N_{s}$ is the particle number and the steady-state assumption implies that $\mathrm{d}\left\langle\mathcal{E}_{s}\right\rangle$ and $\mathrm{d}\left\langle\mathbf{p}_{\mathbf{s}}\right\rangle$ are zero.

Over time interval $\mathrm{d} t$, the number of ions accelerated by the laser piston over $\mathrm{d} t$ 
is,

$$
\mathrm{d} N_{s_{1}}=n_{i} u_{p} \mathrm{~d} A \mathrm{~d} t
$$

where $\mathrm{d} A=2 \pi r \mathrm{~d} r$ for a uniform laser spot of radius $r$. As in previous sections, $n_{i}$ is the ion density in the interface and $u_{p}$ is the piston velocity. By equation (3.12), the number of hole punching electrons excited over $\mathrm{d} t$ is $\mathrm{d} N_{s_{2}}=[Z \times$ equation (3.26)]. In this analysis we consider a 'slab' density profile, i.e. $n=n_{0}$, which is a sufficiently general representation of the overdense LP interaction. A slowly-changing heterogenous plasma profile, e.g. an exponential scalelength density profile $n(\mathbf{x})$, can be acccounted for through the straight-forward substitution $n_{0} \rightarrow n(\mathbf{x})$. This is due to the fact that each surface along a curvilinear plasma profile may be considered to be 'slab' differentially, i.e., the flux conservation is established on lengthscales smaller than the transverse intensity variation of the laser pulse.[150]. The role of large-scale underdense plasma regions is commented on briefly at the end of this section and in section 3.1.6.

The ensemble average particle energy and momentum can be related to the laserplasma kinematic exchange using equations (3.6, 3.19-3.21, 3.23-3.26). The 1-D momentum and energy flux associated with the hole punching particles can be calculated as,

$$
\begin{aligned}
& \mathcal{P}_{s_{n}}=\frac{\mathrm{dp} p_{\mathrm{n}}}{\mathrm{d} A \mathrm{~d} t}=\left\langle p_{n}\right\rangle u_{p} n_{n} \\
& \mathcal{F}_{s_{n}}=\frac{\mathrm{d} \mathcal{E}_{n}}{\mathrm{~d} A \mathrm{~d} t}=\left\langle\mathcal{E}_{n}\right\rangle u_{p} n_{n} \quad, \quad n \in\{1,2\}
\end{aligned}
$$

where $\mathcal{P}_{s_{n}}\left(\mathcal{F}_{s_{n}}\right)$ is the momentum (energy) flux. As in the previous sections, $n_{s_{1}}=$ $n_{i}, n_{s_{2}}=Z n_{i}$ 
On the other hand, the number of hot electrons $\mathrm{d} N_{s_{3}}$ excited by the oscillatory component of the laser over $\mathrm{d} t$ is not directly related to the piston dynamics. As noted in equation (3.14), particle number is conserved in the interaction with the plasma return current on a timescale fast compared to $u_{p}$. From equations (3.7) and (3.23-3.24) the hot electron momentum and energy flux densities are given by,

$$
\mathcal{P}_{s_{3}}=\gamma_{h} n_{h} m_{e} u_{h}^{2}, \quad \mathcal{F}_{s_{3}}=\left(\gamma_{h}-1\right) u_{h} n_{h} m_{e} c^{2}
$$

where $u_{h} \simeq c$ is the hot electron axial velocity. In contrast to $n_{s_{1}}$ and $n_{s_{2}}, n_{h}$ is an ansatz for the hot electron density. We assume the hot electron beam is directed longitudinally and symmetric about the beam axis. Thus, the transverse momentum terms related to equation (3.28) cancel one another on the scale $c / \omega_{p e}$. A consequence of equations (3.20-3.21) is that effects downstream of the interaction region are abstracted from the analysis, e.g., as the background plasma absorbs momentum and scatters the relativistic beam. In taking the kinematic form of equation (3.28), no assumptions are made with respect to the hot electron dynamical motion[72]. $\gamma_{h}$ corresponds to the ensemble average hot electron Lorentz factor; brackets denoting phase space averaging have hereafter been omitted for simplicity of notation.

Equations (3.27-3.28) constitute the mechanical Poynting flux $\mathbf{S}_{\mathbf{m}}$ in equation (3.20). Integrating over the volume of the box in Fig. 3.1 and invoking Gauss' theorem gives,

$$
\frac{1}{A_{b}} \iint \mathbf{S}_{\mathbf{m}} \cdot \mathrm{d} \mathbf{A}_{\mathbf{b}}=-\sum_{s} \mathcal{F}_{s}
$$

as the particle flux leaves the region on the right side. Here $\mathbf{A}_{\mathbf{b}}$ is the area of the surface bounding the box, with vector direction normal to the surface. 
Noting equation (3.22), the laser Poynting flux in equation (3.20) in integral form can be written,

$$
\frac{1}{A_{b}} \iint \mathbf{S}_{\mathbf{e}} \cdot \mathrm{d} \mathbf{A}_{\mathbf{b}}=\left(1-\frac{u_{p}}{c}\right)(1-\mathcal{R}) I_{L}
$$

where $I_{L}$ is the laser intensity reaching the overdense surface and $\mathcal{R}$ is the fraction of reflected irradiance. Using equations (3.29) and (3.30), the fully-relativistic energy flux conservation equation can be expressed,

$$
(1-\mathcal{R})\left(1-\beta_{p}\right) I_{L}=\left(\gamma_{h}-1\right) m_{e} n_{h} c^{3}+\left(\gamma_{i}-1\right) M n_{i} \beta_{p} c^{3}
$$

where $M=M_{i}+Z m_{e}$ as in the preceding sections, $\beta_{p}=u_{p} / c$ is the dimensionless piston velocity, $u_{i}$ is given by equation (3.13) and $\gamma_{i}=\left(1+\beta_{p}^{2}\right) /\left(1-\beta_{p}^{2}\right)$. The first term on the right-hand side of equation (3.31) represents coupling of the ultraintense laser light into the oscillatory mode of the nonlinear force, and the second term corresponds to coupling into the steady-state mode, the kinetic manifestation of which is the bulk reflection of electrons and ions from the piston. The fast electron current neutralization described by $\left[\left(e / m_{e}\right) \times\right.$ equation (3.14)] represents a collective response of the plasma to the beam. As noted above, the energy driving the return current is extracted from the beam itself and thus does not factor into the coupling equations.

Similarly, momentum flux conservation from equation (3.21) can be expressed,

$$
(1+\mathcal{R})\left(1-\beta_{p}\right) \frac{I_{L}}{c}=\gamma_{h} m_{e} n_{h} c^{2}+\gamma_{i} \beta_{p} M n_{i} u_{i} c
$$

where equations (3.27) and (3.28) have again been used.

Full reflection of background particles implies that the radiation pressure layer remains essentially depleted of electrons. It is thus clear that the fast electrons must 
be primarily generated at supercritical densities and may be shielded by the bulk target electrons from the full ponderomotive potential. As such, it is emphasized that $\gamma_{h}$ does not a priori follow the ponderomotive scaling[215], i.e. $\gamma_{h} \neq\left(1+a_{0}^{2}\right)^{1 / 2}$, but rather may be self-consistently determined through equations (3.31-3.32).

Employing the relation $2 I_{L} / c=m_{e} n_{c r} a_{0}^{2} c^{2}$, equations (3.32) and (3.31) may be reformulated as,

$$
\begin{aligned}
& \left(1-\beta_{p}\right)(1+\mathcal{R})=\frac{\gamma_{h}}{\beta_{0}^{2}} \frac{m_{e}}{M} \frac{n_{h}}{n_{i}}+\frac{1}{\beta_{0}^{2}} \frac{2 \beta_{p}^{2}}{1-\beta_{p}^{2}} \\
& \left(1-\beta_{p}\right)(1-\mathcal{R})=\frac{\left(\gamma_{h}-1\right)}{\beta_{0}^{2}} \frac{m_{e}}{M} \frac{n_{h}}{n_{i}}+\frac{\beta_{p}}{\beta_{0}^{2}}\left(\frac{1+\beta_{p}^{2}}{1-\beta_{p}^{2}}-1\right)
\end{aligned}
$$

where $\beta_{0}$ is the dimensionless shock velocity scale,

$$
\beta_{0}=\left(\frac{I_{L}}{n_{i} M_{i} c^{3}}\right)^{1 / 2}=\left(\frac{Z m_{e}}{2 M_{i}} \frac{n_{c r}}{n_{e}}\right)^{1 / 2} a_{0}
$$

As $\beta_{0}$ becomes closer to unity, it no longer closely approximates the actual piston velocity $\beta_{p}$. For simplicity of notation, we adopt the convention that linear light with normalized potential $a_{0}$ has identical energy density to circular light of $a_{0} / \sqrt{2}$.

$\left\{\gamma_{h}, \beta_{p}\right\}$ parameterize $f_{s}$ and thus determine the LP equilibrium condition. Problematically, however, equations (3.33-3.34) make evident the additional degree of freedom associated with the hot electrons, $n_{h}$. We treat this by defining,

$$
\bar{\rho}_{h} \equiv \frac{\rho_{h}}{\sum \rho}=\frac{Z m_{e}}{M} \frac{n_{h}}{n_{e}}
$$

where $\bar{\rho}_{h}$ represents the relative mass density in the interaction region coupled into the oscillatory mode. The $\sum \rho$ term is summed over all electrons and ions comprising the laser-plasma interface. While the electrons may play a significant role energetically 
due to the relativistic $\gamma$-factor, equation (3.36) shows that $\bar{\rho}_{h} \ll 1$, as the maximum value of $\bar{\rho}_{h} \sim Z m_{e} / M$ as $n_{h} / n_{e} \sim 1$.

Having identified the small parameter $\bar{\rho}_{h}$, we may reduce the dimensionality of the problem by taking a series expansion of the solutions to equations (3.33-3.34). To zeroth order in $\bar{\rho}_{h}$, we calculate,

$$
\beta_{p}=\beta_{0}\left(\frac{\mathcal{R}}{1+\mathcal{R} \beta_{0}^{2}}\right)^{1 / 2}
$$

Equation (3.37) is independent of $\bar{\rho}_{h}$, showing that the interplay between the piston velocity $\beta_{p}$ and light coupled into the oscillatory mode is indirect, occurring only as the fast electron absorption increases the total light absorbed by the plasma. Equation (3.37) also predicts a decrease in piston velocity as total absorption increases, with $\beta_{p} \rightarrow 0$ as $\mathcal{R} \rightarrow 0$. This limit is related to the cases described by Haines et al.[72] and Ping et al.[162].

Equation (3.37) represents the fully-relativistic hole punching velocity, taking into account both oscillatory hot electron generation and steady-state acceleration of background particles by the laser piston. This result is valid for overdense laser-plasma interactions while background ions are fully-reflected by the laser light, for all laser polarizations and fractions of reflected light $\mathcal{R}$. The parametric instabilities associated with relativistic light interacting with an underdense plasma represent additional vectors through which the light may couple to the plasma, and have been shown to lead to the formation of a supra-ponderomotive tail in the electron spectrum[36, 130]. In effect, this would increase the number of exchange-mediating populations in equation (3.1), e.g. Raman-scattering electrons[214].

It bears noting here that results from well-established absorption models follow from equation (3.37) in specific limits. The piston velocity from Wilks et al.[215], 
$\sqrt{(1+\mathcal{R}) / 2} \beta_{0}$, can be derived exactly from equation (3.32) in the limits that $n_{h} \rightarrow 0$ and $\beta_{0} \ll 1$. This expression is close to our result for $\beta_{p}$ in the nonrelativistic limit, but diverges as $\beta_{p} \gtrsim 0.1$. It is straight-forward to show that the relativisticallycorrect piston velocity for $100 \%$ conversion efficiency into ions as found by Naumova et al.[150], $u_{p} / c=\beta_{0} /\left(1+\beta_{0}\right)$, represents a contour along the surface defined by equation (3.37). Indeed, this result follows exactly from our equations (3.32) and (3.31) in the limit $n_{h} \rightarrow 0$. It should also be noted that equation (3.37) reduces in the nonrelativistic ion limit to $\sqrt{\mathcal{R}} \beta_{0}$, equivalent to the expression found in [162] for linearly-polarized light, despite quite distinct assumptions in the underlying model.

From equations (3.33-3.34) and (3.37), we find that the solution for $\gamma_{h}$ contains two terms to zeroth order in $\bar{\rho}_{h}$,

$$
\gamma_{h}=\frac{(1-\mathcal{R}) \sqrt{\beta_{0}^{2} \mathcal{R}+1}-\beta_{0} \sqrt{\mathcal{R}}(1+\mathcal{R})}{\sqrt{\beta_{0}^{2} \mathcal{R}+1} \beta_{0}^{-2}} \bar{\rho}_{h}{ }^{-1}+O(1)
$$

The $O(1)$ term is a polynomial in $\beta_{0}$ and $\mathcal{R} . \gamma_{h}$ is a moment of $f_{s 3}$ and thus represents a robust measure of the ensemble average hot electron energy. Fig. 3.2 shows the contours of the $O(1)$ term. Fig. 3.3 depicts the solutions $\left\{\gamma_{h}, \beta_{p}\right\}$ from equations (3.37) and (3.38). The ensemble average hot electron energy $\gamma_{h}$ scales as $\sim \bar{\rho}_{h}^{-1}$, while from equation (3.31) the total energy coupled into the oscillatory mode $\sim \gamma_{h} \bar{\rho}_{h}$ is independent of the parameter. This relationship is borne out by particle-in-cell simulation results, and will be discussed in more detail in sections 3.1.5 and 3.1.6.

With the piston velocity $\beta_{p}$ in equation (3.37) and the ensemble average hot electron energy $\gamma_{h}$ in equation (3.38), the distribution function moments of $f_{s}$ in equation (3.1) are fully-characterized. Together with equation (3.13) relating $u_{i}$ to the 


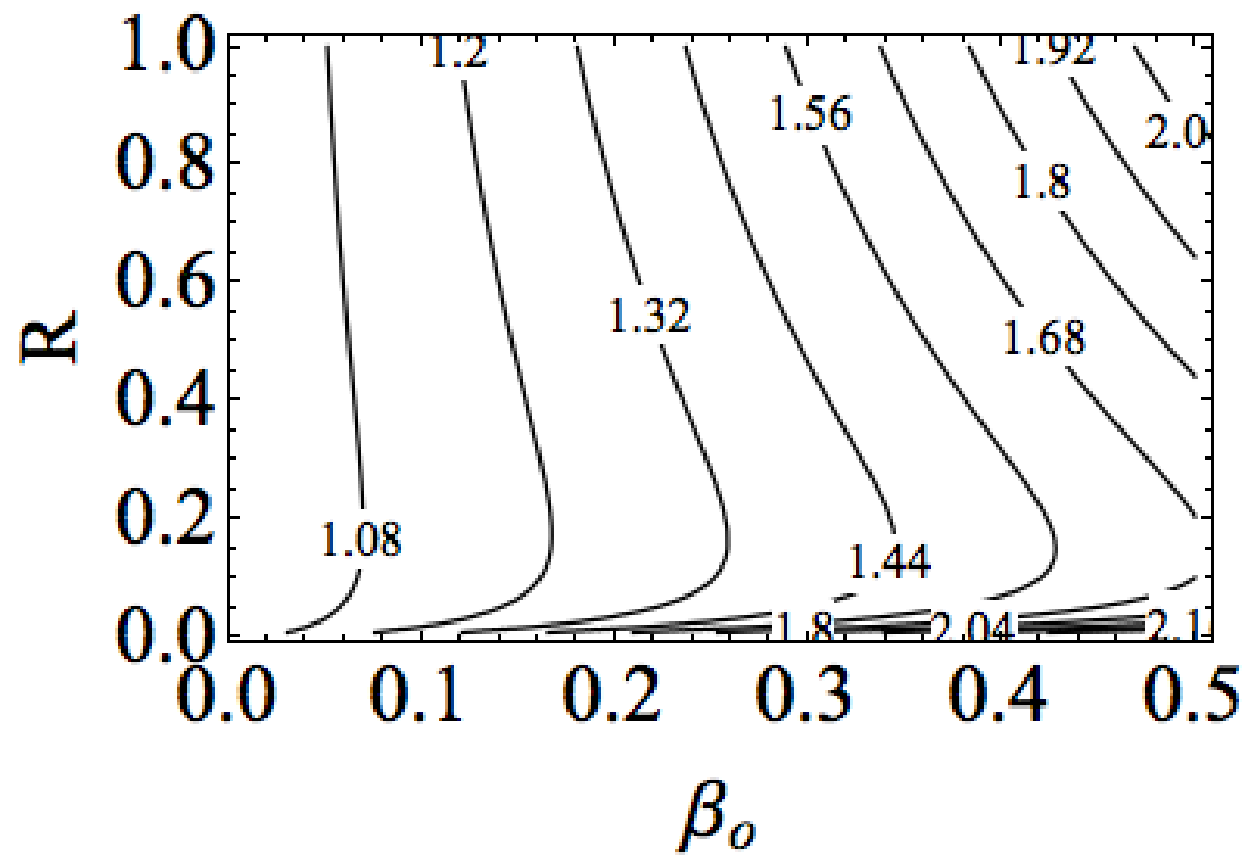

Figure 3.2 : Contours of the $O(1)$ term comprising $\gamma_{h}$ as given by equation (3.38).

piston velocity, these equations satisfy energy and momentum conservation between an ultraintense laser and overdense plasma.

\subsubsection{Conversion efficiency into hot electrons and hole punching ions}

Finally, let us consider the conversion efficiency of laser light into populations listed in equation (3.1).

From equations (3.45) and (3.37) we can calculate the conversion efficiency into hot electrons, $f_{a}^{h}$, as,

$$
f_{a}{ }^{h}=\left(\gamma_{h}-1\right) \bar{\rho}_{h} \frac{\beta_{0}^{-2}}{1-\beta_{0} \sqrt{\frac{\mathcal{R}}{\beta_{0}^{2} \mathcal{R}+1}}}
$$



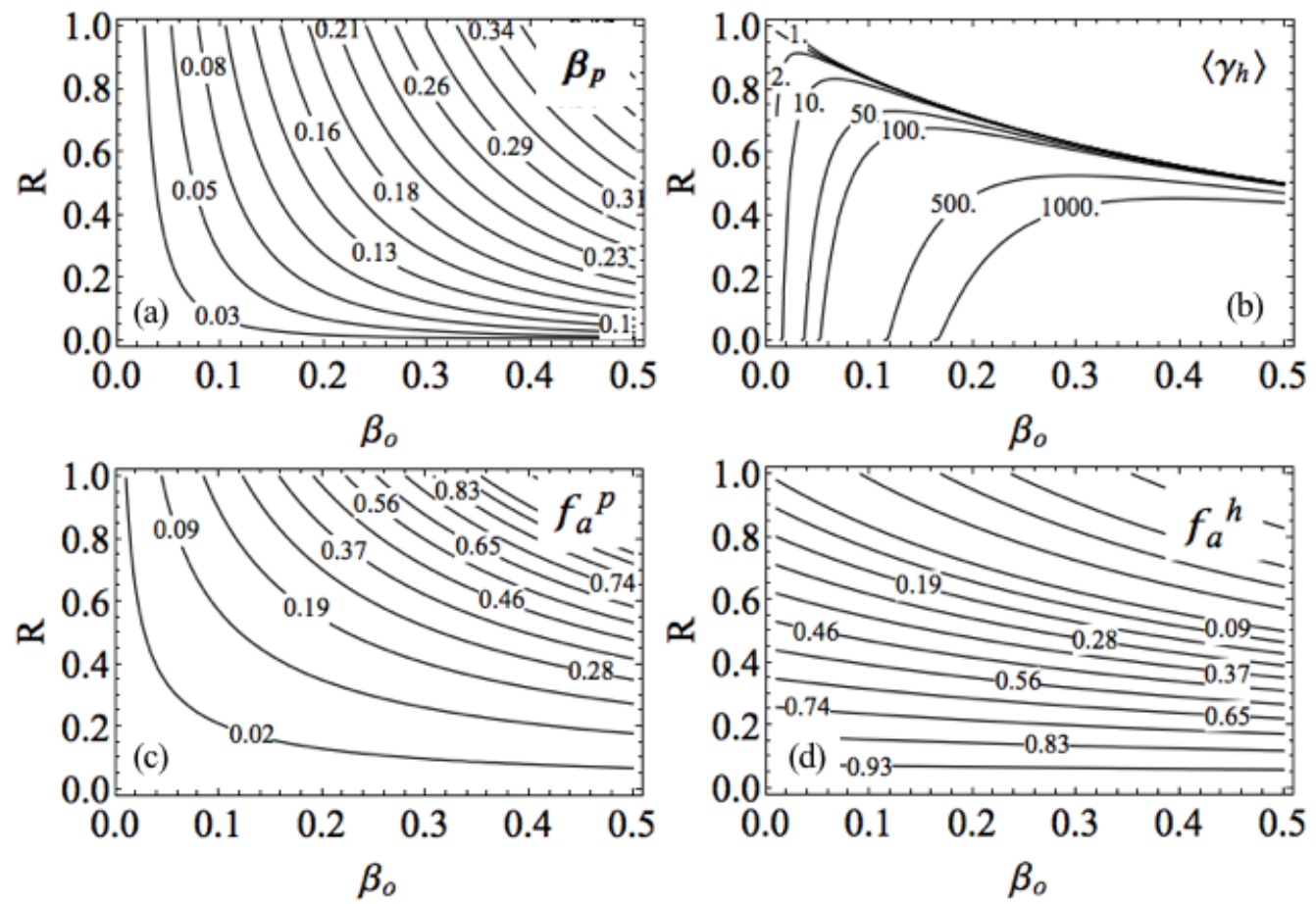

Figure 3.3 : Analytic solutions satisfying energy and momentum conservation with the laser. (a) depicts the energetic electron Lorentz factor $\gamma_{h}$ and (b) shows the piston velocity $\beta_{p}$. (c-d) show contours of the laser conversion efficiencies into ions and into energetic electrons, respectively.

Using equation (3.38), this expression can be expanded as,

$$
f_{a}{ }^{h}=\frac{(1-\mathcal{R}) \sqrt{\beta_{0}^{2} \mathcal{R}+1}-(1+\mathcal{R}) \beta_{0} \sqrt{\mathcal{R}}}{\sqrt{\beta_{0}^{2} \mathcal{R}+1}-\beta_{0} \sqrt{\mathcal{R}}}+O\left(\frac{\bar{\rho}_{h}}{\beta_{0}^{2}}\right)
$$

Equation (3.40) illustrates the key result that the total energy coupled into electrons $f_{a}{ }^{h}$ depends only on $\beta_{0}$ and $\mathcal{R}$ while $\bar{\rho}_{h} / \beta_{0}^{2} \ll 1$.

For the laser conversion efficiency into hole punching ions, $f_{a}{ }^{p}$, we have,

$$
f_{a}{ }^{p}=\frac{2 \beta_{0} \mathcal{R}^{3 / 2}}{\sqrt{\beta_{0}^{2} \mathcal{R}+1}-\beta_{0} \sqrt{\mathcal{R}}}
$$


As for $f_{a}{ }^{h}$, we observe that the conversion efficiency into hole punching ions is insensitive to the hot electron mass density $\bar{\rho}_{h}$. The conversion efficiencies from equations (3.40) and (3.41) highlight the nonlinear dependency of the coupling on the shock velocity scale $\beta_{0}$ and the total light absorption $1-\mathcal{R}$. The solutions for the conversion efficiencies are depicted in Fig. 3.3.

In concluding this section, we briefly comment on the boundary conditions associated with the total light absorption. For the case of circular polarization, corresponding to the limit $n_{h} \rightarrow 0$, the upper limit to the reflection was calculated in [150] to be $\mathcal{R}=1 /\left(1+2 \beta_{0}\right)$. Strictly-speaking, it would be incorrect to impose this limit on the general system, in which both the hot electron and hole punching absorption modes are accounted for. This will be investigated in future work, as there are additional complexities associated with deriving the limit on $\mathcal{R}$ in the general case.

In the following section we will compare the predictions of equations (3.40) and (3.41) to particle-in-cell simulations.

\subsubsection{Particle-in-cell simulations and possible astrophysical applications}

To test the predictions of the analytic model we have performed PIC simulations using the hybrid LSP code[211]. The code is configured to solve the discretized Maxwell's equations and Lorentz force equation implicitly, with no time biasing to avoid numerical damping of light waves. The timestep is determined by the Courant condition multiplied by a factor of 0.1. Electrons and ions are fully kinetic and are represented using 500 particles/cell/species. We have modified the LSP source code to implement a 'kinetic-to-kinetic' particle migration feature. This allows us to effectively distinguish, label and track electrons which interact with the laser and exceed a kinetic energy threshold from the cold background.

Simulations are one-dimensional Cartesian geometry with uniform spatial resolu- 
tion $(\Delta x)^{-1}=16$ cells $/ \mu m$ resolving the relativistic collisionless skin depth. Laser light enters the box at the left $x=0$ boundary and is incident upon an overdense $Z / A=1$ plasma slab at $x=5 \mu \mathrm{m}$. The slab density ramps to peak density $n_{0}=n_{e}=Z n_{i}$ over $0.06 \mu \mathrm{m}$ and has a $290 \mu \mathrm{m}$ spatial extent, followed by $5 \mu \mathrm{m}$ of vacuum ahead of the right boundary; the box is effectively infinite to prevent electron refluxing. The laser pulse has $1 \mu \mathrm{m}$ wavelength and rises over 3 optical cycles to a flat-top profile with 500-700fs duration. The plasma density $n_{0}$ and normalized laser amplitude $a_{0}$ are varied across simulations.

We exploit optical polarization to investigate laser coupling to the populations in equation (3.1). Starting from circular light, by 'detuning' the phase offset between the laser electric field components we can precisely examine the system as increasing energy is coupled into the oscillatory mode, as $\left|\left\langle\mathbf{f}_{\mathbf{L}}\right\rangle-\mathbf{f}_{\mathbf{L}}\right|>0$, where brackets denote a time average over the laser cyle. Our method is as follows: two lasers are injected through the $x=0$ boundary, each linearly polarized in orthogonal planes with normalized intensity $a_{0} / \sqrt{2}$. From this setup, precise control over the oscillatory electron energy is possible by tuning the relative offset between the phases of the two waves, $\Delta \phi[\mathrm{rad}]$. In this notation, $\Delta \phi=\pi / 2$ corresponds to circular light and $\Delta \phi=0$ corresponds to linear light, with the total amplitude of the light equal to $a_{0}$ for arbitrary $\Delta \phi$. We have checked that the $\Delta \phi=0$ simulations agree closely with simulations performed using a single linearly-polarized laser. This approach has an important experimental analog, as maintaining ideal polarization phases is challenging under realistic conditions.

Fig. 3.4 compares analytic predictions to simulation results for various $a_{0}, n_{0}, \Delta \phi$ configurations. (a-d) show the simulation results for the energy coupled into the oscillatory and steady-state absorption modes. Predictions from the model for $f_{a}{ }^{h}$ and $f_{a}{ }^{p}$ from equations (3.40-3.41) are overlaid on the figures, calculated using reflection 
data from the simulations at $60 \mathrm{fs}$ intervals. The absorption model agrees with the simulation data to within one percent.
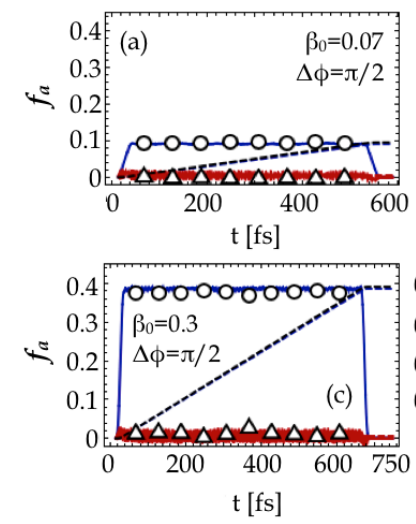
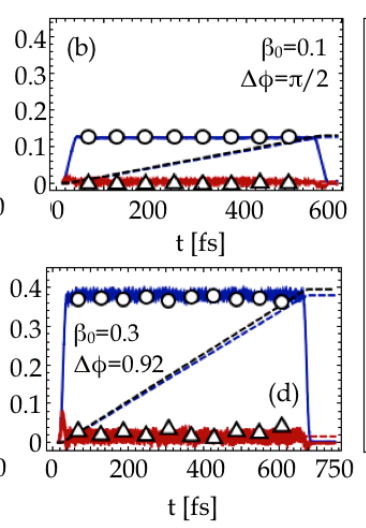

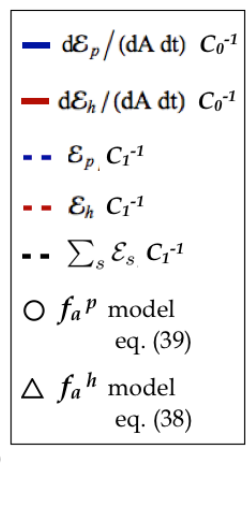

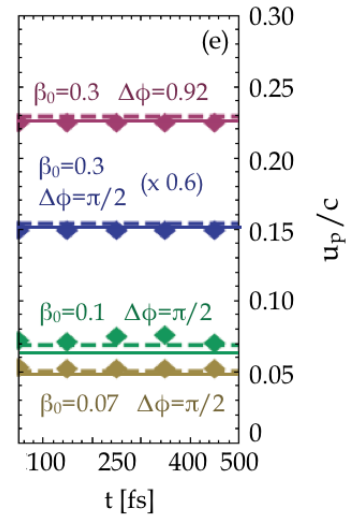

Figure 3.4 : Comparison to particle-in-cell simulations. (a-d) Light coupling into the steady-state and oscillatory ponderomotive absorption modes. (e) Piston velocity $\beta_{p}$ from the simulations and analytic model. Solid lines correspond to $d x_{p} / d t$ and diamond markers correspond to the velocity inferred from $1 \omega$ doppler shift measurements from the simulations (see text). Dashed lines represent the analytic $\beta_{p}$ from equation (3.37).

As illustrated in the legend, the cumulative particle energy is normalized to the total field energy injected into the simulation for an equivalent 'empty' run with no plasma. The simulation instantaneous particle energy flux is normalized to the attenuated injected energy flux density from the laser as noted in equation (3.32). We find that the proper normalization coefficients,

$$
\begin{aligned}
C_{0} & =\left(1-\beta_{p}\right) I_{L} \\
C_{1} & =\int I_{L} \mathrm{~d} A \mathrm{~d} t
\end{aligned}
$$

are essential so that measurements of the instantaneous coupling agree with measurements of the cumulative energy coupling, confirming equation (3.22). With this ap- 
proach, we have checked that the full time-integrated photon energy passing through the simulation boundaries agrees with the total and instantaneous energy absorbed into particles.

We note that care must be taken in measuring the instantaneous reflection using light passing through the simulation boundaries, as the energy flux associated with the reflected photons undergoes both doppler red-shifting and dispersion over time, due to the additional distance $d x=2 u_{p} d t$ traveled. Because $I_{L}$ corresponds to the laser light reaching the overdense target surface, as noted in equation (3.30), care must also be taken in scenarios with large-scale underdense plasma regions.

Fig. 3.4 (e) shows the corresponding piston velocity $\beta_{p}$ from the simulations and analytic model. The velocity from the simulation is measured using two methods: first, the location of the interface defined by $n_{i} / n_{0}=1$ is tracked at $10 \mathrm{fs}$ intervals. The solid curves represent the derivative of this 'front' or piston location over time. Second, we measure the doppler-shifting $[214,162]$ in the frequency of the $1 \omega_{L}$ light in the $E_{z}$ transverse electric field passing through the simulation boundary. The relativistic piston velocity $\beta_{p}$ is related to the frequency shift through $\Delta f / f_{0}=1-(1-$ $\left.\beta_{p}\right) /\left(1+\beta_{p}\right)$. The diamond markers represent the Fourier transformed simulation data at $100 \mathrm{fs}$ intervals. The analytic model predictions are seen to be within one percent of the simulation data across laser intensities and polarizations. Fig. 3.4 (b) depicts the $a_{0}=20 \sqrt{2}, n_{0} / n_{c r}=20, \Delta \phi=\pi / 2$ configuration, showing good agreement with results obtained in [192]. (c-d) correspond to $a_{0}=100, n_{0} / n_{c r}=30$, showing the steady-state interaction far below the relativistic critical density threshold[26]. The protons reflected from the piston in this simulation attain kinetic energy of $\sim 100$ $\mathrm{MeV}$.

Fig. 3.4 and 3.5 illustrate the effects the laser polarization offset for the $a_{0}=$ $100, n_{0} / n_{c r}=30, Z / A=1\left(\beta_{0}=0.3\right)$ plasma simulation. The electron and ion phase 
space from the simulation are depicted in 3.5 (a-b,d-e). The hot electron energy spectra across $\Delta \phi$ are depicted in Fig. 3.5 (c). In the $\Delta \phi=0.92$ simulation, the laser piston is observed to reflect background ions effectively even as $10 \%$ more energy is coupled into the oscillatory mode electrons, relative to the $\Delta \phi=\pi / 2$ simulation. Fig. 3.5 (e) compares the model predictions to the simulation results across polarizations. For the $\Delta \phi=\pi / 2,0.95,0.92$ simulations, the conversion efficiences are in agreement to $<2 \%$. As the polarization tends further towards linear, simulation results show a jump in the total energy absorbed by the plasma. This is due to the fact that the laser piston no longer fully reflects ions, allowing electrons to fall through the piston potential, i.e., the space charge-driven electrostatic potential created by the laser sweeping forward bulk electrons. Consistent with the assumptions underlying the model, accuracy falls beyond this point as additional effects such as shock acceleration of ions modify the partitioning of energy coupled into the plasma. The dynamics of these effects, recently reported on in detail[54], fall outside the scope of this section.

Laser coupling into the oscillatory absorption mode is illustrated in Fig. 3.6 for the $\beta_{0}=0.3, \Delta \phi=0.92$ simulation, corresponding to $a_{0}=100, n_{e} / n_{c r}=$ 30, $Z / A=1$. (a) depicts the density of exchange mediating electrons (red), i.e., those having interacted with the laser, and of ions (black) at two times, with the arrows indicating the dynamical position of the laser-plasma interface. The density of electrons coupled into the oscillatory mode, $n_{h}$, is calculated in the simulation as the subset of exchange-mediating electrons passing through the $x=100 \mu \mathrm{m}$ plane as shown in (b). The associated relative mass density $\bar{\rho}_{h} \ll 1$ given by equation (3.36) is labeled on the right axis. (d) depicts the ensemble average Lorentz factors for the exchange-mediating electrons and for the hot electrons. The conversion efficiency into hot electrons is examined in detail in Fig. 3.6 (c). Three quantities are depicted: first, 

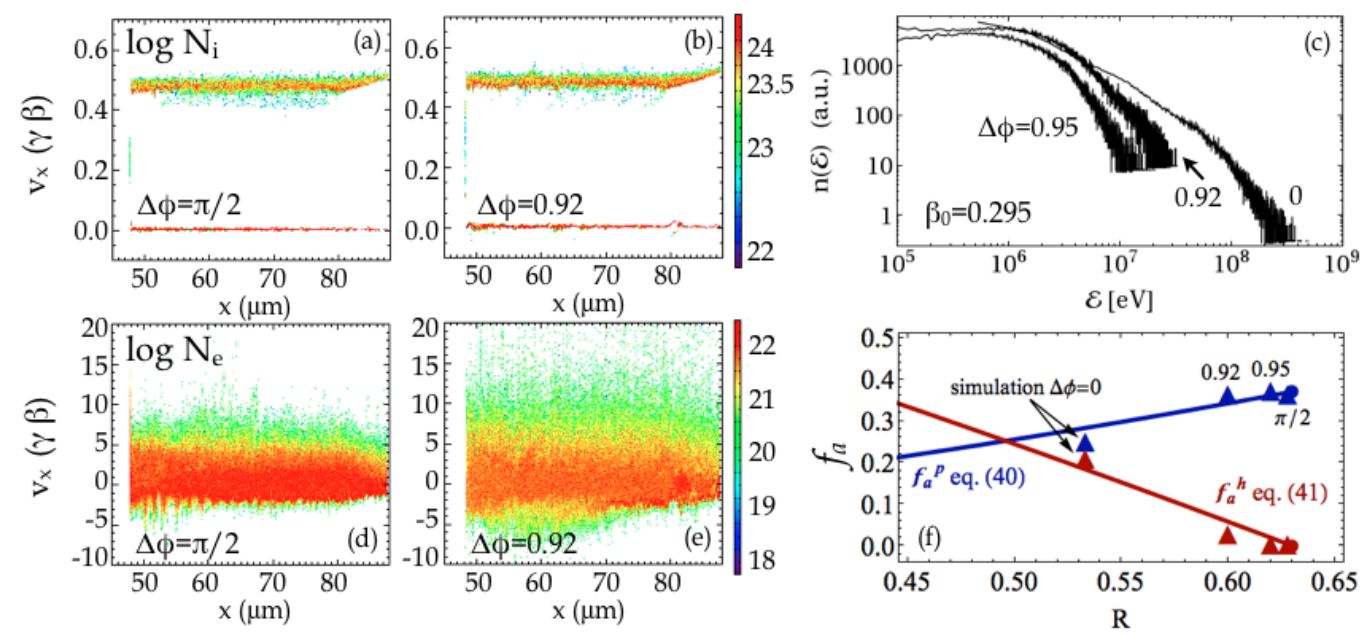

Figure 3.5 : Simulation and analytics for the $\beta_{0}=0.3$ laser-plasma interaction. (a-b) Ion and (d-e) electron phase space from simulations. (c) Hot electron spectra from simulations (relative $n(\mathcal{E})$ not to scale). (f) Comparison of laser absorption analytic predictions and simulation results.

the analytic model for $f_{a}{ }^{h}$ from equation (3.40). The dashed black curve depicts this using the parameters $\beta_{0}$ and the average reflection coefficient $\mathcal{R}=0.61$ from Fig. 3.4. Second, the hot electron energy flux density $\mathrm{d} \mathcal{E}_{h} /(\mathrm{d} A \mathrm{~d} t)$ is self-consistently calculated through a diagnostic in the simulation. This diagnostic works by calculating the instantaneous change in the total energy coupled into the particle population over time. This quantity is then normalized to the laser energy per unit time injected into a separate simulation having no plasma, accounting for the reduction in electromagnetic flux as stipulated by equation (3.42). The red curve shows this quantity normalized to equation (3.42) using the constant laser intensity $I_{L}$. Third, the expression for $f_{a}{ }^{h}$ calculated using the simulation $\gamma_{h}$ from $(\mathrm{d})$ and $\bar{\rho}_{h}$ from (b). These quantities are constrained in a well-defined fashion, according to equations (3.33-3.34). The solid black curve depicts $f_{a}{ }^{h}$ from equation (3.39) calculated using $\gamma_{h}$ and $\bar{\rho}_{h}$, confirming this relationship. In sum, Fig. 3.4 - 3.6 demonstrate excellent agreement between the 
analytic model and simulation results.
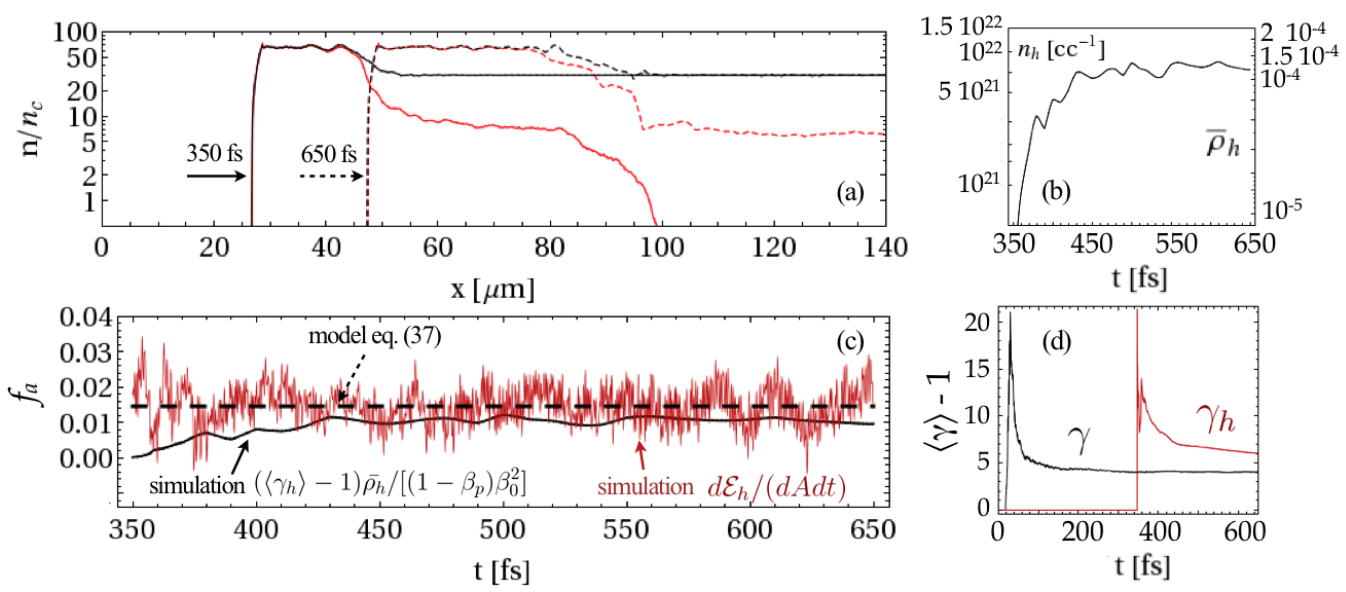

Figure 3.6 : Coupling to the oscillatory component of the laser ponderomotive force for the $\beta_{0}=0.3, \Delta \phi=0.92$ simulation. (a) Number density of exchange-mediating electrons (red) and of ions (black). Arrows indicate the position of the laser-plasma interface. (b) Hot electron density $n_{h}$ and $\bar{\rho}_{h}$. (c) Comparison of hot electron absorption from the simulation to the analytic model. (d) Ensemble average Lorentz factors (see text).

The results presented in this section for the deposition of laser energy into hole punching ion and hot electron components are germane to topical astrophysical sources. Notably, such conversion efficiencies are core unknowns in the study of astrophysical jets in gamma-ray bursts (GRBs) and blazars. Most GRBs are thought to emanate from powerful explosions of hypermassive stars located in distant galaxies in the early universe, at redshifts of around $z=1$ or larger $[165,144]$. Their enormous energy release drives a collimated, ultra-relativistic outflow (i.e., jet) with bulk Lorentz factors $\Gamma$ in excess of several hundred [4,49]. Blazars are also extragalactic jet sources, but generally nearer by and less luminous than GRBs, and with inferences of less extreme bulk motions $(\Gamma \sim 3-50)$. They emanate from the environs of persistent supermassive black holes. There is a growing enthusiasm among as- 
trophysicists for the paradigm that Poynting flux-dominated outflows in gamma-ray bursts (e.g. [126, 159]) and blazars (e.g. [221, 127] and references therein) drive their energization, precipitating radiative dissipation at large distances from their central engines. Understanding the efficiency of conversion of direct electromagnetic energy to plasma kinetic and thermal energies is therefore an extremely desirable advance. Laser-driven plasma interaction and associated kinetics can therefore provide crucial insights into these astrophysical phenomena, especially in light of the fact that the electromagnetic energy densities excited by petawatt class lasers are not widely disparate from those in blazer and GRB jets. This study and its results on laser light absorption is an important step in this direction. Formulating simple equations such as equation (3.40) to describe the ultimate kinematic apportionment of laser energy into hot electrons provides a first guide to how efficiently we think gamma-ray bursts and blazars can radiate if their outflows are mediated mostly by Poynting flux at early epochs in their expansion. Establishing a firmer relationship between the two regimes will be the subject of future work.

In review, we have developed a more comprehensive model of ultraintense laser absorption, allowing the light to couple into both the hole punching and hot electron absorption modes in an energetically significant fashion for the first time. The fullyrelativistic model has been derived for arbitrary overdense interaction densities and is insensitive to laser polarization assuming that bulk ions are swept up and fully reflected by the laser piston.

Using this framework, we have obtained solutions for the particle distribution function moments that simultaneously satisfy energy and momentum conservation, i.e. the relativistically-correct piston velocity including the hot electrons, and the hot electron Lorentz factor. For the first time, analytic expressions for the conversion efficiencies into hole punching ions and into hot electrons have been derived. 
The model establishes robust kinematic constraints on the relationships between the total absorption and the fundamental laser and plasma quantities, and recovers wellestablished results (e.g., for the piston velocity) in specific limits. Close agreement between the analytic model and particle-in-cell simulations has been demonstrated, justifying the assumptions underlying the kinematic approach. These results open the door to addressing a number of interesting ultraintense laser plasma applications in the future.

\subsection{Hot electron divergence in the kinematic analysis of rel- ativistic light on solids}

Controlling the divergence angle $\theta$ with which energetic 'hot' electrons are excited by the laser, as defined in equation (3.43) below, is central to the viability of many of the petawatt laser applications discussed in section 1 . The manner in which $\theta$ affects other aspects of the laser-plasma interaction is of further pragmatic interest. A study of the phase space aspects of such systems, related in principle to controlling the hot electron divergence[195, 176], was reported on in [108]. In this section we extend the quasi-1-D absorption model developed in section 3.1 to 1D2V (one spatial coordinate, two velocity coordinates), thereby allowing hot electrons to take on an axisymmetric divergence. This is significant as the manner in which the hot electron divergence angle $\theta$ affects other aspects of the laser-plasma interaction can determine the viability of applications in $[136,204]$, and beyond. We present an analytic examination of how key interaction parameters, specifically, the hp velocity $u_{p}$, the hot electron Lorentz factor $\gamma_{h}$, the coupling efficiency into hp ions $f_{p}$ and the coupling efficiency into hot electrons $f_{h}$, change as $\theta$ increases. Across all laser and plasma parameters, we show that absorption into hp ions $f_{p}$ increases as $\theta$ increases. In the nonrelativistic limit, 
we derive a general analytic formula for $u_{p}$ in terms of $\theta$, the fractional reflection $\mathcal{R}$, and laser and plasma parameters.

We examine the steady-state laser-plasma interaction, in which an ultraintense laser having infinite transverse extent, intensity $I_{L}$ and wavevector $\mathbf{k}_{\mathbf{L}}$ is normally incident onto an overdense target with arbitrary, slowly-changing heterogenous plasma density profile[150]. The 'laser piston' sweeps up and fully reflects hp particles along $\hat{k}_{L}[150]$. All particles in the laser-plasma (LP) interface are assumed to interact with the laser and accelerate by coupling into the steady-state or oscillatory mode of the nonlinear force.

In the quasi-1-D, fully-relativistic absorption model developed in section 3.1, two assumptions were made with respect to the hot electron beam distribution function as it is excited by the laser. First, it was assumed that the beam was symmetric about the longitudinal axis. No factor in our simple system exists to break this symmetry, and we maintain this assumption here. Second, it was assumed that the axial component of the hot electron velocity satisfied $\beta_{\|} \simeq 1$, where $\overrightarrow{\beta_{h}}=\left(\beta_{h, \|}, \beta_{h, \perp}\right)$ is the ensemble average hot electron velocity, i.e., as given by equation (3.9) above, employing standard unit bases in the axial (parallel) and transverse (perpendicular) directions relative to electron motion.

In this section, we relax this latter assumption and consider how key dynamical parameters of the interaction change when $\beta_{\|}<1$. In this effort the assumption of $\beta_{\|} \simeq 1$ is discarded and the general form of $\beta_{h, \|}=\cos \theta$ is adopted, where

$$
\theta \equiv \tan ^{-1} \beta_{h, \perp} / \beta_{h, \|}
$$

is the half-angle with which the hot electrons are excited by the laser. Equivalently $\theta=\tan ^{-1} p_{\perp} / p_{\|}$for electron momentum $\vec{p}=\gamma_{h} \overrightarrow{\beta_{h}} m_{e} c$ where $m_{e}$ is the electron 
mass and $c$ is the speed of light. We emphasize that the observed divergence angle in experiment will generally differ from $\theta$, due to downstream effects beyond the LP interface, e.g., scattering in the plasma bulk, which are abstracted from this analysis. Streaming instabilities which couple the hot electrons to the cold plasma return current may also occur in certain scenarios, which act to increase the beam emittance. We assume these flow instabilities cannot 'see' the intense electromagnetic fields of the laser, taking place just beyond the relativistic collisionless skin depth. This assumption is readily satisfied since all particles in the interface interact with the laser and are accelerated from the skin layer. Hence the particles in the interface are continually 'refreshed,' i.e., particles comprising the interface at time $t_{0}$ are different than those at $t_{0}+d t$, meaning that instabilities cannot grow. Furthermore, transverse instabilities associated with the hot electrons exhibit inverse wavenumbers of $c / \omega_{p e}$ or larger[10]. Thus, the growth of these instabilities is quite generally supressed.

Following the methodology developed in section 3.1 the conservation laws relevant to this system are calculated as,

$$
\begin{aligned}
& \left(1-\beta_{p}\right)(1+\mathcal{R})=\frac{\gamma_{h}}{\beta_{0}^{2}} \frac{m_{e}}{M} \frac{n_{h}}{n_{i}} \beta_{h, \|}^{2}+\frac{1}{\beta_{0}^{2}} \frac{2 \beta_{p}^{2}}{1-\beta_{p}^{2}} \\
& \left(1-\beta_{p}\right)(1-\mathcal{R})=\frac{\left(\gamma_{h}-1\right)}{\beta_{0}^{2}} \frac{m_{e}}{M} \frac{n_{h}}{n_{i}} \beta_{h, \|}+\frac{\beta_{p}}{\beta_{0}^{2}}\left(\frac{1+\beta_{p}^{2}}{1-\beta_{p}^{2}}-1\right)
\end{aligned}
$$

for momentum flux and energy flux, respectively. These equations are mathematically equivalent to equations (3.33-3.34) above under the transformation of the hot electron axial velocity $u_{h} \rightarrow c \cos \theta$ using equation 3.43. Here $\beta_{p}=u_{p} / c$ is the piston (hole punching) velocity, $\mathcal{R}$ is the fractional reflected light, $f=1-\mathcal{R}$ is the total fractional absorbed light, $\gamma_{h}=\left(1-\overrightarrow{\beta_{h}} \cdot \vec{\beta}_{h}\right)^{-1 / 2}$ is the hot electron Lorentz factor, $M$ is the ion mass, $n_{h}$ is the number density of hot electrons in the laser-plasma (LP) interface and 
$n_{i}$ is the total ion density in the LP interface. $\beta_{0}$ is the dimensionless Wilks velocity as given above by,

$$
\beta_{0}=\left(\frac{I_{L}}{n_{i} M_{i} c^{3}}\right)^{1 / 2}=\left(\frac{Z m_{e}}{2 M_{i}} \frac{n_{c}}{n_{e}}\right)^{1 / 2} a_{0}
$$

where $I_{L}$ is the laser intensity, $Z$ is the average charge state in the LP interface and $a_{0}=e E_{L} /\left(m_{e} c \omega_{L}\right)$ is the normalized vector potential.

We now solve equations (3.44-3.45) numerically in order to study how finite divergences of the hot electron beam affect the solutions for the piston velocity $\beta_{p}$, hot electron gamma factor $\gamma_{h}$, conversion efficiency into hots $f_{h}$ and conversion efficiency into hole punching ions $f_{p}$. Fig. 3.7 and 3.8 depict solutions obtained from equations (3.44-3.45) for a range of parameters, as a function of the total fractional reflection $\mathcal{R}$ and the dimensionless shock velocity scale $\beta_{0}$. The variables $\left(\beta_{0}, \mathcal{R}\right)$ in principle form the basis for all ultraintense laser-overdense plasma interactions[106]. Therefore, we expect a substantial variance in the form of the curves in the Fig. 3.7 and 3.8 . However, there is one clear trend which can be elucidated. 

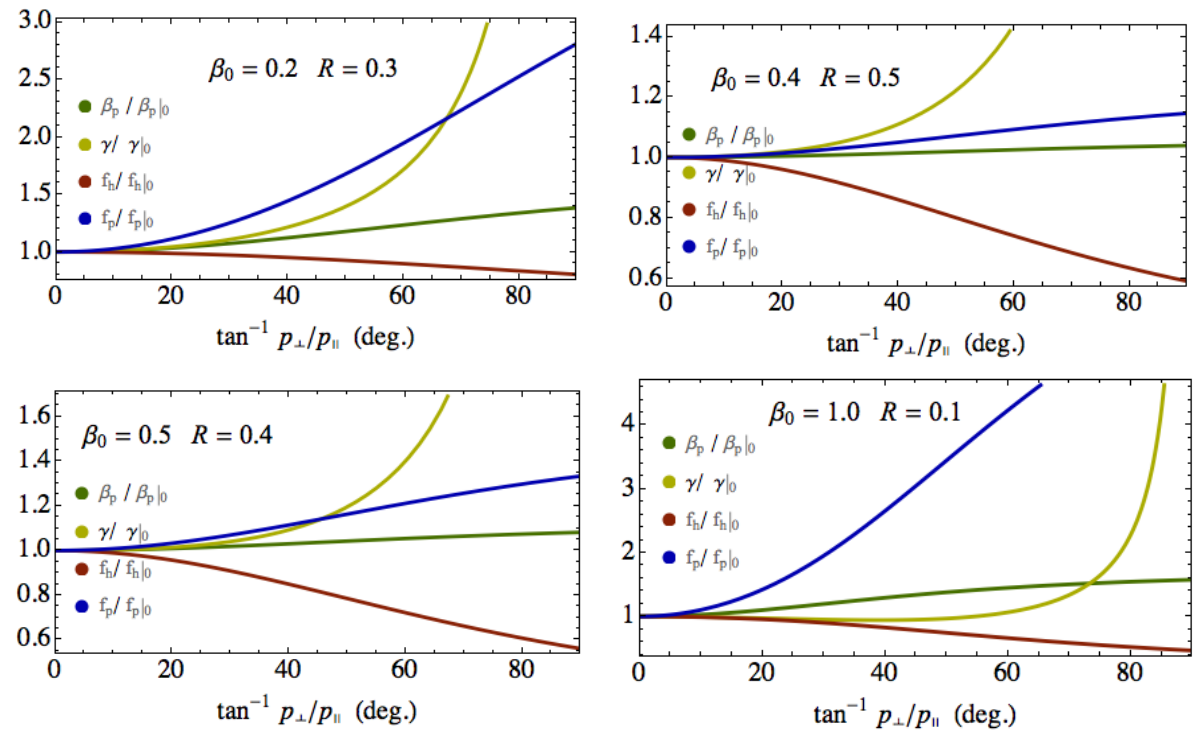

Figure 3.7 : Relative changes to solutions as a function of the hot electron beam divergence angle $\theta$. Quantities are normalized to their $\theta=0$ values.
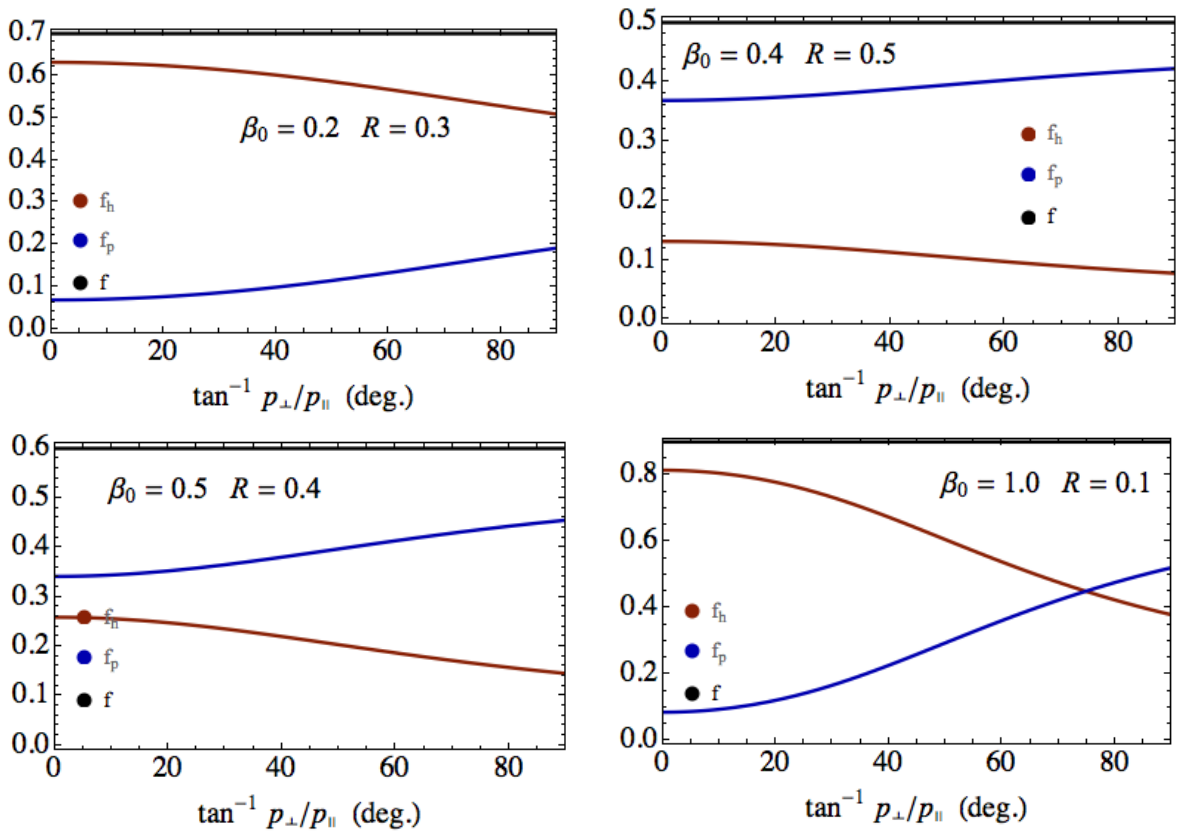

Figure 3.8 : Absolute changes in energy partitioning as a function of $\theta$. 
We observe that the absorption into ions increases as $\theta$ increases. This is because as the projection of the hot electron momentum flux in the axial direction becomes smaller, ions must be pushed faster to conserve momentum with the laser. Consistent with this, calculations indicate that there is a 'minimum absorption' into ions. This picture is appropriate for any realistic hot electron beam divergence, $\theta<60^{\circ}$, as shown in Fig. 3.7 for a variety of laser and plasma parameters.

On the other hand, considering the asymptotics, we see that a strongly-diverging hot electron system must approach the ion-only system described in [215, 150]. From equations (3.44-3.45) it is clear that as $\theta \rightarrow \pi / 2$ (corresponding to hot electron emission into full $\pi$ radians), the terms corresponding to the hot electron energy and momentum flux vanish, recovering the relativistic ion model in [150]. However, solutions from equations (3.44-3.45) for $\gamma_{h}$ indicate that the Lorentz factor begins to diverge, typically around $\theta>65-75^{\circ}$, as shown in Fig. 3.7. This nonphysical behavior in $\gamma_{h}$ occurs in an attempt to cancel the reduction in $\beta_{h, \|}$. In reality, the hot electrons cannot continue to accelerate, and $f_{h} \rightarrow 0$ per the above considerations. In effect, the kinematic analysis is indicating that a longitudinal force cannot excite such a strongly diverging beam. This will be considered with further detail in future work.

Finally, we note one additional interesting consequence of the above analysis. In the nonrelativistic limit, independent of the diverging $\gamma_{h}$, an analytic form for the piston velocity $\beta_{p}$ can be derived,

$$
\beta_{p}=\frac{\sqrt{1+\mathcal{R}-(1-\mathcal{R}) \cos \theta}}{\sqrt{2}} \beta_{0}+O\left(\beta_{0}^{2}\right)
$$

Equation (3.47) generally sheds light on the hole punching dynamics[120, 162, 83] in ultraintense laser-plasma interactions, recovering the result from Wilks et al.[215] 
when $\theta=\pi / 2$, as well as the result in [106] when $\theta=0$. The relativistic form of $\beta_{p}$, as well as applications of equation (3.47), will be studied in future work.

In review in this section we have presented an analytic examination of the effects of a strongly diverging hot electron beam on key aspects of the laser-plasma interaction. We have shown that absorption into hole punching ions increases with $\theta$, the hot electron divergence half-angle, across laser and plasma parameters. In the nonrelativistic limit, a general analytic formula for the hole punching velocity in terms of $\theta$, the fractional reflection, and laser and plasma parameters has been derived. Future work will focus on examining higher-dimensional effects.

\subsection{Simple causal model of electron angular divergence in ultraintense laser-plasma interactions}

As covered in the previous section, the divergence of the hot electron beam excited in relativistic laser-plasma interactions is of fundamental interest to fast ignition and many other petawatt laser applications[160]. Section 3.2 described a theoretical approach to treating the electron divergence in the framework of absorption, in a manner that is insensitive to the underlying physical mechanisms causing the divergence. While the value of this analysis is not in doubt, it is of additional use to understand the dominant physical mechanisms in order to mitigate the beam divergence. In this section we develop a simple causal model to describe the electron divergence $d N / d \theta$ for a given laser driver and target.

The ponderomotive 'rippling' of the critical interface[215], as covered in section 1.2.1, plays a central role in determining the distribution function of electrons injected into the target. This is due to the enhanced laser absorption that comes about as a result of the changing angle between the laser electric field and target normal. 
Electrons injected into the bulk target are also often thought of as comprising a beam of charged particles exhibiting a classical predisposition towards plasma instabilities arising in the interaction with the cold return current[53]. These instabilities act to reduce the free energy of the injected particles, modifying the beam properties and increasing its divergence.

In this section we develop a simple model that represents the hot electron injection into the target as the sum of numerous discrete 'beams' generated at each point in space at the spatio-temporally evolving critical interface. While for simplicity we consider linearly polarized laser light interacting with a dense plasma at normal incidence, effects relating to the laser polarization and incidence angle are captured by the $\vec{E}_{L}$ vector. There are two components we need to do this: (1) the absorption efficiency of the various laser mechanism as a function of system parameters, e.g. $I_{L}, \hat{n}\left(\overrightarrow{x_{f}}, t\right) \cdot \overrightarrow{E_{L}}$ for surface normal unit vector $\hat{n}\left(\overrightarrow{x_{f}}, t\right)$; and $(2)$ the spatial structure of the critical interface, $\overrightarrow{x_{f}}(\vec{x}, t)$. At each point along the critical surface, both the fraction of laser energy coupled into hot electrons, i.e. the normalized energy flux density $P_{e} / P_{L}$, and the directionality of the injected electrons may then be determined, for energy flux density of electrons $P_{e}$ and of the laser $P_{L}$. This is depicted schematically in Fig. 3.9.

Let us first treat requirement (1). Here we will consider contributions to electron heating from the $\vec{J} \times \vec{B}[95]$ and Brunel (vacuum heating) [24] mechanisms, believed to play dominant roles in the regimes of interest. For the vacuum heating mechanism, the component of the laser electric field normal to the target surface rips electrons out of the target into vacuum, and accelerates and reinjects them into the target along the density gradient. The relevant expressions for the laser absorption through this 
$t_{0}$

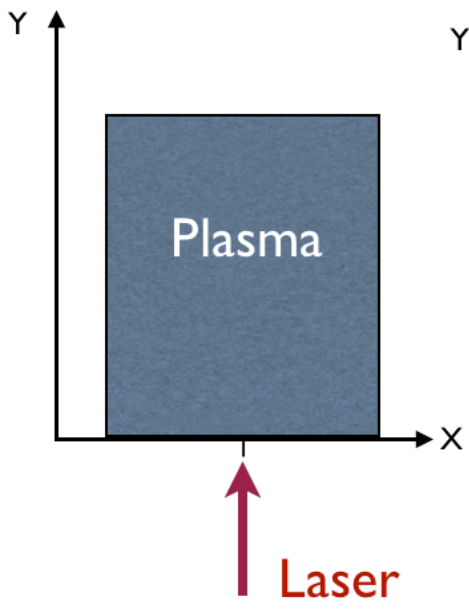

$t_{1}$

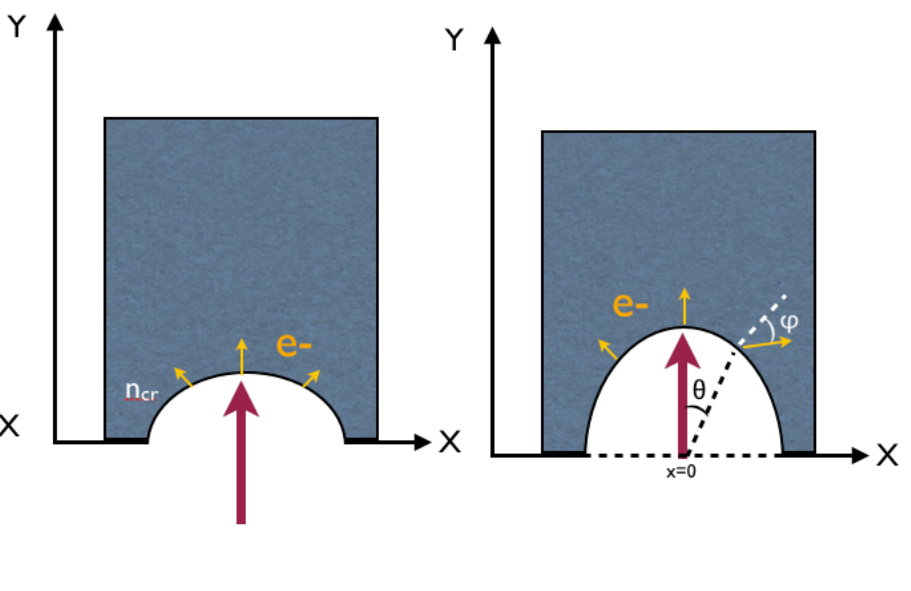

Figure 3.9 : Schematic depicting the evolution of the plasma critical surface over time for an ultraintense laser pulse having Gaussian transverse spatial profile. Electron generation is also depicted. In $(\mathrm{C})$, we see the unit vector normal to the critical surface as a function of radial position and time, $\hat{x_{f}}(\theta, t)$. The directionality $\hat{\phi}$ and magnitude $P_{e} / P_{L}$ of the hot electrons relative to $\hat{x_{f}}$ at each spatial point is a function of laser parameters. 
process is given in the literature as[24],

$$
\begin{array}{r}
f_{a b s, v h}=\frac{\eta}{2 \pi} \frac{v_{o s c}^{3}}{v_{L}^{2} c \cos \theta} \\
\overrightarrow{F_{v h}} / F_{v h}=\hat{E}_{L}
\end{array}
$$

where $v_{L}=e E_{L} /\left(m_{e} \omega_{L}\right)$ and $v_{o s c}=e E_{0} /\left(m_{e} \omega_{L}\right)$. Here $E_{0}$ is component normal to surface, i.e. $E_{0}=2 E_{L} \sin \theta$ for a perfect plasma conductor. The density dependence is included through $\eta \simeq\left(1-\omega_{L}^{2} / \omega_{p e}^{2}\right)^{-1}$.

For the $\vec{J} \times \vec{B}$ process, it is shown in the literature for the nonrelativistic case[214] that,

$$
\overrightarrow{F_{p}}=\frac{\partial}{\partial x}\left[\frac{m_{e} a_{0}^{2} c^{2} \omega_{L}^{2}}{\omega_{p e}^{2}} e^{-2 \omega_{p e} x / c}\left(1+\cos 2 \omega_{L} t\right)\right] \hat{k_{L}}
$$

Here $a_{0}=e E_{L} /\left(m_{e} \omega_{L} c\right)$ is the dimensionless laser parameter. The constant component yields the ponderomotive 'laser pressure' force and the oscillatory component corresponds to nonadiabatic $\vec{J} \times \vec{B}$ electron heating.

With respect to requirement (2), the evolution of the critical surface is given in [110] in one dimension. For a semi-infinite pulse of intensity $I_{0}$ incident on an exponential density gradient $n_{0}(x)=n_{0} \exp \left[\ell^{-1}\left(x-x_{s}\right)\right]$ with scalelength $\ell=n(d n / d x)^{-1}$ and critical surface at $x_{s}$, we note $u_{f}=(d / d t) x_{f}$ and employ separation of variables to solve for the front motion,

$$
x_{f}(t)=2 \ell \ln \left[1+e^{x_{s} /(2 \ell)} \frac{u_{w 0}}{2 \ell} t\right]
$$

where $u_{w 0} \equiv\left[(1+\eta) I_{0} /\left(2 M_{i} n_{0} c\right)\right]^{1 / 2}=\left(a_{0} c / 2\right)\left[(1+\eta) Z m_{e} n_{c r} /\left(2 M_{i} n_{p e 0}\right)\right]^{1 / 2}$ is the characteristic (constant) Wilks velocity for the system. 
For a realistic pulse profile, here a temporal Gaussian of the form $I(t)=I_{0}(t) \times$ $\exp \left[-2\left(t-\alpha t_{1 / 2}\right)^{2} / t_{1 / 2}^{2}\right]$ where $\alpha$ determines $I\left(t_{0}\right)$, interacting with an exponential scale length plasma. The coefficient $\partial_{t} I_{0}(t) \neq 0$ may be employed to describe nonlinear effects, e.g. laser self-focusing[46] for $P_{L} / P_{c}=P_{L}\left(17 G W n_{c r} / n_{p e 0}\right)^{-1}>1$. Assuming constant $I_{0}$, a closed-form solution may be obtained,

$$
x_{f}(t)=2 \ell \ln \left[1+e^{x_{s} /(2 \ell)} \frac{\sqrt{\pi} u_{w 0} t_{1 / 2}}{4 \ell} \Omega(t)\right]
$$

where $\Omega(t) \equiv \operatorname{erf}(\alpha)+\operatorname{erf}\left[\left(t-\alpha t_{1 / 2}\right) / t_{1 / 2}\right]$ and the error function is employed. As noted in [110] it is straight-forward to generalize the $x_{f}(t)$ expressions to three dimensions.

Taken together, the above expressions determine both the directionality and magnitude of the electron generation at each point along the time-evolving critical surface. For symmetry in azimuthal angle, the Cartesian spatial dependence of the electron generation is given by,

$$
\vec{P}_{e}(\theta)=P_{L}(\theta) \sum_{i} f_{a b s, i}\left(\vec{P}_{L}(\theta), \theta\right) \hat{\epsilon}_{i}
$$

where the radial coordinate $x$ maps to $\theta, i \in\{v h, j x b, .$.$\} and \hat{\epsilon}_{i}$ is the directionality associated with each mechanism. This expression may be related directly to the desired angular divergence $d N / d \theta \sim P_{e}(\theta)$.

In summary, we have outlined the development of a simple model treating electron $d N / d \theta$ in ultraintense laser plasma interactions where the dominant contributions to hot electron divergence are related to the rippling of the critical interface. At each spatial point, the relative number of electrons accelerated $P_{e} / P_{L}$ is a function of laser intensity, plasma density and the angle between the laser electric field and $\hat{x_{f}}$, through 
the expressions for laser absorption efficiency above. The directionality of the hot electrons accelerated at each point is intrinsic to each laser absorption mechanism, i.e.

$\hat{k_{L}}$ for $\vec{J} \times \vec{B}$ or $\hat{E}_{L}$ for Brunel. Contributions from absorption processes are summed at each point along the critical surface, such that any given point acts as a source for both forward-going and divergent electrons. Further development of this model could lead to an enhanced understanding of the mechanisms driving the electron beam divergence in petawatt laser plasma interactions, and ultimately provide insights into approaches for its mitigation.

\subsection{Relativistic plane wave model of electron beam genera- tion}

Further motivated by the centrality of the electron energy to relativistic laser applications, it is instructive to consider a more detailed model of electron acceleration. For clarity we conceptualize the laser as a relativistic monochromatic plane wave propagating in $\hat{z}$, impinging on a high density plasma located at $z=0$, a model which has underpinned many insights into laser-plasma interactions $[92,72,174,3]$. The laser has the four vector potential $A^{\mu}=(0, \vec{A})$ where $\vec{A}$ is given by,

$$
\vec{A}=a_{0} \sin \left[\frac{2 \pi}{\lambda_{l}}(c t-z)+\psi\right] \hat{x}
$$

where $\psi$ is the initial phase of the wave. The electromagnetic fields follow from $\vec{A}$ as,

$$
\vec{E}_{l}=-\frac{m_{e} c}{|e|} \frac{\partial \vec{A}}{\partial t}, \quad \vec{B}_{l}=\frac{m_{e} c^{2}}{|e|} \vec{\nabla} \times \vec{A}
$$


Performing the elementary differentiation we find that,

$$
\begin{aligned}
& \vec{E}_{l}=-\frac{2 \pi}{\lambda_{l}|e|} a_{0} m_{e} c^{2} \cos \left[\frac{2 \pi(c t-z)}{\lambda_{l}}+\psi\right] \hat{x} \\
& \vec{B}_{l}=-\frac{2 \pi}{\lambda_{l}|e|} a_{0} m_{e} c^{2} \cos \left[\frac{2 \pi(c t-z)}{\lambda_{l}}+\psi\right] \hat{y}
\end{aligned}
$$

Equations (3.56-3.57) are controlled by the dimensionless wave phase,

$$
\varphi=\frac{c t-z}{\lambda_{l}}
$$

For our analysis an energy conservation equation is required in addition to the Lorentz force, which is derived by taking the scalar product of $\vec{p}$ with equation (1.4) as,

$$
m_{e} c^{2} \frac{d \gamma}{d t}=-|e| \frac{\vec{p}}{\gamma m_{e}} \cdot \vec{E}_{l}
$$

Having fully specified the electromagnetic fields the electron dynamics can now be analyzed. In this effort we adopt the convenient units that $c=m_{e}=|e|=1$. Using equations (1.4) and (3.59) we calculate the evolution of the electron phase space to be,

$$
\begin{aligned}
\frac{d p_{x}}{d t} & =\frac{2 \pi}{\lambda}\left(1-\frac{p_{z}}{\gamma}\right) a_{0} \cos (2 \pi \varphi+\psi) \\
\frac{d p_{y}}{d t} & =0 \\
\frac{d p_{z}}{d t} & =\frac{2 \pi}{\lambda} a_{0} \frac{p_{x}}{\gamma} \cos (2 \pi \varphi+\psi) \\
\frac{d \gamma}{d t} & =\frac{2 \pi}{\lambda} a_{0} \frac{p_{x}}{\gamma} \cos (2 \pi \varphi+\psi)
\end{aligned}
$$

highlighting that particle momentum along the magnetic field vector $\hat{B}_{l}$ is conserved. From the relation that $\gamma=\left(1+p_{x}^{2}+p_{y}^{2}+p_{z}^{2}\right)^{1 / 2}$ and equations (3.62-3.63) it is clear 
that $d p_{z} / d t=d \gamma / d t$. We assume that the electron is initially at rest which yields the well known relation,

$$
p_{z}=\gamma-1
$$

To obtain $p_{x}$ we transform coordinates $t \rightarrow \varphi$ using equation (3.58), yielding,

$$
\frac{d p_{x}}{d t}=\frac{1}{\lambda_{l} \gamma} \frac{d p_{x}}{d \varphi}
$$

Equation (3.65) yields $p_{x}$, and thus the dimensionless four-momentum of electrons in this system $p^{\mu}=(\gamma, \vec{p})$ is given by,

$$
p^{\mu}=\left(1+\frac{a_{0}^{2}}{2} \sin ^{2}(2 \pi \varphi+\psi), a_{0} \sin (2 \pi \varphi+\psi), 0, \frac{a_{0}^{2}}{2} \sin ^{2}(2 \pi \varphi+\psi)\right)
$$

and $p^{\mu} p_{\mu}=-1$ is a Lorentz scalar as it should be.

We are now interested in determining the ensemble averaged properties, specifically the energy, of electrons described by equation (3.66). Following ref. [92] we consider the electron distribution function,

$$
\frac{d N_{e}}{d t}=\frac{d N_{e}}{d \varphi} \frac{d \varphi}{d t}
$$

Assuming that the laser temporal profile ramps from zero in an adiabatic fashion, electrons will be uniformly distributed with respect to the wave phase. Since $d \varphi / d t \propto$ $1 / \gamma$,

$$
\frac{d N_{e}}{d \varphi}=\lambda_{l} \frac{d N_{e}}{d \tau}=\text { const. }
$$


where the electron proper time $d \tau=1 / \gamma d t$. This is due to the fact that the change in phase $d \varphi$ over $d \tau$ is independent of the electron energy $\gamma$. Equation (3.68) will hold for electrons accelerated by any mechanism satisfying equation (3.64). For these situations the ensemble-average electron energy $\langle\gamma\rangle$ can be written,

$$
\langle\gamma\rangle=\frac{\int_{0}^{t(\varphi=2 \pi)} \gamma \frac{d N_{e}}{d \varphi} \frac{d \varphi}{d t} d t}{\int_{0}^{t(\varphi=2 \pi)} \frac{d N_{e}}{d \varphi} \frac{d \varphi}{d t} d t}
$$

which using equation (3.68) reduces to the expression,

$$
\langle\gamma\rangle=\frac{2 \pi}{\int_{0}^{t(\varphi=2 \pi)} \frac{1}{\gamma} d t}
$$

According to equation (3.70) the average electron Lorentz factor is $\gamma$ averaged over the laser phase, or equivalently, the electron proper time.

Equation (3.70) can be readily evaluated with $\gamma=p^{0}$ from equation (3.66). However, we first recover the results of Kluge et al.[92] by instead using the transverse Lorentz factor $\gamma_{t}=\left(1+p_{x}^{2}+p_{y}^{2}\right)^{1 / 2}=\sqrt{1+a_{0}^{2} \sin ^{2}(2 \pi \varphi+\psi)}$. This calculation yields equation (1.17) from section 1.2.1.

We now extend these results by calculating $\langle\gamma\rangle$ using the full Lorentz factor from equation (3.66). Computing the integral in equation (3.70) using $\gamma=p^{0}$ yields,

$$
\langle\gamma\rangle_{p \|+p \perp}=\frac{2 \sqrt{2} \pi^{2} \sqrt{a_{0}^{2}+2}}{13 \pi+\tan ^{-1}\left(\frac{\sqrt{a_{0}^{2}+2} \tan \left(4 \pi^{2}\right)}{\sqrt{2}}\right)}
$$

Including the calculations reviewed in section 1.2.1 the effective hot electron tem- 


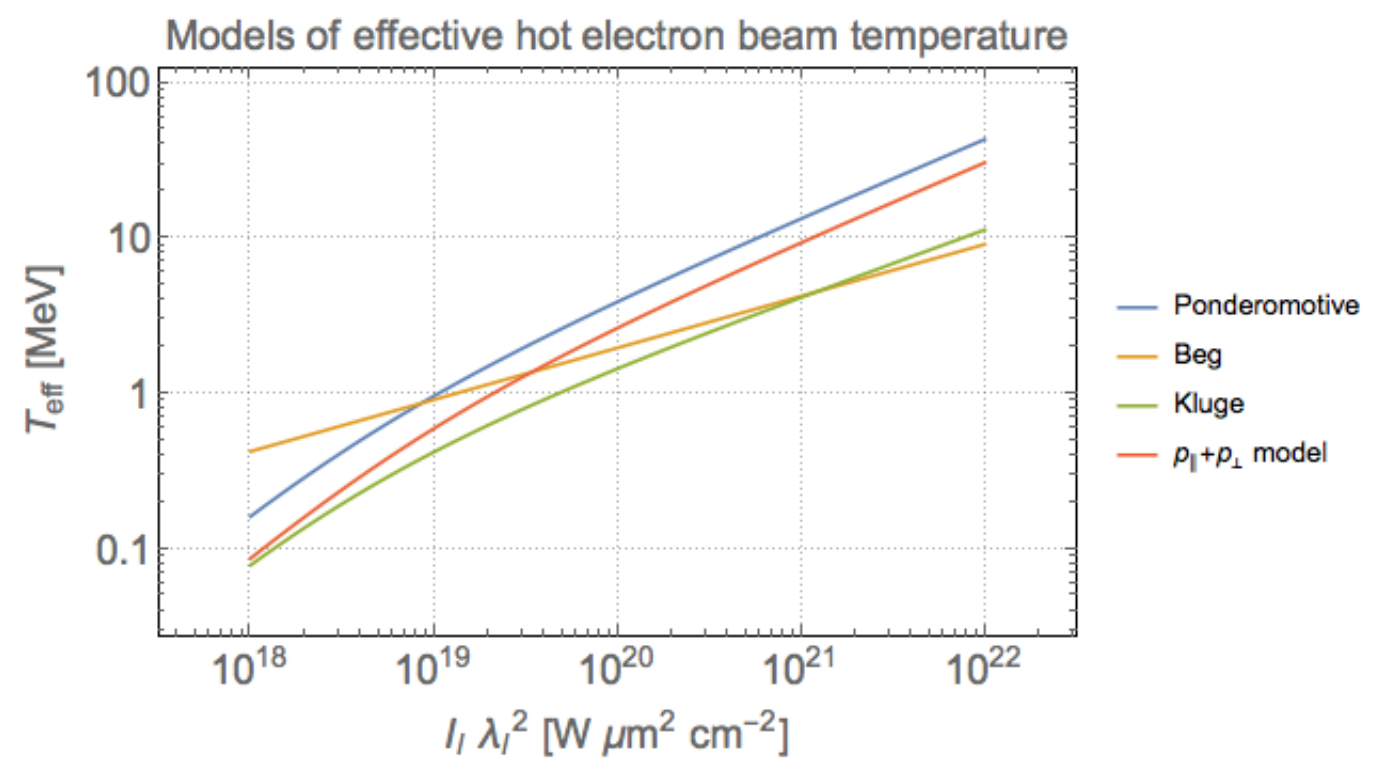

Figure 3.10 : Models of hot electron beam temperature including relativistic plane wave calculations (see text).

perature predictions are shown in Fig. 3.10. From this figure it is clear that the $\langle\gamma\rangle_{\text {Kluge }}$ model provides a good approximation of the Beg scaling, discussed in section 1.2.1, at higher intensities. This agreement suggests that the transverse Lorentz factor is appropriate in situations of very high laser contrast, in which electrons cannot effectively gain energy from the laser. The $\langle\gamma\rangle_{p \|+p \perp}$ model is coincident with the $\langle\gamma\rangle_{\text {Kluge }}$ model at low intensity but diverges at high intensity. This can be understood in connection to the motion of a single electron in a plane wave. When the wave field is weak, the electron transverse momentum is much larger than its longitudinal momentum. Yet as the wave becomes increasingly relativistic, the electron momentum in the longitudinal direction becomes dominant. Fig. 3.10 shows that as the laser intensity increases, $\langle\gamma\rangle_{p \|+p \perp}$ increases much more rapidly than $\langle\gamma\rangle_{\text {Kluge. }}$. This result supports that the ponderomotive scaling applies to more realistic situations in which the vacuum-plasma interface is not perfectly steep, i.e., electrons can sample the 
wave phase more fully and gain energy effectively from the laser. Additional research will focus on elucidating aspects of the relativistic plane wave model in connection to electron acceleration mechanisms active in realistic petawatt laser-plasma situations.

\subsection{Petawatt laser absorption bounded}

Key metrics for the advanced scientific applications based on petawatt laser solid interactions described in section 1 relate to absorption, yet conditions in this regime are so nonlinear that it is often impossible to know the fraction of absorbed light $f$, and even the range of $f$ is unknown. In this section, based on the relativistic RankineHugoniot-like analysis developed in section 3.1, we show for the first time that $f$ exhibits a theoretical maximum and minimum. These bounds constrain nonlinear absorption mechanisms across the petawatt regime, forbidding high absorption values at low laser power and low absorption values at high laser power. For applications needing to circumvent the absorption bounds, these results will accelerate a shift from solid targets, towards structured and multilayer targets, and lead the development of new materials.

Over the past two decades, the promise of the applications described in section 1.1 has driven considerable theoretical and experimental study of the crucial problem of how the laser energy is converted to target particle energy. Dozens of energy transfer mechanisms have been identified, $[214,129,166]$ and most treatments to date have focused on examining individual mechanisms in isolation to help guide interpretation of results. In realistic situations, however, these absorption mechanisms can be strongly nonlinear and several often act concurrently. In this section we derive the theoretical maximum and minimum absorption for each laser-solid configuration across the petawatt regime. We find that these extrema constrain nonlinear ab- 
sorption mechanisms, $[214,24,95,150,84,140]$ bounding the laser energy transfer in a more general manner. The present analysis overcomes difficulties of particle nonlinearity by creating a kinematic basis on which to formulate the interaction. We use a geometry centered at the laser-matter interface, taking advantage of the laser decay into an evanescent wave over a relativistic collisionless skin depth in the optically-thick target. Here Rankine-Hugoniot-like conservation laws[206, 39] must be satisfied by the forward-going evanescent light wave, the backward-going reflected wave, and forward-drifting highly relativistic electrons and moderately relativistic ions accelerated by the laser. By representing the complex motion of individual particles with ensemble properties such as density and momentum, accounting for the relativistically-correct laser-solid physics, $[215,72,163,175,62,83,106]$ we realize an essentially four body kinematics situation. We show that these kinematics restrict values the ensemble properties of electrons and ions can take on. Since acceleration of electrons and acceleration of ions are modes of absorption of laser light, we demonstrate that these kinematic restrictions can be transformed into useful upper and lower bounds on absorption. Excellent agreement with a broad range of published experimental and simulation data[150, 163, 106, 37] confirms that the absorption bounds are distilling a fundamental aspect of the nonlinear dynamical physics. For applications using solid targets, our results show a new general metric for measuring efficiency. Since the design space to be explored is contracted, these findings will enable research efforts to focus on useful regions of parameter space thus accelerating the development of future laser-solid applications. We also identify applications requiring efficiency exceeding that permitted by the absorption bounds. Our results indicate that these applications would benefit by shifting towards structured $[223,80,89,167]$ or multilayer[198, 191] target designs. 


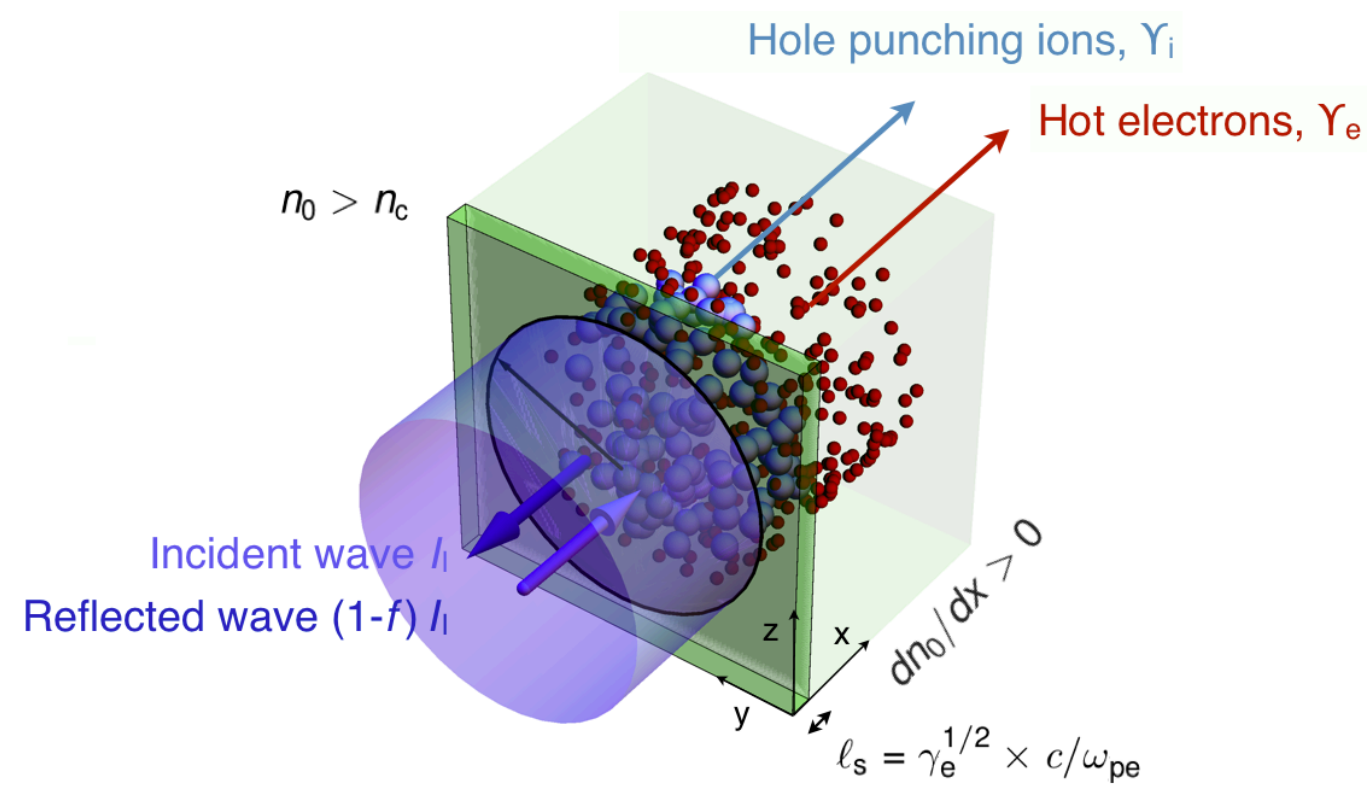

Figure 3.11 : Schematic showing key features of the petawatt laser-solid interaction. A high-power laser with strength parameter $a_{0}>1$ is shown striking an overdense target, interacting over the Lorentz-transformed collisionless skin depth $\ell_{\mathrm{s}}$ (dark green region), and exciting a highly-relativistic electron flux (red spheres) and moderatelyrelativistic ion flux (blue spheres). Laser and excited particle properties are connected across $\ell_{\mathrm{s}}$ by applying relativistic Rankine-Hugoniot-like relations at the laser-matter interface, allowing abstraction of downstream effects, e.g., scattering in the $x>\ell_{\mathrm{s}}$ target (light green region). Depiction uses a frame of reference co-moving with the interface. 


\subsubsection{Relativistic interaction model}

Essential features of petawatt laser-solid interactions are shown in Fig. 3.11. Here an ultraintense $a_{0}>1$ light pulse (where $a_{0}=e E_{1} /\left(m_{\mathrm{e}} c \omega_{1}\right)$ is the laser strength parameter, $c$ is the speed of light, $e$ is the fundamental charge, $m_{\mathrm{e}}$ is the electron mass, $E_{1}$ is the laser electric field and $\omega_{1}$ is the laser angular frequency) is seen to irradiate a thick target of electron density $n_{0}(x)>n_{\mathrm{c}}$, for realistic spatial profile $n_{0}(x)$ and critical density $n_{\mathrm{c}}=m_{\mathrm{e}} \omega_{1}^{2} /\left(4 \pi e^{2}\right)$. Electrons oscillate relativistically in the intense laser fields allowing the light wave to penetrate into the field-ionized overdense (optically-thick) plasma[26] an axial distance equal to the Lorentz-transformed collisionless skin depth, $\ell_{\mathrm{s}}=\gamma_{\mathrm{e}}^{1 / 2} \times c / \omega_{\mathrm{pe}}\left(\right.$ where $\gamma_{\mathrm{e}}=\left(1-\beta_{\mathrm{e}}^{2}\right)^{-1 / 2}$ is the electron Lorentz factor, $\beta_{\mathrm{e}} c$ is the electron speed and $\omega_{\mathrm{pe}}=\left(4 \pi e^{2} n_{0} / m_{\mathrm{e}}\right)^{1 / 2}$ is the plasma frequency).

The $\gamma_{\mathrm{e}}^{1 / 2}$ dependence appears in $\ell_{\mathrm{s}}$ due to the relativistic mass increase of electrons $m_{e} \rightarrow \gamma_{\mathrm{e}} m_{e}$ and the fact that $\ell_{\mathrm{s}} \propto \omega_{\mathrm{pe}}^{-1} \propto \sqrt{m_{e}}$. This forms the scale size for the interaction. Within $\ell_{\mathrm{s}}$, radiation reaction effects are small due to Debye shielding and electron and ion collisional mean-free paths satisfy $\lambda_{\text {mfp }} \gg \ell_{\mathrm{s}}$. Therefore, as shown in Fig. 3.11, electrons and ions are the only particle populations entering into the petawatt-scale kinematic interaction. Irrespective of the specific mechanism of energy transfer, these particles absorb energy from the laser collisionlessly, $[215,40]$ and their ensemble properties enter into formulae describing the total absorption $f=f_{\mathrm{i}}+f_{\mathrm{e}}$, where $f_{\mathrm{i}}$ ( $f_{a}^{p}$ using the notation of section 3.1) is the absorption into ions and $f_{\mathrm{e}}\left(f_{a}^{h}\right)$ is the absorption into electrons.

Unbounded $f_{\mathrm{e}}$ and $f_{\mathrm{i}}$ solutions are obtained by applying a relativistic kinematic model at the laser-matter interface, establishing a connection between the laser pulse and the particles it excites across the density discontinuity[106] (realizing an essential similarity to the Rankine-Hugoniot relations in magnetohydrodynamic shocks[39]). 
The essential kinematic relations forming the basis of the optimization analysis reported in this article were published in Ref. [106] and reviewed in section 3.1. The general consideration of optimal couplings under the constraint of phase space conservation motivates the present studies.[52] Radiation-hydrodynamic simulations show that particle density in interactions can often be approximated by an exponential distribution $n_{0}(x) \propto e^{-x / \ell_{\mathrm{p}}}$ for scalelength $\ell_{\mathrm{p}}$, due to amplified spontaneous emission (ASE) associated with laser pulse compression generating a 'pre-plasma.' [129] Petawatt laser-solids satisfy $\ell_{\mathrm{p}}[\mu \mathrm{m}]<1.1 a_{0} \tau_{1}[\mathrm{ps}]$ for pulse duration $\tau_{1}$ such that the primary interaction occurs in the classically-overdense $n_{0} / n_{\mathrm{c}}>1$ region while small-scale underdense regions are swept away by the strong laser ponderomotive force, as indicated by energy balance between electron acceleration in the underdense and overdense regions. The laser temporal envelope $I_{1}\left(\partial I_{1} / \partial t\right)^{-1} \gg 2 \pi \omega_{\mathrm{pe}}^{-1}$ and the plasma density profile is subject to $n_{0}\left(\partial n_{0} / \partial x\right)^{-1} \gg \ell_{\mathrm{s}}$, both readily satisfied under realistic conditions. Damping of transient momentum effects requires that $\tau_{1} \omega_{\mathrm{pi}}>2 \pi A$ where $\omega_{\mathrm{pi}}=\left(4 \pi e^{2} Z n_{0} / M_{\mathrm{i}}\right)^{1 / 2}$ and $A \simeq 3-5$, and the target thickness $d$ should exceed the hole punching depth and the effective refluxing hot electron range, $d>c \tau_{1} / 2+\int_{0}^{\tau_{1}} V_{\text {int }} d t$ for motion of the laser-matter interface at velocity $V_{\text {int }}$. Deviations from $\beta_{\mathbf{e}} \cdot \hat{\mathbf{k}}_{\mathbf{l}} \approx 1$ are second order in the angle $\tan ^{-1}\left[\left|\beta_{\mathbf{e}} \times \hat{\mathbf{k}}_{\mathbf{l}}\right| / \beta_{\mathbf{e}} \cdot \hat{\mathbf{k}}_{\mathbf{l}}\right]$ and therefore do not substantively affect the absorption bound results for realistic scenarios. Energy apportionment into ions increases with this angle but qualitative trends in $f_{\mathrm{e}}$ and $f_{\mathrm{i}}$ are maintained.[107] The analysis presented in section 1.2.4 plays an essential role in confirming the selection of absorption modes characterizing the petawatt-scale interaction, and shows a path to extending the absorption model to the 10-petawatt scale.

Ion dynamics are constrained by a snow plow-like process called 'hole punching' driven by the laser ponderomotive pressure, which can exceed $>10^{9}$ atmospheres. [215] 
Electron dynamics, on the other hand, can be governed by a number of different collisionless mechanisms depending on parameters such as laser polarization and angle of incidence.[214, 24, 95, 150, 84, 140] In order to calculate results independent of the specific mechanism, ensemble electron properties are determined based on a general Lorentz-invariant ansatz distribution function. [92] Solutions accounting for these realistic dynamical conditions are computed numerically, however an analytic form exists for the representative case $\beta_{\mathbf{e}} \cdot \hat{\mathbf{k}}_{\mathbf{l}} \approx 1$ for laser propagation in $\hat{\mathbf{k}}_{\mathbf{l}}$. Here ion absorption is $f_{\mathrm{i}}=2 \beta_{0} \mathcal{R}^{3 / 2} /\left[\sqrt{\beta_{0}^{2} \mathcal{R}+1}-\beta_{0} \sqrt{\mathcal{R}}\right]$ and electron absorption is $f_{\mathrm{e}}=\left[(1-\mathcal{R}) \sqrt{\beta_{0}^{2} \mathcal{R}+1}-(1+\mathcal{R}) \beta_{0} \sqrt{\mathcal{R}}\right] /\left[\sqrt{\beta_{0}^{2} \mathcal{R}+1}-\beta_{0} \sqrt{\mathcal{R}}\right]+O\left(\bar{\rho}_{h} / \beta_{0}^{2}\right)$, as given by equations (3.40) and (3.41) in section 3.1, using the convenient control parameters $\beta_{0}, \mathcal{R}$ and $\bar{\rho}_{h}$. Intensity and density conditions are controlled by $\beta_{0}=\left[Z m_{\mathrm{e}} n_{\mathrm{c}} /\left(2 M_{\mathrm{i}} n_{0}\right)\right]^{1 / 2} a_{0}$ for uniform interface charge state $Z$ and ion mass $M_{\mathrm{i}}$. $\mathcal{R}=I_{1, \text { reflected }} / I_{1, \text { incident }}$ is associated with the net photon flux deposited in the lasermatter interface, and $\bar{\rho}_{h}=n_{\mathrm{e}} m_{\mathrm{e}} /\left[n_{\mathrm{i}}\left(M_{\mathrm{i}}+Z m_{\mathrm{e}}\right)\right] \ll 1$ is a small parameter exhibiting the disparate mass scales that characterize the petawatt laser-solid absorption modes.

\subsubsection{Absorption bounds}

Absorption bounds reflect the fact that solutions to the kinematic equations for $f_{\mathrm{e}}$ and $f_{\mathrm{i}}$ can become nonphysical for values of $f$ between zero and one. These bounds are derived using constrained optimization techniques[142] with $f=f_{\mathrm{i}}+f_{\mathrm{e}}$ as the objective function. We optimize $f$ over $\mathcal{R}$ imposing the simple constraint that the electron energy is real, and the minimization equation is written as $f_{*}=\operatorname{Min}\left(f_{\mathrm{e}}+f_{\mathrm{i}}\right)$, s.t. $\gamma_{\mathrm{e}} \geq$ 1. Because the utility function is nonlinear in the control variables, minimization is performed numerically by means of cylindrical algebraic decomposition[33], and the resulting points are fit to a polynomial using interval $\Delta \beta_{0} \approx 10^{-3}$ over the 


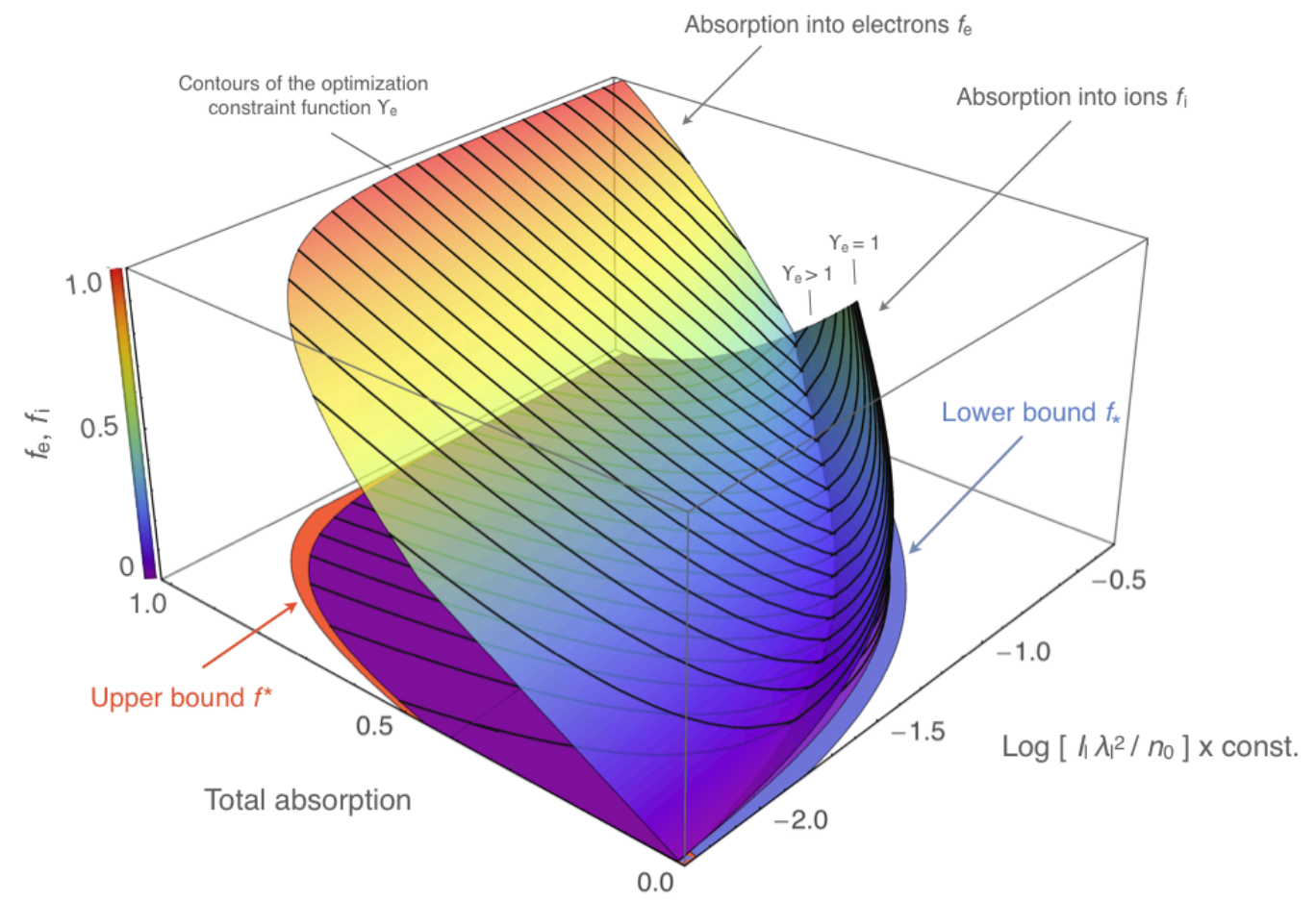

Figure 3.12 : Bounds on absorption and sub-partitioning of absorbed light. The lower-right axis corresponds to $\beta_{0}$ describing the initial laser and target conditions, and the total absorption $f=1-\mathcal{R}$. Two surfaces corresponding to the absorption into electrons $f_{\mathrm{e}}$ and into ions $f_{\mathrm{i}}$ are shown (the former having slight transparency for visualization purposes). Contours of the optimization function $\gamma_{\mathrm{e}}$ are superimposed on these surfaces using dark gray. The lower limit $f_{*}$ (blue) and upper limit $f^{*}$ (red) on absorption are shown bounding $f_{\mathrm{e}}$ and $f_{\mathrm{i}}$. 
physically-relevant range in $\beta_{0}$ between $[0.01,0.5]$. For the fully-ionized laser-plasma interface we calculate that $f_{*} \approx 1.9 \beta_{0}-2.75 \beta_{0}^{2}+1.91 \beta_{0}^{3}$, indicating that the lower limit on laser absorption is closely related to the process of ion acceleration by an intense circularly-polarized radiation pressure source[214, 150, 192]. Deviations from the absorption associated with this process occur at small $\beta_{0}$ as energy is reapportioned into relativistic electrons, highlighting that the kinematic coupling between ions and electrons represents an important feature of the interaction. When the electronic coupling is removed, we confirmed that $f_{*}$ converges to the well-established ion acceleration result $f_{*} \rightarrow 2 \beta_{0} /\left(1+2 \beta_{0}\right)$.[150] Maximizing the absorption through $f^{*}=\operatorname{Max}\left(f_{\mathrm{e}}+f_{\mathrm{i}}\right)$, again subject to the constraint that $\gamma_{\mathrm{e}} \geq 1$, computes the upper limit to be $f^{*}=1-\bar{\rho}_{h} /\left(2 \beta_{0}^{2}\right)$. In contrast to the lower limit, there is no wellestablished analytic result that describes absorption along the $f^{*}$ curve based on a simple physical mechanism. Here we proceed allowing that $n_{\mathrm{e}} \approx n_{\mathrm{c}}$, implying that

absorption along $f=f^{*}$ corresponds to electrons excited with $\gamma_{\mathrm{e}} \approx a_{0}^{2} / 2$, within a factor of order unity of the full laser ponderomotive potential. Fig. 3.12 presents a comprehensive description of the absorption, showing surfaces corresponding to $f_{\mathrm{e}}$ and $f_{\mathrm{i}}$, as well as bounding regions corresponding to $f_{*}$ and $f^{*}$. Ions are seen to dominate the absorption along $f=f_{*}$, the region corresponding to $\gamma_{\mathrm{e}}=1$. As the target absorbs more of the laser energy, Fig. 3.12 shows that this energy is predominantly coupled into relativistic electrons. Electrons dominate the absorption along $f=f^{*}$, with $f>f^{*}$ causing $f_{\mathrm{e}}$ to take on complex value.

\subsubsection{Comparison between absorption bounds and published data}

Comparison to published data is facilitated by specifying an interaction-averaged density, which is well-represented by a corrected relativistic critical density[26] given 


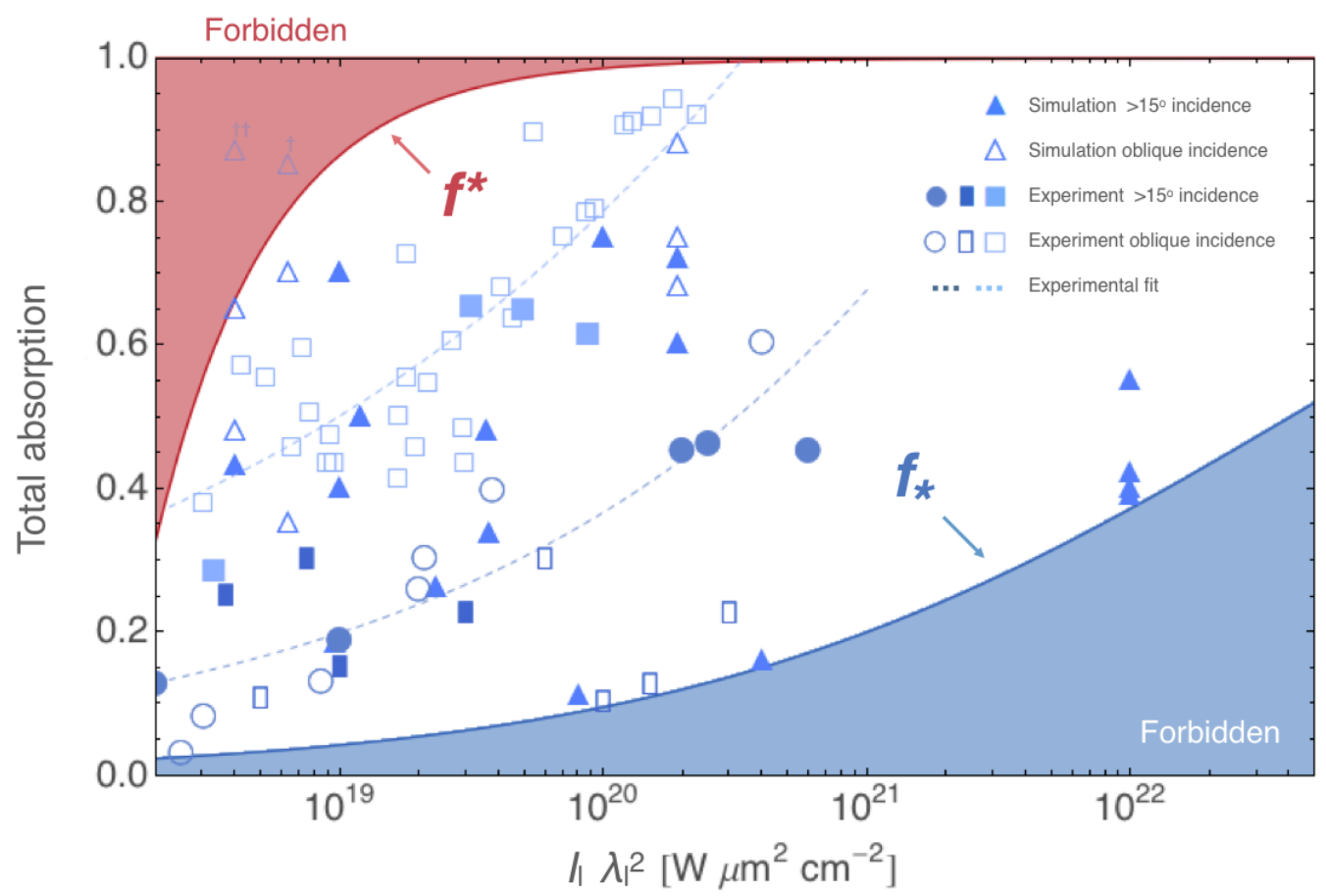

Figure 3.13 : Comparison between absorption bounds and published data. The complete dataset compiled in Davies [37] is reproduced here, spanning experimental and simulation data published over the past two decades, across a variety of laser and plasma conditions. Dashed lines corresponding to fits of selected experimental data are shown to guide the eye. Additional high-intensity simulation data is reproduced from Levy et al.[106] The upper limit on absorption $f^{*}$ is depicted in red and the lower limit $f_{*}$ in blue, with forbidden regions indicated using shading. The two outlying data points correspond to simulations of ${ }^{\dagger}$ ) a very thin $0.2 \mu \mathrm{m}$ pre-deformed target, and ${ }^{(\dagger \dagger)}$ an essentially underdense $n_{\mathrm{c}}>n_{0}$ interaction, both violating assumptions underpinning the laser-solid model. 
by $a_{0}^{2} \approx(27 / 64)\left(n_{0} / n_{\mathrm{c}}\right)^{4}$ for $n_{0} / n_{\mathrm{c}} \gg 1$. Fig. 3.13 shows these limits applied to experimental data and simulation results published over two decades, spanning a broad range of laser and plasma parameters, obtained at several laser facilities. From this figure it is clear that experimental data and kinetic particle-in-cell simulations at a variety of realistic conditions $[150,163,106,37]$ show excellent agreement with the absorption bound predictions.

\subsubsection{Absorption bounds in terms of laser and plasma parameters}

Transforming from the control coordinates, the condition $f_{*} \leq f \leq f^{*}$ can be written simply in terms of the laser power and unperturbed plasma density as,

$$
\frac{\sqrt{I_{1} \lambda_{1}^{2}}}{\sqrt{I_{1} \lambda_{1}^{2}}+1.5 \sqrt{n_{0}}} \leq f \leq 1-\frac{1.2 \times 10^{18}}{I_{1} \lambda_{1}^{2}}
$$

where $\left[\begin{array}{ll}I_{1} & \lambda_{\mathrm{l}}^{2}\end{array}\right]=\mathrm{W} \mu \mathrm{m}^{2} \mathrm{~cm}^{-2},\left[n_{0}\right]=\mathrm{cm}^{-3}$, and $I_{1} \lambda_{l}^{2}>1.3 \times 10^{18}$. Equation (3.72) bounds the laser-solid interaction through its dynamical history for a realistic timedependent laser envelope and plasma profile.[150, 106]

Fig. 3.12 and 3.13 highlight that $f_{*}$ becomes increasingly strict with laser power, forbidding $35 \%$ of possible absorption values at $I_{1} \lambda_{1}^{2} \sim 10^{22} \mathrm{~W} \mu \mathrm{m}^{2} \mathrm{~cm}^{-2}$, a regime accessible at laser facilities such as ELI[1] scheduled to come online in the next few years. These results will therefore play a central role in guiding the next generation of multi-petawatt experiments.

For applications needing to circumvent the absorption bounds in equation (3.72), these results will drive a shift towards new interaction paradigms. In order to see that assumptions underpinning the laser-solid interaction model must be violated in order to exceed these limits it is instructive to examine the two outlying points 
shown in Fig. 3.13. The data point labeled ${ }^{(\dagger)}$ corresponds to one simulation of a pre-deformed, very thin target of $\lambda_{\mathrm{l}}>d$ where $d$ is the target thickness, realizing a strongly refluxing configuration. The point labeled ${ }^{(\dagger \dagger)}$ corresponds to one simulation of a laser interacting with $20 \mu \mathrm{m}$ of $n_{\mathrm{c}}>n_{0}$ plasma in front of a thin $n_{0}=20 n_{\mathrm{c}}$ target, realizing an essentially underdense situation. We thus confirm that very thin and underdense targets allow absorption in excess of $f^{*}$ at low laser power, as they should. However, several important applications that have conventionally used solid targets also depend on high absorption at relatively low laser power. These applications include laser-based anti-matter generation for scaled astrophysical studies,[121, 31] ultrafast charged-particle imaging systems,[216] where increasing absorption reduces noise and improves imaging resolution, and certain approaches to electron-driven fast ignition laser fusion.[204] Recently works that have shifted from solid targets have started to report enhanced results in these areas.[167, 198, 191] The results presented here will accelerate this shift from solid targets across the petawatt field, and lead the development of novel low density, structured and multilayer targets.

\subsection{Phase space accessible to the petawatt laser-plasma sys- tem}

In this section we examine the phase space constraints on the ultraintense laser-solid system, accounting for both the oscillatory (high-frequency) and steady-state absorption modes. As shown in section 3.5 applying constrained minimization techniques[142], to the objective function $f$ yields theoretical upper and lower bounds on absorption. The limits are sensitive to hot electrons properties at low intensity and dominated by ions at high intensity.

In this section we derive a quantitative measure for the degree of constraint as- 
sociated with the laser-plasma interaction. The preceding analysis showed that to zeroth order in $\bar{\rho}_{h}$ the kinematic absorption efficiencies are fully-parametrized by the variables $\left(\beta_{\mathbf{0}}, \mathcal{R}\right)$. In terms of these variables, the differential phase space element associated with a $1-D$ interaction is simply $\mathrm{d} \Omega=\mathrm{d} \mathcal{R} \mathrm{d} \beta_{0}$. The fraction of phase volume accessible to the laser-plasma interaction, $\Lambda$, is thus given by,

$$
\Lambda \equiv \frac{\iint_{1-f_{*}}^{1-f^{*}} \mathrm{~d} \mathcal{R} \mathrm{d} \beta_{0}}{\int \mathrm{d} \Omega}
$$

where the selection of a proper overdense target is assumed. The integral bounds in equation (3.73) shed light on the essential role played by hot electrons in the phase space analysis, across all intensities, allowing the system to access states lying below $1-f_{*}$. Without the additional electronic degree of freedom, i.e., in the ion-only system, the phase volume of solutions collapses to a single point.

The accessible phase space volume $\Lambda$ can be evaluated exactly in the case of $f^{*} \rightarrow 1$ as,

$$
\Lambda=\frac{\ln \left(1+2 \beta_{0}\right)}{2 \beta_{0}}
$$

Accounting for the finite upper limit on absorption $f^{*}$ requires that we select bounds on $\beta_{0}$ such that that the integral converges, since $f^{*} \propto \beta_{0}^{-2}$. In order to satisfy this condition we adopt the lower integral bound of $\beta_{0}=0.01$, yielding,

$$
\Lambda=-\frac{50}{\beta_{0}} \frac{\beta_{0} \ln (51 / 50)-\beta_{0} \ln \left(1+2 \beta_{0}\right)+\left(100 \beta_{0}-1\right) \bar{\rho}_{h}}{100 \beta_{0}-1}
$$

for the limiting case of $\bar{\rho}_{h}=m_{e} /\left(2 m_{p}\right) \equiv \rho_{h 0}$ (where $\left.m_{p} / m_{e}=1836\right)$. Fig. 3.14 depicts $\Lambda$ as a function of the parameter $\beta_{0}$ describing the unperturbed plasma density 


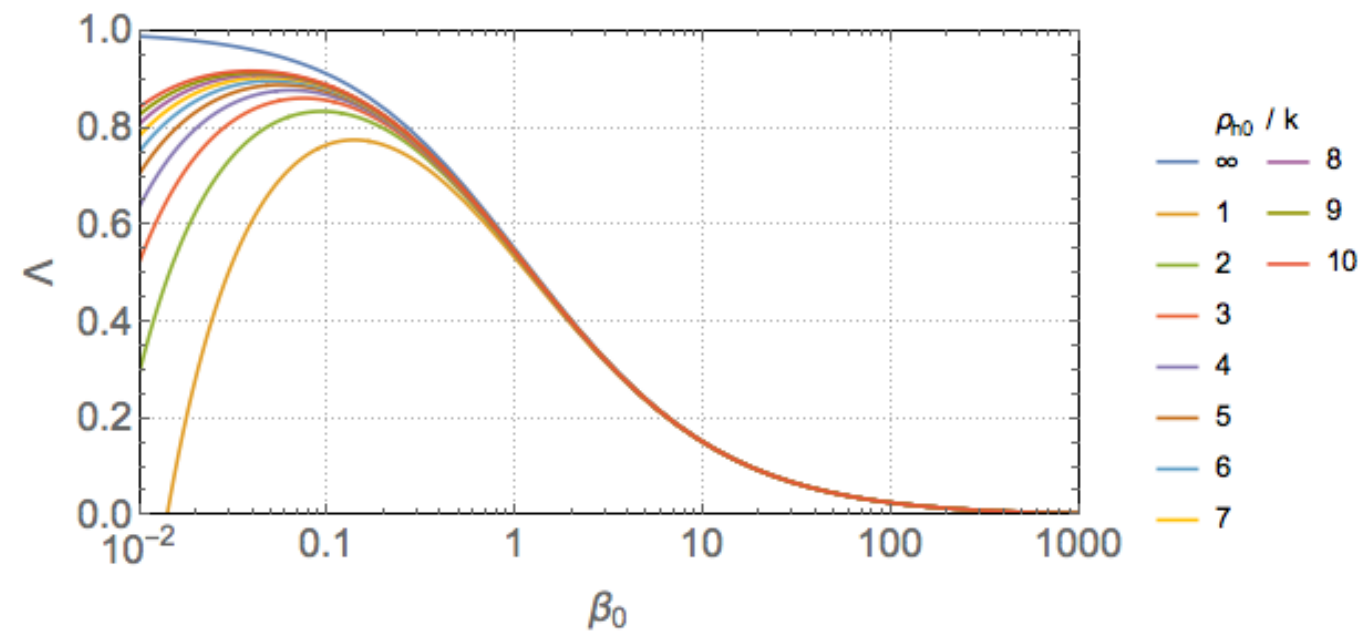

Figure 3.14 : Fraction of phase space accessible to the petawatt laser-plasma system, $\Lambda$ (see text).

and normalized laser intensity $a_{0}$, where we have exploited the relativistic nature of the model to consider cases extending out to large $\beta_{0}$. The curves correspond to values of $\rho_{h 0} / k$, with $k=\infty$ corresponding to equation (3.74).

The $\Lambda$ curves shown in Fig. 3.14 suggest interesting features of the laser-plasma system. First we notice that when $k<\infty$ that $\Lambda \rightarrow 0$ for small values of $\beta_{0}$. This region corresponds to small $a_{0}$, i.e., to cases near the threshold for relativistic electron motion in the laser fields, as discussed in sections surrounding equation (1.2). Indeed, the absorption model breaks down at this point since the low collisionality assumptions underpinning it are violated. That $\Lambda \approx 0$ implies that alternative modes of absorption play an important role in this regime, and indeed the dominance of these mechanisms, e.g., inverse bremsstrahlung[214], for $a_{0}<1$ is already well known. In principle the petawatt-scale absorption model can be extended to include these effects; this possibility will be assayed in future research. For certain values of $\bar{\rho}_{h}$ Fig. 3.14 


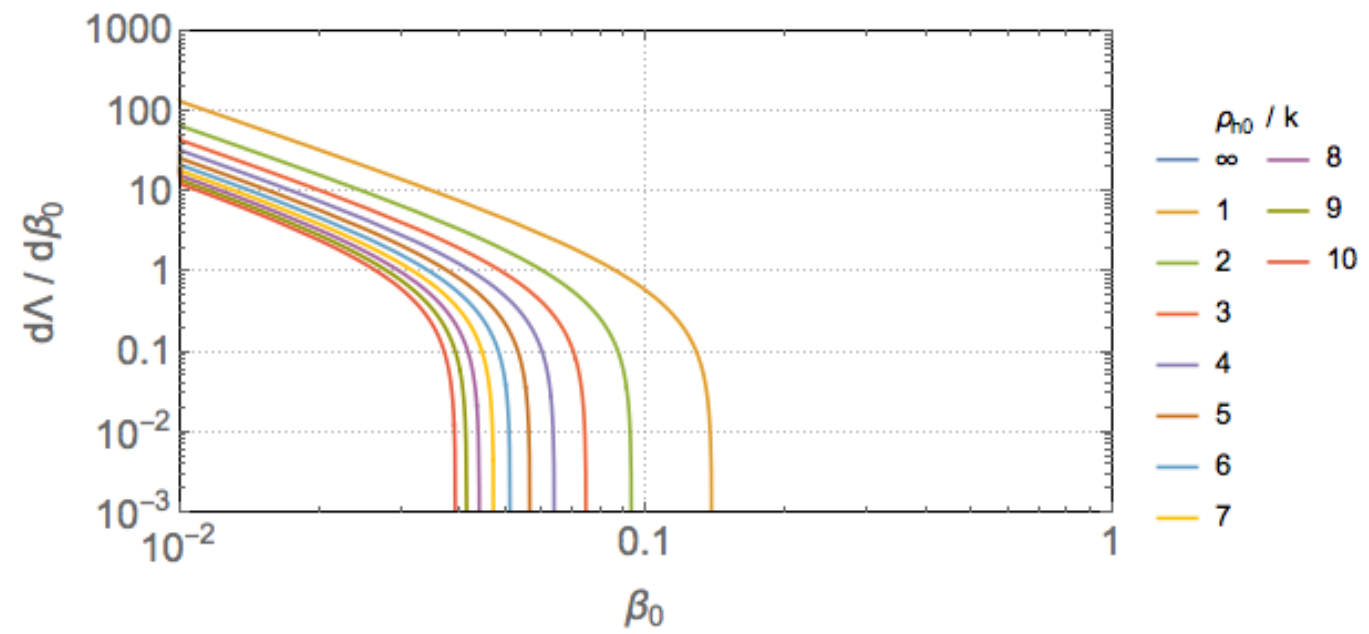

Figure 3.15 : Rate of change of the $\Lambda$ with $\beta_{0}$ indicating that the number of states accessible to the laser-plasma system is maximized for $\beta_{0} \sim 0.1$ (see text). Note different scales relative to Fig. 3.14.

shows that $\Lambda \approx 0$ even when $\beta_{0} \sim 0.01$ and $a_{0}>1$. This implies a bound on the number of hot electrons that can be excited in this regime, a notion that will be considered in detail in future work.

Another interesting aspect of $\Lambda$ manifests at intermediate values of $\beta_{0}$. As shown in Fig. 3.15, across the range of finite $k$ values, the value of $\Lambda$ is maximized at $\beta_{0} \sim 0.1$. To the extent that the fraction of phase space states accessible to the laser-plasma system correlates with its dynamical behavior, the emergence of unique aspects can be anticipated at this point. If $\Lambda \propto f$ an inflection point in the fractional laser energy absorbed by the plasma is predicted unambiguously. The laser energy $\mathcal{E}_{l} \propto a_{0}^{2}$ but in the ponderomotive scaling [215] the hot electron energy $\mathcal{E}_{e} \propto a_{0}$. Hence, if the hot electrons dominate the absorption, the expectation of an inflection point in $f$ is not physically unreasonable. Furthermore, we have shown in Fig. 1.14 that the onset for relativistic effects in the ion dynamics occurs as $\beta_{0} \sim 0.1-1$, coincident with the present region of interest. It is therefore possible that $d \Lambda / d \beta_{0} \sim 0$ correlates 
with the dominance of absorption into ions, on average, relative to electrons. Future research will refine these considerations.

Finally we comment on the significant reduction in $\Lambda$ at high values of $\beta_{0}$ as shown in Fig. 3.14. In the region $\beta_{0} \sim 1$, corresponding to strongly relativisticallydriven ions relevant to emerging-class laser systems such as ELI scheduled to come online in the next few years $[36,130,1]$, we observe that $\sim 50 \%$ of the phase space volume is restricted in the interaction. The related contraction in allowed values of $f$ was discussed in section 3.5; the constriction of both $f$ and $\Lambda$ has important implications for the next generation of laser experiments. At $\beta_{0}>1$ new absorption mechanisms arise, not captured by the petawatt-scale absorption model, that are quantum electrodynamical in nature. These mechanisms are discussed in section 1.2.4, and additional research will focus on extending the present model into the future 10-petawatt regime. 


\section{Chapter 4}

\section{Relativistic laser interactions with realistic heterogeneous plasmas}

\subsection{Accelerating piston action and plasma heating in high energy density laser-plasma interactions}

In the field of high-energy density physics (HEDP), lasers in both the nanosecond and picosecond regimes can drive conditions in the laboratory relevant to a broad range of astrophysical phenomena, including gamma-ray burst afterglows and supernova remnants. In the short-pulse regime, the strong light pressure (> Gbar) associated ultraintense lasers of intensity $I>10^{18} \mathrm{~W} / \mathrm{cm}^{2}$ plays a central role in many HEDP applications. Yet, the behavior of this nonlinear pressure mechanism is not well-understood at late time in the laser-plasma interaction. In this section, a more realistic treatment of the laser pressure 'hole boring' process is developed through analytical modeling and particle-in-cell simulations. A simple Liouville code capturing the phase space evolution of ponderomotively-driven ions is employed to distill effects related to plasma heating and ion bulk acceleration. Taking into account these effects, our results show that the evolution of the laser-target system encompasses ponderomotive expansion, equipartition, and quasi-isothermal expansion epochs. These results have implications for light piston-driven ion acceleration scenarios, and astrophysical applications where the efficiencies of converting incident Poynting flux into bulk plasma flow and plasma heat are key unknown parameters. 
In particular, here we focus on the ability of the laser to heat the plasma and drive bulk plasma flows as it bores into the target. These are core unknowns in astrophysical jets and winds. There is growing enthusiasm among astrophysicists for the paradigm that Poynting flux-dominated outflows in gamma-ray bursts (e.g., [126]) and blazars drive their energization and dissipation at large distances from their central 'engines.' Understanding the efficiency of conversion of direct electromagnetic energy to plasma kinetic and thermal energies is an extremely desirable advance. This study is a step in this direction, anticipating that down the line, higher laser intensities will extend the focus to relativistic flow speeds germane to GRBs and blazars, precipitate rampant pair production, and therefore encapsulate the domain of pair jets, also pertinent to GRBs and blazars. Moreover, multiple laser-plasma interaction sites will establish an array of bulk flows, and these will in turn interact forming collisionless shock zones, where charges will be energized and radiate to generate the electromagnetic signals that we detect from these topical cosmic sources.

In this section, we develop a more realistic treatment of the ponderomotive hole boring process that results in qualitative modifications to the evolution of the lasertarget system, taking into account effects such as a realistic laser pulse profile and target-front plasma volumetric heating. A limit on the ponderomotive regime is identified at late time due to heating of plasma at the laser interface. There has been interest in this area recently both for equilibrium[104] and non-equilibrium configurations. For the latter, recent experimental work has observed this effect by measuring the doppler motion of the critical interface, showing a $1.5 \%\left(\Delta \lambda / \lambda_{0}\right)$ blue-shift at late time[162]. These thermal effects are considered here using a simple model and are seen to qualitatively modify the system dynamics while the laser driver remains strong. A Liouville code is developed to highlight and understand key aspects of the ponderomotive and the volumetric heating dynamics. Related to the former, it is 
observed that a fraction of the ion population is swept up and accelerated by the light piston multiple times. In many respects, our results show that the ponderomotive hole boring of a realistic laser pulse gives rise to richer particle dynamics, though ultimately represents a less robust 'pressure source' than previously thought.

\subsubsection{Development of a self-consistent framework for equations of motion}

We adopt the basics of the simple analytical model from Wilks et al. [215] and its modern interpretation by Silva and others[199, 131, 150] as a propagating 'double layer.' The structure is comprised of electrons accelerated by the laser fields to encompass spatially extended regions where the overdense plasma damps the laser electric field. Ions are pulled along through the generation of an ambipolar force field, with the structure propagating into the bulk target. We employ a simple force balance/hydrodynamic model to determine the effective temperature $T_{e}$ of the plasma interface over time. In this model we consider the response of ion particles in the presence of an electric field and a background effective fluid pressure, realizing a similarity to the particle/fluid equations of motion of hybrid particle-in-cell codes such as LSP[211]. For such particles in the laboratory frame the ion equation of motion is given by,

$$
\frac{d p_{i}}{d t}=e E+\frac{1}{n_{i}} \frac{\partial P_{i}}{\partial x}
$$

for momentum $p_{i}$, number density $n_{i}$, and pressure $P_{i}$ of the ion fluid. Here the components of these vector quantities are explicitly taken in the $x$-direction, namely the direction of laser propagation. Electrons in the interface experience forces related to the laser driver and to the effective plasma pressure, the effects of which couple to the ions through the generation of quasi-electrostatic fields. In our simple model, these forces are decoupled and act antiparallel to one another. The two equations of 
motion are given by

$$
\begin{aligned}
\frac{d}{d t} p_{e, L} & =e E-\frac{\partial}{\partial x}\left[\frac{m_{e} a_{0}^{2} c^{2} \omega_{L}^{2}}{\omega_{p e}^{2}} e^{-2 \omega_{p e} x / c}\left(1+\cos 2 \omega_{L} t\right)\right] \\
\frac{d}{d t} p_{e, t h} & =e E+\frac{1}{n_{e}} \frac{\partial}{\partial x}\left(n_{e} T_{e}\right)
\end{aligned}
$$

for the forces associated with the classical laser driver [214] and with the plasma pressure, respectively. Here $a_{0}=e E_{L} /\left(m_{e} \omega_{L} c\right)$ is the dimensionless laser parameter for an electric field amplitude $E_{L}$ of the laser. The first of these equations contains a gradient whose argument includes the laser intensity. By taking the time average of electron equations and neglecting terms with $m_{e}$ due to the small mass fraction we obtain,

$$
\begin{aligned}
\frac{d}{d t} p_{i, L} & =\frac{d}{d t}\left(\frac{I M_{i}}{n_{i} c}\right)^{1 / 2} \\
\frac{d}{d t} p_{i, t h} & =-\frac{d}{d t}\left(T_{e} M_{i}\right)^{1 / 2}
\end{aligned}
$$

Here we have assumed $Z / A=1 / 2$, appropriate for elements heavier than helium and lighter than iron and readily satisfied near the critical surface, and the ion pressure term $P_{i}=n_{i} T_{i}$ has been neglected as it is small compared to the electron pressure because $T_{e} / T_{i} \gg 1$.

The net ion motion in the critical interface is then obtained by summing the forces from Equation (4.3). In the nonrelativistic limit, the ion velocity may be solved through $u_{i}=M_{i}^{-1} \int(d / d t) p_{i, j} d t$, summing over $j \in\{L, t h\}$. Labeling this the 
ion front velocity $u_{f}$, we may write,

$$
u_{f}(x, t)=\left[\frac{I(t, x)}{M_{i} n_{i}(x) c}\right]^{1 / 2}-\left(\frac{T_{e}(t)}{M_{i}}\right)^{1 / 2}
$$

where the time and space-dependent profiles of the the laser and plasma quantities have been implicitly introduced.

As noted above, the second term on the right side of equation (5.4) corresponds to an effective temperature for electrons in the critical surface, reflecting contributions from various electron and ion populations that interact with the interface over time. During laser irradiation, there is a population of hot electrons, accelerated to the laser ponderomotive potential reaching multi-MeV, that passes through the interface rapidly, heating a background population of electrons resistively. The initially-cold background also experiences laser-parallel excursion into the target due to the timeaveraged component of the ponderomotive force, carrying along ions through the generation of an ambipolar field. In addition, laser-coherent relativistically oscillating electrons are present in the critical interface region. A fraction of these may de-phase over time with the laser, resulting in a residual non-adiabatic heating of the bulk. The interplay between these populations, each with their own spatio-temporal-dependent densities and energy scales, renders $T_{e}$ an approximation to the physical processes involved.

The temporal evolution of $u_{f}$ through equation (5.4) is depicted schematically in Fig. 4.6. At early time, the plasma expands with $u_{f}<0$ at a rate determined by bulk heating associated with the amplified spontaneous emission (ASE)-driven preplasma. As the ultraintense laser impinges upon the target, it propagates through the underdense plasma until reaching a surface with density $\gamma(t) n_{c r}$, where $\gamma=\left(1+a_{0}^{2}\right)^{1 / 2}$ is related to the relativistic plasma frequency damping. $u_{f}$ then increases rapidly as 
momentum flux is conserved in the interaction between the laser and ions in the critical interface. This corresponds to the start of the ponderomotive regime, labeled (I) in Fig. 4.6. Two additional unique dynamical regimes may be identified from equation (5.4), which we define in terms of a dimensionless thermal parameter $\Gamma(t) \equiv$ $I /\left(n_{i} c T_{e}\right)$, by analogy to the magnetic $\beta$ parameter. As depicted in Fig. 4.6, along with the ponderomotive regime defined by $\Gamma \gg 1$, these are the (II) equipartition regime where thermal effects become non-negligible with $\Gamma \simeq 1$; and the (III) quasiisothermal expansion regime, defined by $\Gamma \simeq 0$, where the laser driver has diminished and the heated plasma expands. With this framework in place, we will go about treating these regimes in chronological order.

\subsubsection{Analytical derivation of the front velocity for a realistic pulse en- velope in the ponderomotive regime}

In the ponderomotive regime, to address the effect on the system from the accelerating laser piston it is intuitive that we should first cast equation (5.4) as a function of time only. The ion plasma density is given by $n_{i}(x, t)=n_{0}(x)+\delta n(x, t)$. The former term represents the initial plasma density and latter term describes the dynamics related to ions accelerated by the laser piston at earlier time. For most common scenarios, because $\delta n / n_{0} \ll 1$ the latter does not strongly affect the piston motion. However, these ions may be swept up by the accelerating light piston multiple times, and we will see shortly that it is predominantly $\delta n$ contributions that qualitatively modify the ion phase space.

By noting the laser front location $x_{f}(t)=\int_{0}^{t} u_{f}\left(t^{\prime}\right) d t^{\prime}$, we have the total instantaneous plasma density at the piston, $n_{i}(t)=n_{0}\left(x_{f}\right)+\delta n\left(x_{f}, t\right)$. Equation (5.4) may 


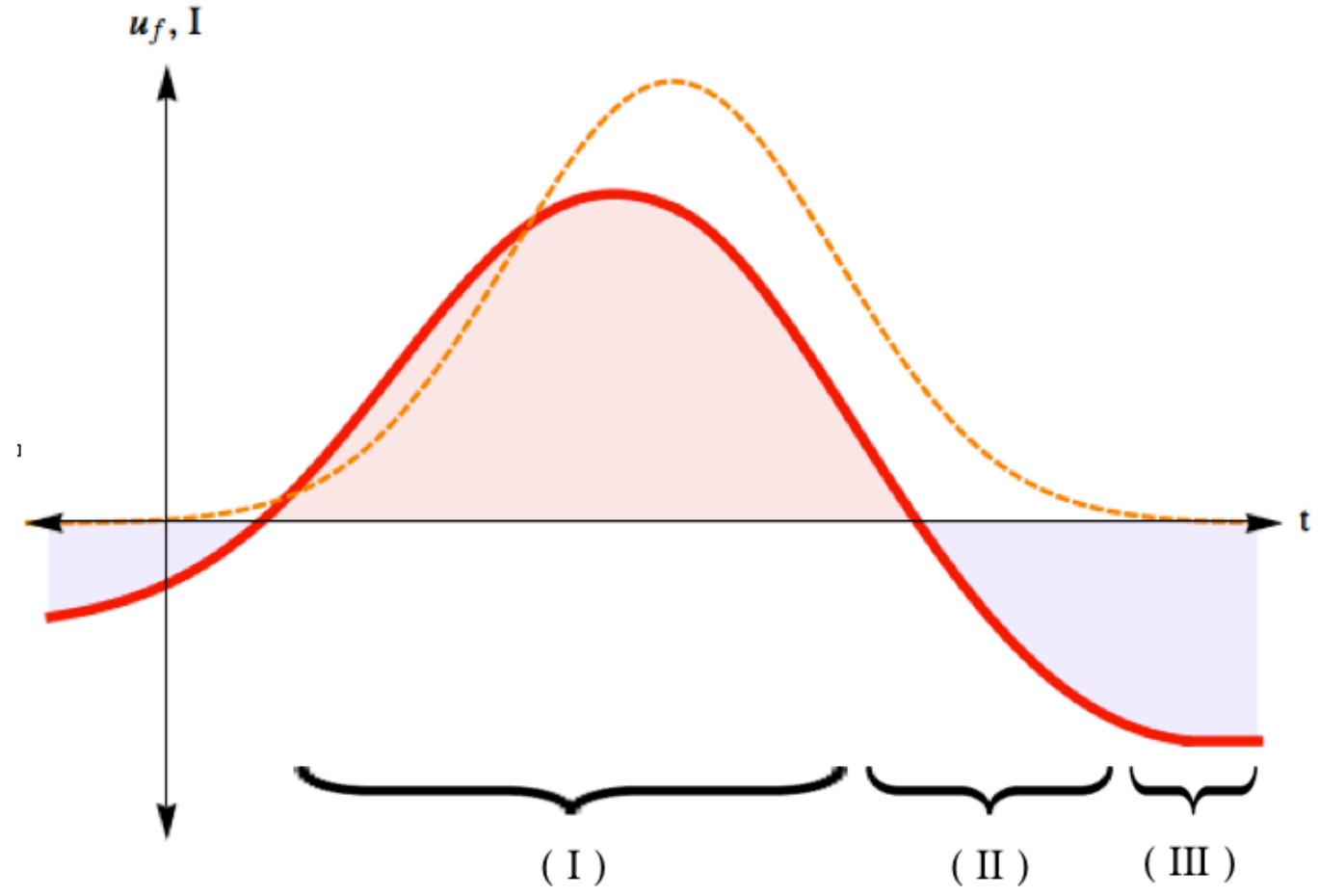

Figure 4.1 : Schematic depicting the temporal evolution of the front velocity $u_{f}$ (red) for Gaussian temporal laser intensity $I(t, x)$ (dashed orange) incident on an exponential plasma density profile. Shading below the $u_{f}$ curve represents the doppler shifted light reflected from the critical interface. Initially the plasma expands with $u_{f}<0$ at a rate determined by bulk heating associated with laser prepulse. $u_{f}$ is then seen to evolve through three distinct dynamical regimes over time, denoted by I, II and III (see text). 
then be rewritten as

$$
u_{f}(t)=\left[\frac{I(t)}{M_{i} c}\right]^{1 / 2}\left\{n_{0}\left(x_{f}(t)\right)+\delta n\left(x_{f}(t), t\right)\right\}^{-1 / 2}-\left[\frac{T_{e}(t)}{M_{i}}\right]^{1 / 2}
$$

In general, this equation for the laser hole boring velocity must be solved numerically. However, in the ponderomotive regime where $\Gamma \gg 1$, analytical solutions for many common scenarios may be obtained explicitly.

For a semi-infinite pulse of intensity $I_{0}$ incident on a steep plasma slab of density $n_{0}$, equation (4.5) recovers the ponderomotive velocity[215] as desired. For a semiinfinite pulse of intensity $I_{0}$ incident upon an exponential plasma density gradient, $n_{0}(x)=n_{0} \exp \left[\ell^{-1}\left(x-x_{s}\right)\right]$ of scalelength $\ell=n(d n / d x)^{-1}$, we employ separation of variables to solve for the front motion,

$$
\begin{aligned}
x_{f}(t) & =2 \ell \ln \left[1+e^{x_{s} /(2 \ell)} \frac{u_{w 0}}{2 \ell} t\right] \\
u_{f}(t) \equiv \frac{d x_{f}(t)}{d t} & =u_{w 0} e^{x_{s} /(2 \ell)}\left[1+e^{x_{s} /(2 \ell)} \frac{u_{w 0}}{2 \ell} t\right]^{-1}
\end{aligned}
$$

where $u_{w 0} \equiv\left[I_{0} /\left(M_{i} n_{0} c\right)\right]^{1 / 2}=\left[m_{e} n_{c r} a_{0}^{2} c^{2} /\left(2 M_{i} n_{p e 0}\right)\right]^{1 / 2}$ is the constant, characteristic ponderomotive velocity for the system. Here $n_{c r}=m_{e} \omega_{L}^{2} /\left(4 \pi e^{2}\right)$ is the critical density at which $\omega_{L} / \omega_{p e}=1$. The position $x_{c r}$ of the relativistic critical surface is given by the condition $n\left(x_{c r}\right)\left(\gamma n_{c r}\right)^{-1}=1$.

Now let us consider a more realistic temporal pulse profile, here a Gaussian of the form $I(t)=I_{0}(t) \exp \left[-2\left(t-\alpha t_{1 / 2}\right)^{2} / t_{1 / 2}^{2}\right]$ where $\alpha$ determines $I\left(t_{0}\right)$, and $t_{1 / 2}$ fixes the width of the pulse. This again will interact with an exponential plasma profile $n_{0}(x)=n_{0} \exp \left[\ell^{-1}\left(x-x_{s}\right)\right]$ of scalelength $\ell$. Assuming constant $I_{0}$, a closed-form 
solution may be obtained:

$$
x_{f}(t)=2 \ell \ln [\Lambda(t, x)] \quad, \quad u_{f}(t)=\frac{u_{w 0} e^{x_{s} /(2 \ell)}}{\Lambda(t, x)} e^{-\left(t-\alpha t_{1 / 2}\right)^{2} / t_{1 / 2}^{2}}
$$

where

$$
\Lambda(t, x)=1+e^{x_{s} /(2 \ell)} \frac{\sqrt{\pi} u_{w 0} t_{1 / 2}}{4 \ell} \Omega(t)
$$

for $\Omega(t) \equiv \operatorname{erf}(\alpha)+\operatorname{erf}\left[\left(t-\alpha t_{1 / 2}\right) / t_{1 / 2}\right]$, where $\operatorname{erf}(x)$ is the error function. Equations (4.6) and (4.7) describe qualitatively distinct dynamics of the light piston, as is depicted in Fig. 4.7 (A). For Gaussian pulses, the peak front velocity is seen to shift towards the peak laser drive time as intensity decreases. Fig. 4.7 (B) shows the effects of a realistic pulse envelope on the hole boring penetration distance. The reduction in channelling distance at $3 p s, \Delta x_{f} / x_{f}=\{0.30,0.27,0.22\}$ for pulses of intensity $\log I_{0}=\{19,20,21\}$, respectively. Under these system conditions, for a realistic pulse to bore in as far as a semi-infinite pulse, a factor of $\sim 2$ times more energy is required. This reduction in channeling efficiency has implications for the energy required for the short pulse laser beam in hole boring FI scenarios[204, 150]. In addition to determining the evolution of the target-front, these modifications to the front velocity also give rise to a novel richness in ion phase space; this will be treated shortly.

\subsubsection{Consideration of simple closure models for the effective electron temperature}

Let us consider the equipartition and isothermal expansion dynamical regimes, $\Gamma \lesssim 1$. Equation (4.5) describes the time evolution of the system, but to obtain a more complete picture we require a closure relation for $T_{e}$. By balancing momentum flux when 


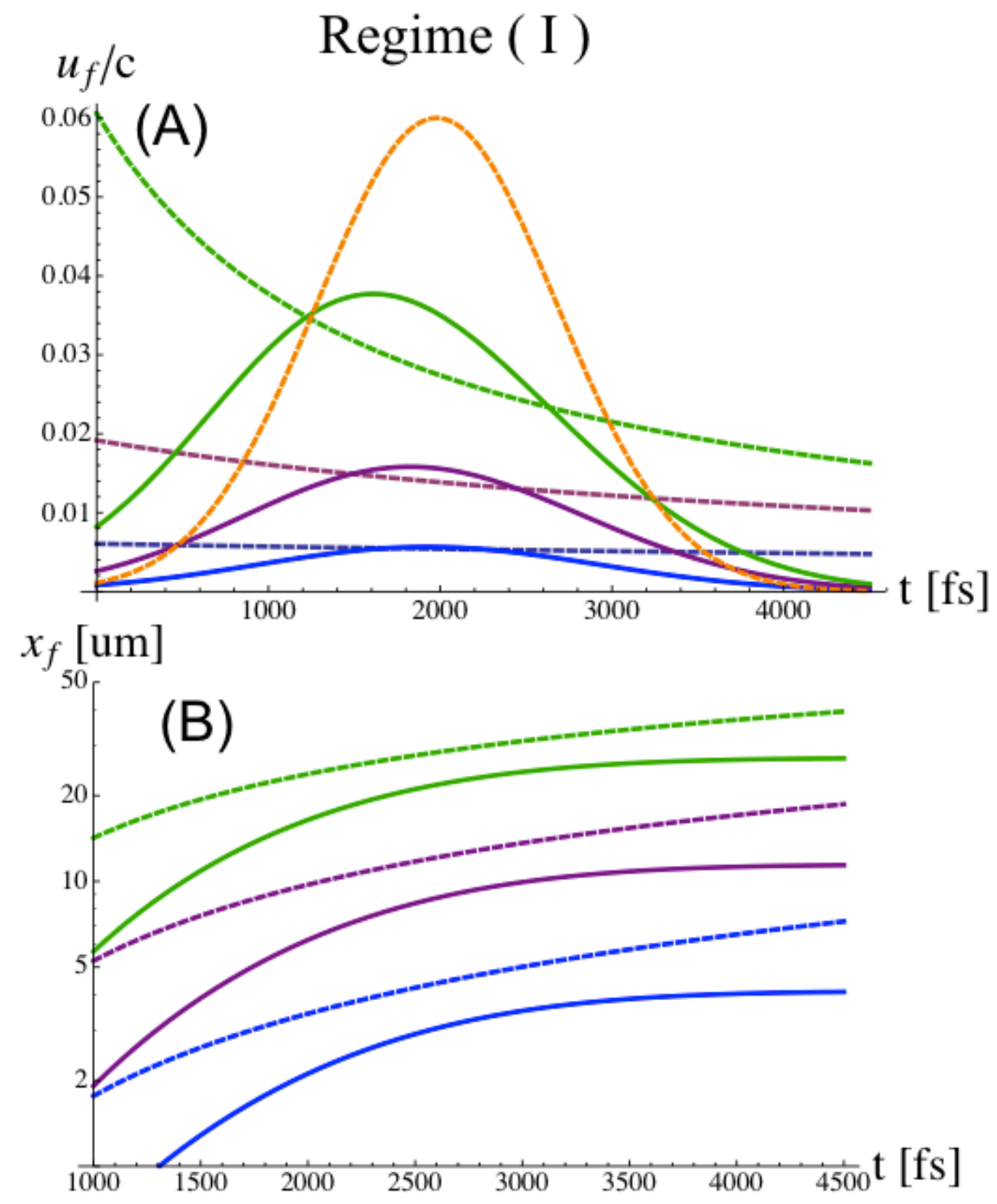

Figure 4.2 : Front velocity and hole boring depth dependence on laser intensity and pulse temporal profiles in the $\Gamma(t) \gg 1$ regime. Intensities depicted are $I_{19}$ (blue), $I_{20}$ (purple) and $I_{21}$ (green), with dashed and solid lines representing semi-infinite and Gaussian pulse envelopes respectively. (A) Front velocity $u_{f} / c$; the dashed orange line depicts the temporal pulse profile. (B) Front location $x_{f}$. System parameters are $\ell=15 \mu m, x_{s}=30 \mu m, t_{1 / 2}=1.4 p s$. 
the ion front is at rest, i.e. $\Gamma$ is unity, we see that the electron effective temperature in the interface is associated with the laser ponderomotive 'jitter.' This relation may be expressed via the rearrangement of the $u_{f}=0$ solution to Eq. (5.4):

$$
T_{e}\left(t_{s}\right)=\frac{I\left(t_{s}\right)}{c}\left[n_{0}\left(x_{f}\right)+\delta n\left(x_{f}, t_{s}\right)\right]^{-1}
$$

where $u_{f}\left(t_{s}\right)=0$ and

$$
x_{f}=\int_{0}^{t_{s}}\left[u_{f}\left(t^{6}\right)+\left(T_{e}\left(t^{\prime}\right) / M_{i}\right)^{1 / 2}\right] d t^{\prime},
$$

i.e., employing expressions (4.6) and (4.7). The effective temperature given by equation (4.9) is then related to the velocity scale for plasma expansion in the quasiisothermal regime. Fig. 4.3 (A-B) illustrate schematically the dependence of the expansion velocity on this momentum flux balance.

To obtain the time-dependence, we seek to determine a form for $T_{e}(t)$ analogous to the equation of state (EOS) closures in fluid systems. A simple form is motivated by inspection of PIC simulation results, where in Fig. $4.4(\mathrm{~A})$ we find that $(d / d t) u_{f} \simeq$ const. $\equiv \Theta$ in the equipartition regime. For our simple model, this constant $\Theta$ then fully characterizes the system with no other free parameters. The plasma effective temperature $T_{e}(t)$ is determined through,

$$
\frac{d u_{f}}{d t}-\left(\frac{1}{4 M_{i} T_{e}}\right)^{1 / 2} \frac{d T_{e}}{d t}-\Theta=0
$$

where $u_{f}(\Gamma(t) \gg 1)$ is specified. We note the free parameter may also be specified as $t_{t h}$, the time where thermal effects become non-negligible, i.e. the onset of equipartition, through $\Theta=(d / d t) u_{f}\left(t_{t h}\right)$ and the requirement $u_{f}(t)$ be continuous. 

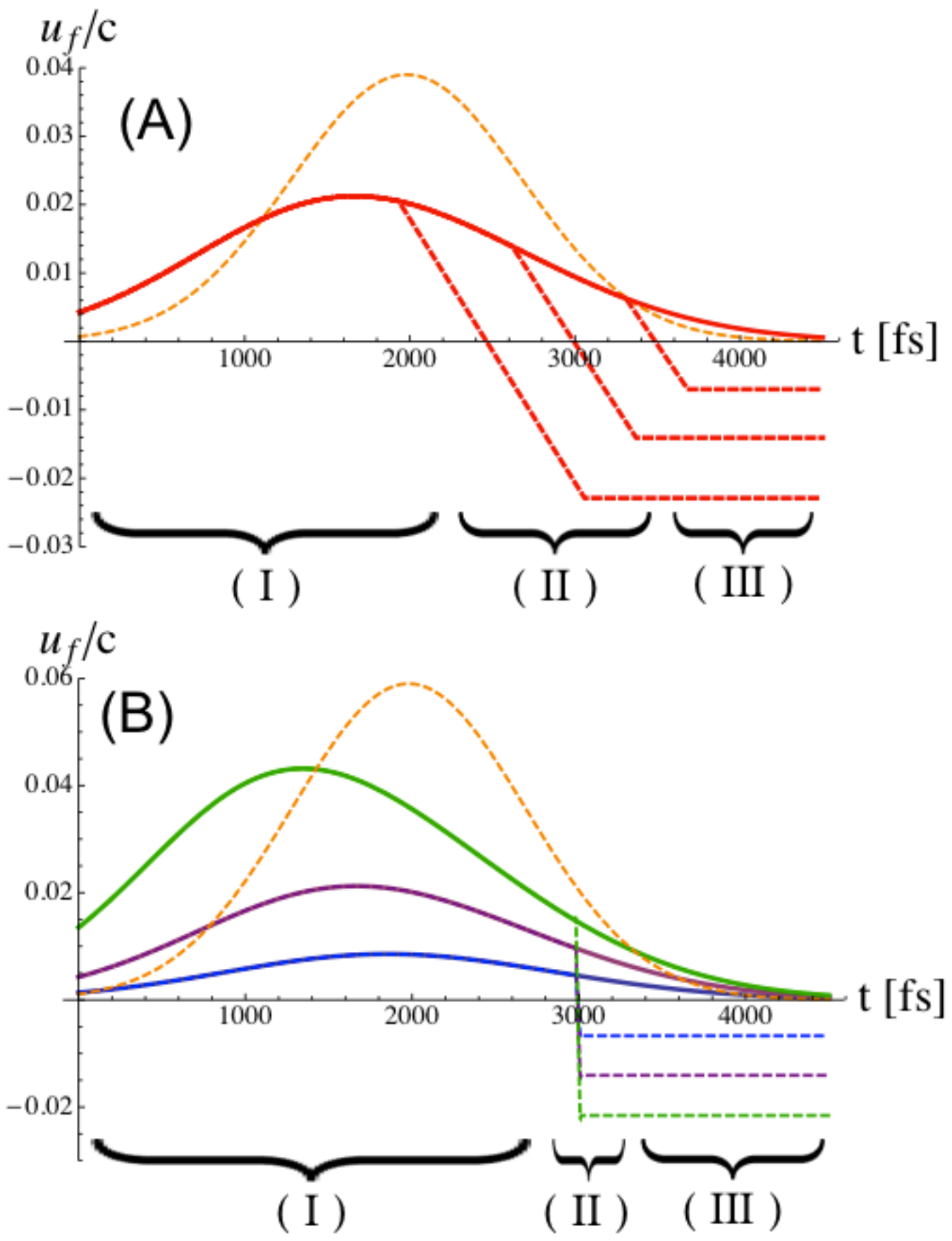

Figure 4.3 : Dependence of the characteristic expansion velocity scale $u_{t h}$ on laser intensity and $t_{s}$. (A) $u_{t h}$ for a single laser intensity $I_{20}$ for different values of $t_{s}$. (B) $u_{t h}\left(t_{s}=3 p s\right)$ for three different laser intensities, $I_{19}$ (blue), $I_{20}$ (purple), $I_{21}$ (green). The dashed orange line depicts the temporal laser pulse profile, with system parameters $\ell=10 \mu m, x_{s}=30 \mu m, t_{1 / 2}=1.4 p s$. 

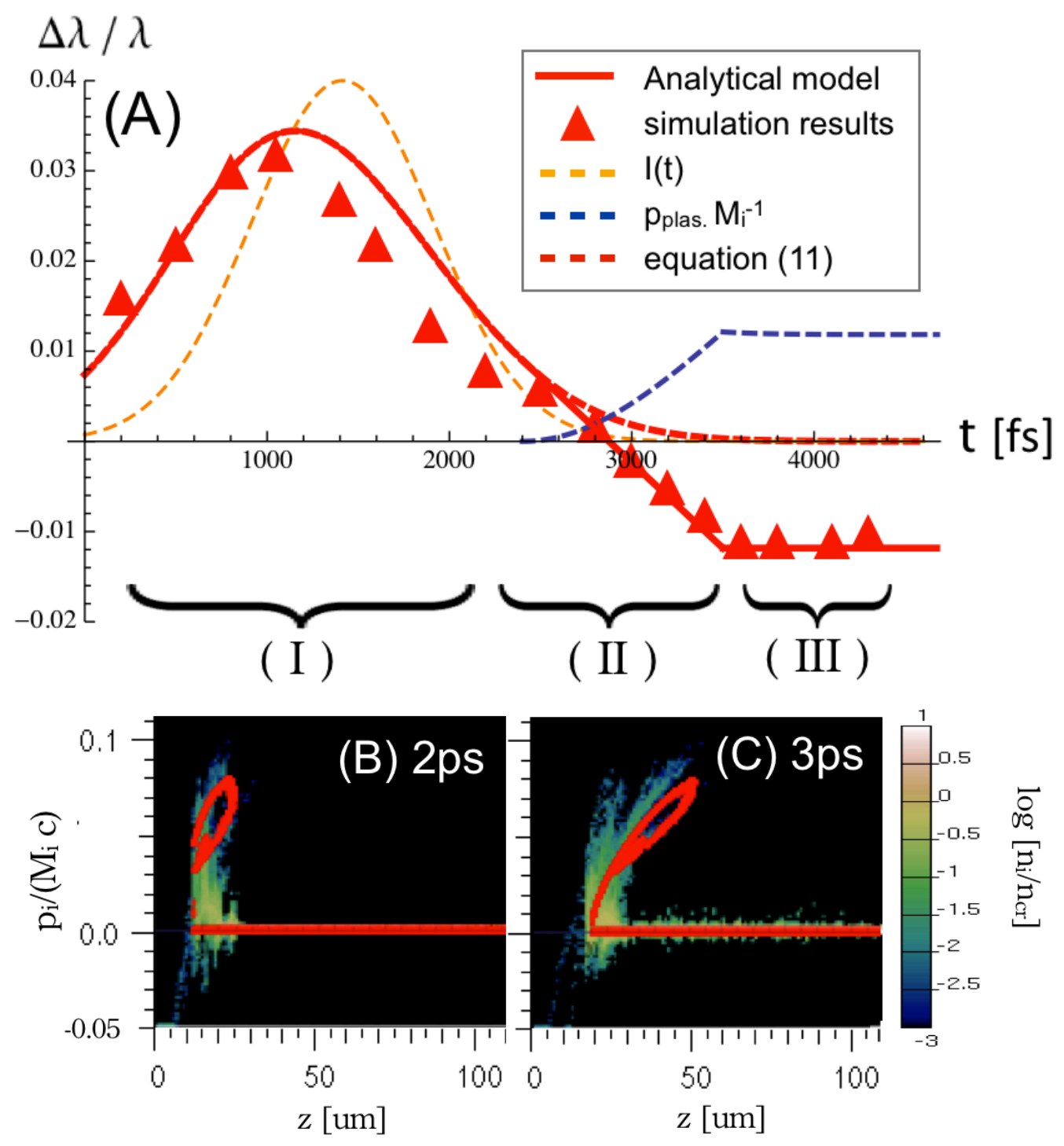

Figure 4.4 : (A) Comparison of Gaussian temporal pulse PIC simulation results with the analytical model for $\Delta \lambda / \lambda_{0}$, with regimes (I-III) labeled below. (B-C) Ion phase space density from the simulations at $2,3 p s$ in units of $\log n / n_{c r}$, with results from the Liouville code overlaid in red. 
For $\Gamma \simeq 0$, in the absence of the laser ponderomotive force, electrons from the hot plasma are free to stream out into the neighboring vacuum. This configuration sets up an ambipolar electric field which drives hydrodynamic expansion of the plasma. By equation (4.5) our simple model gives $u_{f}\left(t>t_{s}\right) \simeq-\left(T_{e} / M_{i}\right)^{1 / 2}$, i.e. reproducing the well-known solution for quasi-isothermal plasma expansion at the ion-acoustic velocity[147]. This behavior is depicted in Figs. 4.6, 4.3 and 4.4.

\subsubsection{Comparisons of the analytical model to results from PIC simula- tions}

Predictions from the simple analytical model are in good agreement with results from particle-in-cell simulations using the PSC[184] code. Figs. 4.4 and 4.5 depict the results from two simulations performed to examine the effects of a semi-infinite pulse against a Gaussian realistic pulse profile, with variable piston and plasma pressure effects self-consistently included. Both runs are one-dimensional Cartesian with a $z=150 \mu \mathrm{m}$ box and lasers of intensity $I_{0}=1.37 \times 10^{20} \mathrm{~W} / \mathrm{cm}^{2}$ at wavelength $\lambda_{0}=$ $1 \mu \mathrm{m}$. Plasma filling the box has $\ell=10 \mu \mathrm{m}$ scalelength with solid density $100 n_{c r}$ at $z_{s}=30 \mu m$, with ions of $M_{i}=2 m_{p}$ and $Z / A=1 / 2$. The numerical resolution is uniform at 16 cells/ $\mu m$, and particles are represented with 100 electrons and 50 ions per cell with particle weighting adjusted to preserve charge neutrality. Radiating and reflecting boundary conditions are used for particles and fields respectively, where particles incident upon the boundary have the component of their momenta normal to the boundary inverted. The simulation duration is $1400 \tau_{0}$ with $\tau_{0}=\lambda_{0} / c \approx 3.3$ fs. The semi-infinite pulse rises over 10 laser cycles, and the Gaussian pulse has $\alpha=1.414$ with $t_{1 / 2}=1 p s$.

Fig. 4.4 (A) depicts the simulations results for $u_{f}(t)$ for the Gaussian pulse. The 

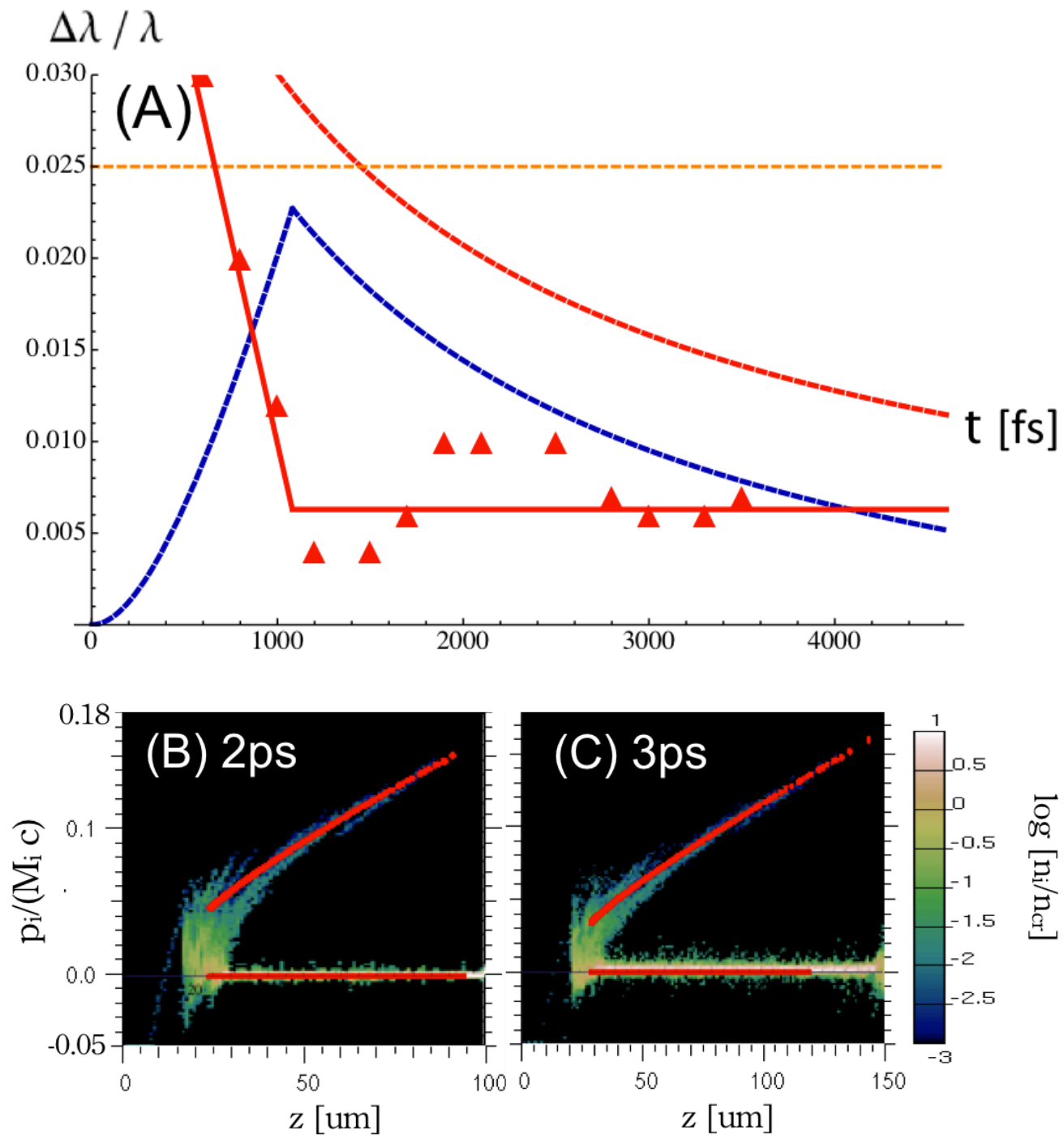

Figure 4.5 : (A) Comparison of semi-infinite pulse PIC simulation results with the analytical model for $\Delta \lambda / \lambda_{0}$, with regimes (I-III) labeled below. (B-C) Ion phase space density from the simulations at $2,3 p s$ in units of $\log n / n_{c r}$, with results from the Liouville code overlaid in red (note different transverse scales). 
change in the wavelength of light reflected from the critical surface tracks the front velocity, i.e. $\Delta \lambda(t) / \lambda_{0}=u_{f}(t)$ for $u_{f} / c \ll$. Results from the analytical model for the same parameters are depicted using $I_{0}=3 \times 10^{20} \mathrm{~W} / \mathrm{cm}^{2}$ to account for self-focusing. As the light piston accelerates into the plasma, we observe that $u_{f}(t)$ evolves through the three regimes described in Fig. 4.6. In the ponderomotive regime $\Gamma \gg 1$, the front velocity is given by equation (4.7) as the solid and dashed red lines coincide. By inspection of the data, the onset of thermal effects is taken to be $t_{t h}=2.4 p s$, and after this point $(\Gamma \simeq 1)$, the dashed blue curve shows the growth of the plasma pressure. Accordingly, the $u_{f}$ curve deviates from the ponderomotive expression through equation (4.5). In the absence of the laser driver, our simple model predicts that the plasma then expands quasi-isothermally with velocity $u_{t h} / c=-0.012$. In

plot $(\mathrm{A}), u_{f}(t)$ from the analytical model is overlaid on top of the simulation results and is shown to be in good agreement. For the semi-infinite pulse simulation, depicted in Fig. 4.5, the analytical model suggests that thermal effects become significant at early time $\sim 1.1 p s$, and the strong plasma pressure has the effect of rapidly slowing the light piston to $u_{f} / c=0.01$, far below $u_{f}(\Gamma(t) \gg 1)$. Fig. 4.4 and 4.5 (B-C) show ion phase space density plots for both temporal pulse profiles, and are observed to differ qualitatively from one another. The red overlaid curve, corresponding to ponderomotive effects, is discussed in the next section.

\subsubsection{Qualitative modifications to ion phase space}

To more closely examine the evolution of ion phase space, we have also developed a simple Liouville numerical code that evolves ions according to the ponderomotive force. The code applies the ponderomotive impulse to ions at the laser interface and evolves their trajectories through space at each timestep. The code solves $n\left(x^{6}, v^{\prime}, t^{\prime}\right)=n(x+(d / d t) x d t, v+(d / d t) v d t, t+d t)$ and the phase space density- 
conserving equation, $\left[\partial_{t}+v \cdot \partial_{x}+\left[\left(2 u_{f}(t)-u_{i}(t)\right) \delta\left(t-t\left(x=x_{f}\right)\right] \cdot \partial_{v}\right] f(x, v, t)=0\right.$, where $u_{i}$ denotes the velocities of ions at $x_{f}$ (zero for a non-accelerating piston). The code keeps track of particles accelerated beyond the laser interface and thus allows the evolution of ion phase space, including $\delta n$ ions for the accelerating light piston, to be calculated over time. Furthermore, because the Liouville code describes ponderomotive dynamics only, divergences from the PIC simulation results in the equipartition $\Gamma \simeq 1$ regime shed light on the nature of the plasma volumetric heating. Results from the code are in good agreement with the analytical results in equations (4.5-4.7) for $\Gamma(t) \gg 1$ as expected.

Fig. 4.4 and 4.5 (B-C) depict ion phase space plots from the Liouville code overlaid in red onto the PIC phase space density plots. For the Gaussian pulse, ions accelerated through $x>x_{f}$ in the PIC simulations are in approximate qualitative agreement with the Liouville ponderomotive code results. The 'loop' structure of the accelerated ions is a signature of particles that receive the ponderomotive impulse from the accelerating piston multiple times. These ions have a more complex structure, that may play an important role in collisionless shock formation[71], but are not accelerated as effectively as in the semi-infinite pulse. Extending $\sim 5 \mu m$ through critical surface, the phase space plots show evidence for non-ponderomotive ion heating. For $p_{i}<0$ particles, the high density of $p_{i} /\left(M_{i} c\right) \simeq-0.015$ ion is approximately consistent with the thermal heating at $3 p s$ from (A). For the semi-infinite pulse in Fig. 4.5 (B-C), the $x>x_{f}$ accelerated ions from simulations are in good agreement with the Liouville code. The relatively low density $n / n_{c r} \simeq 0.01$ of ions in the range $0.06<p_{i} /\left(M_{i} c\right)<0.1$ is consistent with the rapid deceleration of the piston to $1 \%$ $c$ due to the plasma pressure. The thermal effects suggested by backward-moving particles have $p_{i} /\left(M_{i} c\right) \simeq-0.02$ at the highest density, approximately consistent with the curves in plot $(\mathrm{A})$. 
In this section, a simple, more realistic model for laser hole boring in ultraintense LPI has been outlined. Studies of collisionless shocks in astrophysical settings will benefit from the investigation of the coupling of laser energy/momentum and the driving of bulk plasma flows and associated heating: jets near black holes and in gamma-ray bursts may in fact be propelled by Poynting flux-dominated outflows. A more detailed understanding of the laser light pressure interaction, demonstrated to be a time-varying driver of highly energetic ions, may thus play a key role in allowing astrophysical shock phenomena to be studied in the laboratory. Particle-incell simulations using the code PSC have been performed to test key predictions from the model, and are shown to be in good agreement. It is further shown that ions may be swept up and accelerated by the time-varying light piston multiple times, leading to more complex structures in phase space, but ultimately less effective ponderomotive acceleration of ions into the bulk target. This conclusion has obvious implications for approaches to fast ignition that propose to use light piston-driven ions to ignite the precompressed nuclear fuel. The formalism may be readily extended to two- and three-dimensions by incorporating the transverse intensity profiles of the laser beam. The multidimensional ion front quantities $\overrightarrow{x_{f}}(\vec{x}, t)$ and $\overrightarrow{u_{f}}(\vec{x}, t)$ then define the radius of curvature and the spatio-temporal evolution of the hole bored into the overdense plasma by the laser. In addition to the astrophysical applications, these profiles in turn may enable basic LPI applications such as a numerical approach to calculating hot electron distribution functions for contexts where the dominant contribution to angular divergence is related to the driving laser electric field. Such an approach was described in section 3.3, and additional basic LPI uses for diagnosing the laser and plasma conditions are discussed in the following section. 


\subsection{Predictive model for ultraintense laser preplasma using ponderomotive-driven ions}

Pre-plasma plays a crucial role in ultraintense laser plasma interactions, ranging from the enhancement of supra-ponderomotive electron generation, to the efficacy of ion acceleration mechanisms, e.g. Radiation Pressure Acceleration, to the establishment a fundamental limit for the viability of the fast ignitor approach to inertial confinement fusion. Yet, despite its integral role, there currently exist few experimental methods to diagnose pre-plasma conditions. In this letter, we propose two novel methods to diagnose laser pre-plasma through the coupling of established experimental techniques to a simple analytic model related to the ponderomotive acceleration of target-front ions. Through analytic considerations, we show that the in situ density profile can be determined with resolution to $\sim 1 \mu \mathrm{m}$ in initial plasma scalelength. In doing so, our results re-affirm the canonical ponderomotive model describing the momentum exchange between the laser and plasma. For high-contrast laser scenarios, we describe how this formalism may be employed to determine aspects of the laser temporal profile; another challenging metric to diagnose experimentally. The techniques described here may be of broad utility and specific applications are highlighted.

In this section, we propose two novel methods to diagnose laser pre-plasma through the coupling of established experimental techniques to a simple analytic model. We show that the in situ density profile can be determined with resolution to $\sim 1 \mu \mathrm{m}$ in initial plasma scalelength. For high-contrast laser scenarios, i.e. in the limit of no pre-plasma, we describe how this formalism may be employed to determine aspects of the laser temporal profile; another important yet challenging metric to diagnose experimentally. These methods may be broadly useful, e.g. demonstrating shotto-shot variations and robustness of laser energy coupling into energetic electrons 
in experimental FI contexts. Furthermore, our results underscore the connection between obtaining a monoenergetic ion energy distribution from the 'hole boring' (HB) process and the laser temporal shape.

Both methods employ a physics basis related to the ponderomotive acceleration of target-front ions by the laser. In ultraintense laser plasma interactions $\left(I>10^{18}\right.$ $\mathrm{W} / \mathrm{cm}^{2}, \tau \sim 10^{-12} s$ ), the nonlinear force related to field energy density gradients and relativistic electron currents plays a central role. At densities exceeding the relativistic critical threshold, $n /\left(\gamma n_{c r}\right)>1$ where $n_{c r}=m_{e} \omega_{L}^{2} /\left(4 \pi e^{2}\right), \gamma=\left(1+a_{0}^{2}\right)^{1 / 2}$ is related to the relativistic boosting of the electron mass and $a_{0}=e E_{L} /\left(m_{e} \omega_{L} c\right)$ is the dimensionless laser parameter, the dynamics are described by the well-established HB mechanism[215]. In a time-averaged sense, the structure near the laser-plasma interface is comprised of electrons swept out by the laser fields resulting in regions of charge compression and depletion. Ions are pulled along through the generation of an ambipolar force field, with the entire piston propagating into the bulk target. Labeling the associated velocity $u_{p}$ to denote the ponderomotive nature,

$$
\frac{u_{p}}{c}=\left(\frac{1+\eta}{2} \frac{I}{M_{i} n_{i} c^{3}}\right)^{1 / 2}=\left(\frac{Z n_{c r} m_{e} a_{0}^{2}}{2 n_{e} M_{i}}\right)^{1 / 2}
$$

where $\eta=1$, corresponding to the fraction of reflected light, has been taken for simplicity in the right-most expression. Ahead of the critical surface, due to the preplasma effect, there is often a cm- or mm-scale underdense plasma, which here we will describe through an exponential profile for simplicity, $n_{0}(x)=n_{0} \exp \left[\ell_{g}^{-1}\left(x-x_{s}\right)\right]$, with scalelength $\ell_{g}=n(d n / d x)^{-1}$ and relativistic critical surface given by $n\left(x_{c r}\right)\left(\gamma n_{c r}\right)^{-1}=$ 1. Relevant to this environment, Li et al.[120] found that in underdense, but close to critical density plasmas, the laser propagates at a velocity much lower than the linear group velocity, $u_{g} / c=\left[1-n_{0} /\left(\gamma n_{c r}\right)\right]^{1 / 2}$. Indeed, it was determined that the 
propagation velocity reached asymptotic form at the HB rate. Through equation (5.6) it is evident this rate is proportional to the density of the plasma and the irradiance of the laser. It is thus intuitive that we may exploit measurements related to the ions 'pushed' by the ponderomotive force to extract information about the properties of the plasma density and of the laser temporal envelope itself.

The first diagnostic utilizes measurements of the doppler-shifting of light reflected from ions in the critical surface over time. This concept is not new: Schumacher et al.[193] studied the properties of the reflected light beam and indeed demonstrated through particle-in-cell (PIC) simulations that light reflected from the critical surface makes an ideal diagnostic of pre-plasma. This point was also suggested by Ping et al. [162], whose work established the experimental technique measuring the doppler wavelength shift of the $2 \omega$ harmonic, $\Delta \lambda / \lambda_{0}$ of the reflected light. However, both works did not have a predictive capability, as the simulation data and the experimental data could not be matched to a self-consistent analytical model.

The primary contribution in this section is to establish this key $\Delta \lambda / \lambda_{0}$ analytic expression, taking into account both a heterogenous plasma profile and a realistic laser temporal pulse envelope for the first time. In this way, we extend the results derived by Naumova et al.[150] for the HB velocity in a heterogenous plasma (here in the $u_{p} / c \ll 1$ regime; a non-semi-infinite pulse profile significantly complicates relativistic surface motion calculations). In addition, our results provide key evidence re-affirming the canonical ponderomotive model. That is, we offer an explanation for the experimental data that is consistent with equation (5.6), without requiring a new momentum conservation hypothesis as was recently put forward in Ref. [162]. Using our model applied to the published experimental data, we demonstrate the accurate prediction of the initial plasma scalelength to within $\sim 1 \mu \mathrm{m}$.

The second diagnostic employs the same analytic model for the HB velocity, tak- 
ing into account the realistic plasma density and laser profiles, to reconstruct the target-rear ion energy distribution. Because $\mathcal{E}_{i} \propto u_{p}^{2}$, we show the pre-plasma density profile may be recovered from the time-integrated $d N_{i} / d \mathcal{E}_{i}$ distribution under certain conditions, presuming the ions accelerated by the light piston have their ballistic trajectories preserved, i.e. the target-rear sheath field from TNSA[216] is damped. The experimental technique measuring the ion energy distribution has been wellestablished, and three established experimental techniques to mitigate TNSA effects are discussed below. In carrying through these calculations, it is emphasized that obtaining a quasi-monoenergetic ion distribution through the HB mechanism is directly linked to the degree to which the laser temporal profile approximates a semi-infinite pulse.

Lastly, we briefly discuss how the model may be employed to determine aspects of the laser temporal profile in high-contrast laser scenarios, i.e. in the limit of no preplasma . To this point, the form of $I(t)$ has represented another challenging metric to diagnose in detail experimentally.

\subsubsection{Diagnosing preplasma conditions using the reflected laser light}

The simple model enabling both pre-plasma diagnostics is a closed-form, analytic expression for $\Delta \lambda / \lambda_{0}$, taking into account a heterogenous plasma profile and a realistic laser temporal pulse shape. In the $u_{p} / c \ll 1$ regime, the wavelength shift is effectively a measure of the of the HB front velocity, as a straight-foward calculation shows $\Delta \lambda / \lambda_{0}=u_{p} / c$. In deriving $u_{p}(t)$, we first note that the ion plasma density is given by $n_{i}(x, t)=n_{0}(x)+\delta n(x, t)$. The former term represents the initial plasma density and latter term describes the dynamics related to ions accelerated by the light piston at earlier time. By expressing the laser (HB) front as $x_{f}(t)=\int_{0}^{t} u_{f}\left(t^{6}\right) d t^{\prime}$, equation (5.6) may be reformulated in terms of the total instantaneous plasma density at the 
piston, $n(t)=n_{0}\left(x_{f}\right)+\delta n\left(x_{f}, t\right)$. For most common scenarios, because $\delta n / n_{0} \ll 1$ the latter term does not strongly affect the piston motion and will be neglected in this work. However, for rapidly time-varying pulses, we note that ions may be swept up and accelerated by the light piston multiple times, leading to a novel richness in phase space. For an exponential density pre-plasma of the form described above, let us first calculate the analytic HB velocity for a semi-infinite laser pulse of intensity $I_{0}$. Employing separation of variables, we may solve for the front motion,

$$
u_{p}(t)=u_{p 0} e^{x_{s} /\left(2 \ell_{g}\right)}\left[1+e^{x_{s} /\left(2 \ell_{g}\right)} \frac{u_{p 0}}{2 \ell_{g}} t\right]^{-1}
$$

This expression is formulated in terms of the characteristic ponderomotive velocity for the system, $u_{p 0} \equiv\left[I_{0} /\left(M_{i} n_{0} c\right)\right]^{1 / 2}=\left[Z m_{e} n_{c r} a_{0}^{2} c^{2} /\left(2 M_{i} n_{p e 0}\right)\right]^{1 / 2}$. This is the (constant) velocity associated with the peak laser intensity pushing on the peak plasma density in the system.

Now let us consider a realistic temporal pulse profile, here a Gaussian of the form $I(t)=I_{0}(t) \exp \left[-2\left(t-\alpha t_{1 / 2}\right)^{2} / t_{1 / 2}^{2}\right]$ where $\alpha$ determines $I\left(t_{0}\right)$. The coefficient $\partial_{t} I_{0}(t) \neq 0$ may be employed to describe nonlinear effects, e.g. laser self-focusing[46] for $P_{L} / P_{c}=P_{L}\left(17 G W n_{c r} / n_{p e 0}\right)^{-1}>1$. Assuming a constant $I_{0}$, a closed-form solution may be obtained,

$$
\begin{gathered}
u_{p}(t)=u_{p 0} e^{x_{s} /\left(2 \ell_{g}\right)} e^{-\left(t-\alpha t_{1 / 2}\right)^{2} t_{1 / 2}^{-2}} \times \\
{\left[1+e^{x_{s} /\left(2 \ell_{g}\right)} \frac{\sqrt{\pi} u_{p 0} t_{1 / 2}}{4 \ell_{g}} \Omega(t)\right]^{-1}}
\end{gathered}
$$

where $\Omega(t) \equiv \operatorname{erf}(\alpha)+\operatorname{erf}\left[\left(t-\alpha t_{1 / 2}\right) / t_{1 / 2}\right]$ and the error function is employed.

It is clear that equations (4.13) and (4.14) describe qualitatively distinct curves for $u_{p}$, and thus of the energy spectra associated with the ponderomotively-driven 


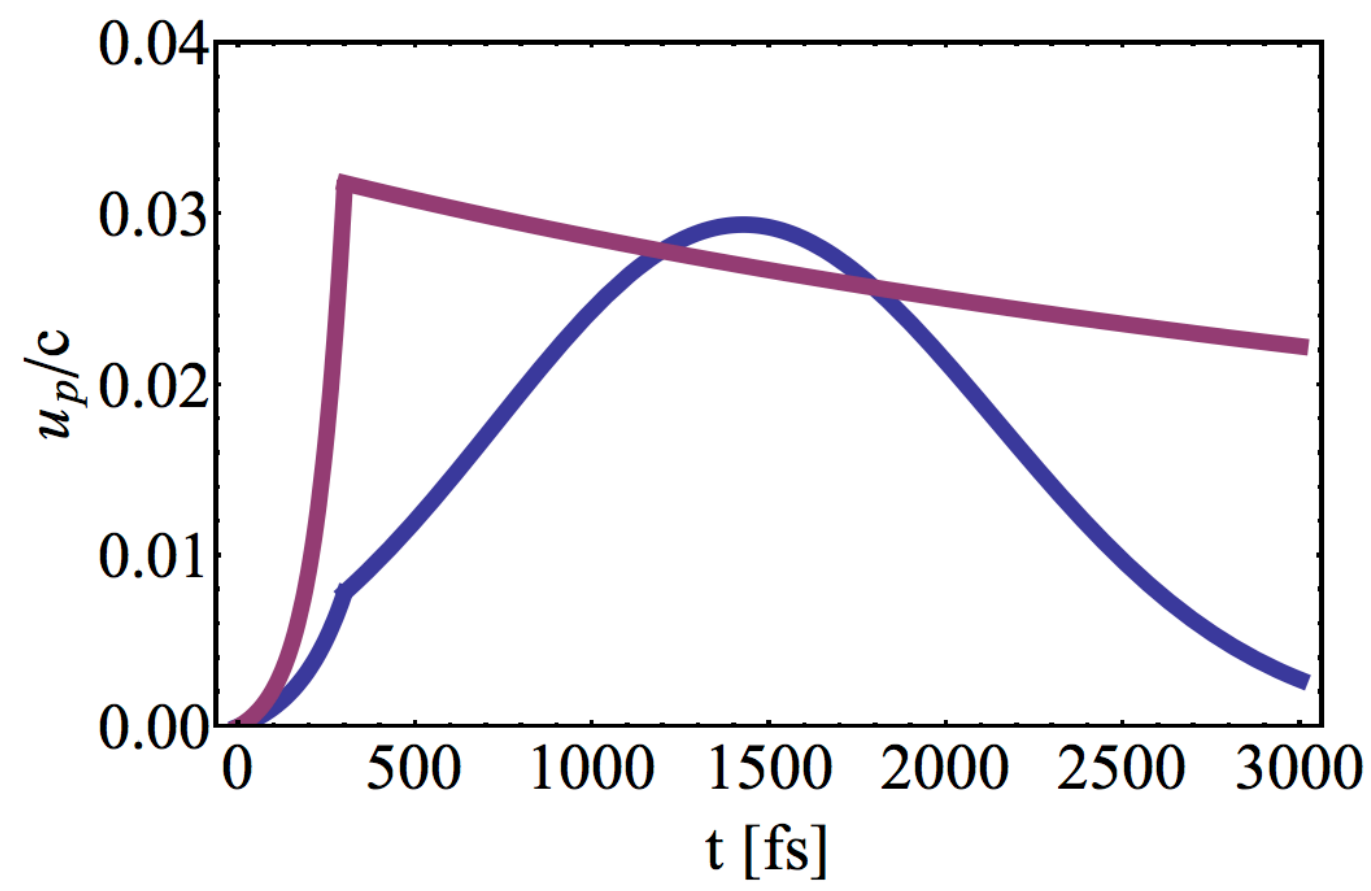

Figure 4.6 : Schematic depicting the analytic front velocity $u_{p}$ curves for the semiinfinite (purple) and Gaussian temporal (blue) pulse shapes, through equation (4.13) and (4.14) respectively. For both shapes, the peak laser intensity is $4 \times 10^{20} \mathrm{~W} / \mathrm{cm}^{2}$ and the plasma conditions are $\ell_{g}=30 \mu \mathrm{m}, x_{s}=100 \mu \mathrm{m}, n_{0} / n_{c r}=100$ and $M_{i} / m_{p}=2$. The Gaussian pulse peaks at $1.5 p$ with a full-width half-maximum of $0.5 p s$.

ions. This is illustrated in Fig. 4.6, where each curve is depicted for the same plasma parameters and peak laser intensity. At early time prior to the arrival of the main laser pulse $(t<0$, not depicted), the plasma critical surface slowly expands with $u_{p}<0$ at a rate determined by bulk heating associated with the nanosecond-scale ASE drive. As the ultraintense laser impinges upon the target, it propagates through the underdense plasma until reaching an interface of $n_{i} /\left(\gamma n_{c r}\right) \sim 1$. $u_{p}$ then increases rapidly as momentum flux is conserved in the HB interaction between the laser and the plasma (the transition is depicted schematically in the figures as an exponential rise from zero to $300 \mathrm{fs}$ ). 


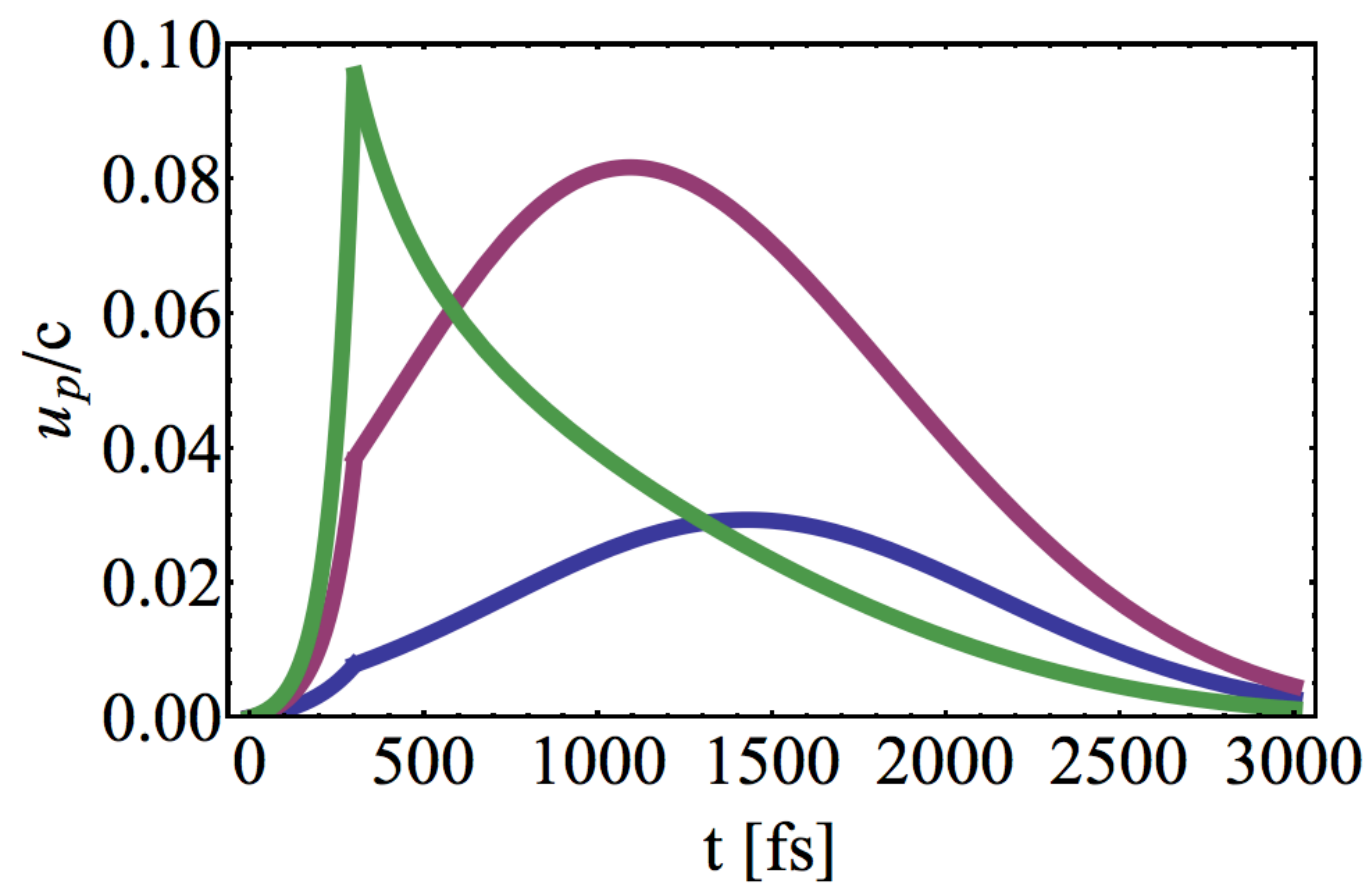

Figure 4.7 : Front velocity dependence on pre-plasma scalelength for a Gaussian temporal laser pulse. Plasma and laser conditions are the same as in Fig. 4.6, with the scalelength varying from $\ell_{g}=30 \mu \mathrm{m}$ (blue), $15 \mu \mathrm{m}$ (purple) and $3 \mu \mathrm{m}$ (green). The curve is seen to peak sharply at early time for steep density profiles.

Having established the distinction between the $\Delta \lambda / \lambda_{0}$ curves for semi-infinite and Gaussian-temporal pulses, we now consider changes in the form of the HB velocity as the pre-plasma scalelength is varied, keeping other parameters fixed. Fig. 4.7 illustrates the evolution of $u_{p}$ for Gaussian pulses as a function of the initial plasma density profile. For steep plasma density profiles, the peak in $u_{p}$ is seen to shift to earlier time, away from the peak in laser intensity.

The strong, nonlinear dependence of $u_{p}$ on $\ell_{g}$ in equation (4.14) suggests that measurements of $\Delta \lambda / \lambda_{0}$ can indeed be employed to infer pre-plasma conditions. To understand the quantitative agreement, we now apply this model to the experimen- 
tal conditions from Ref. [162]. The experimental parameters are used as input to the model, with $x_{s}=115 \mu m, \alpha=1.414, t_{1 / 2}=1.4 p s$ and the nominal peak intensity $I_{0}=8 \times 10^{19} \mathrm{~W} / \mathrm{cm}^{2}$. Fig. 4.8 depicts the analytic $u_{p}$ curves from equation (4.14) with these parameters, for a variety of pre-plasma scalelengths, as well as the experimental measurements of $\Delta \lambda / \lambda_{0}$. The results of our simple analytical model are seen to be in very good agreement with the data through $2 \mathrm{ps}$, predicting $\ell_{g} \simeq 3.5 \mu \mathrm{m}$, close to the $3 \mu \mathrm{m}$ scalelength inferred in Ping et al. The good agreement between the $3.5 \mu \mathrm{m}$ analytical prediction and the experimental data further suggests that the model captures the essential multi-dimensional physics features. (As an aside, we have also tested the model against the on-target laser intensity, quoted as $I_{\text {targ }} / I_{0} \simeq 2$; differences between this and the results presented here are negligible, as for the experimental parameters $n_{i} / n_{c r} \gg \gamma_{0}$.)

Our results indicate that the system dynamics remain ponderomotive in the canonical sense[215] until late time. That is, the HB velocity is seen to evolve according to equation (5.6) and (4.14), until late time, around 2.5ps. The $t>2.5 p s$ regime may be addressed in future work. This result stands in contrast to the new theoretical model proposed in Ping et al. suggesting that momentum exchange between the laser and plasma becomes non-ponderomotive for irradiances of the order $\sim 10^{21} \mathrm{~W} / \mathrm{cm}^{2}$ around 2ps, the time of the peak laser pulse intensity. Nevertheless, the utility of the experimental techniques and $\Delta \lambda / \lambda_{0}$ measurements established in this work in understanding features of the laser plasma interaction cannot be overstated.

In effect, the analytical model in equation (4.14) maps a set of input parameters $I(t), n_{0}(x)$ to a front velocity $\Delta \lambda / \lambda_{0}\left(=u_{p} / c\right)$. For certain parameter regimes, the mapping has resolution to $\sim 1 \mu \mathrm{m}$ in initial plasma scalelength. Key predictions of the model are also in good agreement with results from new PIC simulations (not 


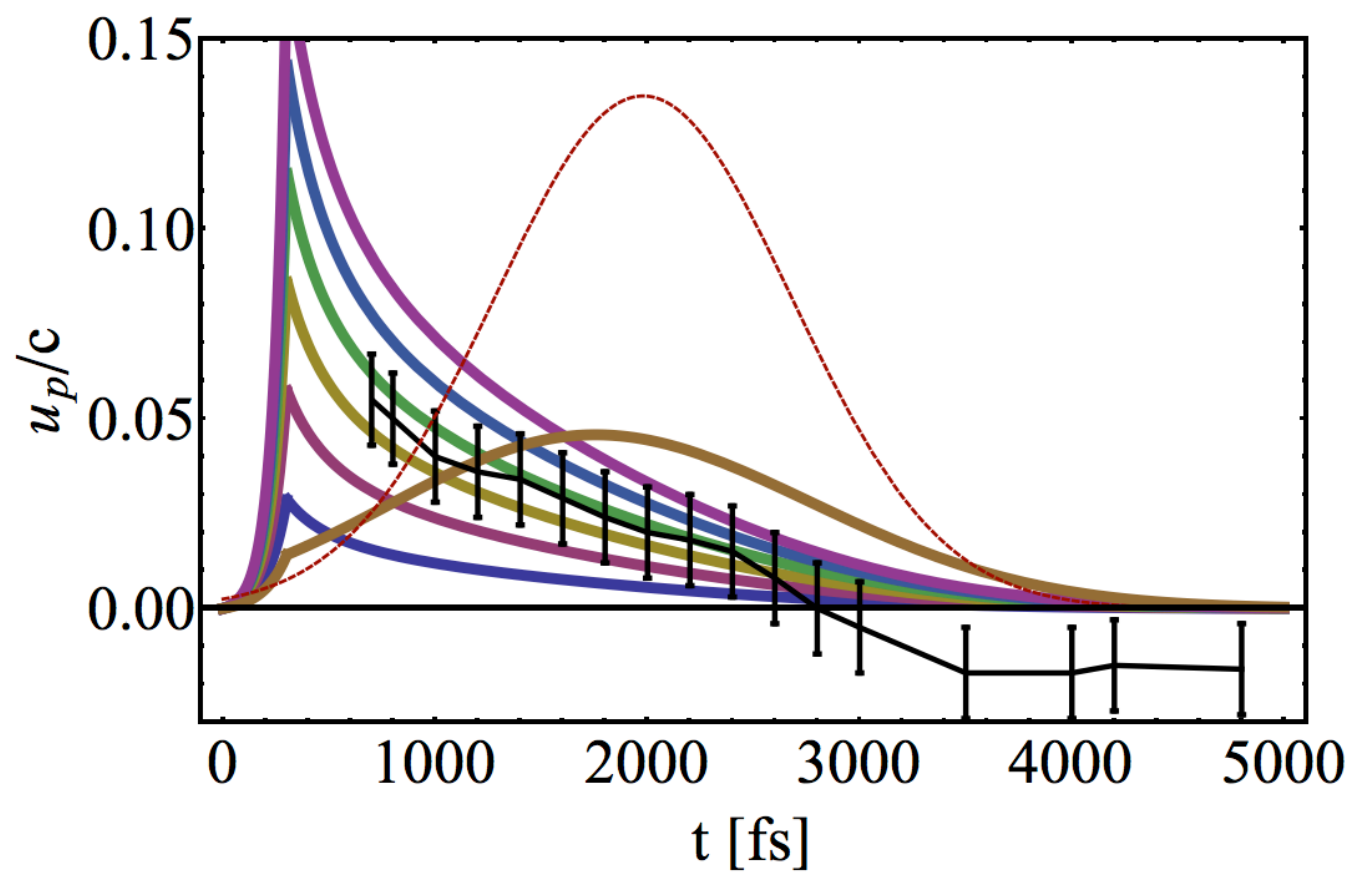

Figure 4.8: Application of the analytical model for $u_{p}$ to existing experimental data[162]. The thin dashed-red line represents the normalized laser intensity profile. The solid colored curves represent pre-plasma scalelength, varying in $1 \mu \mathrm{m}$ intervals from $\ell_{g}=1 \mu \mathrm{m}$ (innermost blue) to $6 \mu \mathrm{m}$ (outermost purple), with the outlying dark yellow curve representing $30 \mu \mathrm{m}$ for comparison. The black data points and error bars of \pm 0.012 in $\Delta \lambda / \lambda_{0}$ correspond to the experimental data (see text). 
pictured here).

\subsubsection{Diagnosing preplasma conditions using the time-integrated ion en- ergy spectrum}

Because the pondermotive-driven ions contain information about the laser intensity and plasma density profiles, the pre-plasma conditions may in principle be deconvolved from measurements of the ion energy distribution. In order to do this, the ballistic trajectories of ions accelerated by the light piston must be preserved. It then follows that the time-integrated $d N_{i} / d \mathcal{E}_{i}$ distribution will only be useful if the the target-rear sheath field from TNSA[216] is damped on a faster timescale than the ion ballistic timescale $\sim \ell_{\text {target }} / u_{p}$, where the numerator represents the target longitudinal extent. Three experimental techniques to do so have been established: (1) long-pulse irradiation of the target back side[216], (2) use of gas jet targets with

an intrinsic density gradient along the back[71], and (3) use of a circularly-polarized pulse to avoid electron overheating[150].

Presuming the TNSA field is absent, we present a simple Liouville numerical code that computes the $d N_{i} / d \mathcal{E}_{i}$ spectrum for a given set of parameters, $I(t), n_{0}(x)$. (An analytical expression is challenging for a realistic pulse profile; for a semi-infinite pulse the distribution was obtained in Naumova et al.[150]) The code applies the ponderomotive impulse to ions at the laser interface and evolves their trajectories through space at each timestep. The code solves $n\left(x^{6}, v^{\prime}, t^{\prime}\right)=n(x+(d / d t) x d t, v+$ $(d / d t) v d t, t+d t)$ and the phase space density-conserving equation,

$$
\begin{array}{r}
{\left[\partial_{t}+v \cdot \partial_{x}+\left[\left(2 u_{p}(t)-u_{i}(t)\right) \delta\left(t-t\left(x_{f}\right)\right] \cdot \partial_{v}\right]\right.} \\
f(x, v, t)=0
\end{array}
$$



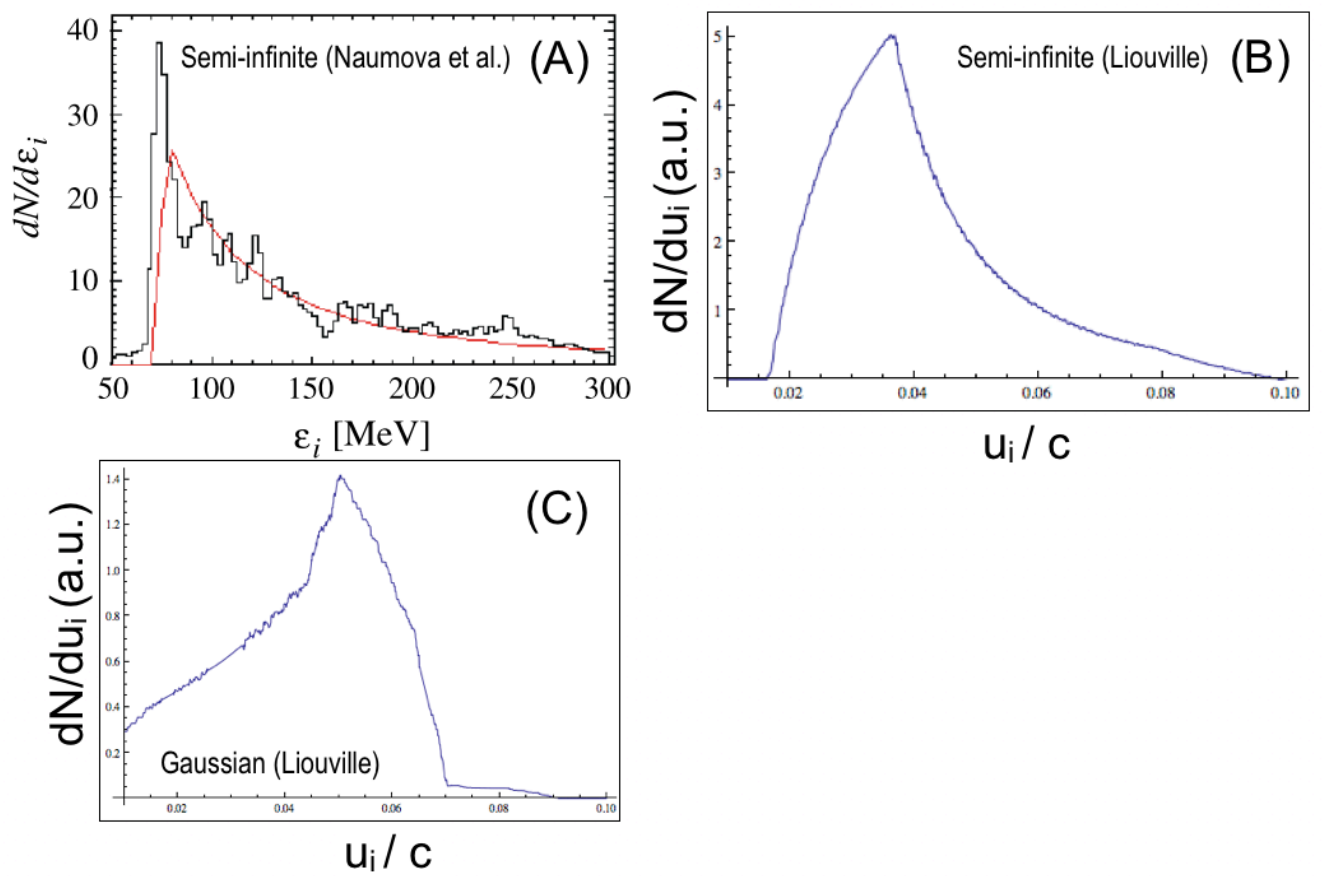

Figure 4.9 : Time-integrated target-rear ion spectra for the parameters used in Naumova et al.[150]. (A) shows the simulation results along with their analytic model (red curve) for a semi-infinite pulse envelope. (B) shows the distribution from our Liouville numerical code for the same conditions. (C) shows the Liouville results for the same parameters, using a Gaussian temporal pulse (see text). 
where $u_{i}$ denotes the velocities of ions at $x_{f}$ (zero for a non-accelerating piston). The code keeps track of particles accelerated beyond the laser interface and thus allows the evolution of ion phase space, including $\delta n$ ions for the accelerating light piston, to be calculated over time.

Through comparisons between this spectrum and the measured spectrum, the preplasma scalelength can be recovered. For reference, Fig. 4.9 (A) shows the $d N_{i} / d \mathcal{E}_{i}$ analytic spectrum from Naumova et al. for a semi-infinite laser pulse, analogous to the computation performed by our Liouville numeric code. Comparisons with the simulation results show good agreement. Plot (B) shows the results from the Liouville code for the same parameters as in (A), reproducing the functional shape and approximate distribution from both the analytic model and simulation data from Naumova et al.. (C) shows the change in the ion energy distribution for the same parameters, using a Gaussian pulse profile, from the Liouville code.

\subsubsection{Application to measuring laser parameters in high-contrast situa- tions}

For high-contrast scenarios, i.e. in the limit of no pre-plasma , or where the preplasma conditions have been established through other means, this formalism may be employed to determine the laser temporal profile. To date, this has proven to be another challenging metric to diagnose experimentally. By analogy to equation (4.14), we may derive an expression for the front velocity for a Gaussian-temporal pulse incident on a plasma of $\ell_{g} \rightarrow 0$,

$$
u_{p}(t)=u_{p 0} e^{-\left(t-\alpha t_{1 / 2}\right)^{2} t_{1 / 2}^{-2}}
$$




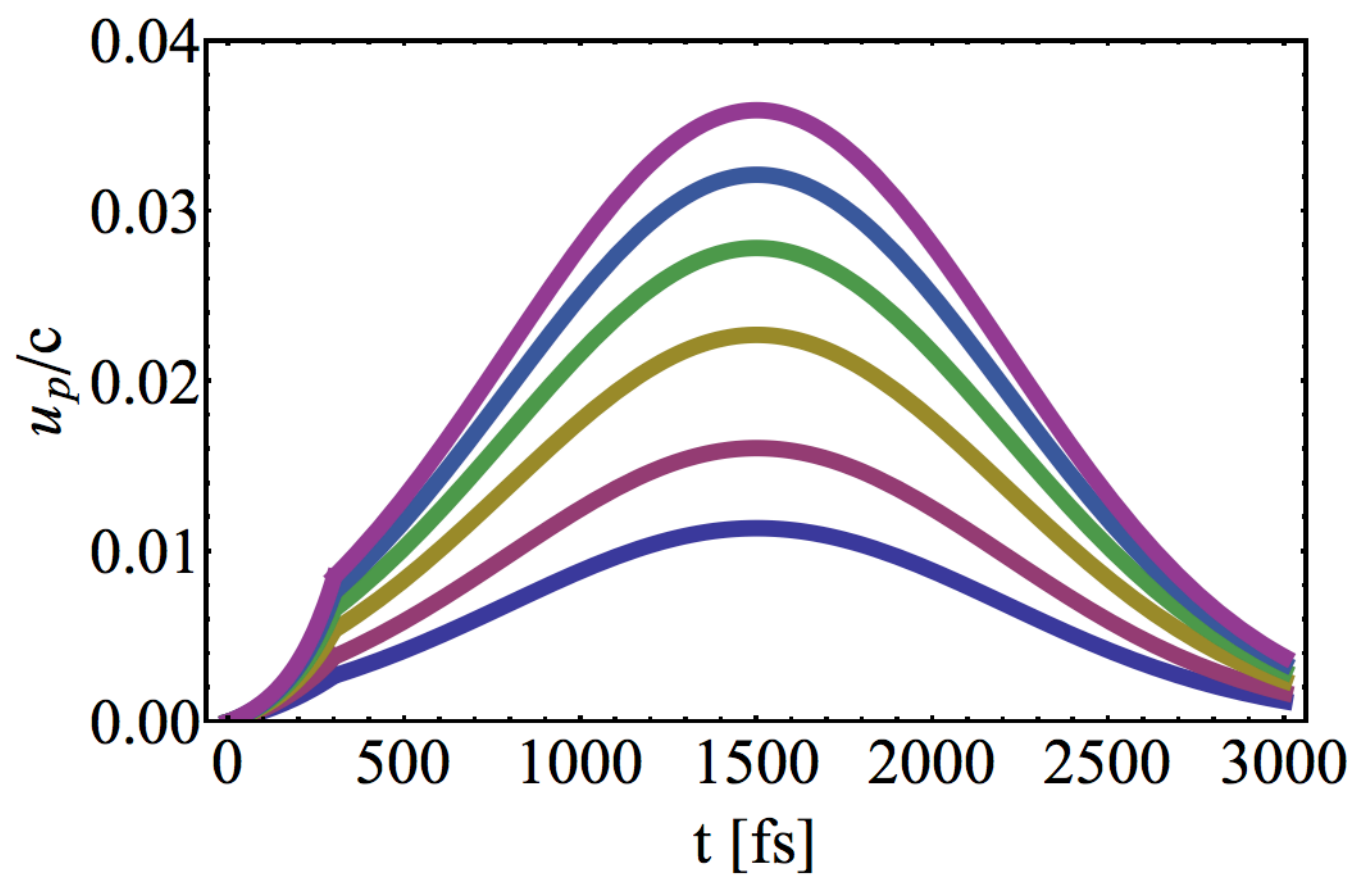

Figure 4.10 : Analytic $u_{p}$ curves from equation (4.16) in the high-contrast pulse limit. Starting from the inner-most blue curve of $I_{0}=1.3 \times 10^{20} \mathrm{~W} / \mathrm{cm}^{2}$ and moving outward, each colored curve represents an increase in laser intensity by a factor of two, such that the outer-most purple curve has $I / I_{0}=10$.

In the case where $I_{0}$ in $u_{p 0}$ is to be determined, Fig. 4.10 illustrates the sensitivity of the curve, in proportion to $I^{1 / 2}$. For these parameters, the disparity between the $u_{p}$ forms shown should allow the determination of the peak laser intensity with resolution $I / I_{0} \simeq 2$, i.e. outside of the Specular FROG error bars of \pm 0.012 in $\Delta \lambda / \lambda_{0}$ demonstrated in Ref. [162].

In summary, we have outlined two novel methods to diagnose laser pre-plasma conditions through the coupling of a simple analytic model to experimental measurements using well-established techniques. Both methods employ a physics basis related to the ponderomotive acceleration of target-front ions by the laser, and we show that the in situ density profile can be determined with resolution to $\sim 1 \mu \mathrm{m}$ 
in initial plasma scalelength. The model effectively maps a set of input parameters $I(t), n_{0}(x)$ to a front velocity $\Delta \lambda / \lambda_{0}\left(=u_{p} / c\right)$. For high-contrast laser scenarios we have described how this formalism may be employed to determine aspects of the laser temporal profile.

The first method utilizes measurements of the doppler-shifting of light reflected from ions in the critical surface over time. The second method works by reconstructing the target-rear time-integrated $d N_{i} / d \mathcal{E}_{i}$ ion energy distribution. The latter method offers a lower resolution determination susceptible to degeneracy under certain conditions, but may be convenient in experiment as a 'ride-along' diagnostic to obtain an estimate of the initial density profile. The Liouville numerical code employed by the authors to calculate the $d N_{i} / d \mathcal{E}_{i}$ profile is written in Mathematica and will be made available online for public use.

A key prediction of our results is that the system dynamics remain ponderomotive in the canonical sense[215] until late time. That is, we offer an explanation for the experimental data[162] that is consistent with equation (5.6), without requiring a new momentum conservation hypothesis as was recently suggested in Ref. [162].

These methods may be of broad utility in that they provide novel means to diagnose a parameter crucial to the outcome of ultraintense laser experiments, on a shot-to-shot basis. In addition, we note that it is straight-forward to extend this formalism to two- and three-dimensions by incorporating the transverse intensity profiles of the laser beam. The multidimensional $\overrightarrow{x_{p}}(\vec{x}, t), \overrightarrow{u_{p}}(\vec{x}, t)$ then define the radius of curvature and the spatio-temporal evolution of the hole bored into the overdense plasma by the laser. These profiles in turn may enable a variety of basic LPI applications, such as a numerical approach to calculating hot electron distribution functions for contexts where the dominant contribution to angular divergence is related to the driving laser electric field. As illustrated in Fig. 4.7, the model described here opens 
to the door to a richer space of ponderomotive time history curves, and thus also to a richer space of ion energy distributions. As such, it may be further useful in the context of accelerator applications, enabling the tailoring and shaping of energetic ion beams generated through the hole boring process. 


\section{Chapter 5}

\section{Focusing of intense subpicosecond laser pulses in wedge targets}

In this section two dimensional particle-in-cell simulations characterizing the interaction of ultraintense short pulse lasers in the range $10^{18} \leq I \leq 10^{20} \mathrm{~W} / \mathrm{cm}^{2}$ with converging target geometries are presented. Seeking to examine intensity amplification in high-power laser systems, where focal spots are typically non-diffraction limited, we describe key dynamical features as the injected laser intensity and convergence angle of the target are systematically varied. We find that laser pulses are focused down to a wavelength with the peak intensity amplified by an order of magnitude beyond its vacuum value, and develop a simple model for how the peak location moves back towards the injection plane over time. This performance is sustained over hundreds of femtoseconds and scales to laser intensities beyond $10^{20} \mathrm{~W} / \mathrm{cm}^{2}$ at $1 \mu \mathrm{m}$ wavelength. Thus, this scheme could enable focusing high power laser light to higher intensities, in a fashion that could support reaching the QED-plasma regime[170].

To distill and examine key physical processes in this situation we use PIC simulations to model wedge targets in the limit of no prepulse, i.e. high-contrast laser pulse propagation in a cleared channel. The effects of prepulse in a closed cone, for a single angle, were reported on in MacPhee et al.[134] Recent numerical work published by other groups has indicated that peak laser focusing of $\sim 15$ times could be achieved in a small spot size of radius $\sim 1 \lambda_{0}$, using hollow tip cone (wedge) targets.[222] It was proposed that the intense, highly-focused beams generated by these configurations 
could have applications for HEDP fields such as $K_{\alpha}$ based backlighting. However, these simulations were performed in the limit of an optical physics model with static boundaries[222], and further research has considered evolving weakly overdense target walls with lasers of intensity $I<10^{20} \mathrm{~W} / \mathrm{cm}^{2}$ over $\sim 350$ fs [220]. The results presented in this section take into account the additional effects of hot electron generation and phase space evolution, and describe nonlinear laser-plasma interaction in $100 \times n_{c r}$ targets over picosecond timescales. We consider a pulse-target parameter space that represents a sampling of recent literature[86, 134, 222], and is comprised by $1 \mu \mathrm{m}$ wavelength lasers with intensities in the range $10^{18}-10^{20} \mathrm{~W} / \mathrm{cm}^{2}$ and targets with half-angles of $17^{\circ}, 30^{\circ}$ and $45^{\circ}$. We identify key dynamical features and trends as laser intensity and target angle are systematically varied, such as the generation of a dominant hot electron filament aligned with the target tip for $a>1$ pulses in the $17^{\circ}$ target. In these configurations particle acceleration mechanisms in the underdense plasma, most notably self-modulated laser wakefield acceleration[46, 166] (SM LWFA), excite electrons to several times the ponderomotive potential. By contrast the wide $45^{\circ}$ target exhibits two equally dominant electron filaments off to the sides of the tip, with electron acceleration through the tip effectively suppressed. We interpret this trend in the context of the relative efficiencies of ponderomotive selfchanneling[166] and collisionless laser energy absorption mechanisms such as Brunel heating[24] near the critical interface. For pulses with intensity higher than $I_{19}$ in the $45^{\circ}$ target, over time the laser pressure becomes insufficient to clear plasma ablated from the target walls from the laser channel. This results in effective closure of the target tip.

The $17^{\circ}-I_{20}$ pulse-target configuration is identified as optimal with peak laser focusing $I_{\text {peak }} / I_{0} \approx 8.8$ and maximal electron flux density $P_{e} / I_{0} \approx 3.2$ at the target tip. This $I_{\text {peak }}$ represents an $82 \%$ focusing efficiency compared to an ideal optical 
simulation with static boundaries, with losses primarily going into electron heating. Also observed in this geometry is a clear trend where the region of peak laser intensity regresses away from the target tip at intensity-dependent rates that saturate at $I \gg 10^{19} \mathrm{~W} / \mathrm{cm}^{2}$. Interestingly, this trend suggests the $17^{\circ}$ geometry may be representative of a broader class of pulse-target configurations that are characterized by a sustained dynamical equilibrium between plasma expansion and laser radiation pressure. A general analytical model is developed for these systems that predicts the front velocity $u_{f}$ and yields key scalings $I_{\text {peak }}, P_{e} \propto a^{2}$, in good agreement with the $17^{\circ}$ target results. This unique class of wedge target configurations has highly desirable properties and may have applications for the design of $K_{\alpha}$ based X-ray backlighters to image ICF implosions and other HEDP experiments.[157]. In this context they represent experimentally simple systems to achieve bursts of $10 \mathrm{x}$ boosted light for hundreds of femtoseconds, focused down to small spots of $\sim 1 \lambda_{0}$. As X-ray image resolution is proportional to source size, this small spot may enable high resolution radiography, while the amplified laser intensity drives a higher energy flux over a fixed time interval for the snapshot. Because the $I_{\text {peak }}$ and $P_{e}$ scalings extend to the regime $I \gg I_{20}$, these configurations may become even more attractive as higher intensity short pulse laser systems such as NIF-ARC[90] come online in the future.

\subsubsection{Numerical modeling of laser focusing in wedge targets}

We use the particle-in-cell code PSC[184], which employs the Finite-Difference-TimeDomain (FDTD) numerical scheme to move particles according to the Lorentz force equation and evolve fields according to Maxwell's equations on a discrete grid. We present the results of nine simulations, representing all combinations of pulse intensities $I_{0}=1.37 \times\left\{10^{18}, 10^{19}, 10^{20}\right\} W / \mathrm{cm}^{2}$ corresponding to $a=\{1,3.16,10\}$ at wavelength $\lambda_{0}=1 \mu \mathrm{m}$, and target opening half-angles of 17,30 , and $45^{\circ}$. For conve- 
nience we define the pulse-target notation $a_{n} \theta_{m}$ where $n, m$ index the dimensionless laser parameter and target-half angle in degrees. Selection of the $\theta_{17}$ target is motivated by recent optical simulations that indicate peak laser focusing could reach 15 times in $16-18^{\circ}$ hollow tip cone (wedge) targets[222]. Note that irrespective of angle the targets have a fixed height, thickness, and tip width of $30 \lambda_{0}, 4.2 \lambda_{0}$, and $\Delta y_{t i p}=5 \lambda_{0}$ respectively. Particles are represented with 120 electrons and 30 ions per cell, yielding a total of $\sim 10^{7}$ quasi-particles initially. Ions are efficiently modeled using fewer particles due to their high inertia and particle weighting is adjusted to preserve charge neutrality. Radiating and reflecting boundary conditions are used for particles and fields respectively, where particles incident upon the boundary have the component of their momenta normal to the boundary inverted. Our simulations are two dimensional Cartesian with system size $50 \lambda_{0} \times 40-84 \lambda_{0}$ in the longitudinal$\hat{z}$ and transverse $-\hat{y}$ directions respectively, comprising a mesh of $1600 \times 1280-2688$ cells with uniform grid size $\Delta y=\Delta z=0.031 \lambda_{0}$. The simulation duration is $550 \tau_{0}$, with $\tau_{0}=\lambda_{0} / c \approx 3.3$ fs and timestep $\Delta t \approx 0.045$ fs as determined by the Courant criterion[11].

Laser pulses in the simulations presented here are super-Gaussian in the transverse direction with $I(y)=I_{0} \times \exp -\left(y / y_{0}\right)^{8}$. Temporal profiles are Gaussian and rise over 10 optical cycles with a semi-infinite pulse envelope. Fig. 5.1 shows the laser propagating upward into the system from the injection plane at $z=0$, with the target tip at $z_{t i p}=35 \lambda_{0}$. The laser electric field is linearly polarized in the simulation plane. The beam half-width half-maxima $(\mathrm{HWHM})$ are $w_{0}=\{6.0,9.9,15.1\} \times \lambda_{0}$ for targets half-angles $\theta_{17}, \theta_{30}$ and $\theta_{45}$ respectively, a constant fraction of the target base width. Note that we take $w(z)=w_{0}$, as the expected beam divergence is negligible over the target longitudinal height. Consider an analogous Gaussian beam with waist $w_{0}$, 


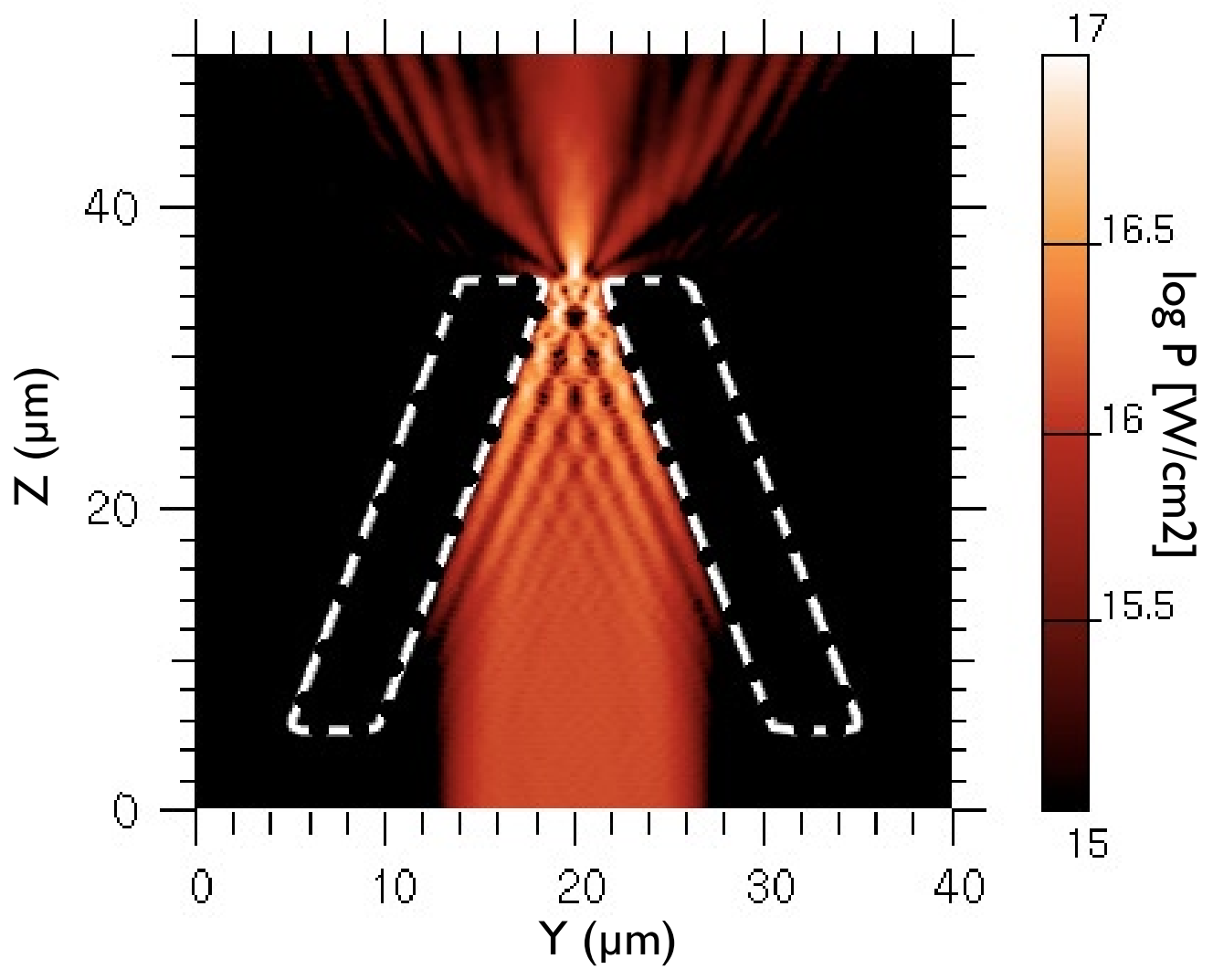

Figure 5.1 : Schematic for the $\theta_{17}$ target configuration with the low intensity $a=0.1$ reference beam (see text); laser longitudinal Poynting flux $P_{z}$ shows $I_{\text {peak }} / I_{0}=10.8$ peak focusing with superimposed white dashed lines indicating initial target position. 
where we see the vacuum diffraction length $z_{r}=\pi w_{0}^{2} / \lambda_{0} \gg z_{t i p}$.

Wedge targets are modeled as two converging $100 \times n_{c r}$ plasma slabs with a stepfunction density profile, initial temperature $T_{e}, T_{i}=1 \mathrm{keV}$, ions of $M_{i}=3672 m_{e}$, and charge state $Z=1$. For subpicosecond timescales the $Z / A=1 / 2$ ratio here accurately reflects the ionization state of high-Z composition targets near the critical interface. In the region of laser irradiation system dynamics are dominated by collisionless processes, as $\nu_{e i} / \omega_{0} \sim 6 \times 10^{-2}$ for the Spitzer frequency $\nu_{e i}=$ $8 \pi n_{e} e^{4} \ln \Lambda /\left[3 \sqrt{3 m_{e}}(k T)^{3 / 2}\right][64]$. Resistive processes in the solid density target walls are neglected, as our analysis is primarily concerned with characterizing laser focusing and plasma ablation near the critical interface. The classical plasma skin depth at oblique incidence $l_{s} \approx c / \omega_{p} \cos \theta_{T}$ is resolved $\Delta z / l_{s} \sim 1$ for all targets, where the angle of incidence $\theta_{T}$ is the complement of the target opening half-angle. We have performed a separate test simulation with two times higher grid resolution in the $a_{3} \theta_{45}$ configuration and found no major qualitative differences.

\subsubsection{Characterizations of dynamical pulse-target evolution}

Fig. 5.4 depicts the evolution of several key LPI processes for the $a_{3} \theta_{17}$ pulse-target configuration. As shown in Fig. 5.2, laser focusing is most efficient in this geometry where focusing at peak intensity $I_{\text {peak }} / I_{0} \approx 8.8$ is achieved at time $t_{\text {peak }} \approx 350 \mathrm{fs}$ and sustained over 200 fs. Compared to an ideal optical case for $\theta_{17}$, i.e. an immobile ion target composition with $a \ll 1$ laser pulse as depicted in Fig. 5.1, this $I_{\text {peak }}$ represents $82 \%$ focusing efficiency. Peak laser focusing in this optical simulation is in agreement with results from the comparable $18^{\circ}$ target configuration reported on by Zeng et al. to within $13 \%$.[222] The inclusion of additional effects from self-consistent evolution of the target walls shows that laser focusing due to geometric optical compression is both attenuated by collisionless laser energy coupling into particles and boosted by 
nonlinear LPI processes. As the propagation channel fills with ablated underdense plasma, laser focusing is enhanced as the pulse rapidly enters the regime of relativistic self-induced transparency regime for $P_{L} \geq P_{c}=17\left(\omega_{0} / \omega_{p e}\right)^{2} \mathrm{GW}[46]$. At early time Fig. 5.4 (A) shows surface plasma with density exceeding $0.1 n_{c r}$ in the tip region ablated by collisionless laser absorption mechanisms. Brunel (vacuum) heating[24] is dominant here due to the oblique angle of incidence; its signature is further evident in Fig. $5.6(\mathrm{~A})$ at $1.4 \mathrm{ps}$ where hot electrons are bunched and driven through the target along the direction normal to its surface, with maximal energy flux density $P_{e} / I_{0} \approx 3.2$. In the higher intensity $a_{10} \theta_{17}$ pulse-target configuration, we observe identical values of $P_{e} / I_{0} \approx 3.2$ and $I_{\text {peak }} / I_{0} \approx 8.8$.

We identify a clear trend in the $\theta_{17}$ geometry where the region of peak laser intensity regresses away from the target tip as depicted in Fig. 5.3. While the magnitude of the the peak intensity $I_{\text {peak }} / I_{0}$ decays over hundreds of femtoseconds, the effective laser focal point recedes from the tip with intensity-dependent rates $u_{f} \equiv-d z_{\text {peak }} / d t \approx 0.037 c$ for $a_{1}$ and $u_{f} \approx 0.056 c$ for both pulses $a_{3}$ and $a_{10}$. Together with the constant $P_{e} / I_{0}$ and $I_{\text {peak }} / I_{0}$ ratios observed for $a>1$ laser pulses, this trend suggests the $\theta_{17}$ geometry may be representative of a broader class of configurations that are characterized by a sustained dynamical equilibrium between plasma expansion and laser radiation pressure.

Here we present a simple but general hydrodynamical model for pulse-target configurations in which a recession velocity with $\frac{d}{d t} u_{f}=0$ is observed. Consider an ion front moving at $u_{f}$ at the laser interface in planar geometry. The relevant conservation 


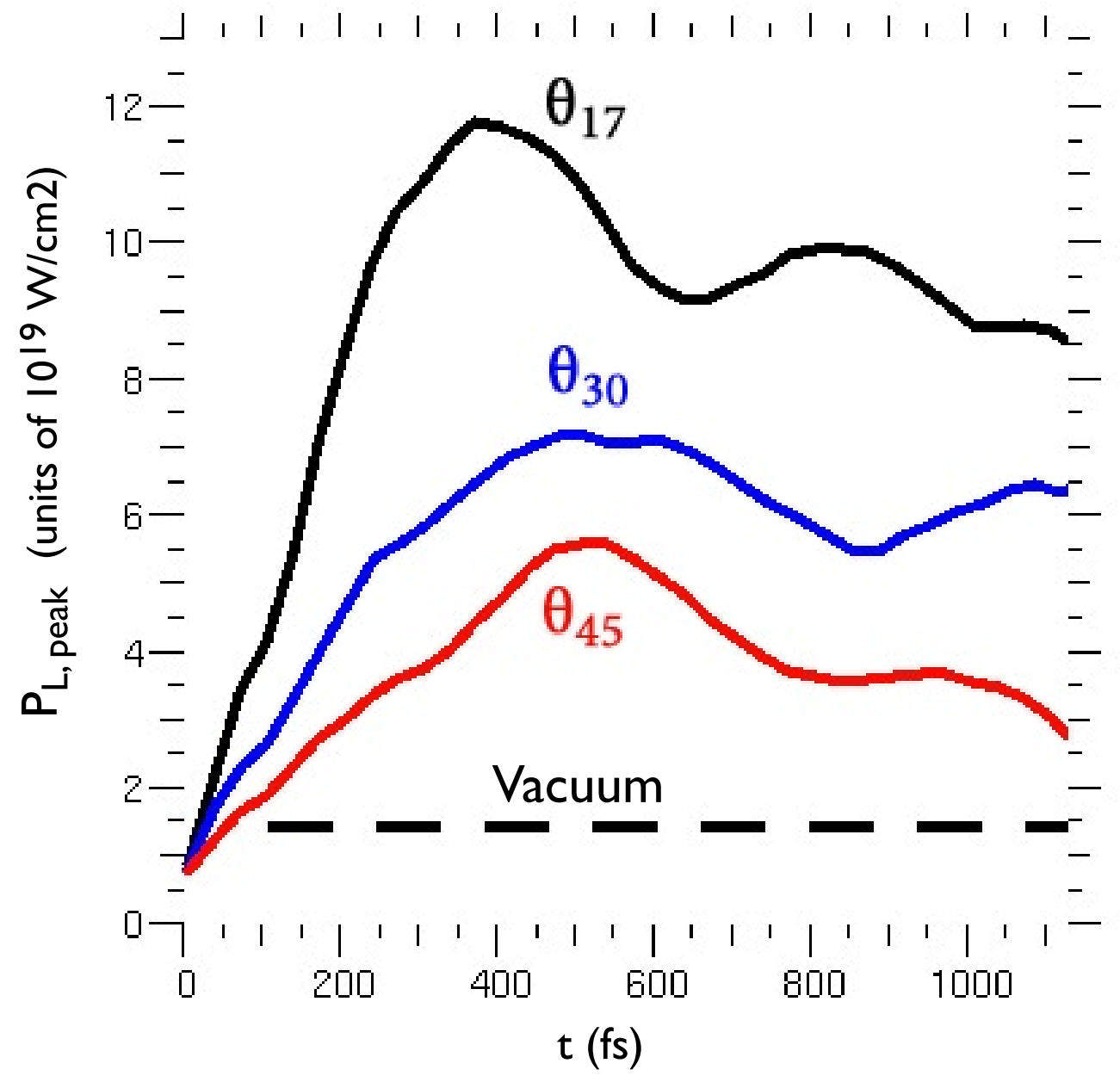

Figure 5.2 : Laser peak intensity dependence on target angle for the $a_{3}$ pulse (see text). 

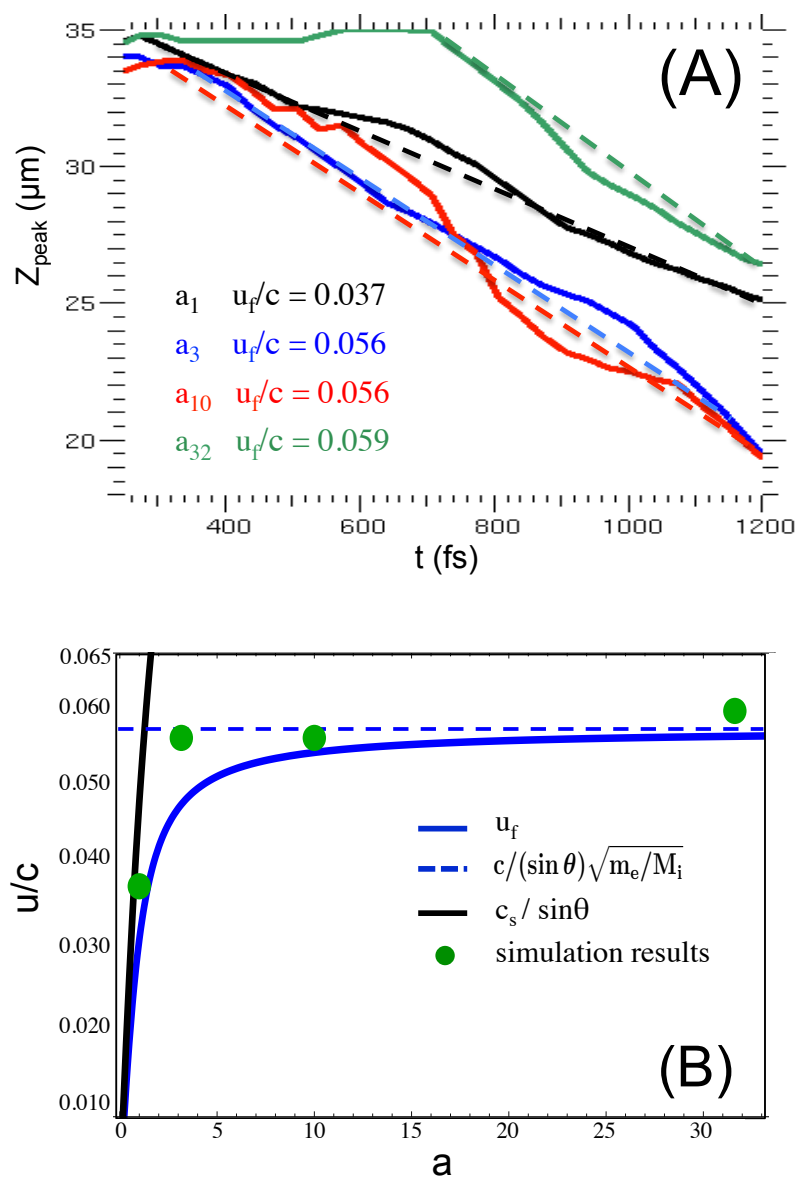

Figure 5.3: (A) Spatial location of peak laser intensity for the $\theta_{17}$ target; front velocity $u_{f}$ and time evolution of $z_{\text {peak }}$ (see text). (B) Scaling of $u_{f}$ with dimensionless laser parameter $a$ in $\theta_{17}$. The analytic model from equation (5.8) and its asymptotic form are represented by the solid and dashed blue curves respectively. 
equations are given by,

$$
\begin{aligned}
\partial_{t} \rho+\partial_{z}(\rho u) & =0 \\
\partial_{t}(\rho u)+\partial_{z}\left(\rho u^{2}+P\right) & =0
\end{aligned}
$$

where $\rho$ is the mass density. We now transform to a frame comoving with the ion front at $u_{f}$. In this frame, the front is stationary and two oppositely-directed flows of equal magnitude are present. By conservation of mass in equation (5.1) we see that the densities associated with these flows must be equal. Electrons in the critical interface are heated by the laser to $T_{e} \simeq T_{\text {pond. }}$ and particles are effectively trapped by $p_{z}<0$ plasma flowing into the laser channel. This quasi-isothermal interface contributes a plasma pressure term to the equation of motion and by equation (5.2) we may write,

$$
u_{f}^{2} M_{i} n_{i}=I / c-n_{i} T_{e}
$$

or,

$$
u_{f}=\left(u_{w}^{2}-c_{s}^{2}\right)^{1 / 2}
$$

where

$$
\begin{array}{r}
c_{s}=\left(T_{h} / M_{i}\right)^{1 / 2} \\
\frac{u_{w}}{c}=\left(\frac{I}{M_{i} n_{i} c^{3}}\right)^{1 / 2}=\left(\frac{Z m_{e} a^{2}}{2 \gamma M_{i}}\right)^{1 / 2}
\end{array}
$$

Here equation (5.3) assumes full reflection and $T_{e} \gg T_{i}$ in the interface. Equation (5.5) is the ion-acoustic velocity with the ponderomotive temperature scaling, and equation (5.6) represents the ion front velocity from Wilks et al.[215] 
Evaluating equation (5.4) using (5.5-5.6) yields,

$$
\frac{u_{f}}{c}=\left[\frac{m_{e}}{M_{i}}\left(1-\gamma^{-1}\right)\right]^{1 / 2}
$$

This expression represents the front velocity for planar geometry at normal incidence. In our converging target geometry, the front will recede towards the injection plane at a higher rate as the plasma flows over a shorter distance into the laser channel. Unbalanced by laser pressure, a straight-forward calculation shows the recession velocity for a converging target with angle $\theta, u_{f}=c_{s} / \sin \theta$ reaches $0.17 c$ for $a_{10}$. Transforming to the appropriate frame and solving for $u_{f}$ yields,

$$
\frac{u_{f}}{c}=\frac{1}{\sin \theta}\left[\frac{m_{e}}{M_{i}}\left(1-\gamma^{-1}\right)\right]^{1 / 2}
$$

As illustrated in Fig. 5.3 (B), equation (5.8) accurately predicts the front velocity and demonstrates the asymptotic form of $u_{f}$ for $a>3$ laser pulses. Results from an additional simulation performed at higher laser intensity $\left(I=1.37 \times 10^{21} \mathrm{~W} / \mathrm{cm}^{2}\right)$ are consistent with the asymptotic value of

$$
\frac{u_{f}}{c}=\frac{1}{\sin \theta}\left(\frac{m_{e}}{M_{i}}\right)^{1 / 2}
$$

as depicted in in Fig. 5.3. That the laser front feels zero net pressure over time implies a scaling $I_{\text {peak }}, P_{e} \propto a^{2}$ due to the strong correlation between the two variables for $a>1$. The nature of the coefficients here is determined by geometric, laser absorption and nonlinear focusing effects, and shall be the subject of examination in future work. In the context of $K_{\alpha}$ radiography this unique class of wedge targets could be 
employed to achieve tightly-focused spots with order-of-magnitude higher intensity than the incident beam, scaling to intensities beyond $a>10$ without degradation in performance.

In the $a \leq 1$ regime, it is expected that laser focusing will be enhanced due to sharply reduced absorption, e.g. by $28 \%$ for vacuum heating for $a_{1}$ versus $a_{3}$ using Brunel's 1-D analytical model[24], while the critical power threshold for self-focusing in the underdense region is still readily achieved. This is in good agreement with the results obtained for the $a_{1}$ pulses, where we see higher peak focusing by a factor of 1.5 on average across geometries. In this regime the laser is not sufficiently relativistic to excite electrons up to $\mathrm{MeV}$ energies; at intensity $a_{1}, T_{h}=0.2 \mathrm{MeV}$ and we accordingly observe that $P_{e}=0$ across geometries. By contrast, electron energy flux densities in the center of Fig. 5.6 (A) represent the convolution of several heating mechanisms that operate efficiently in the underdense plasma upstream of the target tip. SM LWFA, a process where the laser self-interaction leads to longitudinal modulations and electron bunching at $\omega_{\text {pe }}$, plays the dominant role in particle heating here. For the inhomogenous underdense plasma present in Fig. 5.4 (B-C), the longitudinal electric field generated in this mechanism produces a broad continuous electron temperature spectra ranging from keV to hundreds of MeV[46]. The inverse free electron process also contributes to particle heating; in this mechanism quasistatic electric and magnetic fields in the laser channel induce transverse betatron oscillations that couple laser energy into electrons when the betatron frequency $\omega_{\beta} \approx \omega_{p e} / 2 \gamma^{1 / 2}$ nears resonance with the optical frequency in the frame of the relativistic electron[166]. The signature of this process is present in Fig. $5.6(\mathrm{~A})$ where we observe longitudinal bunching of electrons at $2 \omega_{p e}$ with temperature scaling approximately three times higher than ponderomotive. In the $a_{3} \theta_{17}$ and $a_{3} \theta_{30}$ pulse-target configurations, electron dynamics at late times are characterized by the presence of a 

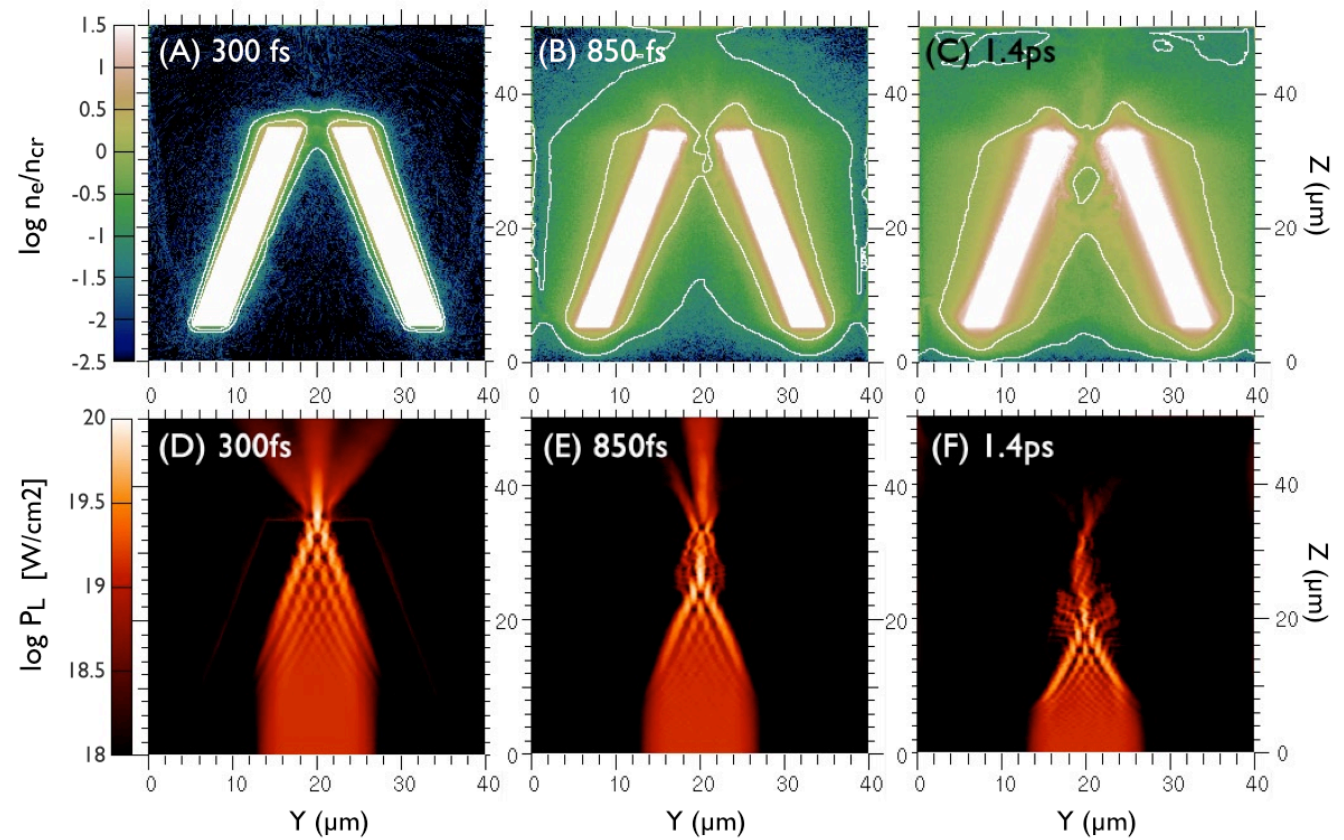

Figure 5.4: Temporal evolution of the $a_{3} \theta_{17}$ pulse-target configuration in $Y-Z$ space. (A-C) Time-averaged electron density $\log n_{e} / n_{c r}$ with superimposed white contour lines corresponding to densities of $0.1 n_{c r}$ and $n_{c r} ;(\mathrm{D}-\mathrm{F})$ time-averaged laser Poynting flux $\log P_{L}\left[W / \mathrm{cm}^{2}\right]$.

dominant hot electron filament aligned with the target tip. For these configurations $P_{e} / I_{0} \approx 3.2,1$ respectively, as depicted in Fig. $5.6(\mathrm{~A}-\mathrm{B})$ with scalings to higher intensity in $\theta_{17}$ described above. The potential viability of these energetic collimated electron structures for particle beam systems will be discussed briefly in the next section.

For the $a_{3} \theta_{30}$ configuration, plasma ablation in the critical interface is comparable to $a_{3} \theta_{17}$, with focusing efficiency attenuated to $I_{\text {peak }} / I_{0} \approx 5.2$ as shown in Fig. 5.2. This peak is shifted to later time by 100 fs compared to $a_{3} \theta_{17}$ due to the longer light propagation time and interaction region associated with the wider target angle. The $a_{3} \theta_{45}$ configuration at late times is characterized by $n_{c r}$ plasma in the tip 
region and significant laser filamentation. This filamentation, coupled with the large spatial width of the target, suppresses ponderomotive self-channeling and results in effective closure of the target tip. The diminished peak focusing $I_{\text {peak }} / I_{0} \approx 3.7$ is again shifted out to later time by $\approx 150 \mathrm{fs}$ as seen in Fig. 5.2 by which time the tip has already started to close. Fig. 5.5 illustrates the 11 times higher laser energy coupling into the target walls at $300 \mathrm{fs}$ in this configuration compared to $a_{3} \theta_{17}$. This absorption has the effect of dephasing the laser light and, together with the lower geometrical compression associated with wider targets, plays an important role in limiting the attainable peak laser focusing in the $\theta_{30}$ and $\theta_{45}$ geometries. For the $a_{10} \theta_{30}$ configuration, peak focusing of $I_{\text {peak }} / I_{0} \approx 6.9$ is sustained over a 600 fs interval prior to tip closure at $t \approx 1.1 \mathrm{ps}$ due to the attenuated ponderomotive force associated with laser filamentation. In addition we find electron acceleration in the tip region in this configuration to be effectively suppressed. As depicted in Fig. 5.6 (C) the $a_{3} \theta_{45}$ pulse-target configuration exhibits two equally dominant hot electron filaments off to the sides of the tip with negligible electron acceleration through the tip. These structures are spatially coincident with strong filaments in the laser Poynting flux. This description is also representative of hot electron generation for $a_{10} \theta_{45}$. In these configurations, laser filamentation coupled with the large transverse width of the target suppresses laser pressure and the tip effectively closes.

To the extent that assumptions underpinning the relativistic laser absorption model developed in sections 3.1 and 3.5 are maintained, the absorption of laser light and its conversion into the kinetic energies of directed plasma flows in this situation is subject to the bounds given by equation (3.72). Since the model was derived in a manner that is insensitive to the precise mechanisms of electron acceleration, a set of processes that changes elements with angle of incidence as covered above, its predictions apply to this situation at early times. Yet as the hot plasma expands away 

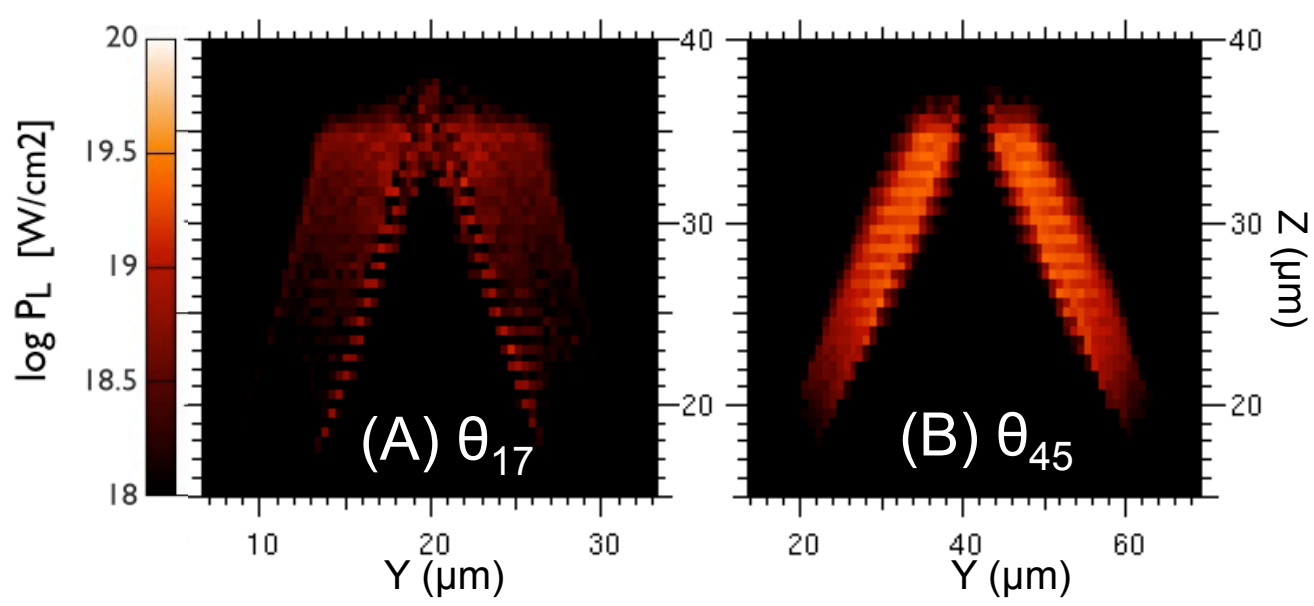

Figure 5.5 : Laser energy absorption into target walls at 300 fs for the $a_{3}$ laser pulse (note different transverse scales).

from the surfaces of the focusing wedges over time, the wedge tip begins to close as absorption takes place primarily in underdense regions near the midplane. Dynamical evolution of the plasma consistent with this description is shown in Fig. 5.4 (A-C). Hence, absorption exceeding the upper bound given by equation (3.72) is possible, and the tip of the wedge is observed to close. While direct comparison with the absorption theory is not practical since the relevant simulation data is unavailable, future work will focus on extending the theory's domain to underdense petawatt-laser plasma interactions, as is covered in section 3.5.

In this section we have employed two dimensional particle-in-cell simulations to study the focusing and evolution of ultraintense short pulse lasers in idealized converging targets. The wide $45^{\circ}$ targets are found to exhibit generally suboptimal performance over time; for the $I_{19}$ and $I_{20}$ pulses, particle heating is characterized by two equally dominant hot electron filaments off to the sides of the tip. Ponderomotive self-channeling is suppressed by laser filamentation and the target tip effectively 


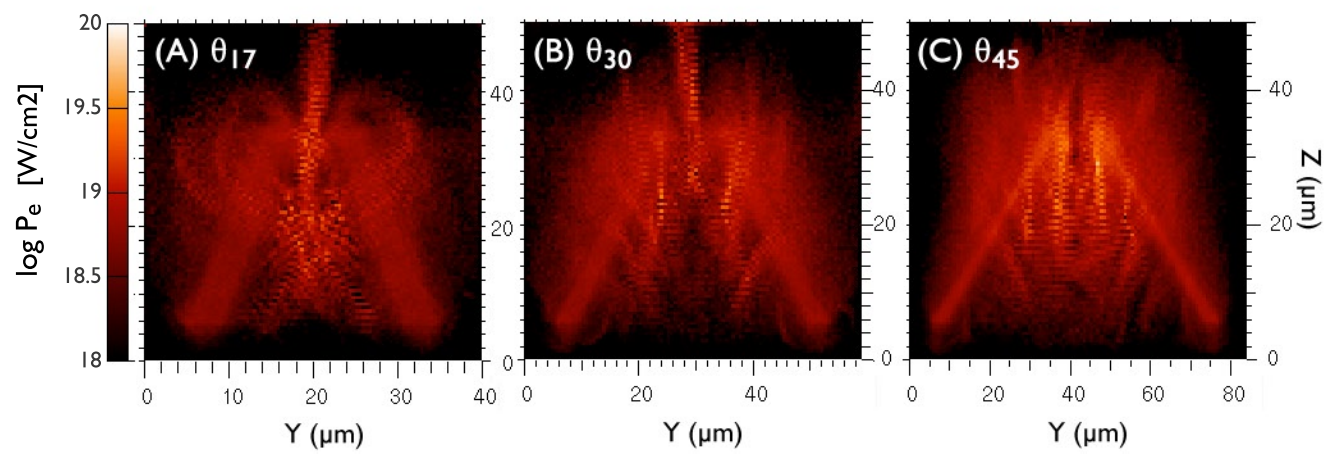

Figure 5.6 : Hot electron characteristics at 1.4 ps across target geometries for the $a_{3}$ laser pulse; energy flux densities of forward-going electrons $\log P_{e}\left[\mathrm{~W} / \mathrm{cm}^{2}\right]$ (note different transverse scales).

closes at $t \approx 450$ fs. In the $30^{\circ}$ geometry performance degrades with increasing intensity; for the $I_{19}$ pulse, peak laser focusing of $I_{\text {peak }} / I_{0} \approx 5.2$ and the generation of tip-aligned electron filaments with $P_{e} / I_{0} \approx 1$ are observed. In the $I_{20}-30^{\circ}$ configuration, sustained peak focusing of $I_{\text {peak }} / I_{0} \approx 6.9$ is present at early times, with electron acceleration in the tip region suppressed as the tip closes at $t \approx 1.1 \mathrm{ps}$. In general, performance in these wider targets is limited by large interaction regions and high absorption in the overdense walls that act to dephase laser light, diminished geometrical compression with respect to the $17^{\circ}$ target, and instabilities such as laser filamentation that deleteriously affect ponderomotive self-channeling.

By contrast, in the $17^{\circ}$ configuration both peak laser focusing of $I_{\text {peak }} / I_{0} \approx 8.8$ and electron generation and propagation through the target tip with $P_{e} / I_{0} \approx 3.2$ are achieved for $a>1$. This peak focusing represents $82 \%$ efficiency relative to an ideal optical case, comparable to results reported on previously[222], with losses primarily going into hot electron excitation. A clear trend is described for this geometry where the region of peak laser intensity regresses away from the target tip at intensitydependent rates that saturate for pulses of $a>3$. This trend suggests the $17^{\circ}$ 
geometry may be representative of a broader class of pulse-target configurations that are characterized by a sustained dynamical equilibrium between plasma expansion and laser radiation pressure. The physical picture here centers on the interface between the receding laser front and the plasma expansion. The onset of recession occurs with a density perturbation in this region at early time, and as the plasma expands ablated particles stream in to the laser channel. Over time the laser energy density gradient becomes gentler, outward ponderomotive pressure decreases, and the laser front regresses towards injection plane. A simple but general model is developed that encapsulates the essential character of these systems which accurately predicts $u_{f}$ and yields key scalings $I_{p e a k}, P_{e} \propto a^{2}$. For the parameters considered here, the hydrodynamic plasma expansion breaks down beyond subpicosecond timescales after which the high collisionality, ionization and resistive effects that dominate deep in realistic high-Z composition target walls must be included. Future research will focus on expanding the applications of our model to a broader set of configurations, and on analytically identifying pulse-target configurations in this dynamical equilibrium between plasma expansion and ponderomotive cavitation. Our results suggest the $17^{\circ}$ and other configurations of this class may be employed as an experimentally straight-forward apparatus to achieve bursts of 10x boosted light for hundreds of femtoseconds, focused down to small spots of $\sim 1 \lambda_{0}$ with high performance scaling beyond intensities $a>10$.

In addition the results presented here may have relevance for production cone target environments such as fast ignition. Although our simulation geometry is Cartesian and therefore "slab," this is a reasonable facsimile of the physics that will occur for conical targets in $R-Z$ space. Assuming a linear scaling for geometrical compression and given laser $p$-polarization, focusing for these configurations in three dimensions is enhanced by an additional factor of 4 . In the $17^{\circ}$ geometry this corresponds to 
a $~ 35$ times higher intensity spot in a conical target with a collimated, tip-aligned hot electron filament for $a>1$ laser pulses over $\sim 500$ fs. Though electron transport in the dense thermonuclear fuel remains a key outstanding issue in the FI literature, the enhanced laser focusing described in this section could in principle allow ignitor pulse requirements to met near $I_{19}$. Furthermore, for a given amount of input laser energy, these configurations could enable an additional control parameter to tune beam intensity at the tip of target. If higher intensity pulses are required, the saturation of $u_{f}$ potentially enables current cone-wire experiments[149] to utilize narrow targets for gains in laser focusing while suffering no additional penalty in laser-fuel payload distance. As these results for the $17^{\circ}$ geometry present a focusing scheme enabling laser intensity amplification by a factor of ten, in a manner that is intensity-indepenedent, this scheme could support reaching the QED-plasma regime using sub-quantum critical field laser light as an input. 


\section{Chapter 6}

\section{Development of interpretative proton radiography simulation tool for HED plasmas}

Proton radiography is a useful diagnostic of HED plasmas under active theoretical and experimental development. In this chapter we describe a new simulation tool that interacts realistic laser-driven point-like proton sources with three dimensional electromagnetic fields of arbitrary strength and structure and synthesizes the associated high resolution proton radiograph. The present tool's numerical approach captures all relevant physics effects, including effects related to the formation of caustics. Electromagnetic fields can be imported from PIC or hydrodynamic codes in a streamlined fashion, and a library of electromagnetic field 'primitives' is also provided. This latter capability allows users to add a primitive, modify the field strength, rotate a primitive, and so on, while quickly generating a high resolution radiograph at each step. In this way, our tool enables the user to deconstruct features in a radiograph and interpret them in connection to specific underlying electromagnetic field elements. We show an example application of the tool in connection to experimental observations of the Weibel instability in counterstreaming plasmas, using $\sim 10^{8}$ particles generated from a realistic laser-driven point-like proton source, imaging fields which cover volumes of $\sim 10 \mathrm{~mm}^{3}$. Insights derived from this application show that the tool can support understanding of HED plasmas. 


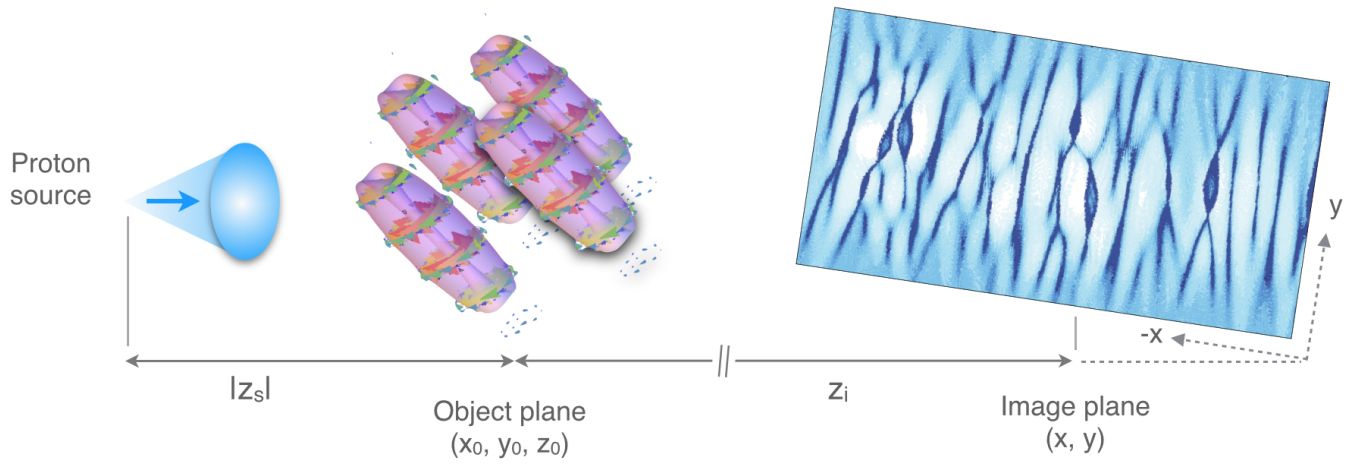

Figure 6.1 : Schematic of key aspects of the proton radiography simulation tool, following the propagation of protons along the $z$ axis from left to right. Parameters controlling the proton source are described in section 6.1.2. As an example, specifying the source control vector $\vec{S}=\left(2,14.7 \mathrm{MeV}, 10^{9}, 1 \mathrm{~cm}\right)$ creates a 14.7 MeV monoenergetic proton source isotropically emitting 1 billion protons, imitating a realistic D ${ }^{3} \mathrm{He}$ source, situated $\left|z_{s}\right|=1 \mathrm{~cm}$ from the object plane containing the plasma electromagnetic fields. Depicted in the object plane at $z=0$ are four tilted ellipsoidal magnetic filaments, each having form given by equation (6.3). These fields are created in PRIME by specifying the single field control vector $\vec{L}=(4,2,2,100 \mu \mathrm{m}, 100 \mu \mathrm{m}, 50 \mu \mathrm{m}, 500 \mu \mathrm{m}, 0,0,1 \mathrm{MG})$, as described in section 6.1.1. A simulated proton radiograph created by the tool is shown in the image plane, situated at $z=z_{i}$. Details on the field structure underpinning this radiograph are covered in section 6.3. 


\subsection{Features of PRIME}

PRIME is a three dimensional simulation tool that we have been developing for modeling HED plasma situations. Both realistic TNSA and $\mathrm{D}^{3} \mathrm{He}(14.7$ and $3 \mathrm{MeV})$ laser-driven proton sources have been tested in experimentally-realistic configurations and are available to the user. Additionally the user has the ability to specify a proton source having arbitrary spectral properties. We anticipate that this radiography tool will have two primary uses. The first is in constructing electromagnetic field structures using primitives, guided by the predictions of plasma physics theory and PIC and hydrodynamic simulation results. This approach provides the advantage that fields are free of numerical noise, a key issue arising in kinetic simulations of mm-scale

plasmas. Here the user also has the capability to add a primitive, modify the field strength, rotate a primitive, and so on, while quickly generating a high resolution radiograph at each step. In this manner PRIME should provide insights into the crucial question of how to interpret proton radiographs. We also anticipate that synthetic radiographs produced by this tool should become particularly useful in cases where running PIC and hydrodynamic codes is computationally infeasible, and further to guide these expensive simulations toward larger scale. The second use of this tool will be in quickly and efficiently simulating a high resolution proton radiograph associated with electromagnetic fields exported from PIC and hydrodynamic codes. For this purpose we have built in the capability to import fields directly from a variety of existing codes (e.g. OSIRIS[55]).

Related to the first use, the standard object description in PRIME is a three dimensional electromagnetic primitive describing the volumetric field structure. The user has a number of high level options for inputting these fields, for example generating a lattice of primitives or programmatically including randomization effects, 
that are enumerated in section 6.1.1. By combining primitives together the user can simulate fields representative of a large number of important HED processes including electrostatic planar shock waves, magnetized cylindrical shocks, two-stream and other electrostatic instabilities, intense laser-driven $\nabla n \times \nabla T$ 'Biermann battery' magnetic fields (for plasma density $n$ and temperature $T$ ) and filamentary magnetic field structures generated via the Weibel instability[97, 77, 188, 96].

With respect to numerical schemes, in PRIME we have implemented a modular approach in order to accurately and efficiently simulate the proton radiography technique. This is motivated by the disparate spatial scales characterizing the source plasma - detector system. The macroscopic volume is vast: the detector typically sweeps out an area $\sim 25 \mathrm{~cm}^{2}$ and the axial distance between the source and detector, passing through the interaction region containing the HED plasma, can exceed $>10 \mathrm{~cm}$. At the same time the microscopic field structures associated with the plasma often have spatial scales of $\sim \mu \mathrm{m}$. Simulating the full volume of the cone connecting the source to the detector resolving the electromagnetic fields would require $\sim 10^{14}$ grid cells. This situation clearly exceeds reasonable computational efforts. Therefore to mitigate this issue in PRIME we have divided the system into three regions. The tool covers the source-to-plasma object region, region containing the plasma object itself, and plasma object-to-detector region, as well as the interfaces connecting them. In the plasma region we are currently using LSP[211] for the particle push. This provides the additional advantage that scattering models for dense plasmas as well as deflections due to electromagnetic forces can be included. The modular approach in PRIME allows a set of electromagnetic fields to be specified, then different proton sources and different detectors to be 'hooked up' to these fields in a streamlined manner. For example in section 6.3 we show several high resolution proton radiography results of filamentation-instability-driven fields, obtained by keeping the fields 
unchanged while swapping between realistic proton sources. By allowing users to quickly image the same field configuration using a TNSA proton source, and $3 \mathrm{MeV}$ and $14.7 \mathrm{MeV} \mathrm{D}{ }^{3} \mathrm{He}$ proton sources, we show that PRIME can help unravel the convolution between the properties of the source and those of the electromagnetic fields. The particle push and other parts of the code have been parallelized in order to take advantage of the Lawrence Livermore National Laboratory (LLNL) Livermore Computing (LC) Linux architecture, enabling efficient radiography simulations.

\subsubsection{Tools for constructing electromagnetic fields}

A robust set of tools is available to the user for constructing electromagnetic fields in PRIME . The complete library of analytic electromagnetic field primitives described in [97] is available to the user, including electrostatic Gaussian ellipsoids, ambipolar electric field structures, magnetic flux ropes and magnetostatic Gaussian ellipsoids. The associated functional forms are enumerated in section 6.2. While the length scales of the primitives set the grid resolution, the particle push timestep is adjusted to the Courant condition[12] evaluated using the velocity of the protons, enabling efficient and fast simulations. Each primitive is controlled by a set of parameters governing the peak electric (magnetic) field strength $E_{0}\left(B_{0}\right)$, the Cartesian position of the primitive's centroid $\left(x_{0}, y_{0}, z_{0}\right)$ with respect to the center of the region containing the HED plasma and two angles $\theta$ and $\psi$ indicating the primitive's polar and azimuthal angles relative to the proton propagation axis $\hat{z}$. The sign of $E_{0}\left(B_{0}\right)$ determines whether protons interacting with the primitive will experience a focusing $\left(E_{0}, B_{0}>0\right)$ or defocusing force. Spatial extent is specified, taking the ellipsoids for example, using the parameters $a$ and $b$ representing the major and semi-major axes respectively. By varying the ratio $a / b$ the user can produce field structures representative of Weibel instability-driven magnetic filaments, as well as advecting laser-driven 
Biermann battery-like magnetic 'pancakes' [98, 96, 188].

The user can construct a field topology featuring an arbitrary number of primitives, each having unique parameters. A number of input methods describing configurations of several primitives are available to the user. At the lowest level, the user specifies a list of $N$ field control vectors each having the form,

$$
\vec{G}_{n}=\left(\mathcal{P}, x_{0}, y_{0}, z_{0}, \theta, \psi, a, b, E_{0}\left(B_{0}\right)\right)
$$

which are then transformed by the tool into $N$ volumetric fields in the three dimensional simulation $(n \in[1, N])$. The $\mathcal{P}$ element is an integer mapping to the desired primitive type ( $\mathcal{P}=1$ corresponds to an electrostatic Gaussian ellipsoid for example). In the simulation overlapping regions of field have $E$ and $B$ automatically summed.

Higher level input options are also available to the user. To support modeling of periodic systems, a lattice of primitives can be generated programmatically by specifying a single field control vector of the form,

$$
\vec{L}=\left(\mathcal{P}, N_{\text {rows }}, N_{\text {cols }}, d_{\text {rows }}, d_{\text {cols }}, a, b, \theta, \psi, E_{0}\left(B_{0}\right)\right)
$$

The tool transforms this vector into a body-centered rectangular prism lattice of $N_{\text {rows }} \times N_{\text {cols }}$ primitives of type $\mathcal{P}$. The lattice is centered at the origin of the plasma region and the $n^{\text {th }}$ primitive has the centroid position $\left(x_{0, n}, 0, z_{0, n}\right)$. Rows are oriented along $\hat{z}$ and columns are oriented along $\hat{x}$. $N_{\text {rows }}$ is thus the number of primitives in the lattice in $\hat{z}$ and $d_{\text {rows }}$ is the spacing between primitives in $\hat{z}$. Similarly the 'cols' subscript corresponds to periodicity in $\hat{x}$.

To support more realistic field configurations, high level input options that enable randomization effects are also available to the user. By appending the elements 
$\left(\delta \theta, \delta \psi, \delta E_{0}\left(\delta B_{0}\right)\right)$ to the lattice-generating vector $\vec{L}$, the user can programmatically make unique the $\theta, \psi$ and $E_{0}\left(B_{0}\right)$ values for each primitive. Taking the altitude angle as an example, specifying $\delta \theta=0$ (or omitting the $\delta$ elements) means that $\theta_{n}=\theta$ for the $n^{\text {th }}$ primitive. Randomization effects enter as specifying a nonzero $\delta \theta$ applies the mapping $\theta_{n} \rightarrow \theta+\delta \theta_{n}$, where $\delta \theta_{n} \in[-\delta \theta, \delta \theta]$ is sampled randomly within this interval for each primitive. Individualized parameter effects can be as small or large as desired, and are generally quite important since they introduce a realistic asymmetry into the simulation. Indeed, section 6.3 below discusses the significant impact on the resulting proton radiographs of $\delta \theta$ and $\delta \psi$ effects in representing filamentationinstability-driven fields.

\subsubsection{Specifying source and detector properties}

Two methods of proton beam generation are supported, which together offer users the capability to specify sources with arbitrary spectral properties. The first method produces point proton sources. The user can choose a temperature $T_{p}$ representing TNSA-generated protons having a quasi-Maxwellian distribution. Alternatively, with the first method users can specify an energy $\mathcal{E}_{p}$ to generate a mononergetic point proton source. Specifying $\mathcal{E}_{p}=3$ or $14.7 \mathrm{MeV}$ reproduces the properties of protons generated through fusion reactions in intense laser-imploded $\mathrm{D}^{3} \mathrm{He}$ capsules. In addition to setting the energy parameter, users also choose the number of protons to simulate $N_{p}$ and the axial position of the source, $z_{s}<0$, relative to the object plane containing the HED plasma at $z_{o}=0$. The dimensions of the plasma region $\ell_{x}, \ell_{y}$ and $\ell_{z}$ (lengths in $x, y$ and $z$ respectively) are determined automatically such that they contain the plasma. This region is centered at $(0,0,0)$ and is situated between $|x| \leq \ell_{x} / 2,|y| \leq \ell_{y} / 2$ and $|z| \leq \ell_{z} / 2$. The proton source is then instantiated in the simulation at the position $\left(0,0, z_{s}\right)$ with a phase space distribution corresponding 
to a point source according to these parameters. In short, a realistic point proton source is created in PRIME by specifying a single source control vector of the form $\vec{S}=\left(\mathcal{S}, T_{p}\left(\mathcal{E}_{p}\right), N_{p}, z_{s}\right)$, where $\mathcal{S}=1$ and the second element is $T_{p}$ for a TNSA source or $\mathcal{S}=2$ and the second element is $\mathcal{E}_{p}$ for a monoenergetic source.

In the second proton generation method, the user specifies the proton source 'spot' size $r_{p}$ in addition to $N_{p}$ and $z_{s}$. The source is then instantiated in the simulation at $z=z_{s}$ with finite transverse size between $x^{2}+y^{2} \leq r_{p}^{2}$. The proton beam divergence and energy distribution are specified through a combination of the beam thermal temperature $T_{p}$ and a vector drift velocity $\vec{V}_{p}$. The user can specify spatial variations in both $T_{p}(x, y)$ and $\vec{V}_{p}(x, y)$ across the source. This allows a high degree of customization of beam properties, for example reproducing a plane proton source when $T_{p}=0$ and $\vec{V}_{p}(x, y)=(0,0$, const. $)$.

To support a range of conditions, the user has the option to specifiy the detector properties in addition to the source properties. The user may choose the image plane axial position $z_{i}$ of the detector corresponding to the nominal magnification $M=-z_{i} / z_{s}$. The user also can specify the size of the detector and the binning resolution in each transverse direction. If no detector attributes are chosen, the default detector will be instantiated in the simulation with infinite transverse dimensions at $z_{i}=10 \mathrm{~cm}$, with $30 \mu \mathrm{m} \times 30 \mu \mathrm{m}$ resolution in nominal object plane units.

\subsection{Benchmarking against analytic theory}

Analytic theory describing the connection between electromagnetic fields and the fluence images produced by sampling protons has been developed in Kugland et al.[98] In this section predictions of this formalism are compared to results produced by our numerical radiography tool. 

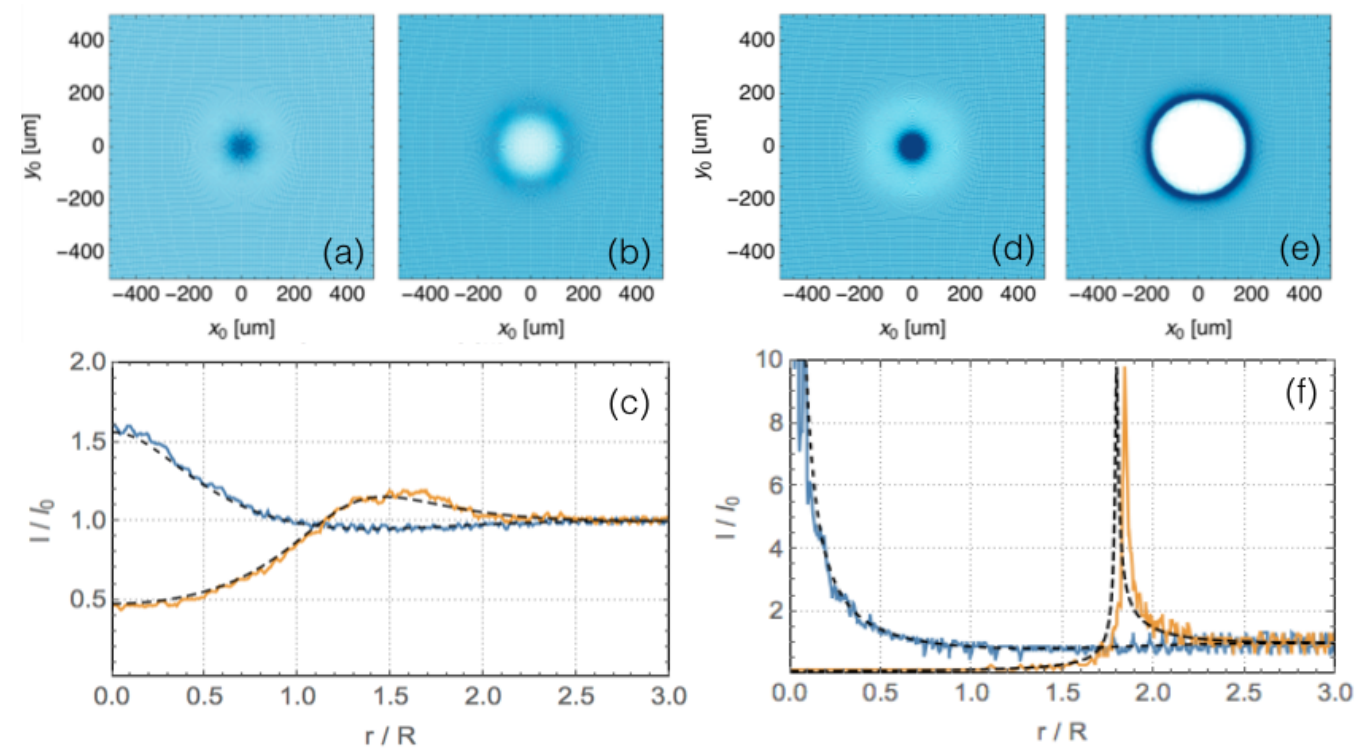

Figure 6.2 : Comparison between theory and simulation results in the linear regime. The situations shown here interact a monoenergetic $\mathcal{E}_{p}=14.7 \mathrm{MeV}$ proton source of $\left|z_{s}\right|=1 \mathrm{~cm}, z_{i}=10 \mathrm{~cm}$ with a single magnetic filament of the form given by equation (6.3) having $a=100 \mu \mathrm{m}$ and $b=300 \mu \mathrm{m}$. (a-b) show simulated radiograph results using $B_{0}=0.2 \times B_{0 \text { crit }}$ for the focusing and defocusing cases, respectively, while (d-e) use $B_{0}=0.9 \times B_{0 \text { crit }}$. The color scale is fixed between images with darker (lighter) regions indicating a surplus (deficit) of protons. (c) and (f) depict normalized lineouts of the proton fluence along $y_{0}=0$ for the $0.2 \times B_{0 \text { crit }}$ and $0.9 \times B_{0 \text { crit }}$ simulations, respectively. The blue curves correspond to the focusing cases and the yellow curves to the defocusing cases. The simulations agree with the theory predictions of equations (6.13-6.14), indicated using dashed black curves, to better than $5 \%$ in all cases. 
Consider a Gaussian ellipsoidal 'cocoon' filled with magnetic field having only an azimuthal $(\varphi)$ component,

$$
B_{\varphi}=B_{0} \frac{r}{a} \exp \left(-\frac{r^{2}}{a^{2}}-\frac{z^{2}}{b^{2}}\right)
$$

for radial coordinate $r$, axial coordinate $z$ and semi-major and major axes $b$ and $a$, respectively. For elongated $b>a$ situations this field structure resembles a single Weibel instability-driven magnetic filament[97, 96, 188]. Note that in this representation $B_{0}$ is not a maximum value of the field; the maximum is reached at $r=a / \sqrt{2}$ and is equal to $B_{\text {peak }}=B_{0} / \sqrt{2 e} \approx 0.43 B_{0}$ where $e$ is the natural logarithm base. To create this primitive in the radiography tool the user specifies the index $\mathcal{P}=4$ in conjunction with equation (6.1). We assume that the distance from the source to the center of the object is $\left|z_{s}\right|=1 \mathrm{~cm}$, the distance from the center to the image plane is $z_{i}=10 \mathrm{~cm}$, proton energy is $\mathcal{E}_{p}=1 / 2 m_{p} v_{p}^{2}=14.7 \mathrm{MeV}$ for proton mass $m_{p}$ and velocity $v_{p}, a=100 \mu \mathrm{m}$ and $b=300 \mu \mathrm{m}$.

This situation is therefore consistent with the paraxial approximation $\left(a /\left|z_{s}\right| \sim\right.$ $\left.10^{-2}\right)$. In the analytic evaluation of the proton deflection we use the smallness of the dimension $b$ compared to the proton gyroradius $\rho \sim 3 \mathrm{~cm}$ for the fields that are needed to form the caustics. This allows us to use a linear approximation: integration of the transverse force over the unperturbed (straight) trajectory within the field structure. The anticipated error of this assumption is less than 10\%. With that, we find that deflection angle $\alpha$ is related to the radius $r_{0}$ of the point where protons intersect the object plane by,

$$
\alpha=\mu \frac{r_{0}}{a} \exp \left(-\frac{r_{0}^{2}}{a^{2}}\right)
$$


where

$$
\mu=\frac{\sqrt{\pi}|e| B_{0} b}{m_{p} v_{p} c}
$$

is a dimensionless parameter characterizing the interaction and $e$ is the fundamental charge. For the $14.7 \mathrm{MeV}$ proton source $v_{p} / c=0.177$ and $\mu=3.2 \times 10^{-6} B_{0}[\mathrm{~T}] b[\mu \mathrm{m}]$. The position of the point in the image plane is determined by,

$$
r=z_{i}\left(-\frac{r_{0}}{z_{s}} \mp \alpha\left(r_{0}\right)\right)
$$

where the sign 'minus' corresponds to the focusing case and the sign 'plus' to a defocusing case. The derivative $d r / d r_{0}$ is,

$$
\begin{aligned}
\frac{d r}{d r_{0}} & =-\frac{z_{i}}{z_{s}}\left[1 \mp \frac{\mu\left|z_{s}\right|}{a} f\left(r_{0} / a\right)\right] \\
f\left(r_{0} / a\right) & =\left(1-2 \frac{r_{0}^{2}}{a^{2}}\right) e^{-r_{0}^{2} / a^{2}}
\end{aligned}
$$

For small $\mu$ (small magnetic field) the second term is negligible and one has just a uniform magnification. When one increases $\mu$, the condition $d r / d r_{0}=0$ is finally met at some $\mu_{\text {crit }}$ having different values for the focusing and defocusing cases. For the focusing case the critical value is,

$$
\mu_{\text {crit }}=-\frac{a}{z_{s}}
$$

whereas for the defocusing case,

$$
\mu_{\text {crit }}=-\frac{a}{z_{s}} \frac{e^{3 / 2}}{2} \approx-2.24 \frac{a}{z_{s}}
$$


Introducing values of the universal constants one arrives at the following expressions for the critical magnetic fields,

$$
B_{0 \text { crit }}[\mathrm{T}]=-8.12 \frac{a}{b} \frac{\sqrt{\mathcal{E}_{p}[\mathrm{MeV}]}}{z_{s}[\mathrm{~cm}]}
$$

and

$$
B_{0 \text { crit }}[\mathrm{T}]=-18.2 \frac{a}{b} \frac{\sqrt{\mathcal{E}_{p}[\mathrm{MeV}]}}{z_{s}[\mathrm{~cm}]}
$$

for the focusing and defocusing cases, respectively. Using the input parameters for these test cases, we find the fields of $10.38 \mathrm{~T}$ and $23.26 \mathrm{~T}$, respectively.

Using equations (6.4-6.8) the intensity distribution in the image plane for $\mu$ smaller than critical can be presented in parametric form as,

$$
\begin{aligned}
& \frac{I}{I_{0}}=\left|e^{-2 t^{2}}\left(\nu \mp e^{t^{2}}\right)\left(e^{t^{2}} \mp \nu\left(1-2 t^{2}\right)\right)\right|^{-1} \\
& \frac{r}{R}=t\left|1 \mp \nu e^{-t^{2}}\right|, \quad \nu \equiv-\frac{\mu z_{s}}{a}
\end{aligned}
$$

for parameter $t$. Here $I_{0}$ is the intensity in the center of the image plane in the absence of an object and $R=-z_{i} a / z_{s}$. These equations follow from the definition of the object-image coordinate relationship, i.e., $r=r_{0}+z_{i} /\left|z_{s}\right| r_{0}+\alpha z_{i}$ for spatial coordinate $r$, according to equation (1) of Kugland et al.[98] The intensity expressions follow from the Jacobian describing the object-image coordinate transformation from equations (8) and (12) in ref. [98].

One can also plot intensity distributions for the fields exceeding critical values. In order to do so the amplitude-limiting factor $\epsilon$ as described in ref. [98] must be accounted for in equation (6.13). The appropriate parametric relation for the nor- 

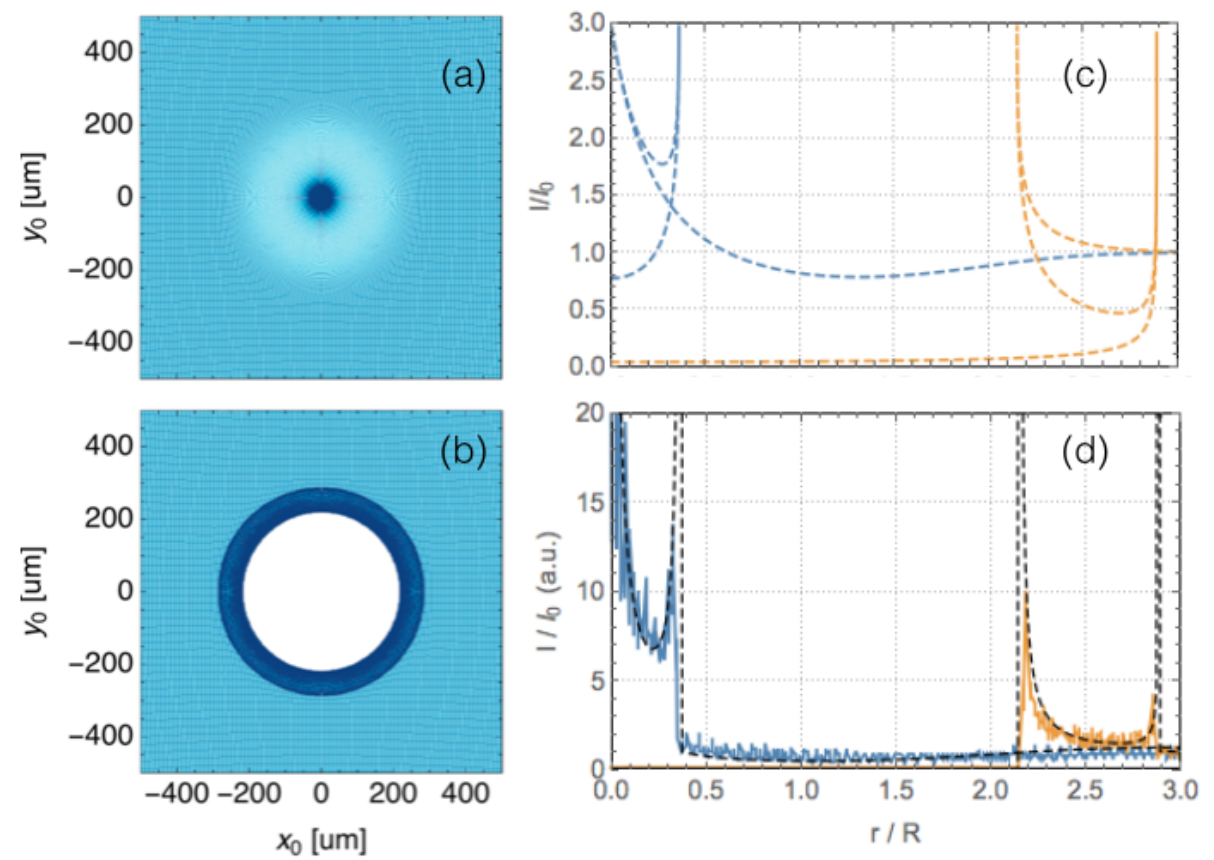

Figure 6.3 : Comparison between theory and simulation results in the caustic regime. In these simulations a monoenergetic $\mathcal{E}_{p}=14.7 \mathrm{MeV}$ proton source of $\left|z_{s}\right|=1 \mathrm{~cm}$, $z_{i}=10 \mathrm{~cm}$ interacts with a single magnetic filament of the form given by equation (6.3) having $a=100 \mu \mathrm{m}$ and $b=300 \mu \mathrm{m}$. (a-b) show simulated radiograph results using $B_{0}=2 \times B_{0 \text { crit }}$ for the focusing and defocusing cases, respectively. The color scale is fixed between images with darker (lighter) regions indicating a surplus (deficit) of protons. (c) shows the multi-branched caustic structures predicted by the parametric equations (6.14) and (6.15) using $\epsilon=0.5$. (d) shows normalized lineouts of the simulated proton fluence along $y_{0}=0$ with the blue curve corresponding to the focusing case and the yellow curve corresponding to the defocusing case. The complete analytic results formed by summing over all three branches of each curve in (c) are indicated by the dashed black lines in (d), exhibiting close agreement with the simulation results. 
malized image plane intensity is then given by,

$$
\frac{I}{I_{0}}=\frac{1+\epsilon}{\epsilon+\left|e^{-2 t^{2}}\left(\nu \mp e^{t^{2}}\right)\left(e^{t^{2}} \mp \nu\left(1-2 t^{2}\right)\right)\right|}
$$

in concert with equation $(6.14)$ for $r / R$. The transformation $z_{s} \rightarrow \tilde{z_{s}}$ where $\tilde{z}_{s}=$ $z_{s} z_{i} /\left(z_{i}-z_{s}\right)$ enhances the accuracy of equations (6.13-6.15) by relaxing constraints on the relationship between $z_{i}$ and $z_{s}[98]$.

We now validate the synthetic radiographs produced by the numerical tool through comparison to equations (6.13-6.15). Fig. 6.2 shows the results of this procedure for four simulations in the linear regime. (a-b) show synthetic proton radiographs generated by the tool using $B_{0}=0.2 \times B_{0 \text { crit }}$ for the focusing and defocusing cases, respectively. The color scale is fixed between images (and Figs. 6.2-6.6), with darker (lighter) regions indicating a surplus (deficit) of protons. The spatial coordinates are provided in nominal object plane units $x_{0}$ and $y_{0}$, i.e., $1 / M \times x, y$. (c) depicts normalized lineouts of the proton fluence along $y_{0}=0$ with the blue curve corresponding to the focusing simulation and the yellow curve corresponding to the defocusing simulation. The black dashed curves correspond to analytic theory from equation (6.14). (d-f) show the same set of plots for simulations and theory corresponding to the field strength $B_{0}=0.9 \times B_{0 c r i t}$. Panels (c) and (f) highlight the excellent agreement between theory and the simulated radiographs across conditions.

Fig. 6.3 shows results comparing simulations to the predictions of equations (6.14) and (6.15) for proton imaging in the nonlinear regime. (a-b) show the synthetic proton radiographs having nonlinear field strength $B_{0}=2 \times B_{0 \text { crit }}$ for the focusing and defocusing cases, respectively. (c) shows the multi-branched caustic structures predicted by the parametric equations (6.14) and (6.15) using $\epsilon=0.5$. (d) shows normalized lineouts of the simulated proton fluence along $y_{0}=0$ with the blue curve correspond- 
ing to the focusing case and the yellow curve corresponding to the defocusing case. The complete analytic results formed by summing over all three branches of each curve in (c) are indicated by the dashed black lines in (d). As we have a point source, the intensity experiences discontinuities on one side of the caustic. For $\epsilon=0$, analytically the caustic intensity tends towards infinity in this situation. Recent germane experimental results have suggested that $I / I_{0} \sim 3$ in practice[77], illustrating the importance of $\epsilon>0$ accounting for finite resolution effects.[98] Consistent with this finding (d) shows that the simulation output closely matches the analytics, bolstering confidence in its numerical fidelity.

\subsection{Application to the filamentation instability in millimeter- scale HED plasmas}

We have developed PRIME in connection to laboratory astrophysics experiments performed by the ACSEL collaboration[97, 180]. These experiments use powerful lasers to create high velocity plasmas flows by ablating the surface of plastic $\left(\mathrm{CH}_{2}\right)$ targets. In a typical experiment two such targets are set up opposing one another and illuminated with laser light to study properties of the colliding plasma plumes. For our puposes here the typical plasma parameters $[98,180,181,187]$ are $n_{e}=1 \times 10^{19} \mathrm{~cm}^{-3}, T_{e}=T_{i}=1 \mathrm{keV}, v_{\text {flow }}=8 \times 10^{7} \mathrm{~cm} / \mathrm{s}$. In the interaction between the two flows it is believed that the Weibel filamentation instability[209] plays an important role. Indeed, Weibel-like filamentary structures appearing in proton radiographs of the interaction have recently been reported $[77,56]$. Yet for the reasons described above the challenge to discern the fields from their radiograph, i.e., to determine the extent to which filamentary magnetic fields produce filamentary radiograph structures, persists. Realistic situations introduce further questions: will 


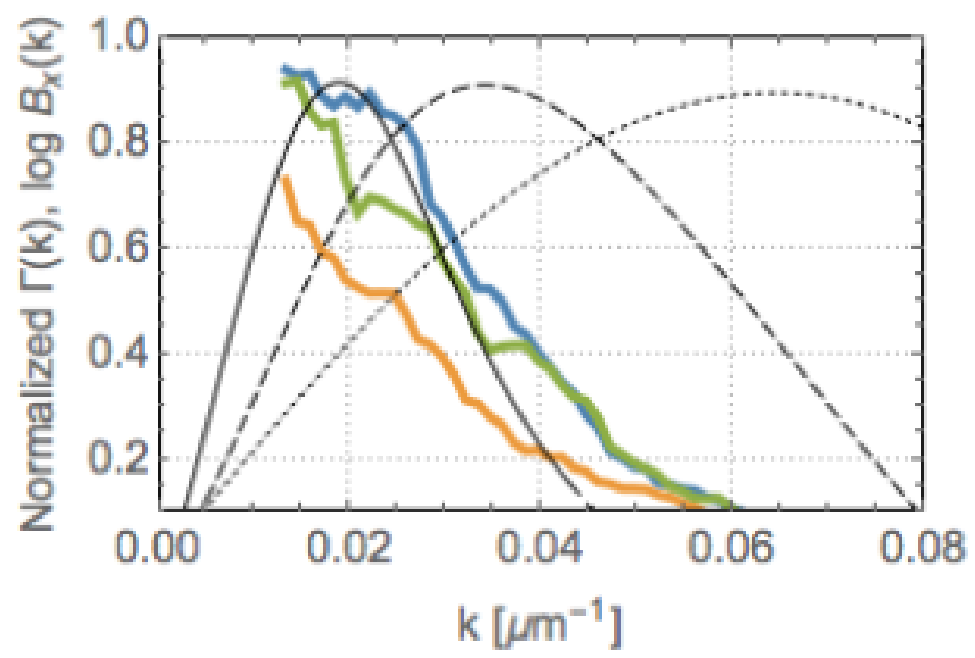

Figure 6.4: Comparison of simulation fields to the relevant transverse Weibel instability modes. The black curves correspond to the normalized instability growth rates $\Gamma(k)$ for collisionless Carbon (dotted), collisional Carbon (dashed) and collisional $\mathrm{CH}_{2}$ flows (solid) from equations (6.16-6.17). The colored curves correspond to normalized Fourier transformations $\log B_{x}(k)$ across $\hat{x}$ at the simulation midplane for three simulations of 'forests' of magnetic filaments (see text).

protons traversing the hundreds of magnetic filaments expected in a realistic situation produce a coherent radiograph, or will they scatter; how important are density and temperature heterogeneities expected in the plasma flows; what is role of field strength as the filaments grow over time; and ultimately if a coherent radiograph can be produced how does its periodicity correspond to that of the underlying fields. Resolving these complications will evidently require many simulations, and due to the plasma's $\sim 10 \mathrm{~mm}^{3}$ scale computational expense implies that multidimensional hydrodynamic and PIC simulations will not be ideally suited to this purpose. Our purpose here is to show that, using electromagnetic primitives to construct representative filamentary fields, PRIME simulations can provide insight into this situation. To this end we address a subset of these questions in this section. 
We construct a representative field topology, guided by the reported experimental conditions[77], using many dozens of magnetostatic Gaussian 'cocoons' of the form given in equation (6.3). The experimental results imply that filaments form within a $\sim 1.5 \mathrm{~mm}$ radius cylinder in the interaction midplane, with axial coordinate directed between the opposing plastic targets. We model this as a 'forest' of 260 filaments each instantiated with a random centroid position in the $x_{0}-z_{0}$ plane (at $y_{0}=0$, oriented along $\hat{y})$ within $x_{0}^{2}+z_{0}^{2} \leq(1.5 \mathrm{~mm})^{2}$. Experimental conditions also imply that $c / \omega_{p i}=100 \mu m$ and the axial length of the cylinder containing the filaments $\sim 0.5 \mathrm{~mm}$, so in the simulation each filament has $a=50 \mu m, b=500 \mu m$, meaning that the inverse wavenumber of the filament centroids is nominally $2 a=c / \omega_{p i}$. We further use randomized tilt parameters $\delta \theta, \delta \psi=15^{\circ}$ to account for natural density perturbations occurring in the plasma. Since these perturbations affecting the filament growth can be expected to vary between experiments, and since we are interested in determining whether filamentary structures in the radiographs are a robust signature of filamentary magnetic fields, we instantiate this setup in three distinct simulations. That is, we perform three simulations pursuant to these conditions, meaning that the filament centroid positions in $x_{0}-z_{0}$ and the individualized tilts of filaments will vary between simulations, while each filament $a$ and $b$ and the density of filaments $\left(\sim 75 / \mathrm{mm}^{3}\right)$ across simulations are constant.

To see that these simulation conditions form a reasonable approximation of experimental conditions, it is instructive to consider the relevant Weibel instability growth rates. For the purely transverse mode the collisionless dispersion relation is given by,

$$
k^{2}+\frac{\Gamma \sigma}{|k| U_{e}+\Gamma}+\frac{\Gamma}{|k| U_{i}+\Gamma}=\frac{k^{2}}{\Gamma^{2}+\frac{3 k^{2} S}{5}}
$$




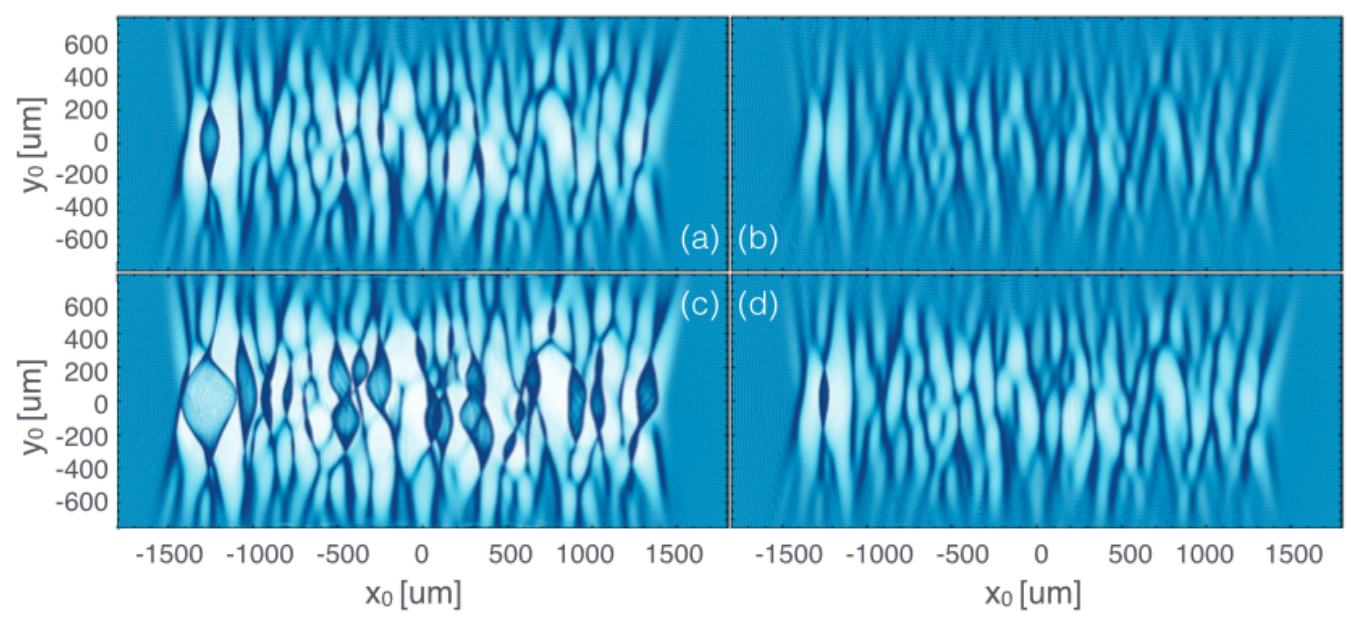

Figure 6.5 : Effects of field strength $B_{0}$ and probing proton energy $\mathcal{E}_{p}$ on the simulated proton radiograph results. All panels here correspond to the sim. 99 configuration indicated by the green curve in Fig. 6.4. (a-b) correspond to probing proton energies of $\mathcal{E}_{p}=14.7 \mathrm{MeV}$ and (c-d) to $\mathcal{E}_{p}=3 \mathrm{MeV}$. (a) and (c) correspond to $B_{0}=1 M G$ (meaning a peak simulation field of $0.4 M G$ ), and (b) and (d) correspond to $B_{0}=$ $0.3 M G$.

where $\Gamma$ is the growth rate normalized to $v_{\text {flow }} \omega_{p i} / c, k$ is the wave number normalized to $\omega_{p i} / c, U_{e, i}=v_{T e, i} /\left(\sqrt{\pi} v_{\text {flow }}\right)$ for thermal velocity $v_{T}, S=0.014 T_{i}[\mathrm{keV}]$ and $\sigma=A m_{p} /\left(Z m_{e}\right)$ for atomic mass $A$ and charge state $Z .[10,187]$ The dispersion relation accounting for collisional effects[187] can be formulated as,

$$
\begin{array}{r}
k^{2}+\frac{\Gamma}{\Gamma+k^{2} V_{s}}+\frac{\sigma\left(\Gamma+k^{2} R\right)}{\Gamma+k^{2} V_{\mathrm{se}}}= \\
\frac{k^{2}}{\Gamma\left(k^{2} V_{b}+\Gamma\right)+k^{2} S}
\end{array}
$$

where $R=0.00106 /\left(T_{e}[\mathrm{keV}]\right)^{3 / 2}, V_{s}=0.0175\left(T_{i}[\mathrm{keV}]\right)^{5 / 2}, V_{b}=0.0253\left(T_{i}[\mathrm{keV}]\right)^{5 / 2}, V_{\mathrm{se}}=$ $64\left(T_{e}[\mathrm{keV}]\right)^{5 / 2}$.

Equations (6.16-6.17) provide physical references for filament periodicity in the simulations. Fig. 6.4 shows the simulation fields in relation to the normalized $\Gamma$ curves 
for collisionless Carbon flows (dotted black), collisional Carbon flows (dashed black) and collisional $\mathrm{CH}_{2}$ flows (solid black) in which the light ions exhibit a stabilizing effect on the instability growth. The $\Gamma$ calculations assume plasma states of full ionization consistent with typical conditions that $T_{e}=T_{i}=1 \mathrm{keV}, v_{\text {flow }}=8 \times 10^{7} \mathrm{~cm} / \mathrm{s}$, and their depictions in Fig. 6.4 indicate the transverse Weibel modes which can be expected to grow most rapidly in the plasma. Since $\hat{z}$ is the axis of proton propagation the protons will deflect most strongly from the filamentary $B_{x}$ fields. The colored curves correspond to normalized Fourier transformations $\log B_{x}(k)$ across $\hat{x}$ at the simulation midplane for the three simulations: sim. 1 (blue), sim. 45 (orange) and sim. 99 (green). From Fig. 6.4 it is clear that the simulations provide an imperfect but reasonable approximation of the $k$-vectors which can be expected in the experimental situation.

Having described the simulation setup we now analyze the synthetic proton radiographs generated by PRIME for these cases. First we consider the roles of the magnetic field strength and proton beam energy for a single field configuration. Fig. 6.5 shows the simulated proton radiographs for two values of $B_{0}$ and two values of $\mathcal{E}_{p}$. (a) corresponding to the $B_{0}=1 M G\left(B_{\text {peak }} \simeq 0.4 M G\right)$ field strength and $\mathcal{E}_{p}=14.7$ $\mathrm{MeV}$ proton source closely approximates the calculated field values and the experimental conditions reported on in ref. [77]. In this simulated radiograph we observe coherent, predominantly vertical filamentary features striated along the plasma flow axis $(\hat{y})$. This fact is striking since according to ref. [98] protons should deflect in a nonlinear fashion from each of several dozen filamentary field structures on their path to the detector. Through examination of $(b-d)$ it is clear that these filamentary features persist across a variety of configurations. Comparison of (a) and (b) further shows that a reduction in field strength causes an apparent contraction of the plasma flow interaction region. The potential conflation in this regard forms an important 


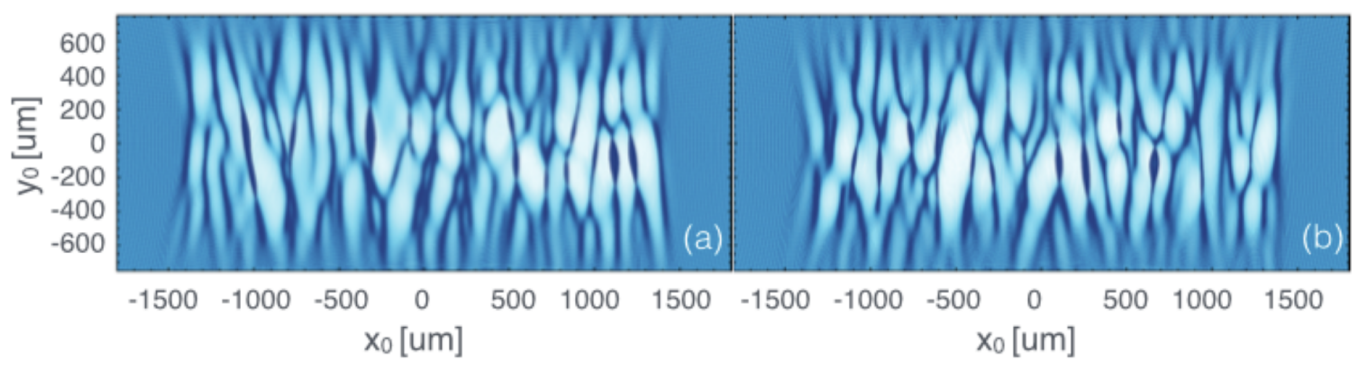

Figure 6.6 : Synthetic proton radiographs for (a) sim. 18 and (b) sim. 45. Across simulations $B_{0}=1 M G$ and $\mathcal{E}_{p}=14.7 \mathrm{MeV}$. The radiograph corresponding to these conditions for sim. 99 is shown in Fig. 6.5 (a).

consideration for experimental diagnosis.

To examine the robustness of filamentary radiograph structures we examine the sim. 18 and sim. 45 field configurations. Fig. 6.6 depicts these images, which are seen to clearly exhibit similar coherent, predominantly vertical filamentary features. In order to characterize the relationship between the field periodicity and the radiograph periodicity we have analyzed lineouts of the proton fluence along $y_{0}=0$ for each of the simulations. Fig. 6.7 (a) shows the magnitude of the Fourier-transformed periodicity from each radiograph. In (b-d) these radiograph periodicities (solid lines) are compared to the underlying magnetic field periodicities (dashed lines). From these figures it is clear that the radiograph signal is shifted to much shorter wavelengths than those found in the simulation. Furthermore the radiograph signal is negligible at the low $k$-values which dominate the magnetic field spectra. These results show that, at minimum for the cases considered here, filamentary structures in proton radiographs are a qualitative signature of Weibel instability-like filamentary magnetic fields.

We increment our systematic study of magnetic filament systems by considering 

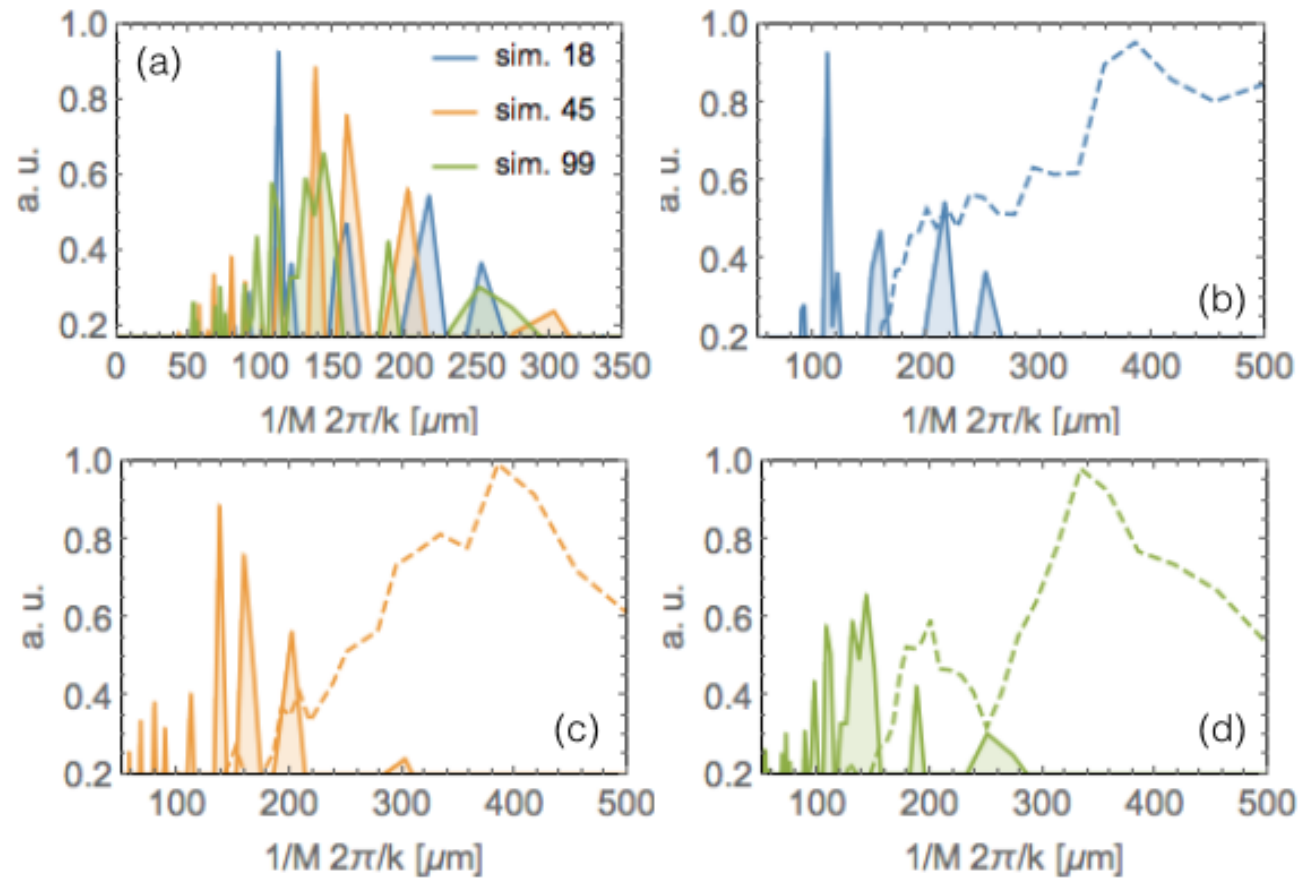

Figure 6.7 : Comparison between field periodicity and proton radiograph image periodicity. Solid lines correspond to Fourier-transformed lineouts at $y_{0}=0$ of the synthetic radiographs shown in Fig. 6.6. The dashed curves in (b-d) correspond to $2 \pi / k$ for the simulation $k$-vectors shown in Fig. 6.4. 


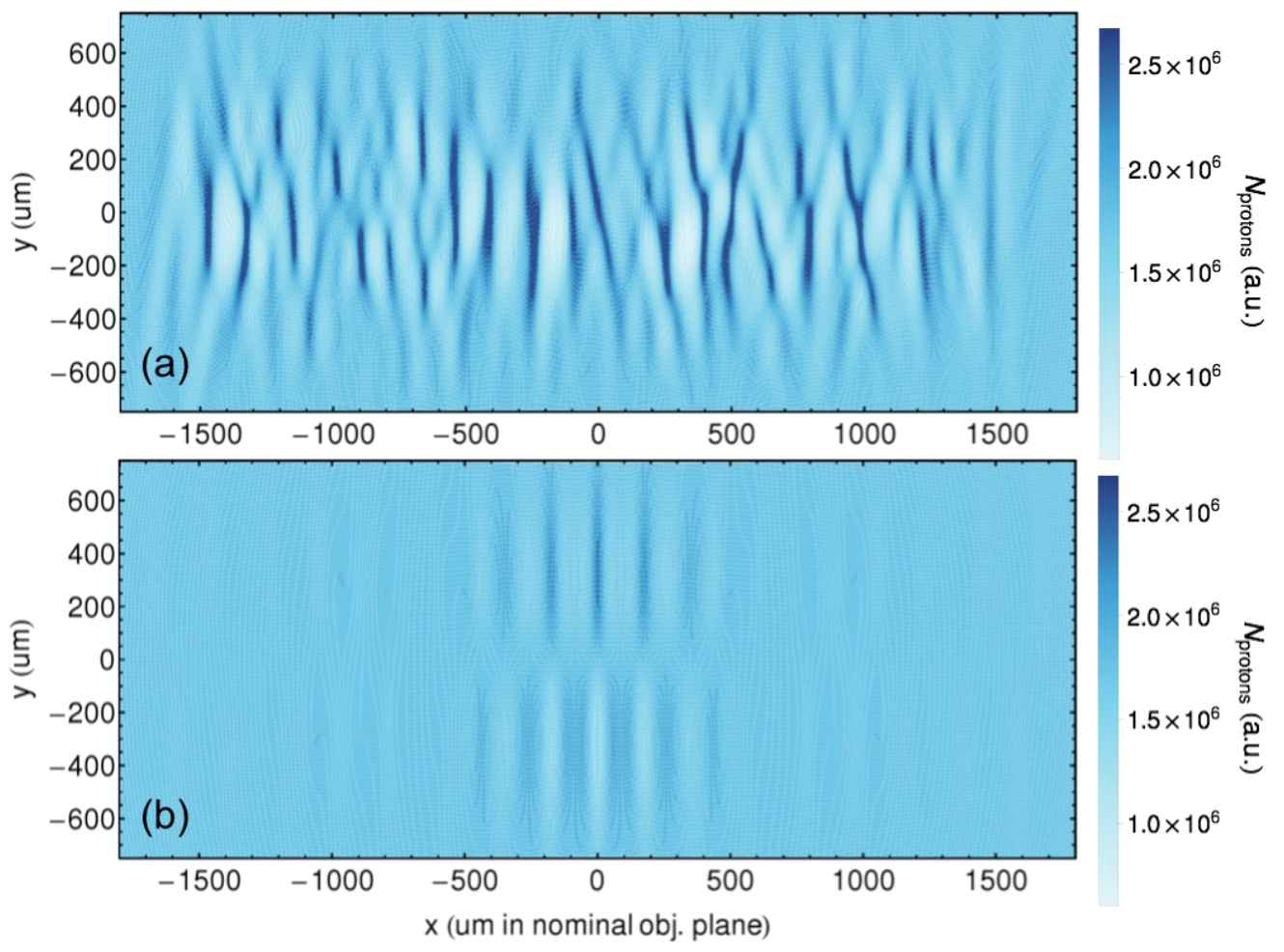

Figure 6.8 : Role of filament tilting and of the simulation field $k$ vector function on the resulting proton radiograph signal. (a-b) show synthetic radiographs generated by PRIME using the field control vector $\vec{L}=$ $(4,16,16,200 \mu \mathrm{m}, 200 \mu \mathrm{m}, 50 \mu \mathrm{m}, 500 \mu \mathrm{m}, 0,0,0.3 \mathrm{MG})$, and the same proton source and detector as in Figs. 6.4 - 6.7. (a) corresponds to the application of individualized tilt parameters $\delta \psi, \delta \theta=15^{\circ}$ while (b) uses $\delta \psi, \delta \theta=0$. 
a situation having a single, well defined $k$-vector. Specifying the field control vector in equation (6.2) to be $\vec{L}=(4,16,16,200 \mu \mathrm{m}, 200 \mu \mathrm{m}, 50 \mu \mathrm{m}, 500 \mu \mathrm{m}, 0,0,0.3 \mathrm{MG})$, we create a body-centered rectangular prism lattice of 256 magnetic filaments. Hence, in this simulation $k^{-1}=200 \mu \mathrm{m}$ with filament density $\sim 50 \mathrm{~mm}^{-3}$, compared to $100 \mu \mathrm{m}$ and $75 \mathrm{~mm}^{-3}$, respectively, for randomized simulations covered by Figs. 6.4 - 6.7. Fig. 6.8 shows the synthetic radiographs generated by PRIME for this system for both $\delta \psi, \delta \theta=15^{\circ}$ in (a) and $0^{\circ}$ in (b), using the same $14.7 \mathrm{MeV}$ point proton source and detector properties as above. In (a) we observe filamentary features elongated along the plasma flow axis exhibiting substantial qualitative similarities to the radiographs shown in Figs. 6.5 - 6.6. (b) shows that the tilting of the field filaments, a feature expected in realistic situations, plays an important role in the simulated radiograph signal. In this panel we observe that both the amplitude and quantity of the structures present in the radiograph are reduced. Fig. 6.9 shows the Fourier transformations of the normalized lineouts of proton fluence along $y_{0}=0$ for these radiographs. For the $\delta \psi, \delta \theta=0$ simulation the signal in the midplane is zero, due to perfect cancellation of deflections to the proton trajectories passing through each symmetric magnetic filament, consistent with the calculations of Kugland et al.[98]. $y_{0}=0$ also forms the axis of symmetry of the probing proton transverse velocity. Consistent with the fact that $v_{y}$ reverses sign at this point, we observe that protons 'focus' in the radiograph for $y_{0}>0$ and 'defocus' below $y_{0}<0$. It is anticipated that this intuitive, significant aspect will form an important consideration for future work analyzing the quantitative relationship between fields and their radiograph in more realistic situations, e.g., as shown in Fig. 6.8 (a). We note that the periodicity of the proton fluence in 6.8 (b) above or below $y_{0}=0$ is approximately $200 \mu \mathrm{m}$, consistent with the simulation $k^{-1}$. Meanwhile, we observe that the periodicity for the tilted filament case shown in Fig. 6.9 is quite similar to the results shown in Fig. 6.7 (a). This similarity, despite the 


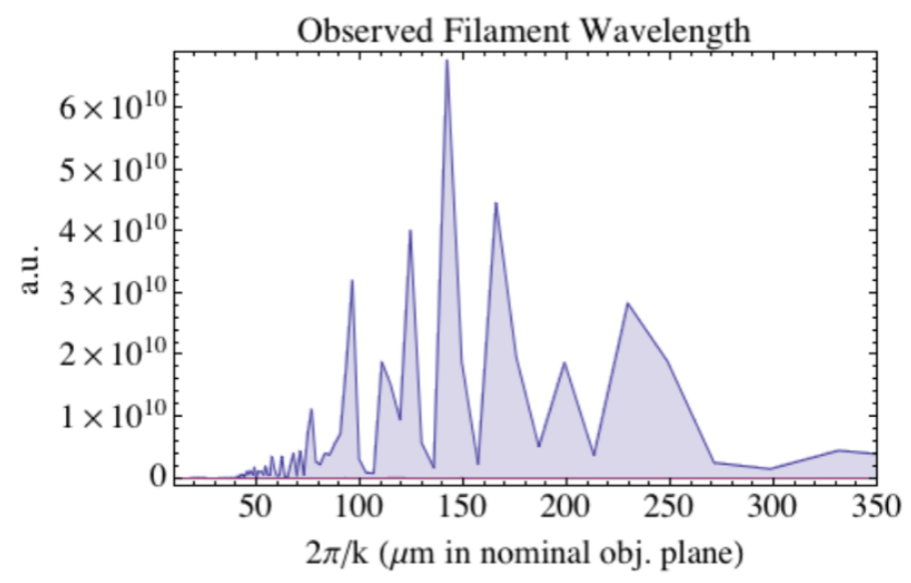

Figure 6.9 : Fourier transformations of the normalized lineouts of proton fluence along $y_{0}=0$ for the radiographs depicted in Fig. 6.8. The purple curve corresponds to the $\delta \psi, \delta \theta=15^{\circ}$ case while enhanced signal in the midplane is absent for the $0^{\circ}$ case.

disparate $k^{-1}$ and filament densities between simulations, suggests the continuing role of prudence in assessing more realistic situations.

To this point we have considered the geometry in which probing protons sample the fields normal to the axis of the plasma flow. This geometry is motivated by the standard experimental geometry of the ACSEL group.[97, 96, 180, 181] We now ask the question: in future experiments what kinds of radiograph structures could we expect for proton sampling along the flow axis?

With the aim of guiding future experiments we have simulated this situation using PRIME ; Fig. 6.10 shows the results of the sim. 99 field geometry imaged along the flow axis, i.e., along the major axis of the filaments, using a proton source created by the source control vector $\vec{S}=\left(2,14.7 \mathrm{MeV}, 10^{9}, 1 \mathrm{~cm}\right)$. (a) corresponds to $B_{0}=0.3 \mathrm{MG}$ and $(\mathrm{b})$ corresponds to $B_{0}=1 \mathrm{MG}$. These simulated radiographs show that the filamentation instability imaged along the flow axis exhibits distinct 


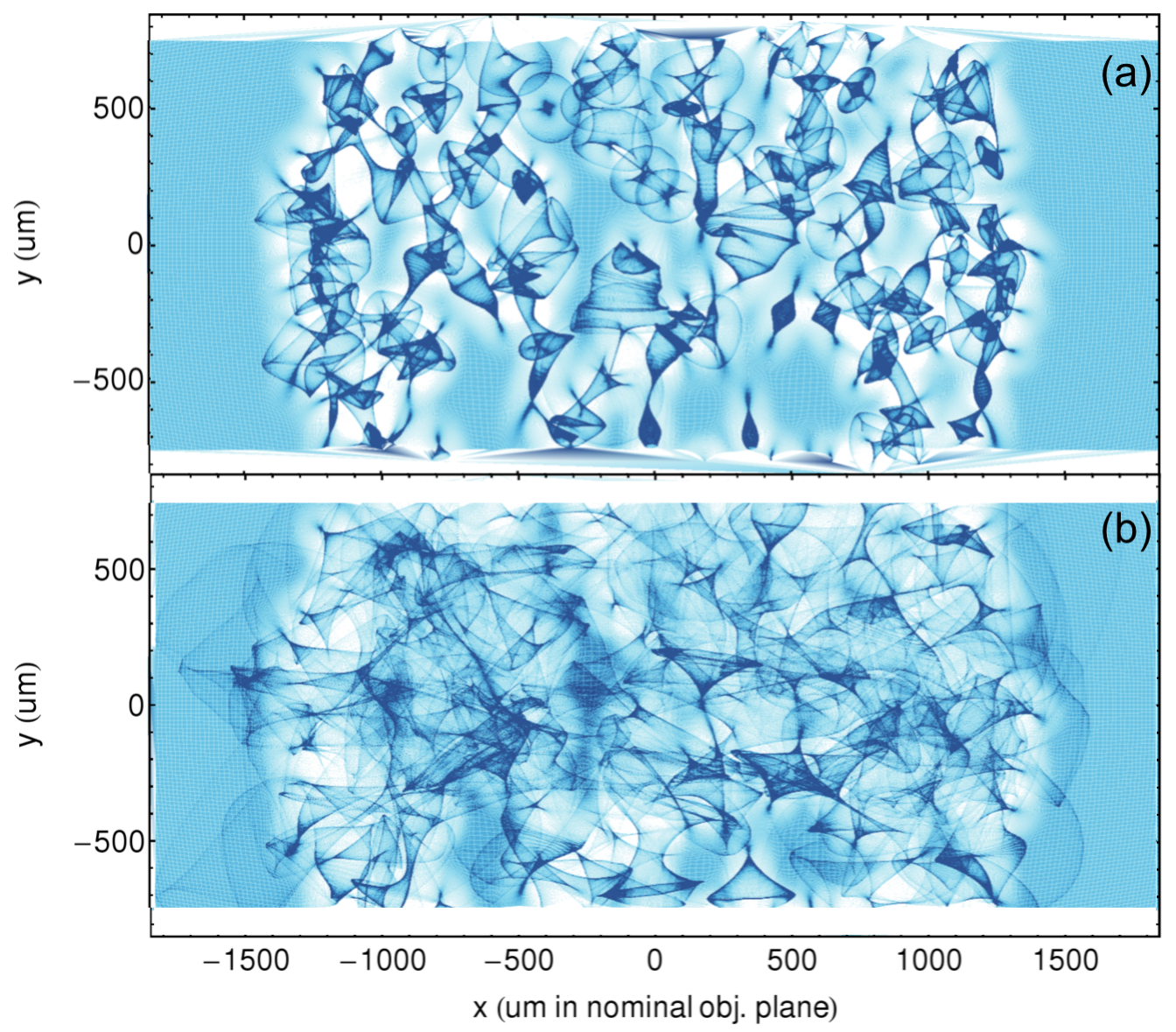

Figure 6.10 : Proton radiographs of the the sim. 99 field geometry imaged along the axis of the plasma flows. The $14.7 \mathrm{MeV}$ proton source is otherwise identical to that used for Figs. 6.5 - 6.8. (a) corresponds to $B_{0}=0.3 \mathrm{MG}$ and (b) corresponds to $B_{0}=1 \mathrm{MG}$. 
and beautiful structure. Since this geometry is inherently more two dimensional, it is anticipated that imaging in this manner could provide unique insights into the field spatial structures and transverse profiles. Deflections in the caustic regime are observed in (a), at lower field strength than in the 'flow-normal' geometry. This is consistent with the fact that the integrated field sampled by a given proton is associated with the filament major axis $b$ rather than semi-major axis $a$ (with $b>a$ ). By equation (6.3), protons propagating down the major axis of a given filament experience a focusing force leading to a proton surplus in the resulting radiograph. In (b) we observe that the field strengths are sufficient to cause the protons to interact in a predominantly scattering mode. Yet even in this case essential, direct information about the field's transverse spatial extent is supplied by imaging in this geometry.

To develop understanding of the relationship between the radiograph periodicity and the underlying field periodicity in the 'flow-along' geometry, we have generated synthetic radiographs of the lattice situation described by Fig. 6.9. The simulated radiographs for this situation are shown in Fig. 6.11, with (a) corresponding to $\delta \psi, \delta \theta=0$ and (b) corresponding to $\delta \psi, \delta \theta=15^{\circ}$. In stark contrast to the flownormal geometry, these images show that the lattice structure of the underlying fields is to a large degree preserved in the radiograph images. This is so even in (b) accounting for realistic filament tilting effects. These simulation results hence illuminate a new path for experimental efforts seeking to understand and characterize the Weibel instability in counterstreaming plasma flows. We note that the apparent effect of the lattice 'expanding' near the center of the radiograph shown in (a) is illusory: the signal associated with each filament in this region exhibits a multi-branched caustic structure that extends over a larger region. The protons interacting with filaments away from the central region have a larger transverse velocity and thus sample the 

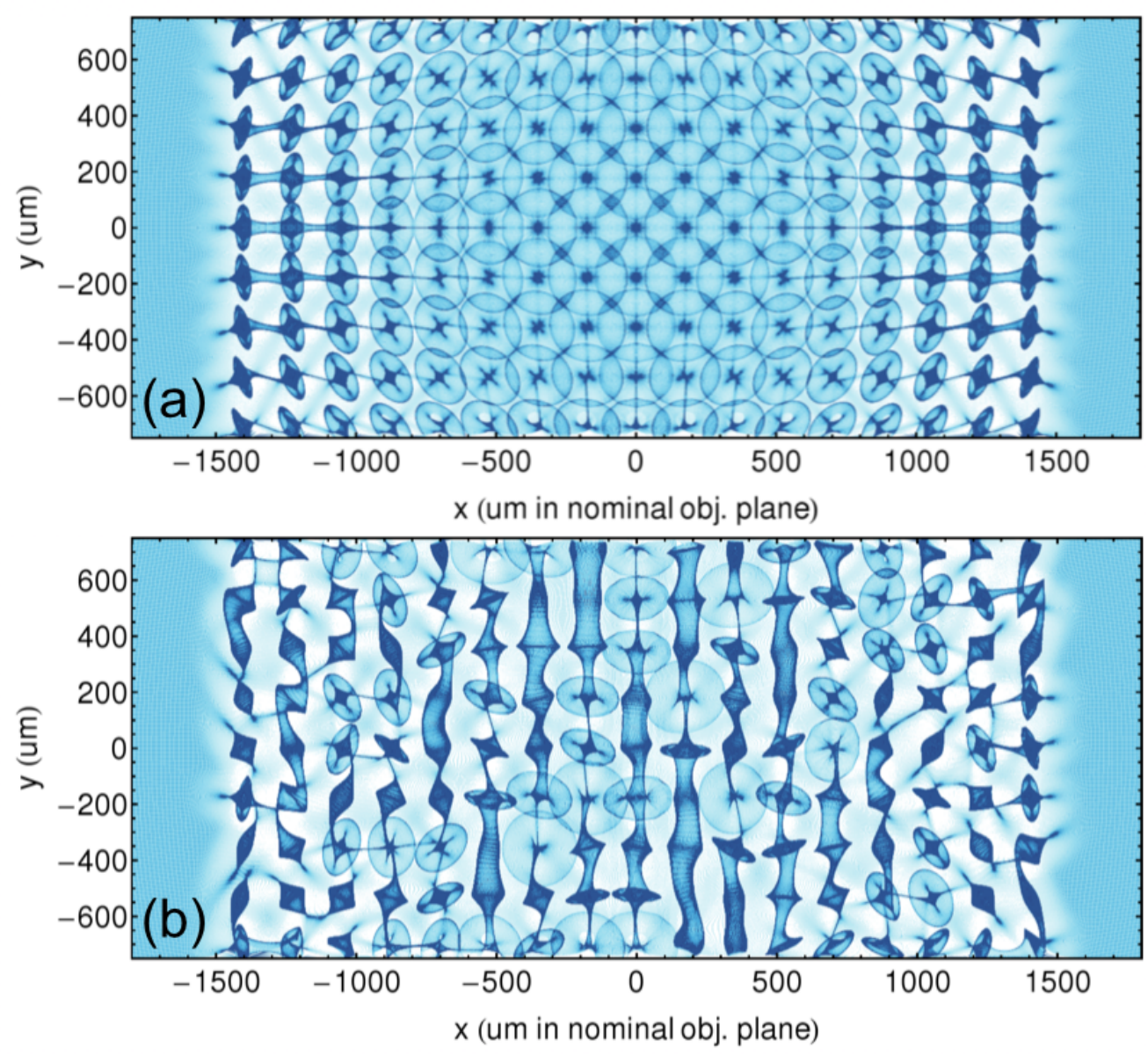

Figure 6.11 : Proton radiography imaging along the plasma flow axis. Apart from the proton axis of propagation the simulation field and proton source properties are identical to those covered in Fig. 6.8. (a) corresponds to $\delta \psi, \delta \theta=0$ while (b) uses $\delta \psi, \delta \theta=15^{\circ}$. 
field of a single filament over a shorter distance. The resulting focusing force is accordingly lower, meaning that the multi-branching caustic regime is not reached. In (b) we observe that the filament tilting has the effect of causing signal elongation in $\hat{y}$. Meanwhile the the periodicity in $\hat{x}$ is largely preserved; in both (a) and (b) we calculate that the radiograph $k^{-1}$ is within $10 \%$ of the underlying field inverse wavenumber. While future work will provide a detailed analysis of these situations, it seems plausible that this geometry offers a promising new opportunity for coming experimental campaigns. The ability to diagnose the field structure and amplitude may serve as a stepping stone to future identification of exotic QED processes such as magnetic pair creation and resonant Compton scattering in the high-field Schwinger domain[194]. This offers a connection to prospects realizable for fundamental physics when more intense lasers technology becomes available.

In this chapter we have presented a new simulation tool for interpreting proton radiography of HED plasmas. The present tool's numerical approach captures all relevant physics effects, including effects related to the formation of caustics. Electromagnetic fields can be imported from PIC or hydrodynamic codes in a streamlined fashion. A library of electromagnetic field 'primitives' is also provided. These primitives can be considered 'eigenvectors,' in effect spanning the basis of electromagnetic fields, such that through linear combinations the user may construct realistic field topologies by hand. This capability allows users to add a primitive, modify the field strength, rotate a primitive, and so on, while quickly generating a high resolution radiograph at each step. In this way, PRIME enables the user to deconstruct features in a radiograph and interpret them in connection to specific underlying electromagnetic field elements. We have applied the tool in connection to experimental observations of the Weibel instability in counterstreaming plasmas, using $\sim 10^{8}$ particles generated from a realistic laser-driven point-like proton source, imaging fields which cover 
volumes of $\sim 10 \mathrm{~mm}^{3}$. Imaging normal to the plasma flow axis, insights derived from this application indicate that tilting of magnetic filaments plays a significant role in setting the proton image; field strength tends to affect the apparent axial lengthscale over which the filamentation instability is active; and coherent imaging is possible in the sense that filamentary structures are observed in radiographs as a signature of the Weibel fields, at least for the cases considered here. Imaging along the plasma flow axis, we have shown that the filamentation instability exhibits a beautiful lattice-like signature. This signature is further useful: it provides a clear, more two dimensional picture of Weibel-driven filamentary spatial structures and $k$-vectors, as well as the transverse spatial profiles of the plasmas flows. This utility illuminated by PRIME simulations should guide future experimental efforts. Future research will focus on parsing the quantitative relationship between the field and radiograph periodicities, a task which exceeds the scope of this chapter. These results show that PRIME can support understanding of HED plasmas. 


\section{Chapter 7}

\section{Conclusions \& future research}

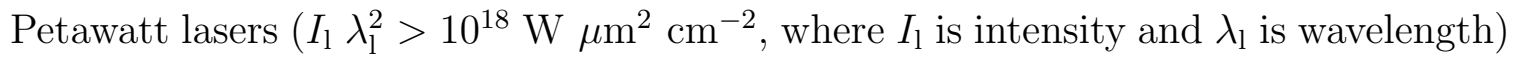
are the most powerful light sources ever created on earth. Illuminating solids by their light creates extreme states of matter with temperatures exceeding ten million degrees Celsius and pressures exceeding one billion earth atmospheres. These high energy density (HED) conditions are driven at the microscopic scale by dense currents of relativistic electrons $\left(\sim 10^{11} \mathrm{~A} \mathrm{~cm}^{-2}\right)$, oscillating violently in the intense laser fields $\left(>10^{10} \mathrm{~V} \mathrm{~cm}^{-1}\right)$, as well as the plasma processes arising when these particles are dephased and injected into the high density target.In principle this setup opens the way to advanced scientific applications such as compact relativistic particle accelerators, laser fusion, laboratory astrophysics, ultrafast imaging systems, high-energy radiation sources and intense high harmonic generation. In practice realizing this potential coincides with developing models of absorption that elucidate and ultimately facilitate control of mechanisms by which laser energy is converted to target particle energy. Indeed, key metrics for petawatt laser applications relate to absorption, yet the germane HED conditions are so nonlinear that it is often impossible to know the fraction of absorbed light $f$, and even the range of $f$ is unknown. In this thesis theoretical models of the absorption of high power laser light by matter have been derived (chapters 3-4), applications of these models have been investigated (sections 3.5 - 3.6 ), and simulation tools supporting the diagnosis and implementation of these applications have been developed (chapter 6). In particular, chapter 3 developed 
an advanced relativistically-correct theoretical model of petawatt laser absorption by optically-thick targets, accounting for both ion and electron beam aspects of the interaction. Predictions of the model for the energetic properties of these beams, as well as the dynamical motion of the laser-matter interface, have been elucidated in section 3.1. Results from high resolution, relativistic, kinetic particle-in-cell simulations using the LSP code were shown to be in good agreement with the model in section 3.1.6. The theoretical maximum and minimum absorption values in laser-solid interactions were derived from the model in a general fashion in section 3.5. These bounds constrain nonlinear absorption mechanisms across the petawatt regime, forbidding high absorption values at low laser power and low absorption values at high laser power. For applications needing to circumvent the absorption bounds, these results will accelerate a shift from solid targets, towards structured and multilayer targets, and lead the development of new materials. These bounds further become increasingly strict with laser power, forbidding $35 \%$ of possible absorption values at $I_{1} \lambda_{1}^{2} \sim 10^{22} \mathrm{~W} \mu \mathrm{m}^{2} \mathrm{~cm}^{-2}$, a regime accessible at laser facilities such as ELI scheduled to come online in the next few years. These results will therefore play a central role in guiding the next generation of multi-petawatt experiments. In chapter 4 these results were extended to include effects related to heterogeneous plasmas, including relativistically-underdense plasmas relevant to 'pre-plasma' situations, and realistic laser spatio-temporal profiles.

Considerations of absorption processes at the 10-petawatt scale were developed, and a mechanism of focusing high power laser light to higher intensities, in a fashion that could support reaching the QED-plasma regime, was elucidated in chapter 5. Supporting the measurement and validation of these models, the development of a new simulation tool for understanding high energy density plasmas, based on the proton radiography technique, was detailed in chapter 6 . The collective effect of 
these works is to advance the understanding of absorption, the conversion of light energy $\hbar \omega$ to matter energy $\gamma m c^{2}$, in the context of the most powerful light sources realized by human science and technology.

Future work will apply the proton radiography tool to understanding experimental results measured by the ACSEL collaboration related to Weibel-mediated collisionless shock formation in high Mach number counterstreaming plasma flows. The ability to diagnose the field structure and amplitude may serve as a stepping stone to future identification of exotic QED processes such as magnetic pair creation and resonant Compton scattering in the high-field Schwinger domain. This offers a connection to prospects realizable for fundamental physics when more intense lasers technology becomes available. The petawatt-scale absorption models developed in this thesis further form foundations for addressing topical problems relevant to the high power laser physics and high field physics communities. The focus of future research naturally falls on extending these models to the sub-petawatt and 10-petawatt laser power regimes. Extensions to the sub-petawatt regime, in which laser absorption processes are predominantly collisional, hold promise in connection to research on inertial confinement fusion topics. At the 10-petawatt scale, the possibility of laser absorption through electron-positron pair creation channels, not accounted for in the present model, is realized. Hence research programs beginning in the near term will focus on extending the absorption model to treat pair production processes, with the objective of spanning the intensity interval from present laser technology, $\sim 10^{22} \mathrm{~W} \mu \mathrm{m}^{2} \mathrm{~cm}^{-2}$, to the Schwinger limit, $\sim 10^{29} \mathrm{~W} \mu \mathrm{m}^{2} \mathrm{~cm}^{-2}$. The development of absorption models covering this range promises a fundamental description of the interaction of light and matter under the most extreme conditions present in the universe. 


\section{References}

[1] ELI - extreme light infrastructure, 2014.

[2] Lawrence Livermore National Laboratory: Inertial Confinement Fusion, 2014.

[3] Alexey V. Arefiev, Boris N. Breizman, Marius Schollmeier, and Vladimir N. Khudik, Parametric Amplification of Laser-Driven Electron Acceleration in Underdense Plasma, Physical Review Letters 108 (2012), no. 14, 145004.

[4] M. G. Baring, Temporal evolution of pair attenuation signatures in gamma-ray burst spectra, Astrophys. J. 650 (2006), no. 1004.

[5] Teresa Bartal, Mark E. Foord, Claudio Bellei, Michael H. Key, Kirk a. Flippo, Sandrine a. Gaillard, Dustin T. Offermann, Pravesh K. Patel, Leonard C. Jarrott, Drew P. Higginson, Markus Roth, Anke Otten, Dominik Kraus, Richard B. Stephens, Harry S. McLean, Emilio M. Giraldez, Mingsheng S. Wei, Donald C. Gautier, and Farhat N. Beg, Focusing of short-pulse high-intensity laseraccelerated proton beams, Nature Physics 8 (2011), no. 2, 139-142.

[6] F. N. Beg, a. R. Bell, a. E. Dangor, C. N. Danson, a. P. Fews, M. E. Glinsky, B. a. Hammel, P. Lee, P. a. Norreys, and M. Tatarakis, A study of picosecond lasersolid interactions up to 1019 Wcm2, Physics of Plasmas 4 (1997), no. 2, 447.

[7] A. Bell and John Kirk, Possibility of Prolific Pair Production with High-Power Lasers, Physical Review Letters 101 (2008), no. 20, 200403. 
[8] A. R. Bell and R. J. Kingham, Resistive Collimation of Electron Beams in Laser-Produced Plasmas, Physical Review Letters 91 (2003), no. 3, 035003.

[9] C. Bellei, L. Divol, A. J. Kemp, M. H. Key, D. J. Larson, D. J. Strozzi, M. M. Marinak, M. Tabak, and P. K. Patel, Fast ignition: Dependence of the ignition energy on source and target parameters for particle-in-cell-modelled energy and angular distributions of the fast electrons, Physics of Plasmas 20 (2013), no. 5, 052704 .

[10] R. L. Berger, J. R. Albritton, C. J. Randall, E. a. Williams, W. L. Kruer, a. B. Langdon, and C. J. Hanna, Stopping and thermalization of interpenetrating plasma streams, Physics of Fluids B: Plasma Physics 3 (1991), no. 1, 3-12.

[11] C. Birdsall and A. B. Langdon, Hilger, London, 1991.

[12] Charles K Birdsall and A Bruce Langdon, Plasma physics via computer simulation, CRC Press, 2004.

[13] J. C. McKinney \& R. D. Blandford, Stability of relativistic jets from rotating, accreting black holes via fully three-dimensional magnetohydrodynamic simulations, Mon. Notices R. Astron. Soc. 394 (2009), no. L126.

[14] Roger Blandford and David Eichler, Particle Acceleration at Astrophysical Shocks: A Theory of Cosmic Ray Origin, Physics Reports 154 (1987), no. 1, $1-75$.

[15] M. BORGHESI, P. AUdEBERT, S.V. BULANOV, T. COWAN, J. FUCHS, J.C. GAUThiER, A.J. MACKINNON, P.K. PATEL, G. PRETZLER, L. ROMAGNANI, A. SCHIAVI, T. TONCIAN, and O. WILLI, High-intensity laser- 
plasma interaction studies employing laser-driven proton probes, Laser and Particle Beams 23 (2005), no. 03, 291-295.

[16] M. Borghesi, D. H. Campbell, a. Schiavi, M. G. Haines, O. Willi, a. J. MacKinnon, P. Patel, L. a. Gizzi, M. Galimberti, R. J. Clarke, F. Pegoraro, H. Ruhl, and S. Bulanov, Electric field detection in laser-plasma interaction experiments via the proton imaging technique, Physics of Plasmas 9 (2002), no. 5, 2214.

[17] M. Borghesi, D.H. Campbell, a. Schiavi, O. Willi, a.J. Mackinnon, D. Hicks, P. Patel, L.a. Gizzi, M. Galimberti, and R.J. Clarke, Laser-produced protons and their application as a particle probe, Laser and Particle Beams 20 (2002), no. $02,269-275$.

[18] M Borghesi, J Fuchs, SV Bulanov, AJ Mackinnon, PK Patel, and M Roth, Fast ion generation by high-intensity laser irradiation of solid targets and applications, Fusion Science and Technology 49 (2006), no. 3, 412-439.

[19] M. Borghesi, S. Kar, L. Romagnani, T. Toncian, P. Antici, P. Audebert, E. Brambrink, F. Ceccherini, C.a. Cecchetti, J. Fuchs, M. Galimberti, L.a. Gizzi, T. Grismayer, T. Lyseikina, R. Jung, a. Macchi, P. Mora, J. Osterholtz, a. Schiavi, and O. Willi, Impulsive electric fields driven by high-intensity laser matter interactions, Laser and Particle Beams 25 (2007), no. 01, 161-167.

[20] M. Borghesi, L. Romagnani, A. Schiavi, D. H. Campbell, M. G. Haines, O. Willi, a. J. Mackinnon, M. Galimberti, L. Gizzi, R. J. Clarke, and S. Hawkes, Measurement of highly transient electrical charging following high-intensity lasersolid interaction, Applied Physics Letters 82 (2003), no. 10, 1529.

[21] M. Borghesi, G. Sarri, C.a. Cecchetti, I. Kourakis, D. Hoarty, R.M. Stevenson, S. James, C.D. Brown, P. Hobbs, J. Lockyear, J. Morton, O. Willi, R. Jung, 
and M. Dieckmann, Progress in proton radiography for diagnosis of ICF-relevant plasmas, Laser and Particle Beams 28 (2010), no. 02, 277-284.

[22] M Borghesi, A Schiavi, D H Campbell, M G Haines, O Willi, A J MacKinnon, L A Gizzi, M Galimberti, R J Clarke, and H Ruhl, Proton imaging: a diagnostic for inertial confinement fusion/fast ignitor studies, Plasma Physics and Controlled Fusion 43 (2001), no. 12A, A267-A276.

[23] Marco Borghesi, Carlo Alberto Cecchetti, Toma Toncian, Julien Fuchs, Lorenzo Romagnani, Satyabrata Kar, P A Wilson, Patrizio Antici, Patrick Audebert, Erik Brambrink, Ariane Pipahl, Munib Amin, Ralph Jung, Jens Osterholz, Oswald Willi, Wigen Nazarov, Robert J Clarke, Margaret Notley, David Neely, Patrick Mora, Thomas Grismayer, Guy Schurtz, Angelo Schiavi, Yasuhiko Sentoku, and Emanuel DHumieres, Laser-Driven Proton Beams: Acceleration Mechanism, Beam Optimization, and Radiographic Applications, IEEE Transactions on Plasma Science 36 (2008), no. 4, 1833-1842.

[24] F. Brunel, Not-So-Resonant, Resonant Absorption, Physical Review Letters 59 (1987).

[25] S.V Bulanov, T.Zh Esirkepov, V.S Khoroshkov, A.V Kuznetsov, and F Pegoraro, Oncological hadrontherapy with laser ion accelerators, Physics Letters A 299 (2002), no. 23, $240-247$.

[26] F Cattani, A Kim, D Anderson, and M Lisak, Threshold of induced transparency in the relativistic interaction of an electromagnetic wave with overdense plasmas, Physical review. E, Statistical physics, plasmas, fluids, and related interdisciplinary topics 62 (2000), no. 1 Pt B, 1234-7. 
[27] C. a. Cecchetti, M. Borghesi, J. Fuchs, G. Schurtz, S. Kar, a. Macchi, L. Romagnani, P. a. Wilson, P. Antici, R. Jung, J. Osterholtz, C. a. Pipahl, O. Willi, a. Schiavi, M. Notley, and D. Neely, Magnetic field measurements in laserproduced plasmas via proton deflectometry, Physics of Plasmas 16 (2009), no. 4, 043102.

[28] H. Chen, S. C. Wilks, James Bonlie, E. Liang, Jason Myatt, D. F. Price, D. D. Meyerhofer, and Peter Beiersdorfer, Relativistic Positron Creation Using Ultraintense Short Pulse Lasers, Physical Review Letters 102 (2009), no. 10, 1-4.

[29] H. Chen, S. C. Wilks, W. L. Kruer, P. K. Patel, and R. Shepherd, Hot electron energy distributions from ultraintense laser solid interactions, Physics of Plasmas 16 (2009), no. 2, 020705.

[30] H. Chen, S. C. Wilks, D. D. Meyerhofer, J. D. Bonlie, C. D. Chen, S. N. Chen, C. Courtois, L. N. Elberson, G. Gregori, W. L. Kruer, O. Landoas, J. Mithen, J. Myatt, C. Murphy, P. Nilson, D. F. Price, M. Schneider, R. Shepherd, C. Stoeckl, M. Tabak, R. Tommasini, and P. Beiersdorfer, Relativistic Quasimonoenergetic Positron Jets from Intense Laser-Solid Interactions, Physical Review Letters 105 (2010), no. 1, 1-4.

[31] Hui Chen, Scott Wilks, James Bonlie, Edison Liang, Jason Myatt, Dwight Price, David Meyerhofer, and Peter Beiersdorfer, Relativistic Positron Creation Using Ultraintense Short Pulse Lasers, Physical Review Letters 102 (2009), no. 10, $1-4$.

[32] S. N. Chen, E. DHumières, E. Lefebvre, L. Romagnani, T. Toncian, P. Antici, P. Audebert, E. Brambrink, C. a. Cecchetti, T. Kudyakov, a. Pipahl, Y. Sentoku, M. Borghesi, O. Willi, and J. Fuchs, Focusing Dynamics of High-Energy 
Density, Laser-Driven Ion Beams, Physical Review Letters 108 (2012), no. 5, 055001.

[33] George E. Collins, Quantifier elimination for real closed fields by cylindrical algebraic decomposition, Lecture Notes in Computer Science, vol. 33, Springer Berlin Heidelberg, 1975.

[34] Committee on High Energy Density Plasma Physics, Plasma Science Committee, and National Research Council, Frontiers in High Energy Density Physics: The X-Games of Contemporary Science, The National Academies Press, 2003.

[35] L. a. Cottrill, a. B. Langdon, B. F. Lasinski, S. M. Lund, K. Molvig, M. Tabak, R. P. J. Town, and E. a. Williams, Kinetic and collisional effects on the linear evolution of fast ignition relevant beam instabilities, Physics of Plasmas $\mathbf{1 5}$ (2008), no. 8, 082108.

[36] Hiroyuki Daido, Mamiko Nishiuchi, and Alexander S Pirozhkov, Review of laser-driven ion sources and their applications., Reports on progress in physics. Physical Society (Great Britain) 75 (2012), no. 5, 056401.

[37] J R Davies, Laser absorption by overdense plasmas in the relativistic regime, Plasma Physics and Controlled Fusion 51 (2009), no. 1, 014006.

[38] John Dawson, Particle simulation of plasmas, Reviews of Modern Physics 55 (1983), no. 2, 403-447.

[39] F. de Hoffmann and E. Teller, Magneto-Hydrodynamic Shocks, Physical Review 80 (1950), no. 4, 692-703.

[40] J. Denavit, Absorption of High-Intensity Subpicosecond Lasers on Solid Density Targets, Physical Review Letters 69 (1992), no. 21. 
[41] E. Dewald, J. Milovich, P. Michel, O. Landen, J. Kline, S. Glenn, O. Jones, D. Kalantar, a. Pak, H. Robey, G. Kyrala, L. Divol, L. Benedetti, J. Holder, K. Widmann, a. Moore, M. Schneider, T. Döppner, R. Tommasini, D. Bradley, P. Bell, B. Ehrlich, C. Thomas, M. Shaw, C. Widmayer, D. Callahan, N. Meezan, R. Town, a. Hamza, B. Dzenitis, a. Nikroo, K. Moreno, B. Van Wonterghem, a. Mackinnon, S. Glenzer, B. MacGowan, J. Kilkenny, M. Edwards, L. Atherton, and E. Moses, Early-Time Symmetry Tuning in the Presence of Cross-Beam Energy Transfer in ICF Experiments on the National Ignition Facility, Physical Review Letters 111 (2013), no. 23, 235001.

[42] T.R. Dittrich, O.a. Hurricane, D.a. Callahan, E.L. Dewald, T. Döppner, D.E. Hinkel, L.F. Berzak Hopkins, S. Le Pape, T. Ma, J.L. Milovich, J.C. Moreno, P.K. Patel, H.-S. Park, B.a. Remington, J.D. Salmonson, and J.L. Kline, Design of a High-Foot High-Adiabat ICF Capsule for the National Ignition Facility, Physical Review Letters 112 (2014), no. 5, 055002.

[43] R. P. Drake, High-energy-density physics: Fundamentals, inertial fusion, and experimental astrophysics, Springer, 2006.

[44] M. J. Edwards, J. D. Lindl, B. K. Spears, S. V. Weber, L. J. Atherton, D. L. Bleuel, D. K. Bradley, D. a. Callahan, C. J. Cerjan, D Clark, G. W. Collins, J. E. Fair, R. J. Fortner, S. H. Glenzer, S. W. Haan, B. a. Hammel, a. V. Hamza, S. P. Hatchett, N. Izumi, B. Jacoby, O. S. Jones, J. a. Koch, B. J. Kozioziemski, O. L. Landen, R. Lerche, B. J. MacGowan, a. J. MacKinnon, E. R. Mapoles, M. M. Marinak, M. Moran, E. I. Moses, D. H. Munro, D. H. Schneider, S. M. Sepke, D. a. Shaughnessy, P. T. Springer, R. Tommasini, L. Bernstein, W. Stoeffl, R. Betti, T. R. Boehly, T. C. Sangster, V. Yu. Glebov, P. W. McKenty, S. P. Regan, D. H. Edgell, J. P. Knauer, C. Stoeckl, D. R. 
Harding, S. Batha, G. Grim, H. W. Herrmann, G. Kyrala, M. Wilke, D. C. Wilson, J. Frenje, R. Petrasso, K. Moreno, H. Huang, K. C. Chen, E. Giraldez, J. D. Kilkenny, M. Mauldin, N. Hein, M. Hoppe, a. Nikroo, and R. J. Leeper, The experimental plan for cryogenic layered target implosions on the National Ignition FacilityThe inertial confinement approach to fusion, Physics of Plasmas 18 (2011), no. 5, 051003.

[45] M. J. Edwards, P. K. Patel, J. D. Lindl, L. J. Atherton, S. H. Glenzer, S. W. Haan, J. D. Kilkenny, O. L. Landen, E. I. Moses, a. Nikroo, R. Petrasso, T. C. Sangster, P. T. Springer, S. Batha, R. Benedetti, L. Bernstein, R. Betti, D. L. Bleuel, T. R. Boehly, D. K. Bradley, J. a. Caggiano, D. a. Callahan, P. M. Celliers, C. J. Cerjan, K. C. Chen, D. S. Clark, G. W. Collins, E. L. Dewald, L. Divol, S. Dixit, T. Doeppner, D. H. Edgell, J. E. Fair, M. Farrell, R. J. Fortner, J. Frenje, M. G. Gatu Johnson, E. Giraldez, V. Yu. Glebov, G. Grim, B. a. Hammel, a. V. Hamza, D. R. Harding, S. P. Hatchett, N. Hein, H. W. Herrmann, D. Hicks, D. E. Hinkel, M. Hoppe, W. W. Hsing, N. Izumi, B. Jacoby, O. S. Jones, D. Kalantar, R. Kauffman, J. L. Kline, J. P. Knauer, J. a. Koch, B. J. Kozioziemski, G. Kyrala, K. N. LaFortune, S. Le Pape, R. J. Leeper, R. Lerche, T. Ma, B. J. MacGowan, a. J. MacKinnon, a. Macphee, E. R. Mapoles, M. M. Marinak, M. Mauldin, P. W. McKenty, M. Meezan, P. a. Michel, J. Milovich, J. D. Moody, M. Moran, D. H. Munro, C. L. Olson, K. Opachich, a. E. Pak, T. Parham, H.-S. Park, J. E. Ralph, S. P. Regan, B. Remington, H. Rinderknecht, H. F. Robey, M. Rosen, S. Ross, J. D. Salmonson, J. Sater, D. H. Schneider, F. H. Seguin, S. M. Sepke, D. a. Shaughnessy, V. a. Smalyuk, B. K. Spears, C. Stoeckl, W. Stoeffl, L. Suter, C. a. Thomas, R. Tommasini, R. P. Town, S. V. Weber, P. J. Wegner, K. Widman, M. Wilke, D. C. Wil- 
son, C. B. Yeamans, and a. Zylstra, Progress towards ignition on the National Ignition Facility, Physics of Plasmas 20 (2013), no. 7, 070501.

[46] E. Esarey, C. Schroeder, and W. Leemans, Physics of laser-driven plasma-based electron accelerators, Reviews of Modern Physics 81 (2009), no. 3, 1229-1285.

[47] Eric Esarey, Sally Ride, and Phillip Sprangle, Nonlinear Thomson scattering of intense laser pulses from beams and plasmas, Physical Review E 48 (1993), no. 4, 3003-3021.

[48] T. Esirkepov, M. Borghesi, S. Bulanov, G. Mourou, and T. Tajima, Highly Efficient Relativistic-Ion Generation in the Laser-Piston Regime, Physical Review Letters 92 (2004), no. 17, 2-5.

[49] A. A. Abdo et al., Fermi observations of high-energy gamma-ray emission from grb 080916c, Science 323 (2009), no. 1688.

[50] _ A change in the optical polarization associated with a gamma-ray flare in the blazar 3c279, Nature 463 (2010), no. 919.

[51] Nathaniel Fisch, Theory of current drive in plasmas, Reviews of Modern Physics 59 (1987), no. 1, 175-234.

[52] Nathaniel J Fisch and J. Rax, Free energy in plasmas under wave-induced diffusion, Physics of Fluids B: Plasma Physics 5 (1993), no. 6, 1754-1759.

[53] F. Fiuza, R. Fonseca, J. Tonge, W. Mori, and L. Silva, Weibel-InstabilityMediated Collisionless Shocks in the Laboratory with Ultraintense Lasers, Physical Review Letters 108 (2012), no. 23, 1-5. 
[54] F. Fiuza, A. Stockem, E. Boella, R. Fonseca, L. Silva, D. Haberberger, S. Tochitsky, C. Gong, W. Mori, and C. Joshi, Laser-Driven Shock Acceleration of Monoenergetic Ion Beams, Physical Review Letters 109 (2012), no. 21, 215001.

[55] R A Fonseca, S F Martins, L O Silva, J W Tonge, F S Tsung, and W B Mori, One-to-one direct modeling of experiments and astrophysical scenarios: pushing the envelope on kinetic plasma simulations, Plasma Physics and Controlled Fusion 50 (2008), no. 12, 124034.

[56] W. Fox, G. Fiksel, A. Bhattacharjee, P.-Y. Chang, K. Germaschewski, S. X. Hu, and P. M. Nilson, Filamentation Instability of Counterstreaming Laser-Driven Plasmas, Physical Review Letters 111 (2013), no. 22, 225002.

[57] Richard R Freeman, Fast Ignition Review, 2011, pp. 1-36.

[58] A Friedman, SE Parker, SL Ray, and CK Birdsall, Multi-scale particle-in-cell plasma simulation, Journal of Computational Physics 96 (1991), no. 1, 54-70.

[59] J. Fuchs, Y. Sentoku, S. Karsch, J. Cobble, P. Audebert, a. Kemp, a. Nikroo, P. Antici, E. Brambrink, a. Blazevic, E. Campbell, J. Fernández, J.-C. Gauthier, M. Geissel, M. Hegelich, H. Pépin, H. Popescu, N. Renard-LeGalloudec, M. Roth, J. Schreiber, R. Stephens, and T. Cowan, Comparison of Laser Ion Acceleration from the Front and Rear Surfaces of Thin Foils, Physical Review Letters 94 (2005), no. 4, 1-4.

[60] L. Gao, P. M. Nilson, I. V. Igumenschev, G. Fiksel, R. Yan, J. R. Davies, D. Martinez, V. Smalyuk, M. G. Haines, E. G. Blackman, D. H. Froula, R. Betti, and D. D. Meyerhofer, Observation of Self-Similarity in the Magnetic Fields Generated by the Ablative Nonlinear Rayleigh-Taylor Instability, Physical Review Letters 110 (2013), no. 18, 185003. 
[61] L. Gao, P. M. Nilson, I. V. Igumenschev, S. X. Hu, J. R. Davies, C. Stoeckl, M. G. Haines, D. H. Froula, R. Betti, and D. D. Meyerhofer, Magnetic Field Generation by the Rayleigh-Taylor Instability in Laser-Driven Planar Plastic Targets, Physical Review Letters 109 (2012), no. 11, 115001.

[62] P Gibbon, A A Andreev, and K Yu Platonov, A kinematic model of relativistic laser absorption in an overdense plasma, Plasma Physics and Controlled Fusion 54 (2012), no. 4, 045001.

[63] Paul Gibbon, Short pulse laser interactions with matter, Imperial College Press London, 2005.

[64] V. Ginzburg, Pergamon, London, 1970.

[65] S. Glenzer, B. MacGowan, N. Meezan, P. Adams, J. Alfonso, E. Alger, Z. Alherz, L. Alvarez, S. Alvarez, P. Amick, K. Andersson, S. Andrews, G. Antonini, P. Arnold, D. Atkinson, L. Auyang, S. Azevedo, B. Balaoing, J. Baltz, F. Barbosa, G. Bardsley, D. Barker, a. Barnes, a. Baron, R. Beeler, B. Beeman, L. Belk, J. Bell, P. Bell, R. Berger, M. Bergonia, L. Bernardez, L. Berzins, R. Bettenhausen, L. Bezerides, S. Bhandarkar, C. Bishop, E. Bond, D. Bopp, J. Borgman, J. Bower, G. Bowers, M. Bowers, D. Boyle, D. Bradley, J. Bragg, J. Braucht, D. Brinkerhoff, D. Browning, G. Brunton, S. Burkhart, S. Burns, K. Burns, B. Burr, L. Burrows, R. Butlin, N. Cahayag, D. Callahan, P. Cardinale, R. Carey, J. Carlson, a. Casey, C. Castro, J. Celeste, a. Chakicherla, F. Chambers, C. Chan, H. Chandrasekaran, C. Chang, R. Chapman, K. Charron, Y. Chen, M. Christensen, a. Churby, T. Clancy, B. Cline, L. Clowdus, D. Cocherell, F. Coffield, S. Cohen, R. Costa, J. Cox, G. Curnow, M. Dailey, P. Danforth, R. Darbee, P. Datte, J. Davis, G. Deis, R. Demaret, E. Dewald, 
P. Di Nicola, J. Di Nicola, L. Divol, S. Dixit, D. Dobson, T. Doppner, J. Driscoll, J. Dugorepec, J. Duncan, P. Dupuy, E. Dzenitis, M. Eckart, S. Edson, G. Edwards, M. Edwards, O. Edwards, P. Edwards, J. Ellefson, C. Ellerbee, G. Erbert, C. Estes, W. Fabyan, R. Fallejo, M. Fedorov, B. Felker, J. Fink, M. Finney, L. Finnie, M. Fischer, J. Fisher, B. Fishler, J. Florio, a. Forsman, C. Foxworthy, R. Franks, T. Frazier, G. Frieder, T. Fung, G. Gawinski, C. Gibson, E. Giraldez, S. Glenn, B. Golick, H. Gonzales, S. Gonzales, M. Gonzalez, K. Griffin, J. Grippen, S. Gross, P. Gschweng, G. Gururangan, K. Gu, S. Haan, S. Hahn, B. Haid, J. Hamblen, B. Hammel, a. Hamza, D. Hardy, D. Hart, R. Hartley, C. Haynam, G. Heestand, M. Hermann, G. Hermes, D. Hey, R. Hibbard, D. Hicks, D. Hinkel, D. Hipple, J. Hitchcock, D. Hodtwalker, J. Holder, J. Hollis, G. Holtmeier, S. Huber, a. Huey, D. Hulsey, S. Hunter, T. Huppler, M. Hutton, N. Izumi, J. Jackson, M. Jackson, K. Jancaitis, D. Jedlovec, B. Johnson, M. Johnson, T. Johnson, M. Johnston, O. Jones, D. Kalantar, J. Kamperschroer, R. Kauffman, G. Keating, L. Kegelmeyer, S. Kenitzer, J. Kimbrough, K. King, R. Kirkwood, J. Klingmann, K. Knittel, T. Kohut, K. Koka, S. Kramer, J. Krammen, K. Krauter, G. Krauter, E. Krieger, J. Kroll, K. La Fortune, L. Lagin, V. Lakamsani, O. Landen, S. Lane, a. Langdon, S. Langer, N. Lao, D. Larson, D. Latray, G. Lau, S. Le Pape, B. Lechleiter, Y. Lee, T. Lee, J. Li, J. Liebman, J. Lindl, S. Locke, H. Loey, R. London, F. Lopez, D. Lord, R. Lowe-Webb, J. Lown, a. Ludwigsen, N. Lum, R. Lyons, T. Ma, a. MacKinnon, M. Magat, D. Maloy, T. Malsbury, G. Markham, R. Marquez, a. Marsh, C. Marshall, S. Marshall, I. Maslennikov, D. Mathisen, G. Mauger, M. Mauvais, J. McBride, T. McCarville, J. McCloud, a. McGrew, B. McHale, a. MacPhee, J. Meeker, J. Merill, E. Mertens, P. Michel, M. Miller, T. Mills, J. Milovich, R. Miramontes, R. Montesanti, M. Montoya, J. Moody, K. Moreno, J. Morris, K. Morriston, 
J. Nelson, M. Neto, J. Neumann, E. Ng, Q. Ngo, B. Olejniczak, R. Olson, N. Orsi, M. Owens, E. Padilla, T. Pannell, T. Parham, R. Patterson, G. Pavel, R. Prasad, D. Pendlton, F. Penko, B. Pepmeier, D. Petersen, T. Phillips, D. Pigg, K. Piston, K. Pletcher, C. Powell, H. Radousky, B. Raimondi, J. Ralph, R. Rampke, R. Reed, W. Reid, V. Rekow, J. Reynolds, J. Rhodes, M. Richardson, R. Rinnert, B. Riordan, a. Rivenes, a. Rivera, C. Roberts, J. Robinson, R. Robinson, S. Robison, O. Rodriguez, S. Rogers, M. Rosen, G. Ross, M. Runkel, a. Runtal, R. Sacks, S. Sailors, J. Salmon, J. Salmonson, R. Saunders, J. Schaffer, T. Schindler, M. Schmitt, M. Schneider, K. Segraves, M. Shaw, M. Sheldrick, R. Shelton, M. Shiflett, S. Shiromizu, M. Shor, L. Silva, S. Silva, K. Skulina, D. Smauley, B. Smith, L. Smith, a. Solomon, S. Sommer, J. Soto, N. Spafford, D. Speck, P. Springer, M. Stadermann, F. Stanley, T. Stone, E. Stout, P. Stratton, R. Strausser, L. Suter, W. Sweet, M. Swisher, J. Tappero, J. Tassano, J. Taylor, E. Tekle, C. Thai, C. Thomas, a. Thomas, a. Throop, G. Tietbohl, J. Tillman, R. Town, S. Townsend, K. Tribbey, D. Trummer, J. Truong, J. Vaher, M. Valadez, P. Van Arsdall, a. Van Prooyen, E. Vergel de Dios, M. Vergino, S. Vernon, J. Vickers, G. Villanueva, M. Vitalich, S. Vonhof, F. Wade, R. Wallace, C. Warren, a. Warrick, J. Watkins, S. Weaver, P. Wegner, M. Weingart, J. Wen, K. White, P. Whitman, K. Widmann, C. Widmayer, K. Wilhelmsen, E. Williams, W. Williams, L. Willis, E Wilson, B. Wilson, M. Witte, K. Work, P. Yang, B. Young, K. Youngblood, R. Zacharias, T. Zaleski, P. Zapata, H. Zhang, J. Zielinski, J. Kline, G. Kyrala, C. Niemann, J. Kilkenny, a. Nikroo, B. Van Wonterghem, L. Atherton, and E. Moses, Demonstration of Ignition Radiation Temperatures in Indirect-Drive Inertial Confinement Fusion Hohlraums, Physical Review Letters 106 (2011), no. 8, 1-5. 
[66] S H Glenzer, B J MacGowan, P Michel, N B Meezan, L J Suter, S N Dixit, J L Kline, G a Kyrala, D K Bradley, D a Callahan, E L Dewald, L Divol, E Dzenitis, M J Edwards, a V Hamza, C a Haynam, D E Hinkel, D H Kalantar, J D Kilkenny, O L Landen, J D Lindl, S LePape, J D Moody, a Nikroo, T Parham, M B Schneider, R P J Town, P Wegner, K Widmann, P Whitman, B K F Young, B Van Wonterghem, L J Atherton, and E I Moses, Symmetric inertial confinement fusion implosions at ultra-high laser energies., Science (New York, N.Y.) 327 (2010), no. 5970, 1228-31.

[67] S. Gordienko, A. Pukhov, O. Shorokhov, and T. Baeva, Coherent Focusing of High Harmonics: A New Way Towards the Extreme Intensities, Physical Review Letters 94 (2005), no. 10, 103903.

[68] O. Gotchev, P. Chang, J. Knauer, D. Meyerhofer, O. Polomarov, J. Frenje, C. Li, M. Manuel, R. Petrasso, J. Rygg, F. Séguin, and R. Betti, Laser-Driven Magnetic-Flux Compression in High-Energy-Density Plasmas, Physical Review Letters 103 (2009), no. 21, 215004.

[69] G Gregori, a Ravasio, C D Murphy, K Schaar, a Baird, a R Bell, a BenuzziMounaix, R Bingham, C Constantin, R P Drake, M Edwards, E T Everson, C D Gregory, Y Kuramitsu, W Lau, J Mithen, C Niemann, H-S Park, B a Remington, B Reville, a P L Robinson, D D Ryutov, Y Sakawa, S Yang, N C Woolsey, M Koenig, and F Miniati, Generation of scaled protogalactic seed magnetic fields in laser-produced shock waves., Nature 481 (2012), no. 7382, $480-3$.

[70] C. D. Gregory, B. Loupias, J. Waugh, S. Dono, S. Bouquet, E. Falize, Y. Kuramitsu, C. Michaut, W. Nazarov, S. a. Pikuz, Y. Sakawa, N. C. Woolsey, and 
M. Koenig, Laser-driven plasma jets propagating in an ambient gas studied with optical and proton diagnostics, Physics of Plasmas 17 (2010), no. 5, 052708.

[71] Dan Haberberger, Sergei Tochitsky, Frederico Fiuza, Chao Gong, Ricardo A Fonseca, Luis O Silva, Warren B Mori, and Chan Joshi, Collisionless shocks in laser-produced plasma generate monoenergetic high-energy proton beams, Nature Physics 8 (2011), no. 1, 95-99.

[72] M. Haines, M. Wei, F. Beg, and R. Stephens, Hot-Electron Temperature and Laser-Light Absorption in Fast Ignition, Physical Review Letters 102 (2009), no. $4,1-4$.

[73] R. D. Hazeltine and S. M. Mahajan, Generalization of collisional fluid theory to long mean-free-path and relativistic motion, Physics of Plasmas 9 (2002), no. 8, 3341.

[74] B M Hegelich, B J Albright, J Cobble, K Flippo, S Letzring, M Paffett, H Ruhl, J Schreiber, R K Schulze, and J C Fernández, Laser acceleration of quasimonoenergetic MeV ion beams., Nature 439 (2006), no. 7075, 441-4.

[75] Roger W Hockney and James W Eastwood, Computer simulation using particles, CRC Press, 2010.

[76] G.E. Hogan, K.J. Adams, K.R. Alrick, J.F. Amann, J.G. Boissevain, M.L. Crow, S.B. Cushing, J.C. Eddelman, C.J. Espinoza, T.T. Fife, R.A. Gallegos, J. Gomez, T.J. Gorman, N.T. Gray, V.H. Holmes, S.A. Jaramillo, N.S.P. King, J.N. Knudson, R.K. London, R.P. Lopez, J.B. McClelland, F.E. Merrill, K.B. Morley, C.L. Morris, C.T. Mottershead, K.L. Mueller, F.A. Neri, D.M. Numkena, P.D. Pazuchanics, C Pillai, R.E. Prael, C.M. Riedel, J.S. Sarracino, A Saunders, H.L. Stacy, B.E. Takala, H.A. Thiessen, H.E. Tucker, P.L. 
Walstrom, G.J. Yates, H.-J. Ziock, J.D. Zumbro, E Ables, M.B. Aufderheide, P.D. Barnes, R.M. Bionta, D.H. Fujino, E.P. Hartouni, H.-S. Park, R Soltz, D.M. Wright, S Balzer, P.A. Flores, R.T. Thompson, A Pendzick, R Prigl, J Scaduto, E.T. Schwaner, and J.M. O’Donnell, Proton radiography, Proceedings of the 1999 Particle Accelerator Conference (Cat. No.99CH36366), vol. 1, IEEE, 1999, pp. 579-583.

[77] C M Huntington, F Fiuza, J S Ross, A B Zylstra, R P Drake, D. H. Froula, G. Gregori, N. L. Kugland, C. C. Kuranz, M. C. Levy, C. K. Li, J. Meinecke, T. Morita, R. Petrasso, C. Plechaty, B. A. Remington, D. D. Ryutov, Y. Sakawa, A. Spitkovsky, H. Takabe, and H. S. Park, Observation of magnetic field generation via the Weibel instability in interpenetrating plasma flows, (2013), 1-13.

[78] O. A. Hurricane, D. A. Callahan, D. T. Casey, P. M. Celliers, C. Cerjan, E. L. Dewald, T. R. Dittrich, T. Döppner, D. E. Hinkel, L. F. Berzak Hopkins, J. L. Kline, S. Le Pape, T. Ma, a. G. MacPhee, J. L. Milovich, A. Pak, H.-S. Park, P. K. Patel, B. a. Remington, J. D. Salmonson, P. T. Springer, and R. Tommasini, Fuel gain exceeding unity in an inertially confined fusion implosion, Nature (2014).

[79] John David Jackson and Ronald F Fox, Classical electrodynamics, American Journal of Physics 67 (1999), no. 9, 841-842.

[80] S. Jiang, A. G. Krygier, D. W. Schumacher, K. U. Akli, and R. R. Freeman, Effects of front-surface target structures on properties of relativistic laser-plasma electrons, Physical Review E 89 (2014), no. 1, 013106.

[81] T. Johzaki, H. Nagatomo, a. Sunahara, H.-B. Cai, H. Sakagami, Y. Nakao, and K. Mima, Pre-plasma effects on core heating and enhancing heating efficiency 
by extended double cone for FIREX, Nuclear Fusion 51 (2011), no. 7, 073022.

[82] S. Kar, M. Borghesi, P. Audebert, a. Benuzzi-Mounaix, T. Boehly, D. Hicks, M. Koenig, K. Lancaster, S. Lepape, a. Mackinnon, P. Norreys, P. Patel, and L. Romagnani, Modeling of laser-driven proton radiography of dense matter, High Energy Density Physics 4 (2008), no. 1-2, 26-40.

[83] A. Kemp and L. Divol, Interaction Physics of Multipicosecond Petawatt Laser Pulses with Overdense Plasma, Physical Review Letters 109 (2012), no. 19, 195005.

[84] A. Kemp, Y. Sentoku, and M. Tabak, Hot-Electron Energy Coupling in Ultraintense Laser-Matter Interaction, Physical Review Letters 101 (2008), no. 7, $8-11$.

[85] _ Hot-electron energy coupling in ultraintense laser-matter interaction, Physical Review E 79 (2009), no. 6, 066406.

[86] A. J. Kemp, B. I. Cohen, and L. Divol, Integrated kinetic simulation of laserplasma interactions, fast-electron generation, and transport in fast ignition, Physics of Plasmas 17 (2010), no. 5, 056702.

[87] A. J. Kemp, Y. Sentoku, V. Sotnikov, and S. Wilks, Collisional Relaxation of Superthermal Electrons Generated by Relativistic Laser Pulses in Dense Plasma, Physical Review Letters 97 (2006), no. 23, 8-11.

[88] A.J. Kemp, F. Fiuza, A. Debayle, T. Johzaki, W.B. Mori, P.K. Patel, Y. Sentoku, and L.O. Silva, Laserplasma interactions for fast ignition, Nuclear Fusion 54 (2014), no. 5, 054002. 
[89] G. E. Kemp, a. Link, Y. Ping, D. W. Schumacher, R. R. Freeman, and P. K. Patel, Coupling of laser energy into hot-electrons in high-contrast relativistic laser-plasma interactions, Physics of Plasmas 20 (2013), no. 3, 033104.

[90] M. H. Key, Status of and prospects for the fast ignition inertial fusion concept, Physics of Plasmas 14 (2007), no. 5, 055502.

[91] M. H. Key, M. D. Cable, T. E. Cowan, K. G. Estabrook, B. a. Hammel, S. P. Hatchett, E. a. Henry, D. E. Hinkel, J. D. Kilkenny, J. a. Koch, W. L. Kruer, a. B. Langdon, B. F. Lasinski, R. W. Lee, B. J. MacGowan, A. MacKinnon, J. D. Moody, M. J. Moran, a. a. Offenberger, D. M. Pennington, M. D. Perry, T. J. Phillips, T. C. Sangster, M. S. Singh, M. a. Stoyer, M. Tabak, G. L. Tietbohl, M. Tsukamoto, K. Wharton, and S. C. Wilks, Hot electron production and heating by hot electrons in fast ignitor research, Physics of Plasmas 5 (1998), no. 5,1966 .

[92] T. Kluge, T. Cowan, A. Debus, U. Schramm, K. Zeil, and M. Bussmann, Electron Temperature Scaling in Laser Interaction with Solids, Physical Review Letters 107 (2011), no. 20, 205003.

[93] R Kodama, Y Sentoku, Z L Chen, G R Kumar, S P Hatchett, Y Toyama, T E Cowan, R R Freeman, J Fuchs, Y Izawa, M H Key, Y Kitagawa, K Kondo, T Matsuoka, H Nakamura, M Nakatsutsumi, P a Norreys, T Norimatsu, R a Snavely, R B Stephens, M Tampo, K a Tanaka, and T Yabuuchi, Plasma devices to guide and collimate a high density of MeV electrons., Nature 432 (2004), no. $7020,1005-8$.

[94] D. E. Harris \& H. Krawczynski, X-ray emission from extragalactic jets, Ann. Rev. Astron. Astr. 44 (2006), no. 463. 
[95] W. L. Kruer and Kent Estabrook, JB heating by very intense laser light, Physics of Fluids 28 (1985), no. 1, 430.

[96] N L Kugland, J S Ross, P.-Y. Chang, R P Drake, G Fiksel, D. H. Froula, S. H. Glenzer, G. Gregori, M. Grosskopf, C. Huntington, M. Koenig, Y. Kuramitsu, C. Kuranz, M. C. Levy, E. Liang, D. Martinez, J. Meinecke, F. Miniati, T. Morita, A. Pelka, C. Plechaty, R. Presura, A. Ravasio, B. A. Remington, B. Reville, D. D. Ryutov, Y. Sakawa, A. Spitkovsky, H. Takabe, and H.-S. Park, Visualizing electromagnetic fields in laser-produced counter-streaming plasma experiments for collisionless shock laboratory astrophysics, Physics of Plasmas 20 (2013), no. 5, 056313.

[97] N. L. Kugland, D. D. Ryutov, P-Y. Chang, R. P. Drake, G. Fiksel, D. H. Froula, S. H. Glenzer, G. Gregori, M. Grosskopf, M. Koenig, Y. Kuramitsu, C. Kuranz, M. C. Levy, E. Liang, J. Meinecke, F. Miniati, T. Morita, A. Pelka, C. Plechaty, R. Presura, A. Ravasio, B. a. Remington, B. Reville, J. S. Ross, Y. Sakawa, A. Spitkovsky, H. Takabe, and H-S. Park, Self-organized electromagnetic field structures in laser-produced counter-streaming plasmas, Nature Physics 8 (2012), no. 11, 809-812.

[98] N. L. Kugland, D. D. Ryutov, C. Plechaty, J. S. Ross, and H.-S. Park, Invited Article: Relation between electric and magnetic field structures and their protonbeam images, Review of Scientific Instruments 83 (2012), no. 10, 101301.

[99] O. L. Landen, J. Edwards, S. W. Haan, H. F. Robey, J. Milovich, B. K. Spears, S. V. Weber, D. S. Clark, J. D. Lindl, B. J. MacGowan, E. I. Moses, J. Atherton, P. a. Amendt, T. R. Boehly, D. K. Bradley, D. G. Braun, D. a. Callahan, P. M. Celliers, G. W. Collins, E. L. Dewald, L. Divol, J. a. Frenje, S. H. Glenzer, 
a. Hamza, B. a. Hammel, D. G. Hicks, N. Hoffman, N. Izumi, O. S. Jones, J. D. Kilkenny, R. K. Kirkwood, J. L. Kline, G. a. Kyrala, M. M. Marinak, N. Meezan, D. D. Meyerhofer, P. Michel, D. H. Munro, R. E. Olson, a. Nikroo, S. P. Regan, L. J. Suter, C. a. Thomas, and D. C. Wilson, Capsule implosion optimization during the indirect-drive National Ignition Campaign, Physics of Plasmas 18 (2011), no. 5, 051002.

[100] Sebastien Le Pape, Ying Yin Tsui, Andrew Macphee, Daniel Hey, Pravesh Patel, Andrew Mackinnon, Mike Key, Mingsheng Wei, Tammy Ma, Farhat N Beg, Rich Stephens, Kramer Akli, Tony Link, Linn Van-Woerkom, and Rick R Freeman, Characterization of the preformed plasma for high-intensity laserplasma interaction., Optics letters 34 (2009), no. 19, 2997-9.

[101] Sergey Lebedev, Order from disorder, Nature Physics (2012), 1-3.

[102] W. P. Leemans and E. Esarey, Laser-driven plasma-wave electron accelerators, Physics Today (2009).

[103] W. P. Leemans, B. Nagler, A. J. Gonsalves, Cs. Tóth, K. Nakamura, C. G. R. Geddes, E. Esarey, C. B. Schroeder, and S. M. Hooker, GeV electron beams from a centimetre-scale accelerator, Nature Physics 2 (2006), no. 10, 696-699.

[104] M. C. Levy, A. J. Kemp, S. C. Wilks, L. Divol, and M. G. Baring, Focusing of intense subpicosecond laser pulses in wedge targets, Physics of Plasmas 18 (2011), no. 10, 103110.

[105] M. C. Levy, D. D. Ryutov, S. C. Wilks, J. S. Ross, C. M. Huntington, H-. S. Park, and M. G. Baring, Development of interpretive simulation tool for the proton radiography technique, Submitted (2014). 
[106] M C Levy, S C Wilks, M Tabak, and M G Baring, Conservation laws and conversion efficiency in ultraintense laser-overdense plasma interactions, Physics of Plasmas 20 (2013), no. 10, 103101.

[107] M. C. Levy, S. C. Wilks, M. Tabak, and M. G. Baring, Hot electron divergence in the kinematic analysis of relativistic light on solids, In press, Journal of Physics: Conf. Series (2014).

[108] M C Levy, S C Wilks, M Tabak, S B Libby, and M G Baring, Kinematic Constraints on Absorption of Ultraintense Laser Light, (2013), 1-6.

[109] Matthew C. Levy, Scott C. Wilks, Max Tabak, Stephen B. Libby, and Matthew G. Baring, Petawatt laser absorption bounded, Nature Communications 5 (2014), no. May, 1-6.

[110] M.C. Levy, S.C. Wilks, and M.G. Baring, Accelerating piston action and plasma heating in high-energy density laser plasma interactions, High Energy Density Physics 9 (2013), no. 1, 198-203.

[111] C. Li, F. Séguin, J. Frenje, J. Rygg, R. Petrasso, R. Town, P. Amendt, S. Hatchett, O. Landen, A. Mackinnon, P. Patel, V. Smalyuk, T. Sangster, and J. Knauer, Measuring E and B Fields in Laser-Produced Plasmas with Monoenergetic Proton Radiography, Physical Review Letters 97 (2006), no. 13, 135003.

[112] C. Li, F. Séguin, J. Frenje, J. Rygg, R. Petrasso, R. Town, P. Amendt, S. Hatchett, O. Landen, a. Mackinnon, P. Patel, M. Tabak, J. Knauer, T. Sangster, and V. Smalyuk, Observation of the Decay Dynamics and Instabilities of Megagauss Field Structures in Laser-Produced Plasmas, Physical Review Letters 99 (2007), no. 1, 015001. 
[113] C. Li, F. Séguin, J. Frenje, J. Rygg, R. Petrasso, R. Town, O. Landen, J. Knauer, and V. Smalyuk, Observation of Megagauss-Field Topology Changes due to Magnetic Reconnection in Laser-Produced Plasmas, Physical Review Letters 99 (2007), no. 5, 055001.

[114] C K Li, F H Séguin, J A Frenje, M Manuel, R D Petrasso, V a Smalyuk, R Betti, J Delettrez, J P Knauer, F Marshall, D D Meyerhofer, D Shvarts, C Stoeckl, W Theobald, J R Rygg, O L Landen, R P J Town, P a Amendt, C a Back, and J D Kilkenny, Study of direct-drive capsule implosions in inertial confinement fusion with proton radiography, Plasma Physics and Controlled Fusion 51 (2009), no. 1, 014003.

[115] C K Li, F H Séguin, J a Frenje, M Rosenberg, R D Petrasso, P a Amendt, J a Koch, O L Landen, H S Park, H F Robey, R P J Town, a Casner, F Philippe, R Betti, J P Knauer, D D Meyerhofer, C a Back, J D Kilkenny, and a Nikroo, Charged-particle probing of x-ray-driven inertial-fusion implosions., Science (New York, N.Y.) 327 (2010), no. 5970, 1231-5.

[116] _ Charged-particle probing of x-ray-driven inertial-fusion implosions., Science (New York, N.Y.) 327 (2010), no. 5970, 1231-5.

[117] C. K. Li, F. H. Séguin, J. a. Frenje, M. J. Rosenberg, H. G. Rinderknecht, a. B. Zylstra, R. D. Petrasso, P. a. Amendt, O. L. Landen, a. J. Mackinnon, R. P. J. Town, S. C. Wilks, R. Betti, D. D. Meyerhofer, J. M. Soures, J. Hund, J. D. Kilkenny, and a. Nikroo, Impeding Hohlraum Plasma Stagnation in InertialConfinement Fusion, Physical Review Letters 108 (2012), no. 2, 025001.

[118] C. K. Li, F. H. Seguin, J. A. Frenje, M. Manuel, D. Casey, N. Sinenian, R. D. Petrasso, P. A. Amendt, O. L. Landen, J. R. Rygg, R. P. J. Town, R. Betti, 
J. Delettrez, J. P. Knauer, F. Marshall, D. D. Meyerhofer, T. C. Sangster, D. Shvarts, V. A. Smalyuk, J. M. Soures, C. A. Back, J. D. Kilkenny, and A. Nikroo, Proton radiography of dynamic electric and magnetic fields in laserproduced high-energy-density plasmas, Physics of Plasmas 16 (2009), no. 5, 056304.

[119] C. K. Li, F. H. Seguin, J. a. Frenje, J. R. Rygg, R. D. Petrasso, R. P. J. Town, P. a. Amendt, S. P. Hatchett, O. L. Landen, a. J. Mackinnon, P. K. Patel, V. a. Smalyuk, J. P. Knauer, T. C. Sangster, and C. Stoeckl, Monoenergetic proton backlighter for measuring $E$ and $B$ fields and for radiographing implosions and high-energy density plasmas (invited), Review of Scientific Instruments 77 (2006), no. 10, 10E725.

[120] G Li, R Yan, and C Ren, Laser Channeling in Millimeter-Scale Underdense Plasmas of Fast-Ignition Targets, Physical Review Letters 125002 (2008), no. March, 1-4.

[121] E. Liang, S. C. Wilks, and M. Tabak, Pair Production by Ultraintense Lasers, Physical Review Letters 81 (1998), no. 22, 4887-4890.

[122] J. D. Lindl and E. I. Moses, Special Topic: Plans for the National Ignition Campaign (NIC) on the National Ignition Facility (NIF): On the threshold of initiating ignition experiments, Physics of Plasmas 18 (2011), no. 5, 050901.

[123] John Lindl, Otto Landen, John Edwards, and Ed Moses, Review of the National Ignition Campaign 2009-2012, Physics of Plasmas 21 (2014), no. 2, 020501.

[124] John D. Lindl, Peter Amendt, Richard L. Berger, S. Gail Glendinning, Siegfried H. Glenzer, Steven W. Haan, Robert L. Kauffman, Otto L. Landen, 
and Laurence J. Suter, The physics basis for ignition using indirect-drive targets on the National Ignition Facility, Physics of Plasmas 11 (2004), no. 2, 339.

[125] B. Loupias, C. D. Gregory, E. Falize, J. Waugh, D. Seiichi, S. Pikuz, Y. Kuramitsu, a. Ravasio, S. Bouquet, C. Michaut, P. Barroso, M. RabecleGloahec, W. Nazarov, H. Takabe, Y. Sakawa, N. Woolsey, and M. Koenig, Experimental results to study astrophysical plasma jets using Intense Lasers, Astrophysics and Space Science 322 (2009), no. 1-4, 25-29.

[126] M. Lyutikov, On the nature of eclipses in binary pulsar J0737-3039, Monthly Notices of the Royal Astronomical Society 353 (2004), no. 4, 1095-1106.

[127] G. M. Madejski \& J.-P. Lasota M. Sikora, M. C. Begelman, Are quasar jets dominated by poynting flux?, Astrophys. J. 625 (2005), no. 72.

[128] T. Ma, H. Sawada, P. Patel, C. Chen, L. Divol, D. Higginson, a. Kemp, M. Key, D. Larson, S. Le Pape, a. Link, a. MacPhee, H. McLean, Y. Ping, R. Stephens, S. Wilks, and F. Beg, Hot Electron Temperature and Coupling Efficiency Scaling with Prepulse for Cone-Guided Fast Ignition, Physical Review Letters 108 (2012), no. 11, 1-5.

[129] Andrea Macchi, A Superintense Laser-Plasma Interaction Theory Primer, SpringerBriefs in Physics, Springer Netherlands, Dordrecht, 2013.

[130] Andrea Macchi, Marco Borghesi, and Matteo Passoni, Ion acceleration by superintense laser-plasma interaction, Reviews of Modern Physics 85 (2013), no. 2, 751-793.

[131] Andrea Macchi, Federica Cattani, Tatiana Liseykina, and Fulvio Cornolti, Laser 
Acceleration of Ion Bunches at the Front Surface of Overdense Plasmas, Physical Review Letters 94 (2005), no. 16, 2-5.

[132] A. Mackinnon, P. Patel, M. Borghesi, R. Clarke, R. Freeman, H. Habara, S. Hatchett, D. Hey, D. Hicks, S. Kar, M. Key, J. King, K. Lancaster, D. Neely, A. Nikkro, P. Norreys, M. Notley, T. Phillips, L. Romagnani, R. Snavely, R. Stephens, and R. Town, Proton Radiography of a Laser-Driven Implosion, Physical Review Letters 97 (2006), no. 4, 045001.

[133] A. J. Mackinnon, P. K. Patel, R. P. Town, M. J. Edwards, T. Phillips, S. C. Lerner, D. W. Price, D. Hicks, M. H. Key, S. Hatchett, S. C. Wilks, M. Borghesi, L. Romagnani, S. Kar, T. Toncian, G. Pretzler, O. Willi, M. Koenig, E. Martinolli, S. Lepape, A. Benuzzi-Mounaix, P. Audebert, J. C. Gauthier, J. King, R. Snavely, R. R. Freeman, and T. Boehlly, Proton radiography as an electromagnetic field and density perturbation diagnostic (invited), Review of Scientific Instruments 75 (2004), no. 10, 3531.

[134] A. G. MacPhee, L. Divol, A. J. Kemp, K. U. Akli, F. N. Beg, C. D. Chen, H. Chen, D. S. Hey, R. J. Fedosejevs, R. R. Freeman, M. Henesian, M. H. Key, S. Le Pape, A. Link, T. Ma, A. J. Mackinnon, V. M. Ovchinnikov, P. K. Patel, T. W. Phillips, R. B. Stephens, M. Tabak, R. Town, Y. Y. Tsui, L. D. Van Woerkom, M. S. Wei, and S. C. Wilks, Limitation on Prepulse Level for ConeGuided Fast-Ignition Inertial Confinement Fusion, Physical Review Letters 104 (2010), no. 5, 1-4.

[135] a Maksimchuk, S Gu, K Flippo, and D Umstadter, Forward Ion Acceleration in Thin Films Driven by a High-Intensity Laser, Physical Review Letters 84 (2000), no. 18, 4108-4111. 
[136] Victor Malka, Jérôme Faure, Yann A Gauduel, Erik Lefebvre, Antoine Rousse, and Kim Ta Phuoc, Principles and applications of compact laserplasma accelerators, Nature Physics 4 (2008), no. 6, 447-453.

[137] M. J.-E. Manuel, C. K. Li, F. H. Séguin, J. Frenje, D. T. Casey, R. D. Petrasso, S. X. Hu, R. Betti, J. D. Hager, D. D. Meyerhofer, and V. a. Smalyuk, First Measurements of Rayleigh-Taylor-Induced Magnetic Fields in Laser-Produced Plasmas, Physical Review Letters 108 (2012), no. 25, 255006.

[138] M. J.-E. Manuel, N. Sinenian, F. H. Seguin, C. K. Li, J. a. Frenje, H. G. Rinderknecht, D. T. Casey, a. B. Zylstra, R. D. Petrasso, and F. N. Beg, Mapping return currents in laser-generated Z-pinch plasmas using proton deflectometry, Applied Physics Letters 100 (2012), no. 20, 203505.

[139] M J-E Manuel, a B Zylstra, H G Rinderknecht, D T Casey, M J Rosenberg, N Sinenian, C K Li, J a Frenje, F H Séguin, and R D Petrasso, Source characterization and modeling development for monoenergetic-proton radiography experiments on OMEGA., The Review of scientific instruments 83 (2012), no. 6, 063506.

[140] J. May, J. Tonge, F. Fiuza, R. a. Fonseca, L. O. Silva, C. Ren, and W. B. Mori, Mechanism of generating fast electrons by an intense laser at a steep overdense interface, Physical Review E 84 (2011), no. 2, 025401.

[141] N. B. Meezan, L. J. Atherton, D. a. Callahan, E. L. Dewald, S. Dixit, E. G. Dzenitis, M. J. Edwards, C. a. Haynam, D. E. Hinkel, O. S. Jones, O. Landen, R. a. London, P. a. Michel, J. D. Moody, J. L. Milovich, M. B. Schneider, C. a. Thomas, R. P. J. Town, a. L. Warrick, S. V. Weber, K. Widmann, S. H. Glenzer, L. J. Suter, B. J. MacGowan, J. L. Kline, G. a. Kyrala, and a. Nikroo, National 
Ignition Campaign Hohlraum energetics, Physics of Plasmas 17 (2010), no. 5, 056304 .

[142] Sanjay Mehrotra, On the implementation of a primal-dual interior point method, SIAM Journal on Optimization 2 (1992), no. 4, 575-601.

[143] J. Meinecke, H. W. Doyle, F. Miniati, a. R. Bell, R. Bingham, R. Crowston, R. P. Drake, M. Fatenejad, M. Koenig, Y. Kuramitsu, C. C. Kuranz, D. Q. Lamb, D. Lee, M. J. MacDonald, C. D. Murphy, H-S. Park, a. Pelka, a. Ravasio, Y. Sakawa, a. a. Schekochihin, a. Scopatz, P. Tzeferacos, W. C. Wan, N. C. Woolsey, R. Yurchak, B. Reville, and G. Gregori, Turbulent amplification of magnetic fields in laboratory laser-produced shock waves, Nature Physics 10 (2014), no. 7, 520-524.

[144] P. Mészáros, Theories of gamma-ray bursts, Ann. Rev. Astron. Astr. 40 (2002), no. 137 .

[145] R. Mishra, Y. Sentoku, and a. J. Kemp, Hot electron generation forming a steep interface in superintense laser-matter interaction, Physics of Plasmas $\mathbf{1 6}$ (2009), no. 11, 112704.

[146] Y. Mizuno, P. Hardee, and K. I. Nishikawa, Relativistic mhd simulations of relativistic jets with raishin, Astrophys. J. 662 (2007), no. 835.

[147] P. Mora, Plasma Expansion into a Vacuum, Physical Review Letters 90 (2003), no. $18,5-8$.

[148] E. I. Moses, R. N. Boyd, B. a. Remington, C. J. Keane, and R. Al-Ayat, The National Ignition Facility: Ushering in a new age for high energy density science, Physics of Plasmas 16 (2009), no. 4, 041006. 
[149] H. Nakamura, B. Chrisman, T. Tanimoto, M. Borghesi, K. Kondo, M. Nakatsutsumi, T. Norimatsu, M. Tampo, K. Tanaka, T. Yabuuchi, Y. Sentoku, and R. Kodama, Superthermal and Efficient-Heating Modes in the Interaction of a Cone Target with Ultraintense Laser Light, Physical Review Letters 102 (2009), no. $4,1-4$.

[150] N. Naumova, T. Schlegel, V. Tikhonchuk, C. Labaune, I. Sokolov, and G. Mourou, Hole Boring in a DT Pellet and Fast-Ion Ignition with Ultraintense Laser Pulses, Physical Review Letters 102 (2009), no. 2, 1-4.

[151] P. Nilson, L. Willingale, M. Kaluza, C. Kamperidis, S. Minardi, M. Wei, P. Fernandes, M. Notley, S. Bandyopadhyay, M. Sherlock, R. Kingham, M. Tatarakis, Z. Najmudin, W. Rozmus, R. Evans, M. Haines, a. Dangor, and K. Krushelnick, Magnetic Reconnection and Plasma Dynamics in Two-Beam Laser-Solid Interactions, Physical Review Letters 97 (2006), no. 25, 255001.

[152] P. Norreys, D Batani, S Baton, F N. Beg, R. Kodama, P.M. Nilson, P. Patel, F. Pérez, J.J. Santos, R.H.H. Scott, V.T. Tikhonchuk, M. Wei, and J. Zhang, Fast electron energy transport in solid density and compressed plasma, Nuclear Fusion 54 (2014), no. 5, 054004.

[153] P. A. Norreys, K. L. Lancaster, C. D. Murphy, H. Habara, S. Karsch, R. J. Clarke, J. Collier, R. Heathcote, C. Hemandez-Gomez, S. Hawkes, D. Neely, M. H. R. Hutchinson, R. G. Evans, M. Borghesi, L. Romagnani, M. Zepf, K. Akli, J. a. King, B. Zhang, R. R. Freeman, a. J. MacKinnon, S. P. Hatchett, P. Patel, R. Snavely, M. H. Key, A. Nikroo, R. Stephens, C. Stoeckl, K. a. Tanaka, T. Norimatsu, Y. Toyama, and R. Kodama, Integrated implosion/heating studies for advanced fast ignition, Physics of Plasmas 11 (2004), 
no. 5,2746 .

[154] JOHN NUCKOLLS, LOWELL WOOD, ALBERT THIESSEN, and GEORGE ZIMMERMAN, Laser Compression of Matter to Super-High Densities: Thermonuclear (CTR) Applications, Nature 239 (1972), no. 5368, 139-142.

[155] Charles Osolin, Harnessing the Power of Light, 2008.

[156] Sebastien Le Pape, Daniel Hey, Pravesh Patel, Andrew Mackinnon, Richard Klein, Bruce Remington, Scott Wilks, Dmitri Ryutov, Steve Moon, and Marc Foord, Proton Radiography of Megagauss Electromagnetic Fields Generated by the Irradiation of a Solid Target by an Ultraintense Laser Pulse, Astrophysics and Space Science 307 (2007), no. 1-3, 341-345.

[157] H.-S. Park, D. M. Chambers, H.-K. Chung, R. J. Clarke, R. Eagleton, E. Giraldez, T. Goldsack, R. Heathcote, N. Izumi, M. H. Key, J. A. King, J. a. Koch, O. L. Landen, A. Nikroo, P. K. Patel, D. F. Price, B. A. Remington, H. F. Robey, R. a. Snavely, D. a. Steinman, R. B. Stephens, C. Stoeckl, M. Storm, M. Tabak, W. Theobald, R. P. J. Town, J. E. Wickersham, and B. B. Zhang, High-energy Ka radiography using high-intensity, short-pulse lasers, Physics of Plasmas 13 (2006), no. 5, 056309.

[158] H.-S. Park, O.a. Hurricane, D.a. Callahan, D.T. Casey, E.L. Dewald, T.R. Dittrich, T. Döppner, D.E. Hinkel, L.F. Berzak Hopkins, S. Le Pape, T. Ma, P.K. Patel, B.a. Remington, H.F. Robey, J.D. Salmonson, and J.L. Kline, HighAdiabat High-Foot Inertial Confinement Fusion Implosion Experiments on the National Ignition Facility, Physical Review Letters 112 (2014), no. 5, 055001.

[159] B. Zhang \& A. Pe'er, Evidence of an initially magnetically dominated outflow in grb 080916c, Astrophys. J. 700 (2009), no. L65. 
[160] F. Pérez, A. Kemp, L. Divol, C. Chen, and P. Patel, Deflection of MeV Electrons by Self-Generated Magnetic Fields in Intense Laser-Solid Interactions, Physical Review Letters 111 (2013), no. 24, 245001.

[161] R. Petrasso, C. Li, F. Seguin, J. Rygg, J. Frenje, R. Betti, J. Knauer, D. Meyerhofer, P. Amendt, D. Froula, O. Landen, P. Patel, J. Ross, and R. Town, Lorentz Mapping of Magnetic Fields in Hot Dense Plasmas, Physical Review Letters 103 (2009), no. 8, 085001.

[162] Y. Ping, a. Kemp, L. Divol, M. Key, P. Patel, K. Akli, F. Beg, S. Chawla, C. Chen, R. Freeman, D. Hey, D. Higginson, L. Jarrott, G. Kemp, a. Link, H. McLean, H. Sawada, R. Stephens, D. Turnbull, B. Westover, and S. Wilks, Dynamics of Relativistic Laser-Plasma Interaction on Solid Targets, Physical Review Letters 109 (2012), no. 14, 1-5.

[163] Y. Ping, R. Shepherd, B. F. Lasinski, M. Tabak, H. Chen, H. Chung, K. Fournier, S. Hansen, A. J. Kemp, D. Liedahl, K. Widmann, S. C. Wilks, W. Rozmus, and M. Sherlock, Absorption of Short Laser Pulses on Solid Targets in the Ultrarelativistic Regime, Physical Review Letters 100 (2008), no. 8, $6-9$.

[164] A. Shemi \& T. Piran, The appearance of cosmic fireballs, Astrophys. J. 365 (1990), no. L55.

[165] T. Piran, Gamma-ray bursts and the fireball model, Phys. Rep. 314 (1999), no. 575 .

[166] A. Pukhov, Strong field interaction of laser radiation, Reports on Progress in Physics 66 (2003), no. 1, 47-101. 
[167] Michael A. Purvis, Vyacheslav N. Shlyaptsev, Reed Hollinger, Clayton Bargsten, Alexander Pukhov, Amy Prieto, Yong Wang, Bradley M. Luther, Liang Yin, Shoujun Wang, and Jorge J. Rocca, Relativistic plasma nanophotonics for ultrahigh energy density physics, Nature Photonics 7 (2013), no. 10, 796-800.

[168] K. Quinn, L. Romagnani, B. Ramakrishna, G. Sarri, M. E. Dieckmann, P. a. Wilson, J. Fuchs, L. Lancia, a. Pipahl, T. Toncian, O. Willi, R. J. Clarke, M. Notley, a. Macchi, and M. Borghesi, Weibel-Induced Filamentation during an Ultrafast Laser-Driven Plasma Expansion, Physical Review Letters 108 (2012), no. 13,135001 .

[169] Bruce Remington, R. Drake, and Dmitri Ryutov, Experimental astrophysics with high power lasers and $Z$ pinches, Reviews of Modern Physics 78 (2006), no. $3,755-807$.

[170] C. Ridgers, C. Brady, R. Duclous, J. Kirk, K. Bennett, T. Arber, a. Robinson, and a. Bell, Dense Electron-Positron Plasmas and Ultraintense $\gamma$ rays from Laser-Irradiated Solids, Physical Review Letters 108 (2012), no. 16, 1-5.

[171] C. P. Ridgers, C. S. Brady, R. Duclous, J. G. Kirk, K. Bennett, T. D. Arber, and a. R. Bell, Dense electron-positron plasmas and bursts of gamma-rays from laser-generated quantum electrodynamic plasmas, Physics of Plasmas 20 (2013), no. 5, 056701.

[172] H. F. Robey, P. M. Celliers, J. L. Kline, a. J. Mackinnon, T. R. Boehly, O. L. Landen, J. H. Eggert, D. Hicks, S. Le Pape, D. R. Farley, M. W. Bowers, K. G. Krauter, D. H. Munro, O. S. Jones, J. L. Milovich, D. Clark, B. K. Spears, R. P. J. Town, S. W. Haan, S. Dixit, M. B. Schneider, E. L. Dewald, K. Widmann, J. D. Moody, T. D. Döppner, H. B. Radousky, a. Nikroo, J. J. 
Kroll, a. V. Hamza, J. B. Horner, S. D. Bhandarkar, E. Dzenitis, E. Alger, E. Giraldez, C. Castro, K. Moreno, C. Haynam, K. N. LaFortune, C. Widmayer, M. Shaw, K. Jancaitis, T. Parham, D. M. Holunga, C. F. Walters, B. Haid, T. Malsbury, D. Trummer, K. R. Coffee, B. Burr, L. V. Berzins, C. Choate, S. J. Brereton, S. Azevedo, H. Chandrasekaran, S. Glenzer, J. a. Caggiano, J. P. Knauer, J. a. Frenje, D. T. Casey, M. Gatu Johnson, F. H. Séguin, B. K. Young, M. J. Edwards, B. M. Van Wonterghem, J. Kilkenny, B. J. MacGowan, J. Atherton, J. D. Lindl, D. D. Meyerhofer, and E. Moses, Precision Shock Tuning on the National Ignition Facility, Physical Review Letters 108 (2012), no. $21,215004$.

[173] A. P. L. Robinson, Production of high energy protons with hole-boring radiation pressure acceleration, Physics of Plasmas 18 (2011), no. 5, 056701.

[174] A P L Robinson, A V Arefiev, and D Neely, Generating Superponderomotive Electrons due to a Non-Wake-Field Interaction between a Laser Pulse and a Longitudinal Electric Field, Physical Review Letters 111 (2013), no. 6, 065002.

[175] A P L Robinson, P Gibbon, M Zepf, S Kar, R G Evans, and C Bellei, Relativistically correct hole-boring and ion acceleration by circularly polarized laser pulses, Plasma Physics and Controlled Fusion 51 (2009), no. 2, 024004.

[176] A. P. L Robinson, M. H. Key, and M. Tabak, Focusing of Relativistic Electrons in Dense Plasma Using a Resistivity-Gradient-Generated Magnetic Switchyard, Physical Review Letters 108 (2012), no. 12, 125004.

[177] A.P.L. Robinson, D.J. Strozzi, J.R. Davies, L. Gremillet, J.J. Honrubia, T. Johzaki, R.J. Kingham, M. Sherlock, and A.a. Solodov, Theory of fast electron transport for fast ignition, Nuclear Fusion 54 (2014), no. 5, 054003. 
[178] L. Romagnani, M. Borghesi, C.a. Cecchetti, S. Kar, P. Antici, P. Audebert, S. Bandhoupadjay, F. Ceccherini, T. Cowan, J. Fuchs, M. Galimberti, L.a. Gizzi, T. Grismayer, R. Heathcote, R. Jung, T.V. Liseykina, a. Macchi, P. Mora, D. Neely, M. Notley, J. Osterholtz, C.a. Pipahl, G. Pretzler, a. Schiavi, G. Schurtz, T. Toncian, P.a. Wilson, and O. Willi, Proton probing measurement of electric and magnetic fields generated by ns and ps laser-matter interactions, Laser and Particle Beams 26 (2008), no. 02, 241-248.

[179] Mordecai D. Rosen, The physics issues that determine inertial confinement fusion target gain and driver requirements: A tutorial, Physics of Plasmas 6 (1999), no. 5, 1690.

[180] J S Ross, S H Glenzer, P Amendt, R Berger, and L Divol, Characterizing counter-streaming interpenetrating plasmas relevant to astrophysical collisionless shocks to astrophysical collisionless shocks a), Physics of Plasmas 056501 (2012).

[181] J. S. Ross, H.-S. Park, R. Berger, L. Divol, N. L. Kugland, W. Rozmus, D. Ryutov, and S. H. Glenzer, Collisionless Coupling of Ion and Electron Temperatures in Counterstreaming Plasma Flows, Physical Review Letters 110 (2013), no. 14, 145005 .

[182] M. Roth, A. Blazevic, M. Geissel, T. Schlegel, T. Cowan, M. Allen, J.-C. Gauthier, P. Audebert, J. Fuchs, J. Meyer-ter Vehn, M. Hegelich, S. Karsch, and A. Pukhov, Energetic ions generated by laser pulses: A detailed study on target properties, Physical Review Special Topics - Accelerators and Beams 5 (2002), no. $6,061301$.

[183] H. Ruhl, Classical Particle Simulations with the PSC code (PSC Manual). 
[184] H. Ruhl, Rinton, Paramus, NJ, 2006.

[185] H Ruhl and P Mulser, Relativistic Vlasov simulation of intense fs laser pulsematter interaction, Physical Letters A 9601 (1995), no. September, 388-392.

[186] J R Rygg, F H Séguin, C K Li, J a Frenje, M J-E Manuel, R D Petrasso, R Betti, J a Delettrez, O V Gotchev, J P Knauer, D D Meyerhofer, F J Marshall, C Stoeckl, and W Theobald, Proton radiography of inertial fusion implosions., Science (New York, N.Y.) 319 (2008), no. 5867, 1223-5.

[187] D. D. Ryutov, F. Fiuza, C. M. Huntington, J. S. Ross, and H.-S. Park, Collisional effects in the ion Weibel instability for two counter-propagating plasma streams, Physics of Plasmas 21 (2014), no. 3, 032701.

[188] D D Ryutov, N L Kugland, M C Levy, C Plechaty, J S Ross, and H S Park, Magnetic field advection in two interpenetrating plasma streams, Physics of Plasmas 20 (2013), no. 3, 032703.

[189] G Sarri, M Borghesi, C A Cecchetti, L Romagnani, R Jung, O Willi, D J Hoarty, R M Stevenson, C. R.D. Brown, S. F. James, P. Hobbs, J. Lockyear, S. V. Bulanov, and F. Pegoraro, Application of proton radiography in experiments of relevance to inertial confinement fusion, The European Physical Journal D 55 (2009), no. 2, 299-303.

[190] G Sarri, C a Cecchetti, L Romagnani, C M Brown, D J Hoarty, S James, J Morton, M E Dieckmann, R Jung, O Willi, S V Bulanov, F Pegoraro, and M Borghesi, The application of laser-driven proton beams to the radiography of intense laserhohlraum interactions, New Journal of Physics 12 (2010), no. 4, 045006 . 
[191] G. Sarri, W. Schumaker, A. Di Piazza, M. Vargas, B. Dromey, M. E. Dieckmann, V. Chvykov, A. Maksimchuk, V. Yanovsky, Z. H. He, B. X. Hou, J. a. Nees, a. G. R. Thomas, C. H. Keitel, M. Zepf, and K. Krushelnick, TableTop Laser-Based Source of Femtosecond, Collimated, Ultrarelativistic Positron Beams, Physical Review Letters 110 (2013), no. 25, 255002.

[192] T Schlegel, N Naumova, V T Tikhonchuk, C Labaune, I V Sokolov, and G Mourou, Relativistic laser piston model: Ponderomotive ion acceleration in dense plasmas using ultraintense laser pulses, Physics of Plasmas 16 (2009), no. 8, 083103.

[193] D. W. Schumacher, G. E. Kemp, A. Link, R. R. Freeman, and L. D. Van Woerkom, The shaped critical surface in high intensity laser plasma interactions, Physics of Plasmas 18 (2011), no. 1, 013102.

[194] Julian Schwinger, On Gauge Invariance and Vacuum Polarization, Physical Review 82 (1951), no. 5, 664-679.

[195] R H H Scott, C Beaucourt, H.-P. Schlenvoigt, K Markey, K L Lancaster, C P Ridgers, C M Brenner, J Pasley, R J Gray, I O Musgrave, A P L Robinson, K Li, M M Notley, J R Davies, S D Baton, J J Santos, J.-L. Feugeas, Ph. Nicolaï, G Malka, V T Tikhonchuk, P. McKenna, D Neely, S J Rose, and P A Norreys, Controlling Fast-Electron-Beam Divergence Using Two Laser Pulses, Physical Review Letters 109 (2012), no. 1, 015001.

[196] Y. Sentoku and A.J. Kemp, Numerical methods for particle simulations at extreme densities and temperatures: Weighted particles, relativistic collisions and reduced currents, Journal of Computational Physics 227 (2008), no. 14, 6846 6861. 
[197] F. H. Seguin, C. K. Li, M. J.-E. Manuel, H. G. Rinderknecht, N. Sinenian, J. a. Frenje, J. R. Rygg, D. G. Hicks, R. D. Petrasso, J. Delettrez, R. Betti, F. J. Marshall, and V. a. Smalyuk, Time evolution of filamentation and selfgenerated fields in the coronae of directly driven inertial-confinement fusion capsules, Physics of Plasmas 19 (2012), no. 1, 012701.

[198] A. Sgattoni, P. Londrillo, A. Macchi, and M. Passoni, Laser ion acceleration using a solid target coupled with a low-density layer, Physical Review E $\mathbf{8 5}$ (2012), no. 3, 1-9.

[199] L. O. Silva, Michael Marti, Jonathan R Davies, Ricardo A Fonseca, Chuang Ren, Frank S Tsung, and Warren B Mori, Proton Shock Acceleration in LaserPlasma Interactions, Physical Review Letters 1 (2004), no. JANUARY, 1-4.

[200] T. Sokollik, M. Schnurer, S. Ter-Avetisyan, S. Steinke, P. V. Nickles, W. Sandner, M. Amin, T. Toncian, O. Willi, a. a. Andreev, Paul R. Bolton, Hiroyuki Daido, and Sergei V. Bulanov, Proton Imaging Of Laser Irradiated Foils And Mass-Limited Targets, AIP Conference Proceedings 364 (2009), 364-373.

[201] I. Spencer, K.W.D. Ledingham, R.P. Singhal, T. McCanny, P. McKenna, E.L. Clark, K. Krushelnick, M. Zepf, F.N. Beg, M. Tatarakis, A.E. Dangor, P.a. Norreys, R.J. Clarke, R.M. Allott, and I.N. Ross, Laser generation of proton beams for the production of short-lived positron emitting radioisotopes, Nuclear Instruments and Methods in Physics Research Section B: Beam Interactions with Materials and Atoms 183 (2001), no. 3-4, 449-458.

[202] E. A. Startsev and C. J. McKinstrie, Multiple scale derivation of the relativistic ponderomotive force, Physical Review E 55 (1997), no. 6, 7527-7535. 
[203] D. Strickland and G. Mourou, Compression of Amplified Chirped Optical Pulses, Optics Communications 56 (1985), no. 3, 219-221.

[204] M. Tabak, James Hammer, Michael E Glinsky, W. L. Kruer, S. C. Wilks, John Woodworth, E Michael Campbell, Michael D Perry, and Rodney J Mason, Ignition and high gain with ultrapowerful lasers, Physics of Plasmas 1 (1994), no. May, 1626-1634.

[205] T. Tajima and J. Dawson, Laser Electron Accelerator, Physical Review Letters 43 (1979), no. 4, 267-270.

[206] A. H. Taub, Relativistic Rankine-Hugoniot Equations, Physical Review 74 (1948), no. 3, 1-7.

[207] W. Theobald, K. Akli, R. Clarke, J. a. Delettrez, R. R. Freeman, S. Glenzer, J. Green, G. Gregori, R. Heathcote, N. Izumi, J. a. King, J. a. Koch, J. Kuba, K. Lancaster, a. J. MacKinnon, M. Key, C. Mileham, J. Myatt, D. Neely, P. a. Norreys, H.-S. Park, J. Pasley, P. Patel, S. P. Regan, H. Sawada, R. Shepherd, R. Snavely, R. B. Stephens, C. Stoeckl, M. Storm, B. Zhang, and T. C. Sangster, Hot surface ionic line emission and cold K-inner shell emission from petawattlaser-irradiated Cu foil targets, Physics of Plasmas 13 (2006), no. 4, 043102.

[208] L Volpe, R Jafer, B Vauzour, Ph Nicolai, J J Santos, F Dorchies, C Fourment, S Hulin, C Regan, F Perez, S Baton, K Lancaster, M Galimberti, R Heathcote, M Tolley, Ch Spindloe, W Nazarov, P Koester, L Labate, L a Gizzi, C Benedetti, A Sgattoni, M Richetta, J Pasley, F N Beg, S Chawla, D P Higginson, A G MacPhee, and D Batani, Proton radiography of cylindrical laser-driven implosions, Plasma Physics and Controlled Fusion 53 (2011), no. 3, 032003. 
[209] E. Weibel, Weibel Instability, Physical Review Letters 2 (1959), no. 3.

[210] D.R. Welch and R.E. Clark, Advanced Particle-in-Cell Techniques for HEDP Simulation - LSP, (2011).

[211] D.R. Welch, D.V. Rose, R.E. Clark, T.C. Genoni, and T.P. Hughes, Implementation of an non-iterative implicit electromagnetic field solver for dense plasma simulation, Computer Physics Communications 164 (2004), no. 1-3, 183-188.

[212] S. C. Wilks, HED Seminar on Positrons (January), 2011.

[213] S. C. Wilks, J. M. Dawson, W. B. Mori, T. Katsouleas, and M. E. Jones, Photon Accelerator, Physical Review Letters (1989).

[214] S. C. Wilks and W. L. Kruer, Absorption of ultrashort, ultra-intense laser light by solids and overdense plasmas, IEEE Journal of Quantum Electronics 33 (1997), no. 11, 1954-1968.

[215] S. C. Wilks, W. L. Kruer, M. Tabak, and A. B. Langdon, Absorption of ultraintense laser pulses, Physical Review Letters 69 (1992), no. 9, 1383-1386.

[216] S. C. Wilks, A. B. Langdon, T. E. Cowan, M. Roth, M. Singh, S. Hatchett, M. H. Key, D. Pennington, A. MacKinnon, and R. a. Snavely, Energetic proton generation in ultra-intense lasersolid interactions, Physics of Plasmas 8 (2001), no. 2,542 .

[217] L. Willingale, P. M. Nilson, M. C. Kaluza, a. E. Dangor, R. G. Evans, P. Fernandes, M. G. Haines, C. Kamperidis, R. J. Kingham, C. P. Ridgers, M. Sherlock, a. G. R. Thomas, M. S. Wei, Z. Najmudin, K. Krushelnick, S. Bandyopadhyay, M. Notley, S. Minardi, M. Tatarakis, and W. Rozmus, Proton deflectometry of a magnetic reconnection geometry, Physics of Plasmas 17 (2010), no. 4, 043104. 
[218] L. Willingale, P. M. Nilson, a. G. R. Thomas, J. Cobble, R. S. Craxton, a. Maksimchuk, P. a. Norreys, T. C. Sangster, R. H. H. Scott, C. Stoeckl, C. Zulick, and K. Krushelnick, High-Power, Kilojoule Class Laser Channeling in MillimeterScale Underdense Plasma, Physical Review Letters 106 (2011), no. 10, 105002.

[219] L. Yin, B. J. Albright, B. M. Hegelich, K. J. Bowers, K. a. Flippo, T. J. T. Kwan, and J. C. Fernandez, Monoenergetic and GeV ion acceleration from the laser breakout afterburner using ultrathin targets, Physics of Plasmas 14 (2007), no. 5,056706 .

[220] Wei Yu, Lihua Cao, M.Y. Yu, A.L. Lei, Z.M. Sheng, H.B. Cai, K. Mima, and X.T. He, Focusing of intense laser pulse by a hollow cone, Laser and Particle Beams 28 (2010), no. 02, 293-298.

[221] T. Chiueh \& M. C. Begelman Z.-Y. Li, Electromagnetically driven relativistic jets - a class of self-similar solutions, Astrophys. J. 394 (1992), no. 459.

[222] Xiahui Zeng and Dianyuan Fan, Numerical simulations of laser light guiding and focusing using an open hollow cone, Optics Communications 283 (2010), no. $20,3854-3859$.

[223] Zongqing Zhao, Lihua Cao, Leifeng Cao, Jian Wang, Wenzhong Huang, Wei Jiang, Yingling He, Yuchi Wu, Bin Zhu, Kegong Dong, Yongkun Ding, Baohan Zhang, Yuqiu Gu, M. Y. Yu, and X. T. He, Acceleration and guiding of fast electrons by a nanobrush target, Physics of Plasmas 17 (2010), no. 12, 123108.

[224] A B Zylstra, C K Li, H G Rinderknecht, F H Séguin, R D Petrasso, C Stoeckl, D D Meyerhofer, P Nilson, T C Sangster, S Le Pape, A Mackinnon, and P Patel, Using high-intensity laser-generated energetic protons to radiograph di- 
rectly driven implosions., The Review of scientific instruments 83 (2012), no. 1, 013511. 